\title{
Computation and selection of equilibria in noncooperative games
}

Citation for published version (APA):

Peeters, R. J. A. P. (2002). Computation and selection of equilibria in noncooperative games. [Doctoral Thesis, Maastricht University]. Universiteit Maastricht. https://doi.org/10.26481/dis.20020621rp

Document status and date:

Published: 01/01/2002

DOI:

10.26481/dis.20020621rp

Document Version:

Publisher's PDF, also known as Version of record

\section{Please check the document version of this publication:}

- A submitted manuscript is the version of the article upon submission and before peer-review. There can be important differences between the submitted version and the official published version of record.

People interested in the research are advised to contact the author for the final version of the publication, or visit the DOI to the publisher's website.

- The final author version and the galley proof are versions of the publication after peer review.

- The final published version features the final layout of the paper including the volume, issue and page numbers.

Link to publication

\footnotetext{
General rights rights.

- You may freely distribute the URL identifying the publication in the public portal. please follow below link for the End User Agreement:

www.umlib.nl/taverne-license

Take down policy

If you believe that this document breaches copyright please contact us at:

repository@maastrichtuniversity.nl

providing details and we will investigate your claim.
}

Copyright and moral rights for the publications made accessible in the public portal are retained by the authors and/or other copyright owners and it is a condition of accessing publications that users recognise and abide by the legal requirements associated with these

- Users may download and print one copy of any publication from the public portal for the purpose of private study or research.

- You may not further distribute the material or use it for any profit-making activity or commercial gain

If the publication is distributed under the terms of Article $25 \mathrm{fa}$ of the Dutch Copyright Act, indicated by the "Taverne" license above, 


\title{
Computation and Selection of Equilibria in Noncooperative Games
}

\author{
Ronald Peeters
}

2002

Noncooperative game theory is a mathematical discipline that analyzes conflict situations. Often it serves as a tool for applied studies in which conflict situations arise. A frequently used solution concept in noncooperative game theory, that finds its origin in the description of rational behavior, is the equilibrium concept. However, equilibria are not easily computed, in particular when the game under study is large in size or when dynamics are involved. Furthermore, a noncooperative game may possess multiple equilibria. Both are reasons that might cause a hampering in the further use of game theory as a tool for applied studies. Therefore it is important to have fast algorithms to compute and select equilibria. This monograph is a contribution towards the development of such algorithms. 
This book was typeset by the author using $\mathrm{AT}_{\mathrm{E}} \mathrm{X}$.

\section{(c) 2002 Ronald Peeters}

All rights reserved. No part of this publication may be reproduced, stored in a retrieval system, or transmitted, in any form, or by any means, electronic, mechanical, photocopying, recording or otherwise, without the prior permission in writing from the author.

Published by University Press Maastricht

ISBN 90-5278-349-7

Printed in The Netherlands by Datawyse Maastricht 


\title{
Computation and Selection of Equilibria in Noncooperative Games
}

\author{
PROEFSCHRIFT
}

ter verkrijging van de graad van doctor aan de Universiteit Maastricht, op gezag van de Rector Magnificus,

Prof. Dr. A.C. Nieuwenhuijzen Kruseman, volgens het besluit van het College van Decanen, in het openbaar te verdedigen op vrijdag 21 juni 2002 om 14.00 uur

door

Ronald Peeters 


\section{Promotores:}

Prof. dr. P.J.J. Herings

Prof. dr. A.A. Stoorvogel (Technische UniversiteitEindhoven, Technische UniversiteitDelft)

\section{Beoordelingscommissie:}

Prof. dr. H.J.M. Peters (voorzitter)

Prof. dr. A.J.J. Talman (Katholieke Universiteit Brabant)

Dr. A.J. Vermeulen 
Many people have a passionate hatred of abstraction, chiefly, I think, because of its intellectual difficulty; but as they do not wish to give this reason, they invent all sorts of others that sound grand. They say that all reality is concrete, and that in making abstractions we are leaving out the essential. They say that all abstraction is falsification, and that as soon as you have left out any aspect of something actual you have exposed yourself to the risk of fallacy in arguing from its remaining aspects alone. Those who argue in this way are, in fact, concerned with matters quite other than those that concern science.

[Bertrand Russell (1931), The Scientific Outlook, page 85.]

The laws of science are written in this grand book, the universe, which stands continually open to our gaze. But the book cannot be understood unless one first learns to comprehend the language and read the letters in which it is composed. It is written in the language of mathematics and its characters are triangles, circles, and other geometric figures without which it is humanly impossible to understand a single word of it.

[Galileo Galilei (1623), Il Saggiatore (The Assayer).] 



\section{Acknowledgements}

This monograph is the result of research carried out over a period of four years. The first two years the research was financially supported by the SamenwerkingsOrgaan Brabantse Universiteiten, a cooperation body between Tilburg University and Eindhoven University of Technology. The other two years were supported by Universiteit Maastricht.

This work would never have been completed without the help of several people. First of all, my two promotores Jean-Jacques Herings and Anton Stoorvogel. Not only for the reason that their knowledge and expertise helped me grow, but also for the enthusiasm with which they supervised me. The best way I know how to show them my gratitude, is by quoting Sir Isaac Newton "If I have seen further ... it is by standing on the shoulders of giants."

Next, I would like to thank Hans Peters for chairing the promotion committee, and Dolf Talman and Dries Vermeulen for taking place in the promotion committee, the meticulous proofreading of the entire manuscript and the pleasant discussions I had with each one of them. Further, I want to thank Maarten Pieter Schinkel who was co-author of the last chapter of this monograph. Finally, a personal thanks goes to Jos Potters, who opened my eyes for doing research in game theory. At the end he is the guru to whom I owe having started to write this monograph.

More generally, I would like to thank all my old colleagues at the Department of Econometrics of Tilburg University and Department of Mathematics of Eindhoven University of Technology and my current colleagues at the Department of Economics of Universiteit Maastricht.

Last, but certainly not least, special thanks goes out to everyone who has supported me in one way or another. Some of them deserve to be named personally: my father, my mother, my brother Patrick, my sister Annuska, and my girlfriend Katja who is also thanked for her patience while postponing our lives together because of this dissertation.

RP, Beers, May 3, 2002 


\section{Contents}

Acknowledgements

Contents $\quad$ v

List of Figures $\quad x$

List of Tables $\quad$ xi

1 Introduction 1

2 Mathematical Preliminaries 9

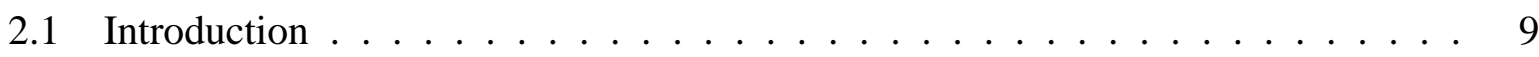

2.2 Some notation . . . . . . . . . . . . . . . . . . . . . 10

2.3 Relations, correspondences, and fixed points . . . . . . . . . . . . 11

2.4 Differential topology . . . . . . . . . . . . . . . . . . . 13

I Normal Form Games $\quad 19$

3 Computation of Equilibria $\quad 21$

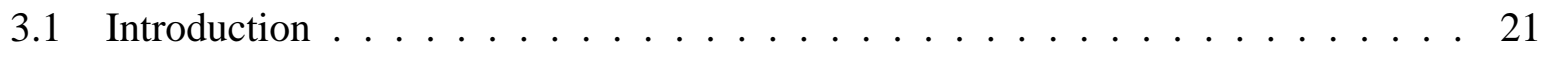

3.2 The linear tracing procedure $\ldots \ldots \ldots \ldots \ldots \ldots \ldots \ldots$

3.3 A piecewise differentiable approach $\ldots \ldots \ldots \ldots \ldots . \ldots 25$

3.4 A differentiable approach . . . . . . . . . . . . . . . . 32

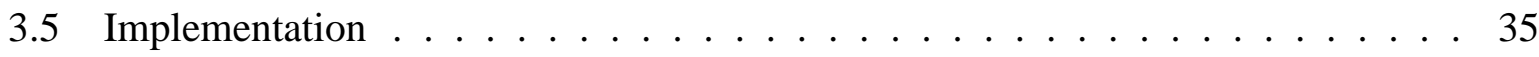

3.6 Summary $\ldots \ldots \ldots \ldots \ldots$

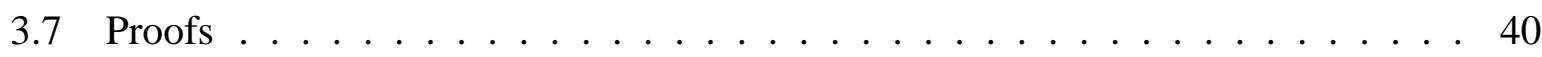

4 Computation of All Equilibria $\quad 49$

4.1 Introduction . . . . . . . . . . . . . . . . . . . . . . . . . 49

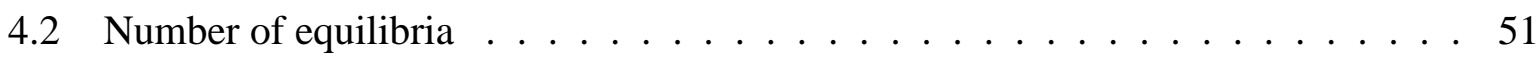


4.3 Equilibria as solutions to systems of equations . . . . . . . . . . . . 52

4.4 Implementation . . . . . . . . . . . . . . . . 56

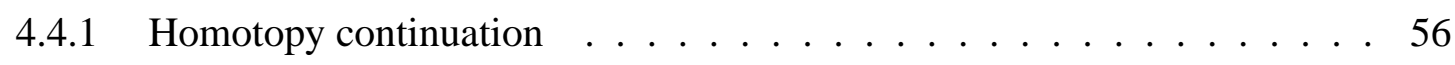

4.4 Hompack .......................... 57

4.4.3 Applying the Gröbner basis . . . . . . . . . . . . . . . . 59

4.5 Description of algorithm . . . . . . . . . . . . . . . 60

4.6 An alternative method . . . . . . . . . . . . . . . 61

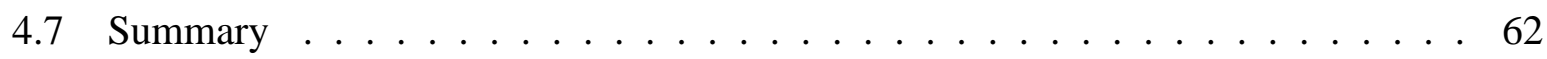

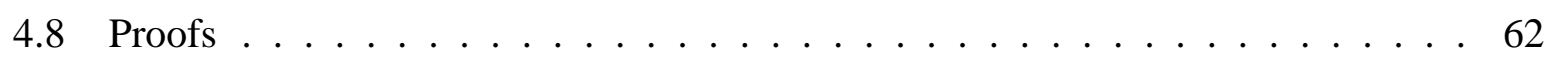

II Stochastic Games $\quad 65$

5 Equilibrium Selection $\quad 67$

5.1 Introduction . . . . . . . . . . . . . . . 67

5.2 Finite discounted stochastic games . . . . . . . . . . . . . 68

5.3 Linear tracing procedure . . . . . . . . . . . . . . 72

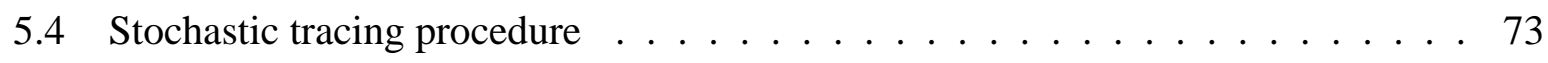

5.4 .1 Alternative 1: $\mathrm{C}(\mathrm{S}), \mathrm{I}(\mathrm{T}) \ldots \ldots \ldots \ldots . \ldots 74$

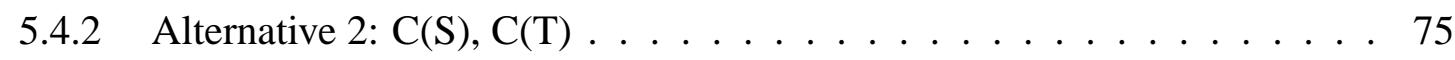

5.4 .3 Alternative $3: \mathrm{I}(\mathrm{S}), \mathrm{I}(\mathrm{T}) \ldots \ldots \ldots \ldots \ldots \ldots$

5.4 .4 Alternative $4: \mathrm{I}(\mathrm{S}), \mathrm{C}(\mathrm{T}) \ldots \ldots \ldots \ldots \ldots$

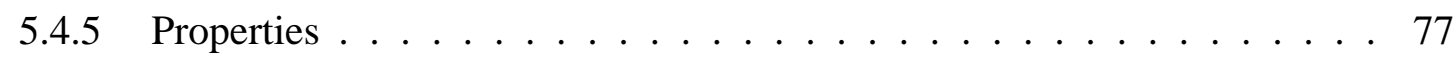

5.5 Analysis of alternatives . . . . . . . . . . . . . . . . 77

5.5 .1 Beliefs within states . . . . . . . . . . . . 77

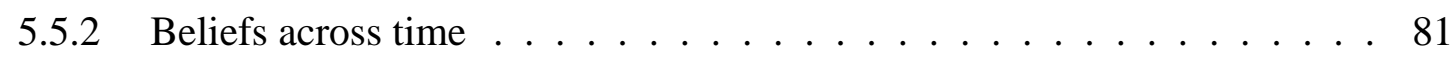

5.5.3 Extending the linear tracing procedure . . . . . . . . . . 85

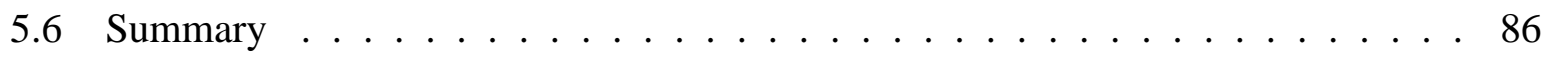

6 Computation of Stationary Equilibria $\quad 87$

6.1 Introduction . . . . . . . . . . . . . . . 87

6.2 Characterization of stationary equilibria . . . . . . . . . . . . 89

6.3 The stochastic tracing procedure ....................... 93

6.4 Structure of the stochastic tracing procedure . . . . . . . . . . . . 95

6.5 Smoothing the stochastic tracing procedure . . . . . . . . . . 100

6.6 Implementation . . . . . . . . . . . . . . . . . 102

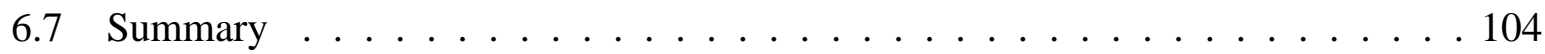

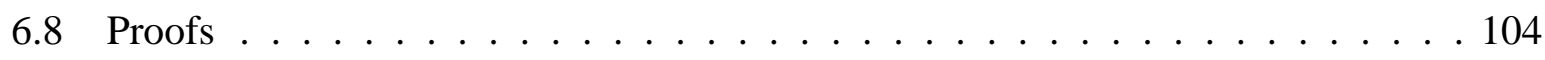




\section{Application}

7 Dynamic Competition $\quad 109$

7.1 Introduction . . . . . . . . . . . . . . . . . . 109

7.2 The dynamic entry-and-exit model . . . . . . . . . . . . . . 112

7.3 Finite stochastic game model . . . . . . . . . . . . . . . . 113

7.4 When there are two firms . . . . . . . . . . . . . . 116

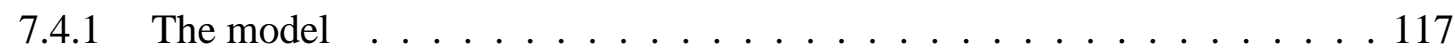

7.4.2 Alternating monopoly . . . . . . . . . . . . . 117

7.4.3 Speed of convergence to and robustness of alternating monopoly . . . . . 119

7.5 Concluding thoughts . . . . . . . . . . . . . . . . 121

$\begin{array}{lr}\text { References } & 123\end{array}$

$\begin{array}{ll}\text { Samenvatting } & 133\end{array}$

$\begin{array}{ll}\text { Curriculum Vitae } & 137\end{array}$ 


\section{List of Figures}

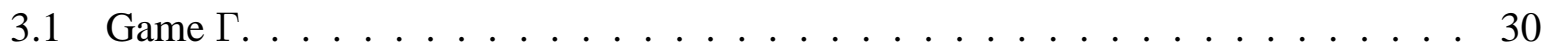

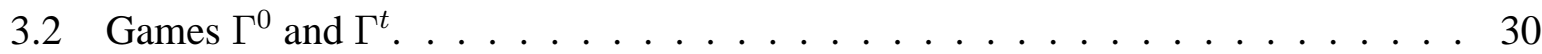

3.3 The linear tracing procedure. . . . . . . . . . . . . . . . 31

3.4 Relation between $\sigma_{j}^{i}$ and $\alpha_{j}^{i}$ and between $\lambda_{j}^{i}$ and $\alpha_{j}^{i} \ldots \ldots \ldots \ldots \ldots \ldots$

3.5 Homotopy-variables of the linear tracing procedure as a function of pathlength $\tau$. 35

3.6 The behavior of the homotopy-variables along the zero curve as a function of

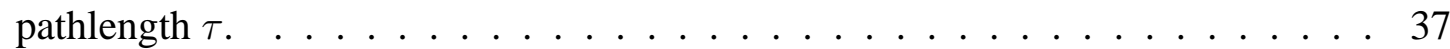

3.7 The matrix of derivatives of $g^{B^{*}}$ and $h^{B^{*}} \ldots \ldots \ldots \ldots \ldots \ldots \ldots \ldots$

3.8 The Jacobian of the homotopy function $\mathcal{H} \ldots \ldots \ldots$. . . . . . . . . . . 45

3.9 Decomposition of the Jacobian of $\mathcal{H}$ into two matrices. . . . . . . . . . . . 46

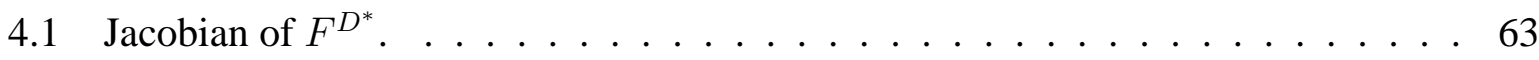

$5.1 \quad$ Normal form game $\Gamma \ldots \ldots \ldots \ldots$. . . . . . . . . . . . . . . 78

5.2 Game $\Gamma^{t}$ when $\mathrm{I}(\mathrm{S})$ is assumed. . . . . . . . . . . . . . . . . . . . . . . . 79

5.3 Feasible path when $\mathrm{I}(\mathrm{S})$ is assumed. . . . . . . . . . . . . . . 80

5.4 Game $\Gamma^{t}$ when $\mathrm{C}(\mathrm{S})$ is assumed. . . . . . . . . . . . . . . . . 80

5.5 Feasible path when $\mathrm{C}(\mathrm{S})$ is assumed. . . . . . . . . . . . . . 81

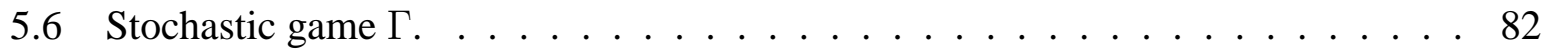

5.7 Game $\Gamma^{0}$ when $\mathrm{I}(\mathrm{T})$ is assumed. . . . . . . . . . . . . . . . . . 84

5.8 Feasible path when $\mathrm{I}(\mathrm{T})$ is assumed. $\ldots \ldots \ldots \ldots$

5.9 Feasible path when $\mathrm{C}(\mathrm{T})$ is assumed. . . . . . . . . . . . . . . . . 85

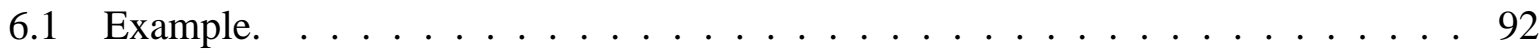

6.2 The structure of $\mathcal{S}(\Gamma, p) \ldots \ldots \ldots \ldots \ldots$

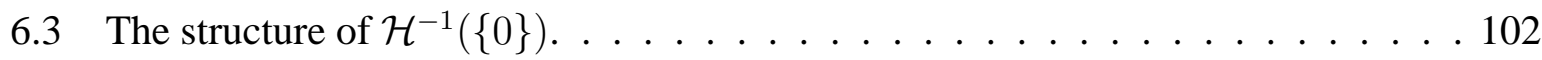

6.4 The matrix of partial derivatives of $g^{B^{*}}$ and $h^{B^{*}} \ldots \ldots \ldots \ldots \ldots \ldots$

7.1 The dynamic entry-and-exit model as a stochastic game. . . . . . . . . . 117

7.2 Transition-probabilities in market structure. . . . . . . . . . . . . . . 120

7.3 Speed of convergence to alternating monopoly for different discount factors. . . . 121 


\section{List of Tables}

3.1 Description of the sets $\mathcal{L}\left(\Gamma, p, B^{*}\right) \ldots \ldots \ldots \ldots$

3.2 Average computation times and average number of function evaluations. . . . . . 38

3.3 Average number of equilibria. . . . . . . . . . . . . . . . 38

3.4 Multiplicity by variation of prior. . . . . . . . . . . . . . . . 39

4.1 Mean number of equilibria. . . . . . . . . . . . . . . . 51

4.2 Total number of solutions. . . . . . . . . . . . . . . . . . . 55

6.1 Average computation times in seconds. . . . . . . . . . . . 103

7.1 Stationary Cournot equilibrium with two active firms. . . . . . . . . . 118

7.2 Stationary equilibrium with alternating monopoly. . . . . . . . . . . 118 


\section{Chapter 1}

\section{Introduction}

The foundation of Game Theory was laid in an article by John von Neumann (1928), starting with the following sentence:

"Die Frage, deren Beantwortung die vorliegende Arbeit anstrebt, ist die folgende:

n Spieler, $S_{1}, S_{2}, \ldots, S_{n}$, spielen ein gegebnes Gesellschaftsspiel $\mathcal{G}$. Wie muß einer dieser Spieler, $S_{m}$, spielen, um dabei ein möglichst günstiges Resultat zu erzielen?"

The theory received widespread attention only after publication of the fundamental book of von Neumann and Morgenstern (1944, p.31), where the aim of Game Theory is described as follows:

"[W]e wish to find the mathematically complete principles which define "rational behavior" for the participants in a social economy, and to derive from them the general characteristics of that behavior. And while the principles ought to be perfectly general - i.e., valid in all situations - we may be satisfied if we can find solutions, for the moment, only in some characteristic special cases."

Informally, Game Theory is a mathematical discipline that analyzes conflict situations. A conflict situation - the game - is a situation in which a certain number of individuals - the players interact and thereby jointly determine the outcome. Each participating player has partial control over the situation, but never full control. Each player is endowed with certain individual preferences over the set of possible outcomes and strives to obtain the outcome that is most profitable to him. Under some minor assumptions these preferences can be represented by a von NeumannMorgenstern utility function that characterizes the preferences by a real valued function whose expected value the player strives to maximize. The aim of Game Theory is to prescribe which strategy each player in a game should play such that his partial influence on the situation is exploited in order to promote his interest optimally. Hence, the aim of Game Theory is to provide a solution - to give a characterization of rational behavior - for every game. 
Traditionally, games have been divided into two classes: cooperative games and noncooperative games. In this monograph, only noncooperative games are dealt with. A noncooperative game is a game in which there are no possibilities for communication, correlation or (pre)commitment, except for those that are explicitly captured by the rules of the game. A solution of such a game is a set of recommendations, that tells each player how to behave in every situation that may arise. This solution should be consistent, i.e. no player should have an incentive to deviate from his recommendation. Hence, a solution must be self-enforcing: for each player it should not be in his interest to deviate as long as his opponent players obey their recommendations. In game theoretic terminology this means that the solution should be a Nash equilibrium (Nash $(1950,1951)$ ), i.e. a strategy combination - consisting of one strategy for each player - with the property that no player can gain (in terms of utility) by unilaterally deviating from it.

Many research areas in which conflicts between agents arise - as in economics and other social sciences - benefit from the presence of game theoretic tools. Many papers in such areas have the following structure: a problem is modeled as a game, the game is analyzed by computing its equilibria, and the properties of the latter are translated back into insights relevant to the original problem (see van Damme (1995)). However, solving for Nash equilibria can be a tedious, error-prone affair. Even when the game that is object of study is very simple. And even if an equilibrium is found, it may not be the case that the equilibrium has a sound game theoretic underpinning, since, in general, a game possesses multiple Nash equilibria. Consequently, having numerical methods to compute and to select on equilibria might cause further progress in research programs where game models arise.

The appropriate method for computing Nash equilibria for a game depends on a number of factors. The first and most important factor involves whether one wants to obtain simply one sample equilibrium or all equilibria.

The second factor of importance concerns whether, or not, the number of players is greater than two. For two person games there exist methods for finding exact sample Nash equilibria. For games with more than two players, however, this is in general not possible anymore. The methods that work for two person games can typically not be directly extended for games with more than two players. This factor was already recognized in von Neumann (1928):

“[D]as 3-Personen-Spiel ist etwas wesentlich anderes als das von zwei Personen.”

The third factor that determines the choice of method concerns the type of Nash equilibrium one wishes to find. It is well-known that not all Nash equilibria are equally attractive. Therefore refinement criteria for selecting among multiple equilibria are proposed. There exists a large literature on equilibrium refinements. For a condensed writing on the issue of equilibrium selection, see van Damme (1987). 
For most methods that find a sample equilibrium, it is not guaranteed that the equilibrium found will satisfy a given refinement condition or selection criterion. So, any method intended to find a sample Nash equilibrium that satisfies particular refinement conditions needs to be modified. Since the set of equilibria satisfying particular refinement conditions is a subset of the set of all Nash equilibria, a method that finds all Nash equilibria can serve as a basis for a method to find the set of all refined Nash equilibria - as long as the set of refined Nash equilibria can be characterized as a subset of the whole set of Nash equilibria in a computable way.

Noncooperative game theory is divided in two parts: static games and dynamic games. A static game is a game that takes place in one moment of time: all players make their choice once and simultaneous and, dependent on the choices made, each player obtains its payoff. In a dynamic game, strategic decisions have to be made during several stages. Crucial in such games is the information available to a player about choices made prior to the occasion, the duration of the game, and the way outcomes are evaluated in terms of payoff.

A successful application of game theory to problems in which conflict situations arise, is often hindered by the fact that the dynamics complicates the problems rapidly with regard to computational purposes. Even in absence of dynamics, problems become complicated rapidly when the number of players, or the number of actions available to the players grows. A solution to this is found in the development of new algorithms that are tailor-made to specific problems and that are easily implementable. Moreover, such algorithms should compute equilibria with a sound game theoretic underpinning, in the common case that a game possesses multiple equilibria. See also Judd (1997, 1998) and McKelvey and McLennan (1996) for an expression of the important role of computational methods in the further development of economic theory.

In Chapter 2 the mathematical preliminaries are given. The chapter contains an overview of some mathematical notions used later on. The exposition is not complete as the reader is assumed to have a basic knowledge of set theory, topology, vector spaces, matrix theory, measure theory, probability theory, stochastic processes, complex numbers, and differential calculus. The goal of Chapter 2 is to make the monograph as accessible as possible. Of special importance for the developments of this monograph is the section dealing with differential topology.

Part I of this monograph is devoted to games in normal form. In these games there are a finite number of players which all have a finite number of actions at their disposal to influence the outcome of the game. Given the simultaneously and independently chosen actions, an outcome results which immediately determines the payoff to each one of the players. Assumed is that players may randomize their actions, i.e. they decide on probability distributions on their action sets. Payoffs are then equal to the expected value of the payoffs. Each player is assumed to maximize its expected payoff. A Nash equilibrium for such a game is a tuple of strategies consisting of one strategy for each player - where each player's strategy is an optimal strategy against the other players' strategies. 
The first method to compute a Nash equilibrium for a normal form game is the one developed by Lemke and Howson (1964). Their algorithm, which belongs to the class of path following algorithms, provides a way to find at least one Nash equilibrium for any two person game. It solves bimatrix games - normal form games in which two players are involved - by solving a linear complementarity problem. Although multiple Nash equilibria can be found, there is no guarantee that all Nash equilibria are found. As long as the data of the problem are rational, the algorithm can provide exact solutions, since all computations are done in the rational field.

Rosenmüller (1971) and Wilson (1971) independently and simultaneously extended the algorithm of Lemke and Howson to find Nash equilibria for $n$-person games. They both discovered that $n$-person games can be formulated as a non-linear complementarity problem. Based on features of these nonconstructive methods, Garcia, Lemke and Lüthi (1973) were the first to propose a simplicial algorithm. Their method was derived from the work of Scarf $(1967,1973)$ for computing a Nash equilibrium of $n$-person games thereby launching the first implementable algorithm to solve such games. Later, a more efficient simplicial algorithm was proposed in van der Laan and Talman (1982).

The literature mentioned above only covered the issue of computation but did not concern the issue of selection. For bimatrix games, the modification of the Lemke-Howson algorithm by Eaves (1971) causes that the resulting algorithm will only terminate at a perfect equilibrium, a refinement introduced in Selten (1975). A second procedure to search for a perfect equilibrium of a bimatrix game was developed by van den Elzen and Talman (1991). For the same class of games Wilson (1992) presented an algorithm to compute simply stable equilibria. For the class of $n$ person games, Yamamoto (1993) describes a path-following procedure for the computation of a proper equilibrium, a refinement introduced in Myerson (1978). This procedure is not directly fit for implementation though. Talman and Yang (1998) describe an algorithm for the computation of a proper equilibrium that is implementable.

For bimatrix games, the algorithm of van den Elzen and Talman (1999) computes the Nash equilibrium selected by the 'linear tracing procedure' (see Harsanyi and Selten (1988)). The generalization to $n$-person games is covered by the simplicial method presented in Herings and van den Elzen (2002). Simplicial methods solve a non-linear equilibrium problem by solving a piecewise linear approximation of the problem. With the exception of the algorithm presented in this monograph, all known algorithms that have been shown to converge to a Nash equilibrium for a generic $n$-person game, are simplicial methods. A potential drawback of these methods is that they do not exploit the differentiable structure that is present in $n$-person games.

For an $n$-person game, an algorithm that exploits its differentiable structure is developed in Chapter 3. Besides just computing a sample equilibrium, it partly solves the problem of multiplicity by applying the linear tracing procedure. The linear tracing procedure has been introduced by Harsanyi (1975) and is used extensively in the selection theory proposed by Harsanyi and Sel- 
ten (1988). It concerns a mathematical construct that transforms subjective a priori expectations into equilibrium expectations. It is proved that the linear tracing procedure is well-defined for almost all normal form games. This means that for almost all normal form games the algorithm is able to compute - or, converges to - an equilibrium.

Next, in Chapter 4, a method to numerically solve for all Nash equilibria for the same class of games is described. Again, the method is based on an adjustment process that connects trivial solutions to equilibrium solutions via paths. The computation of all equilibria seems practically infeasible since the number of equilibria grows exponentially in the size of the game - given by the number of players and the number of strategies each player has at its disposal. As a partial resolution to this problem, the algorithm is developed such that during the course of the algorithm more and more equilibria are found. A similar point has been made to use branch-andbound methods, as opposed to the present heuristic methods, to solve problems of the travelingsalesman type and applying the up-to-date best solutions during the course of the computations (see Sierksma (2001)). Moreover, it is possible to improve on computing time considerably by applying parallel computing.

Dynamic games of finite duration, where players obtain their payoffs after all decisions are made and the game has terminated, are called games in extensive form. It is possible to apply algorithms to solve for normal form games for this class of games. This is possible in two manners. First, the game in extensive form can be reformulated as a game in agent normal form. This is achieved by treating the players for each information set at which a player has to act as a separate agent of this player. All agents corresponding to the same player obtain the payoff of that player in the original extensive form game. The resulting game can be solved as a normal form game. The second method is to start solving the smallest subgames and subsequently solving all subgames backwards until the whole game is solved. For this method the algorithm to solve normal form games has to be used successively.

In Harsanyi and Selten (1988), the tracing procedure is defined for normal form games and for extensive form games with a perfect recall information structure. For extensive form games, Harsanyi and Selten (1988) first transform the game into one in standard form and subsequently define the tracing procedure for that class of games. Computation of the Nash equilibrium selected by the tracing procedure in extensive form games is the topic of von Stengel, van den Elzen, and Talman (2001), who invoke the sequence form to calculate such equilibria efficiently.

Part II of this monograph is devoted to stochastic games. More precisely, finite discounted stochastic games with infinite time horizon are being studied. Such a stochastic game starts in an initial state, some element of a finite set of states. Given the initial state, all players simultaneously and independently have to make a strategic decision. Dependent on the strategic decisions made, each player obtains a payoff. Dependent on the strategic decisions and possibly on chance the state of the subsequent period is determined. In this subsequent period, players again have to 
make a strategic decision in order to partially influence the payoffs and the flow to the very next state - the game dynamics. This procedure continues ad infinitum.

In order to keep such problems with infinite horizon analyzable, chance is probabilistically conditioned on the present state and the chosen strategies and not on time. This has as direct result that strategies can be chosen time independent, or stationary. Focal point in Part II will be stationary equilibria, i.e. equilibria in which players use strategies that are state dependent but time independent. Several motivations for analyzing stationary equilibria can be found in Maskin and Tirole (2001). Stationary strategies prescribe the simplest form of behavior that is consistent with rationality, stationarity captures the notion that 'bygones are bygones' more completely than does the concept of subgame-perfect equilibrium, and it embodies the principle that 'minor causes should have minor effects', that is, only those aspects of the past that are 'significant' should have an appreciable influence on behavior. The pragmatic motivations they give are that in applied theory, the focus on stationary strategies allows for clean, unobstructed analysis of the influence of the state variables. Moreover, stationary strategies substantially reduce the number of parameters to be estimated in dynamic (econometric) models and stationary models can be simulated.

For most normal form games there is a vast multiplicity of equilibria, see McLennan (1999). There is no reason to expect that the situation is different for the multiplicity of stationary equilibria in stochastic games. For this reason, it is essential to provide an algorithm that selects a particular stationary equilibrium. Besides selecting on stationary equilibria, selection is further restricted by extending the linear tracing procedure in a natural way to stochastic games. Since the expected utility property is not satisfied in stochastic games, it is not possible to transform a stochastic game into one in standard form. The way to extend the tracing procedure to the class of stochastic games is neither straightforward nor unique. In Chapter 5 four natural extensions of the linear tracing procedure to the class of stochastic games are presented. It is shown that the four extensions are essentially different, and one of the four extensions is proposed as the most natural extension. This natural extension of the linear tracing procedure, the stochastic tracing procedure, transforms subjective a priori stationary expectations into stationary equilibrium expectations.

Stochastic games were introduced by Shapley (1953). He considered both finite and infinite horizon two-person zero-sum stochastic games with finite state space and finite action spaces. Shapley proved that such games have a value and that both players possess optimal stationary strategies with respect to the discounted payoff criterion.

Fink (1964), Takahashi (1964), and Sobel (1971) extended Shapley's model to general $n$ person stochastic games. For the model with finite state space and finite action spaces they showed the existence of a stationary equilibrium. In Breton, Filar, Haurie, and Schultz (1986), Schultz (1986), Filar, Schultz, Thuijsman, and Vrieze (1991), and Breton (1991) the problem 
of finding discounted stationary equilibria in the general $n$-person stochastic game is reduced to that of finding a global minimum in a nonlinear program with linear constraints. Solving this nonlinear program is equivalent to solving a certain nonlinear system for which it is known that the objective value in the global minimum is zero. But, as is noted in Breton (1991), the convergence of an optimization algorithm to the global optimum is not guaranteed.

In Chapter 6, it is proved that the stochastic tracing procedure as defined in Chapter 5 is well-defined for almost all stochastic games. This means that for almost all stochastic games there exists a unique path connecting the best responses of each player to subjective a priori stationary expectations about its opponents to a stationary equilibrium of the stochastic game. What remains is to determine the starting point and from there to follow numerically the path up to the stationary equilibrium. It is shown that the algorithm is globally convergent. This algorithm is the first globally convergent algorithm to solve for an equilibrium in an arbitrary $n$-person stochastic game. As another by-product of the convergence proof, an extension of a recent result of Haller and Lagunoff (2000) is found. Their main result is that the set of stationary equilibria in a stochastic game is generically finite. A corollary to the main result of Chapter 6 is that this finite number of equilibria is odd.

The algorithms developed for normal form games in Chapter 3 and Chapter 4 and the algorithm developed for stochastic games developed in Chapter 6 have in common that they are based on following paths from a trivial solution to a solution of the original problem. In Chapter 4 the paths are smooth by definition, whereas in Chapter 3 and Chapter 6 the paths became smooth after applying a transformation of variables as in Garcia and Zangwill (1981). In all these chapters, homotopy functions are described such that the paths correspond to a subset of the set of zeros of the homotopy function. To implement the algorithm, the only thing that is needed is having a numerical method to trace the homotopy-path after the starting point has been determined. There are several methods available to numerically trace homotopy-paths: predictor-corrector methods, and simplicial methods. Predictor-corrector methods approximately follow 'exact' solution curves, whereas simplicial methods follow approximate solution curves exactly. For more theory on path-tracking methods the reader is referred to Allgower and Georg $(1990,1993,1997)$, Garcia and Zangwill (1981), and Eaves and Schmedders (1999).

In this monograph, all implementations of the algorithms are based on a path-following method that is professionally programmed in FORTRAN. It concerns the software-package HoMPACK for which Watson, Billups, and Morgan (1987) is the condensed reference. As the proof of the pudding is in eating, this monograph ends with an application in Part III. More precisely, an application of stochastic games to an environment where a finite number of (potential) firms compete dynamically in the market for some homogeneous product is considered. In a given period, each firm is either an incumbent or a potential entrant. In each period, incumbents make a decision about the quantity to produce in that period and about whether to leave the market or 
to stay. Potential entrants only have to decide whether to enter or not in a given period.

Since all firms are assumed to have identical cost structures, the market structure (the state) in a certain period is determined by the number of firms that are active in production in that period. The instantaneous profits to an incumbent firm depend on the current market structure, the firm's own production quantity and its competitors' production quantities. When a potential entrant decides to enter, it has to make some sunk costs, which can be avoided by not entering. Tomorrow's market structure depends on the number of active firms today and the entry and exit decisions made by the firms.

In such dynamic entry-and-exit models, common understanding is that potential entrants will enter into the market up to the point where all excess profits are eroded. Dominant incumbent positions are possible only under specific circumstances, such as the presence of substantial barriers to entry, or when incumbents can credibly threaten to punish rivals with losses upon entry.

In Chapter 7, the case where there are two firms, no fixed costs and no entrance costs, is studied in more detail. Demand functions are assumed to be linear. The market is sufficiently big to admit two firms, i.e. two firms involved in Cournot competition that are both able to make positive profits. One would expect - as common understanding suggests - that in such an economic environment the market will be served by both firms in all periods. This repeated Cournot equilibrium is a stationary equilibrium indeed. However, by applying the numerical method described in Chapter 6 it is found that, when the discount factor is large enough, there is also an equilibrium inducing an alternating monopoly. At even periods one firm serves the market and at odd periods the other. The alternating monopoly equilibrium allows firms to take bi-periodically the full monopoly profit. The alternating monopoly equilibrium induces temporal market segregation, the temporal counterpart of the more frequently studied regional market segregation.

It can be shown that equilibrium profits are higher in the alternating monopoly equilibrium than in the repeated Cournot equilibrium, implying that firms have strong incentives to coordinate on the alternating monopoly equilibrium.

In a situation where both firms have already entered the market, coordination on the alternating monopoly equilibrium is still possible. In the state where both firms are present, each one will leave the market next period with a small probability. As soon as one firm has left, the alternating monopoly gets into action. Chapter 7 highlights the potential of numerical work and in particular how it may lead to new economic insights. 


\section{Chapter 2}

\section{Mathematical Preliminaries}

\subsection{Introduction}

In this chapter some mathematical concepts and results used in this monograph are presented. As the exposition given here does not yield a complete overview of the mathematical notions used later on, the reader is assumed to have a basic knowledge from set theory, topology, vector spaces, matrix theory, measure theory, probability theory, stochastic processes, complex numbers, and differential calculus. For a (more) complete exposition on the concepts presented in this chapter, the reader is referred to Chapter 2 of Herings (1996).

Section 2.2 starts with introducing some notation being used in this monograph, and in particular, in this chapter.

In Section 2.3 the concept of a relation is presented. In particular, a special type of relation, called correspondence, is introduced. Definitions, characterizations, and results concerning several forms of continuity of correspondences are given. Further, two fixed point theorems are formulated. The importance of fixed point theorems is the use of them in existence proofs. For instance, the existence of a Nash equilibrium can be easily proved by applying Kakutani's fixed point theorem. Browder's fixed point theorem can be used, for instance, for proving the feasibility of the linear tracing procedure.

Section 2.4 concludes this chapter with some notions from differential calculus and differential topology. The definitions of a manifold, a manifold with generalized boundary, and a regular constraint set, and some results like the inverse function theorem and the transversality theorem are given. These concepts are very useful to describe the structure of the linear tracing procedure in Chapter 3 and the structure of the stochastic tracing procedure in Chapter 6.

All concepts and results mentioned in this chapter are fairly well-known, and therefore no proofs are given. However, references are provided for all results. The presentation is minimal in the sense that no results are given that are not explicitly needed. 


\subsection{Some notation}

The set of natural numbers is denoted by $\mathbb{N}=\{1,2, \ldots\}, \mathbb{Z}=\{\ldots,-1,0,1, \ldots\}$ denotes the set of integers, $\mathbb{Z}_{+}=\{0,1, \ldots\}$ denotes the set of non-negative integers, $\mathbb{R}$ denotes the set of real numbers, $\mathbb{R}_{+}$denotes the set of non-negative real numbers and $\mathbb{C}$ denotes the set of complex numbers. For $m \in \mathbb{Z}_{+}$, the set of integers $\{1, \ldots, m\}$ is denoted by $I_{m}$ and the set of integers $\{0, \ldots, m\}$ is denoted by $I_{m}^{0}$. The set $\mathbb{N}^{*}$, defined by $\mathbb{N}^{*}=\mathbb{N} \cup\{+\infty\}$, is called the set of extended natural numbers.

Let some $m \in \mathbb{N}$ be given. The $m$-dimensional Euclidean space is denoted by $\mathbb{R}^{m}=X_{i=1}^{m} \mathbb{R}$ and is defined as the $m$-fold Cartesian product of $\mathbb{R}$. Finally, $\mathbb{C}^{m}=X_{i=1}^{m} \mathbb{C}$ denotes the $m$-fold Cartesian product of $\mathbb{C}$.

Let $X$ and $Y$ be two sets. If with every element $x$ of $X$ is associated exactly one element $y$ of $Y$, then a function $f$ from $X$ into $Y$ is defined, denoted by $f: X \rightarrow Y$. Let a function $f: X \rightarrow Y$ be given. The set $X$ is called the domain of $f$. If $x$ is an element of $X$, then $f(x)$ denotes the element $y$ of $Y$ associated with $x$ and is called the image by $f$ of $x$. Let $S$ be a subset of $X$ and let $T$ be a subset of $Y$. The image of $S$ by $f$, denoted by $f(S)$, is defined by $f(S)=\{y \in Y \mid \exists x \in S, y=f(x)\}$ and the inverse image of $T$ by $f$, denoted by $f^{-1}(T)$, is defined by $f^{-1}(T)=\{x \in X \mid f(x) \in T\}$. The image of $f$ by $X$ is called the range of $f$. The function $g: S \rightarrow Y$, defined by $g(x)=f(x)$, for all $x \in S$, is denoted by $f_{\mid S}$, and is called the restriction of $f$ to $S$. If $\bar{X}$ is a set containing $X$, and $g: \bar{X} \rightarrow Y$ is a function satisfying $g(x)=f(x)$, for all $x \in X$, then $g$ is called an extension of $f$ to $\bar{X}$. The function $f$ is said to be injective if $f^{-1}(\{y\})$ contains at most one element for every element $y$ of $Y$, and $f$ is said to be surjective if $f(X)=Y$. If the function $f$ is injective and surjective, then the function which associates every element $y$ of $Y$ the element $x$ of $X$ satisfying $f(x)=y$ is called the inverse of $f$ and is denoted by $f^{-1}: Y \rightarrow X$. The function $f$ is called a homeomorphism if it is continuous, injective, surjective, and has a continuous inverse. The sets $X$ and $Y$ are called homeomorphic if there exists a homeomorphism $f: X \rightarrow Y$.

Let $X$ be a topological space. The topological space $X$ is called an $\operatorname{arc}$ if $X$ and the closed unit interval $[0,1]$ are homeomorphic, $X$ is called a loop if $X$ and the unit circle in $\mathbb{R}^{2}$, being the set $\left\{x \in \mathbb{R}^{2} \mid\|x\|_{2}=1\right\}$, are homeomorphic. If $X$ is an arc and $f:[0,1] \rightarrow X$ is a homeomorphism, then $f(\{0,1\})$ is called the relative boundary of $X, f(0)$ and $f(1)$ are called the boundary points of $X$, and $X \backslash f(\{0,1\})$ is called the relative interior of $X$. It can be shown that both the relative boundary and the relative interior of $X$ are independent of the homeomorphism chosen. A continuous function $f:[0,1] \rightarrow X$ is called a path. The elements $f(0)$ and $f(1)$ are called the beginning and end points of the path $f$, respectively, and $f(0)$ and $f(1)$ are said to be joined by the path $f$.

A set $X$ is said to be finite if the number of its elements is a non-negative integer. A set $X$ is 
said to be countable if there exists an injective function $f$ from $X$ into a subset of $\mathbb{N}$. If $X$ is a finite set of elements, then $|X|$ denotes the cardinality of $X$, i.e. the number of elements of $X$.

Let a set $X$ be given. A function $f: \mathbb{N} \rightarrow X$ is called a sequence in $X$ and is denoted by $\left(x^{n}\right)_{n \in \mathbb{N}}$, where $x^{n}=f(n)$, for all $n \in \mathbb{N}$. If $\left(x^{n}\right)_{n \in \mathbb{N}}$ is a sequence in $X$ and $\left(n^{m}\right)_{m \in \mathbb{N}}$ is a sequence in $\mathbb{N}$ such that $n^{m}<n^{m+1}$, for all $m \in \mathbb{N}$, then $\left(x^{n^{m}}\right)_{m \in \mathbb{N}}$ is called a subsequence of $\left(x^{n}\right)_{n \in \mathbb{N}}$.

\subsection{Relations, correspondences, and fixed points}

Let $X$ and $Y$ be two sets. A relation $\varphi$ from $X$ into $Y$ associates with every element $x$ of $X$ a subset $\varphi(x)$ of $Y$, and is denoted by $\varphi: X \rightarrow Y$. The inverse image by $\varphi$ of $T$, denoted by $\varphi^{-1}(T)$, is defined by $\varphi^{-1}(T)=\{x \in X \mid \varphi(x) \cap T \neq \emptyset\}$. The set $\{(x, y) \in X \times Y \mid y \in \varphi(x)\}$ is called the graph of $\varphi$. If $Y$ is a topological space and $\varphi(x)$ is compact for every $x \in X$, then the relation $\varphi$ is said to be compact-valued. If $Y$ is a topological space and $\varphi(x)$ is closed for every $x \in X$, then the relation $\varphi$ is said to be closed-valued. If $Y$ is a vector space and $\varphi(x)$ is convex for every $x \in X$, then the relation $\varphi$ is said to be convex-valued.

A relation $\varphi$ from a set $X$ into a set $Y$ such that $\varphi(x) \neq \emptyset$ for every $x \in X$ is called a correspondence. Notice that a correspondence $\varphi: X \rightarrow Y$ can be considered as a function if $\varphi(x)$ contains exactly one element for every $x \in X$.

Now upper hemi-continuity of a correspondence is defined.

Definition 2.1 (Upper hemi-continuity) Let topological spaces $X$ and $Y$ and an element $\bar{x}$ of $X$ be given. A correspondence $\varphi: X \rightarrow Y$ is upper hemi-continuous at $\bar{x}$ if for every open set $V$ of $Y$ satisfying $\varphi(\bar{x}) \subset V$ there exists an open set $U$ of $X$ containing $\bar{x}$ such that $\varphi(U) \subset V$. The correspondence $\varphi$ is upper hemi-continuous if it is upper hemi-continuous at every $x \in X$.

Notice that a correspondence being both upper hemi-continuous and a function is a continuous function. Often it is possible to give easy characterizations of upper hemi-continuity.

Theorem 2.2 Let a subset $S$ of $\mathbb{R}^{m}$, a subset $T$ of $\mathbb{R}^{n}$, an element $\bar{x}$ of $S$, and a compact-valued correspondence $\varphi: S \rightarrow T$ be given. Then the correspondence $\varphi$ is upper hemi-continuous at $\bar{x}$ if and only if for every sequence $\left(x^{n}\right)_{n \in \mathbb{N}}$ in $S$ converging to $\bar{x}$ and every sequence $\left(y^{n}\right)_{n \in \mathbb{N}}$ in $T$ with $y^{n} \in \varphi\left(x^{n}\right)$, for all $n \in \mathbb{N}$, there exists a subsequence of $\left(y^{n}\right)_{n \in \mathbb{N}}$ converging to some element of $\varphi(\bar{x})$.

See Hildenbrand (1974), Theorem 1, page 24.

Theorem 2.3 Let a subset $S$ of $\mathbb{R}^{m}$, a compact subset $T$ of $\mathbb{R}^{n}$, an element $\bar{x}$ of $S$, and a compact-valued correspondence $\varphi: S \rightarrow T$ be given. Then the correspondence $\varphi$ is upper hemi-continuous at $\bar{x}$ if and only if for every sequence $\left(x^{n}\right)_{n \in \mathbb{N}}$ in $S$ converging to $\bar{x}$ and every 
sequence $\left(y^{n}\right)_{n \in \mathbb{N}}$ in $T$ with $y^{n} \in \varphi\left(x^{n}\right)$, for all $n \in \mathbb{N}$, converging to $\bar{y} \in \mathbb{R}^{n}$ it holds that $\bar{y} \in \varphi(\bar{x})$. The correspondence $\varphi$ is upper hemi-continuous if and only if the graph of $\varphi$ is closed in $S \times T$.

See Debreu (1959), 1.8.d, page 17.

Next, lower hemi-continuity of a correspondence is defined.

Definition 2.4 (Lower hemi-continuity) Let topological spaces $X$ and $Y$ and an element $\bar{x}$ of $X$ be given. A correspondence $\varphi: X \rightarrow Y$ is lower hemi-continuous at $\bar{x}$ if for every open set $V$ of $Y$ satisfying $\varphi(\bar{x}) \cap V \neq \emptyset$ there exists an open set $U$ of $X$ containing $\bar{x}$ such that $\varphi(x) \cap V \neq \emptyset$ for all $x \in U$. The correspondence $\varphi$ is lower hemi-continuous if it is lower hemi-continuous at every $x \in X$.

Often it is possible to give easy characterizations of lower hemi-continuity.

Theorem 2.5 Let a subset $S$ of $\mathbb{R}^{m}$, a subset $T$ of $\mathbb{R}^{n}$, and an element $\bar{x}$ of $S$ be given. Then the correspondence $\varphi$ is lower hemi-continuous at $\bar{x}$ if and only if for every sequence $\left(x^{n}\right)_{n \in \mathbb{N}}$ in $S$ converging to $\bar{x}$ and for every element $\bar{y}$ of $\varphi(\bar{x})$ there exists a sequence $\left(y^{n}\right)_{n \in \mathbb{N}}$ in $T$ such that $y^{n} \in \varphi\left(x^{n}\right)$, for all $n \in \mathbb{N}$, and $y^{n} \rightarrow \bar{y}$.

See Hildenbrand (1974), Theorem 2, page 27.

Finally, continuity of a correspondence is defined.

Definition 2.6 (Continuity) Let topological spaces $X$ and $Y$ and an element $\bar{x}$ of $X$ be given. A correspondence $\varphi: X \rightarrow Y$ is continuous at $\bar{x}$ if it is both upper and lower hemi-continuous at $\bar{x}$. The correspondence $\varphi$ is continuous if it is continuous at every $x \in X$.

Let $\varphi$ be a relation from a set $X$ into a set $Y$. The element $x$ of $X$ is called a fixed point of $\varphi$ if $x \in \varphi(x)$. Related to the concept of a fixed point is the notion of a zero point. Let $\varphi$ be a relation from a set $X$ into $\mathbb{R}^{m}$. The element $x$ of $X$ is called a zero point of $\varphi$ if $0 \in \varphi(x)$. As already mentioned, several fixed point theorems are essential for the theory developed in this monograph. The first one is known as Kakutani's fixed point theorem.

Theorem 2.7 (Kakutani's fixed point theorem) Let $S$ be a non-empty, compact, convex subset of $\mathbb{R}^{m}$ and let $\varphi: S \rightarrow S$ be a convex-valued correspondence such that the graph of $\varphi$ is closed in $S \times S$. Then there exists an element $x$ of $S$ such that $x \in \varphi(x)$.

See Kakutani (1941), Theorem 1, page 457, and Corollary of Theorem 1, page 458.

Notice that by Theorem 2.3 the correspondence $\varphi$ in Theorem 2.7 has a closed graph if and only if $\varphi$ is a compact-valued, upper hemi-continuous correspondence. If the correspondence $\varphi$ is a function, Brouwer's fixed point theorem is obtained (see Brouwer (1912)). Secondly and finally, an extension of Browder's fixed point theorem as formulated in Theorem 2 in Browder (1960) is given. The following theorem is a special case of Theorem 3 in Mas-Colell (1974). 
Theorem 2.8 (Browder's fixed point theorem) Let $S$ be a non-empty, compact, convex subset of $\mathbb{R}^{m}$ and let $\varphi:[0,1] \times S \rightarrow S$ be a convex-valued correspondence such that the graph of $\varphi$ is closed in $[0,1] \times S \times S$. Then the set $F_{\varphi}=\{(\lambda, x) \in[0,1] \times S \mid x \in \varphi(\lambda, x)\}$ contains a connected set $F_{\varphi}^{c}$ such that $(\{0\} \times S) \cap F_{\varphi}^{c} \neq \emptyset$ and $(\{1\} \times S) \cap F_{\varphi}^{c} \neq \emptyset$.

See Mas-Colell (1974), Theorem 3, page 230.

Notice that by Theorem 2.3 the correspondence $\varphi$ in Theorem 2.8 has a closed graph if and only if $\varphi$ is a compact-valued, upper hemi-continuous correspondence. For more on fixed point theorems and its applications to economics and game theory, the reader is referred to Border (1985).

\subsection{Differential topology}

This section is mainly based on Golubitsky and Guillemin (1973) and Jongen, Jonker, and Twilt (1983, 1986). For a nice introduction into the field of differential topology the reader is referred to Milnor (1965) and Guillemin and Pollack (1974).

Let $r \in \mathbb{N}$, an open set $U$ of $\mathbb{R}^{m}$, and a non-empty subset $T$ of $\mathbb{R}^{n}$ be given. A function $f: U \rightarrow T$ is called $r$ times continuously differentiable if all partial derivatives up to the order $r$ are continuous functions. The set of $r$ times continuously differentiable functions from $U$ into $T$ is denoted by $C^{r}(U, T)$. The set $C^{\infty}(U, T)$ is defined by $C^{\infty}(U, T)=\bigcap_{r \in \mathbb{N}} C^{r}(U, T)$. An element of $C^{\infty}(U, T)$ is called a smooth function. It follows easily that $C^{\infty}(U, T) \subset \cdots \subset$ $C^{1}(U, T) \subset C^{0}(U, T)$.

The following result is known as the inverse function theorem.

Theorem 2.9 (Inverse function theorem) For $r \in \mathbb{N}^{*}$, let an open subset $U$ of $\mathbb{R}^{m}$, an element $\bar{x}$ of $U$, and a function $f \in C^{r}\left(U, \mathbb{R}^{m}\right)$ be given. If $\partial_{x} f(\bar{x})$ is an invertible matrix, then there exist open sets $U^{1}$ and $U^{2}$ of $\mathbb{R}^{m}$ such that $\bar{x} \in U^{1}$, the function $g \in C^{r}\left(U^{1}, U^{2}\right)$, defined by $g(x)=f(x)$, for all $x \in U^{1}$, is injective and surjective, $g^{-1} \in C^{r}\left(U^{2}, U^{1}\right)$, and $\partial_{x} g^{-1}(g(\bar{x}))=$ $\left(\partial_{x} g(\bar{x})\right)^{-1}$. If $U^{1}$ and $U^{2}$ are open sets of $\mathbb{R}^{m}$ and $g$ is a function of $C^{r}\left(U^{1}, U^{2}\right)$ such that $g$ is injective, surjective, and $g^{-1} \in C^{r}\left(U^{2}, U^{1}\right)$, then $\partial_{x} g(\bar{x})$ is invertible for every $\bar{x} \in U^{1}$.

See Mas-Colell (1985), Theorem C.3.1, page 20.

Let $r \in \mathbb{N}^{*}, S \subset \mathbb{R}^{m}$ and $T \subset \mathbb{R}^{n}$ be given. A function $f: S \rightarrow T$ is called $r$ times continuously differentiable if for every element $x$ of $S$ there exists an open set $U$ of $\mathbb{R}^{m}$ containing $x$ and a function $g \in C^{r}\left(U, \mathbb{R}^{n}\right)$ such that $g(x)=f(x)$ for all $x \in S \cap U$. The set of $r$ times continuously differentiable functions from $S$ into $T$ is denoted by $C^{r}(S, T)$. A function $f: S \rightarrow T$ is called a $C^{r}$ diffeomorphism if $f$ is injective, surjective, $f \in C^{r}(S, T)$, and $f^{-1} \in C^{r}(T, S)$. The sets $S$ and $T$ are called $C^{r}$ diffeomorphic if there exists a $C^{r}$ diffeomorphism $f: S \rightarrow T$. Although 
the above definitions are of a local character, the following results show that they can be made global.

Theorem 2.10 For $r \in \mathbb{N}^{*}$, let a subset $S$ of $\mathbb{R}^{m}$, a subset $T$ of $\mathbb{R}^{n}$, and a function $f \in C^{r}(S, T)$ be given. Then there exists an open subset $U$ of $\mathbb{R}^{m}$ containing the set $S$ and there exists $a$ function $g \in C^{r}\left(U, \mathbb{R}^{n}\right)$ such that $g(x)=f(x)$, for all $x \in S$.

See Jongen, Jonker, and Twilt (1983), Lemma 3.1.5, page 103.

Intuitively, for some $k \in \mathbb{N}$, a $k$-dimensional manifold is a set which is locally like $\mathbb{R}^{k}$. The following definitions make this statement more precise.

Definition 2.11 (Topological manifold) For $k \in \mathbb{Z}_{+}$, a subset $X$ of $\mathbb{R}^{m}$ is a $k$-dimensional topological manifold if for every element $x$ of $X$ there exists an open set $U$ of $X$ containing $x$, an open set $V$ of $\mathbb{R}^{k}$, and an injective and surjective function $\phi: U \rightarrow V$ such that $\phi \in C^{0}(U, V)$ and $\phi^{-1} \in C^{0}(V, U)$, i.e. $\phi: U \rightarrow V$ is a homeomorphism.

Definition 2.12 ( $C^{r}$ manifold) For $k \in \mathbb{Z}_{+}$, for $r \in \mathbb{N}^{*}$, a subset $X$ of $\mathbb{R}^{m}$ is a $k$-dimensional $C^{r}$ manifold if for every element $x$ of $X$ there exists an open set $U$ of $X$ containing $x$, an open set $V$ of $\mathbb{R}^{k}$, and an injective and surjective function $\phi: U \rightarrow V$ such that $\phi \in C^{r}(U, V)$ and $\phi^{-1} \in C^{r}(V, U)$, i.e. $\phi: U \rightarrow V$ is a $C^{r}$ diffeomorphism.

The pair $(U, \phi)$ in Definition 2.12 is called a chart of $X$ around $x$ and $\left(V, \phi^{-1}\right)$ are called local $C^{r}$ coordinates for $U$. The function $\phi$ is called a $C^{r}$ coordinate system for $X$ around $x$.

Definition 2.13 (Piecewise $C^{r}$ manifold) For $k \in \mathbb{Z}_{+}$, for $r \in \mathbb{N}^{*}$, a subset $X$ of $\mathbb{R}^{m}$ is a $k$ dimensional piecewise $C^{r}$ manifold if $X$ is a $k$-dimensional topological manifold being a finite union of $C^{r}$ manifolds.

If $X$ is a $k$-dimensional topological manifold, a $k$-dimensional $C^{r}$ manifold, or a $k$-dimensional piecewise $C^{r}$ manifold, then the dimension of $X$ is said to be $k$. In Definition 2.13 it is allowed that the dimension of some of the $C^{r}$ manifolds whose union is equal to $X$ is less than $k$.

For $k \in \mathbb{Z}_{+}$, for $r \in \mathbb{N}^{*}$, let a $k$-dimensional $C^{r}$ manifold $X$, an element $\bar{x}$ of $X$, and a coordinate system $\phi$ of $X$ around $x$ be given. Then the set $\partial \phi^{-1}(\phi(\bar{x}))\left(\mathbb{R}^{k}\right)$ is called the tangent space of $X$ at $\bar{x}$ and is denoted by $T_{\bar{x}} X$. It can be shown that the set $T_{\bar{x}} X$ does not depend on the choice of the coordinate system $\phi$.

For $k \in \mathbb{Z}_{+}$, for $r \in \mathbb{N}^{*}$, a characterization of a $k$-dimensional $C^{r}$ manifold is given in the following theorem.

Theorem 2.14 For $k \in \mathbb{Z}_{+}$, for $r \in \mathbb{N}^{*}$, a subset $X$ of $\mathbb{R}^{m}$ is a $k$-dimensional $C^{r}$ manifold if and only if for every element $x$ of $X$ there exists a $C^{r}$ coordinate system $\phi: U \rightarrow V$ of $\mathbb{R}^{m}$ around $x$ satisfying $\phi(x)=0$ and $\phi(X \cap U)=\left\{y \in V \mid y_{i}=0, \forall i \in I_{m-k}\right\}$.

See Jongen, Jonker, and Twilt (1983), Theorem 3.11, page 89. 
Notice that, by definition, the function $\phi: U \rightarrow V$ in Theorem 2.14 is a $C^{r}$ coordinate system of $\mathbb{R}^{m}$ around $x$ if $x \in U, U$ and $V$ are open sets of $\mathbb{R}^{m}, \phi$ is injective and surjective, $\phi \in C^{r}(U, V)$, and $\phi^{-1} \in C^{r}(V, U)$.

Definition 2.15 (Manifold with generalized boundary) For $k \in \mathbb{Z}_{+}$, for $r \in \mathbb{N}^{*}$, a subset $X$ of $\mathbb{R}^{m}$ is a $k$-dimensional $C^{r}$ manifold with generalized boundary $(M G B)$ if for every element $x$ of $X$ there exists a $C^{r}$ coordinate system $\phi: U \rightarrow V$ of $\mathbb{R}^{m}$ around $x$ and an integer $\ell(x)$, $0 \leq \ell(x) \leq k$, satisfying $\phi(x)=0$ and

$$
\phi(X \cap U)=\left\{y \in V \mid y_{i}=0, \forall i \in I_{m-k}, \text { and } y_{i} \geq 0, \forall i \in I_{m-k+\ell(x)} \backslash I_{m-k}\right\} .
$$

If $X$ is a $k$-dimensional $C^{r}$ manifold with generalized boundary, then the dimension of $X$ is said to be $k$. It follows that the dimension $k$, for every $\operatorname{MGB} X$, and the integer $\ell(x)$, for every element $x$ of $X$, are independent of the choice of the coordinate system. Intuitively, a $k$-dimensional manifold with generalized boundary is in a neighborhood of $\bar{x} \in X$ like the set $\left\{x \in \mathbb{R}^{k} \mid x_{i} \geq 0, \forall i \in I_{\ell(\bar{x})}\right\}$.

For $k \in \mathbb{Z}_{+}$, for $r \in \mathbb{N}^{*}$, let the set $X$ be a $k$-dimensional $C^{r}$ MGB. Define the set $B^{l}(X)$ by

$$
B^{l}(X)=\{x \in X \mid \ell(x)=l\}
$$

for all $l \in I_{k}^{0}$. The set $B^{0}(X)$ is called the relative interior of $X$. The set $X \backslash B^{0}(X)$ is called the relative boundary of $X$. These definitions of relative interior and relative boundary are consistent with the corresponding definitions in Section 2.2 for arcs. In case $X=B^{0}(X)$ it follows that the set $X$ is a $k$-dimensional $C^{r}$ manifold by Theorem 2.14. If $X=B^{0}(X) \cup B^{1}(X)$, then the set $X$ is called manifold with boundary. The following theorem yields that the relative boundary of a manifold with boundary is a manifold.

Theorem 2.16 For $k \in \mathbb{Z}_{+}, r \in \mathbb{N}^{*}$, let the set $X$ be a $k$-dimensional $C^{r} M G B$. For every $l \in I_{k}^{0}$, the set $B^{l}(X)$ is a $(k-l)$-dimensional $C^{r}$ manifold.

See Jongen, Jonker, and Twilt (1983), Lemma 3.1.4, page 98.

Compact 1-dimensional MGB's have a particular nice structure, as follows from the following theorem.

Theorem 2.17 For $r \in \mathbb{N}^{*}$, let the set $X$ be a compact 1-dimensional $C^{r} M G B$. Then the set $X$ has a finite number of components, each being $C^{r}$ diffeomorphic to either the unit circle in $\mathbb{R}^{2}$, or the closed unit interval in $\mathbb{R}$.

See Mas-Colell (1985), H.6, page 35.

An interesting class of sets are the so-called regular constraint sets. Let $U$ be an open set of $\mathbb{R}^{m}$ and let, for some $n^{1} \in \mathbb{Z}_{+}$, for some $n^{2} \in \mathbb{Z}_{+}$, functions $\tilde{g}_{j}: U \rightarrow \mathbb{R}$, for all $j \in I_{n^{1}}$, and $\tilde{h}_{j}: U \rightarrow \mathbb{R}$, for all $j \in I_{n^{2}}$, be given. Define the set $M[\tilde{g}, \tilde{h}]$ by

$$
M[\tilde{g}, \tilde{h}]=\left\{x \in U \mid \tilde{g}_{j}(x)=0, \forall j \in I_{n^{1}} \text {, and } \tilde{h}_{j}(x) \geq 0, \forall j \in I_{n^{2}}\right\} .
$$


For every element $x$ of $U$, define the set $J^{0}(x)=\left\{j \in I_{n^{2}} \mid \tilde{h}_{j}(x)=0\right\}$. In the above definitions $n^{1}$ and $n^{2}$ are allowed to be zero, in which case $\tilde{g}$ and $\tilde{h}$ are denoted by $\emptyset$, respectively.

Definition 2.18 (Regular constraint system and regular constraint set) For $n^{1}, n^{2} \in \mathbb{Z}_{+}$, for $r \in \mathbb{N}^{*}$, let $U$ be an open set of $\mathbb{R}^{m}$ and let $\tilde{g}_{j}: U \rightarrow \mathbb{R}$, for all $j \in I_{n^{1}}$, and $\tilde{h}_{j}: U \rightarrow \mathbb{R}$, for all $j \in I_{n^{2}}$, be $C^{r}$ functions. The pair of functions $(\tilde{g}, \tilde{h})$ is a $C^{r}$ regular constraint system if for every element $\bar{x}$ of $M[\tilde{g}, \tilde{h}]$ the set

$$
\left\{\partial_{x} \tilde{g}_{j}(\bar{x})^{\top} \mid j \in I_{n^{1}}\right\} \cup\left\{\partial_{x} \tilde{h}_{j}(\bar{x})^{\top} \mid j \in J^{0}(\bar{x})\right\}
$$

is an independent set. The set $S$ is a $C^{r}$ regular constraint set $(R C S)$ if there exists a $C^{r}$ regular constraint system $(\tilde{g}, \tilde{h})$ such that $S=M[\tilde{g}, \tilde{h}]$.

Theorem 2.19 For $r \in \mathbb{N}^{*}$, let the subset $X$ of $\mathbb{R}^{m}$ be a $C^{r} R C S$ and let the pair of functions $(\tilde{g}, \tilde{h})$ be a $C^{r}$ regular constraint system such that $M[\tilde{g}, \tilde{h}]=X$, where $\tilde{g}$ has $n^{1}$ components. Then $X$ is a k-dimensional $C^{r} M G B$ with $k=m-n^{1}$. Moreover, $\ell(x)=\left|J^{0}(x)\right|$ for every element $x$ of $X$.

See Jongen, Jonker, and Twilt (1983), Lemma 3.1.2, page 94.

Theorem 2.19 shows that the dimension of a non-empty RCS is well-defined. In many cases Theorem 2.19 can be used to show that a certain set is an MGB.

Next, the tangent space of a manifold with generalized boundary is defined.

Definition 2.20 (Tangent space) For $k \in \mathbb{Z}_{+}$, for $r \in \mathbb{N}^{*}$, let the set $X$ be a $k$-dimensional $C^{r}$ MGB. Let $\bar{x}$ be an element of $X$ and let the function $\phi$ be a $C^{r}$ coordinate system for $\mathbb{R}^{m}$ around $\bar{x}$ with the properties given in Definition 2.15. The tangent space of $X$ at $\bar{x}$, denoted by $T_{\bar{x}} X$, is the set $\partial \phi^{-1}(0)\left(\{0\} \times \mathbb{R}^{k}\right)$.

It can be shown that the tangent space $T_{\bar{x}} X$ as defined in Definition 2.20 does not depend on the choice of the coordinate system. Moreover, in case the set $X \backslash B^{0}(X)$ is empty, the definition of the tangent space as given in Definition 2.20 coincides with the definition given before. Since $\partial \phi^{-1}(0)$ is a $C^{\infty}$ diffeomorphism, it holds by Theorem 2.9 that $\partial_{x} \phi^{-1}(0)$ is an invertible matrix and therefore $T_{\bar{x}} X$ is a $k$-dimensional vector space.

In case a set $X$ is an RCS the following theorem gives an easy way to determine the tangent space of $X$ at an element of $X$.

Theorem 2.21 For $k \in \mathbb{Z}_{+}$, for $r \in \mathbb{N}^{*}$, let the subset $X$ of $\mathbb{R}^{m}$ be a $k$-dimensional $C^{r}$ RCS, let $\bar{x}$ be an element of $X$, and let the pair of functions $(\tilde{g}, \tilde{h})$ be a regular constraint system such that $X=M[\tilde{g}, \tilde{h}]$. Then $T_{\bar{x}} X=\left\{x \in \mathbb{R}^{m} \mid \partial \tilde{g}(\bar{x})(x)=0\right\}$.

See Jongen, Jonker, and Twilt (1983), Lemma 3.2.2, page 111. 
For $r \in \mathbb{N}^{*}$, let the subsets $X$ of $\mathbb{R}^{m}$ and $Y$ of $\mathbb{R}^{n}$ be $C^{r}$ manifolds, let $\bar{x}$ be an element of $X$, and let $f$ be a function of $C^{r}(X, Y)$. Let $U$ be an open set of $\mathbb{R}^{m}$ such that $X \subset U$ and let the function $g \in C^{r}\left(U, \mathbb{R}^{n}\right)$ be such that $g(x)=f(x)$, for all $x \in X$. Such a function $g$ exists by Theorem 2.10. It can be shown that $\partial g(\bar{x})_{\mid T_{\bar{x}} X}$ is a function from $T_{\bar{x}} X$ into $T_{f(\bar{x})} Y$. Moreover, the function $\partial g(\bar{x})_{\mid T_{\bar{x}} X}$ does not depend on the choice of the function $g$. The derivative of $f$ at $\bar{x}$, denoted by $\partial f(\bar{x})$, is defined by $\partial f(\bar{x})=\partial g(\bar{x})_{\mid T_{\bar{x}} X}$. The element $\bar{x}$ is called a regular point of $f$ if $\partial f(\bar{x})\left(T_{\bar{x}} X\right)=T_{f(\bar{x})} Y$. Otherwise $\bar{x}$ is called a critical point of $f$. Let an element $\bar{y}$ of $Y$ be given. The element $\bar{y}$ is called a critical value of $f$ if it is the image of a critical point of $f$. Otherwise $\bar{y}$ is called a regular value of $f$. Notice that every element $y$ of $Y \backslash f(X)$ is a regular value of $f$.

Let $C^{1}$ manifolds $X, Y$, and $Z, Z$ being a subset of $Y$, an element $\bar{x}$ of $X$, and a function $f \in C^{1}(X, Y)$ be given. The function $f$ is said to intersect $Z$ transversally at $\bar{x} \in X$, denoted by $f \pitchfork Z$ at $\bar{x}$, if

$$
f(\bar{x}) \notin Z, \text { or } f(\bar{x}) \in Z \text { and } T_{f(\bar{x})} Z+\partial f(\bar{x})\left(T_{\bar{x}} X\right)=T_{f(\bar{x})} Y .
$$

The function $f$ is said to intersect $Z$ transversally if $f \pitchfork Z$ at every $x \in X$. The following theorem follows always immediately form the definition of transversality.

Theorem 2.22 For $k^{1}, k^{2}, k^{3} \in \mathbb{Z}_{+}$, for $r \in \mathbb{N}^{*}$, let a $k^{1}$-dimensional $C^{1}$ manifold $X$, a $k^{2}$ dimensional $C^{1}$ manifold $Y$, and a $k^{3}$-dimensional $C^{1}$ manifold $Z, Z$ being a subset of $Y$, be given, and let $f \in C^{1}(X, Y)$ be such that $f \mp Z$. If $k^{1}-k^{2}+k^{3}<0$, then $f^{-1}(Z)=\emptyset$.

See Golubitsky and Guillemin (1973), Proposition 4.2, page 51.

The following result is complementary to Theorem 2.22.

Theorem 2.23 For $k^{1}, k^{2}, k^{3} \in \mathbb{Z}_{+}$, for $r \in \mathbb{N}^{*}$, let a $k^{1}$-dimensional $C^{r}$ manifold $X$, a $k^{2}$ dimensional $C^{r}$ manifold $Y$, and a $k^{3}$-dimensional $C^{r}$ manifold $Z, Z$ being a subset of $Y$, be given, and let $f \in C^{r}(X, Y)$ be such that $f \mp Z$. If $k^{1}-k^{2}+k^{3} \geq 0$, then $f^{-1}(Z)$ is a $\left(k^{1}-k^{2}+k^{3}\right)$-dimensional $C^{r}$ manifold.

See Mas-Colell (1985), I.2.1, page 43.

The following result is an easy corollary to Theorems 2.22 and 2.23.

Theorem 2.24 For $k^{1}, k^{2} \in \mathbb{Z}_{+}$, for $r \in \mathbb{N}^{*}$, let a $k^{1}$-dimensional $C^{r}$ manifold $X$, a $k^{2}$ dimensional $C^{r}$ manifold $Y$, and a function $f \in C^{r}(X, Y)$ be given. Let the element $\bar{y}$ of $Y$ be a regular value of $f$. If $k^{1}-k^{2}<0$, then $f^{-1}(\{\bar{y}\})=\emptyset$, and if $k^{1}-k^{2} \geq 0$, then $f^{-1}(\{\bar{y}\})$ is a $\left(k^{1}-k^{2}\right)$-dimensional $C^{r}$ manifold.

See Mas-Colell (1985), H.2.2, page 38. 
For Theorem 2.26 the notion of Lebesgue measure zero in $X$ for a subset of a manifold $X$ needs to be defined. For $k \in \mathbb{Z}_{+}$, for $r \in \mathbb{N}^{*}$, let the subset $X$ of $\mathbb{R}^{m}$ be a $k$-dimensional $C^{r}$ manifold and let $S$ be a subset of $X$. Then the set $S$ is said to have Lebesgue measure zero in $X$ if there exists a countable cover $\left\{U^{n} \mid n \in \mathbb{N}\right\}$ of $S$ and charts $\left(U^{n}, \phi^{n}\right)$, for all $n \in \mathbb{N}$, such that $\phi^{n}\left(U^{n} \cap S\right)$ has Lebesgue measure zero for every $n \in \mathbb{N}$. In case $X$ is an $m$-dimensional $C^{r}$ manifold, being a subset of $\mathbb{R}^{m}$, Theorem 2.25 will be used to show that the notions of Lebesgue measure zero and Lebesgue measure zero in $X$ coincide.

Theorem 2.25 Let a subset $S$ of $\mathbb{R}^{m}$ with Lebesgue measure zero and a function $f \in C^{1}\left(S, \mathbb{R}^{m}\right)$ be given. Then $f(S)$ has Lebesgue measure zero.

See Golubitsky and Guillemin (1973), Proposition 1.3, page 30.

For $r \in \mathbb{N}^{*}$, let the subset $X$ of $\mathbb{R}^{m}$ be an $m$-dimensional $C^{r}$ manifold and let $S$ be a subset of $X$. Using Theorem 2.25 it follows that if the set $S$ has Lebesgue measure zero, then $S$ has Lebesgue measure zero in $X$. Now assume that the set $S$ has Lebesgue measure measure zero in $X$. Then there exists a countable cover $\left\{U^{n} \mid n \in \mathbb{N}\right\}$ of $S$ and charts $\left(U^{n}, \phi^{n}\right)$, for all $n \in \mathbb{N}$, such that the set $V^{n}=\phi^{n}\left(U^{n} \cap S\right)$ is of Lebesgue measure zero for every $n \in \mathbb{N}$. For every $n \in \mathbb{N}$, since $\left(\phi^{n}\right)^{-1} \in C^{r}\left(V^{n}, \mathbb{R}^{m}\right)$, it follows, from Theorem 2.25 and the fact that a subset of a set with Lebesgue measure zero has Lebesgue measure, that $\left(\phi^{n}\right)^{-1}\left(V^{n}\right)$ has Lebesgue measure zero. Consequently, the set $\bigcup_{n \in \mathbb{N}}\left(\phi^{n}\right)^{-1}\left(V^{n}\right)=S$ has Lebesgue measure zero.

Theorem 2.26 (Transversality theorem) For $k^{1}, k^{2}, k^{3} \in \mathbb{Z}_{+}$, for $r \in \mathbb{N}^{*}$, let a $k^{1}$-dimensional $C^{r}$ manifold $X^{1}$, a $C^{r}$ manifold $X^{2}$, a $k^{2}$-dimensional $C^{r}$ manifold $Y$, a $k^{3}$-dimensional $C^{r}$ manifold $Z$, being a subset of $Y$, and a function $f \in C^{r}\left(X^{1} \times X^{2}, Y\right)$ be given, with $r \geq$ $\max \left\{1, k^{1}-k^{2}+k^{3}\right\}$. For every $x^{2} \in X^{2}$, define a function $f^{x^{2}} \in C^{r}\left(X^{1}, Y\right)$ by $f^{x^{2}}\left(x^{1}\right)=$ $f\left(x^{1}, x^{2}\right)$, for all $x^{1} \in X^{1}$. Then $f \pitchfork Z$ implies $f^{x^{2}} \pitchfork Z$, except for a subset of $X^{2}$ having Lebesgue measure zero in $X^{2}$.

See Mas-Colell (1985), I.2.2, page 45. 


\section{Part I}

Normal Form Games 



\section{Chapter 3}

\section{Computation of Equilibria}

The literature on the computation of Nash equilibria in $n$-person games is dominated by simplicial methods. This chapter is based on Herings and Peeters (2001a), the first paper to introduce a globally convergent algorithm that fully exploits the differentiability present in the problem. It presents an everywhere differentiable homotopy to do the computations. The homotopy path can therefore be followed by several numerical techniques. Moreover, instead of computing some Nash equilibrium, the algorithm is constructed in such a way that it computes the Nash equilibrium selected by the tracing procedure of Harsanyi and Selten. As a consequence of the proofs it follows that for a generic game the tracing procedure defines a unique feasible path. The numerical performance of the algorithm is illustrated by means of several examples.

\subsection{Introduction}

During the past few years, many research fields in which conflicts between agents arise have benefited from the introduction of game theoretic tools. A further use of game theory may stagnate for several reasons. One is that there may be a great number of solutions to a given game. Recent work of McLennan (1999) shows that the number of Nash equilibria in normal form games of modest size is huge on average. For instance, the mean number of Nash equilibria in a game with 4 agents, each having 6 strategies, is estimated to be 2.037, with a standard error of the estimation of 66. Nevertheless, computation of just a single Nash equilibrium may prove to be hard in many games of interest. This point is expressed as follows in van Damme (1995):

"In the last two decades, game theoretic methods have become more and more important in economics and the other social sciences. Many scientific papers in these areas have the following basic structure: A problem is modeled as a game, the game is analyzed by computing its equilibria, and the properties of the latter are translated back into insights relevant to the original problem. ... It has been found that the tools may not be powerful enough ... For example, many models admit a vast 
multiplicity of equilibrium outcomes so that the predictive power of game theoretic analysis is limited. To increase understanding, it may, hence, be necessary to perfect the tools.".

A tool to increase understanding has been suggested in Harsanyi and Selten (1988), who introduce the linear tracing procedure as a means for equilibrium selection in finite $n$-person games. The linear tracing procedure is a mathematical construct that adjusts arbitrary prior beliefs into equilibrium beliefs. First, the players optimize by playing best responses against identical prior beliefs concerning the play of the other players. Next, they observe that their beliefs are not met and they subsequently update their beliefs and react optimally there upon. This updating of beliefs continues until equilibrium beliefs for the game have been found. Although the term "procedure" suggests a numerical approach, the tracing procedure itself is a non-constructive method.

From here a second problem for further progress in the applications of game theory to stagnate is found. Namely, the difficulties involved in solving games of interest. The view expressed in Judd (1997) that in general computational methods will serve an important role in the further development of economic theory, is also applicable in the progress of game theory.

The aim of this chapter is to present an algorithm to compute a Nash equilibrium for an arbitrary finite $n$-person game in normal form. Since there is a vast multiplicity of Nash equilibria in many games, it is also essential to provide an algorithm that performs the task of equilibrium selection (actually, each algorithm to compute for a sample equilibrium selects by definition, but here is meant to select on equilibria satisfying certain refinement properties). In particular, the algorithm has to compute the Nash equilibrium selected by the linear tracing procedure. Finally, the algorithm should be fast in that it allows for the computation of a Nash equilibrium for nontrivial games within reasonable time limits.

The first procedures for finding an equilibrium in $n$-person games were developed simultaneously and independently by Rosenmüller (1971) and Wilson (1971). Both methods are generalizations of the procedure of Lemke and Howson (1964) for bimatrix games. Although these methods are not directly suitable for computational purposes, their common result, the existence of a non-linear path leading to an equilibrium, was a very important step towards an implementable algorithm as developed by Garcia, Lemke and Lüthi (1973). Later, more efficient algorithms were proposed in van der Laan, Talman and Van der Heyden (1987) and Doup, van der Laan and Talman (1987). A problem of these algorithms is that they calculate only an approximation of a sample Nash equilibrium and do not bother about the game-theoretic underpinning of the calculated equilibrium.

The case of 2-person games is special in that the exact calculation of a Nash equilibrium is possible due to the linear structure of such a game. For this class of games, the algorithm of van den Elzen and Talman (1999) computes the Nash equilibrium selected by the linear tracing 
procedure. The generalization to $n$-person games is covered by the algorithm presented in Herings and van den Elzen (2002). That algorithm, as well as all the other algorithms discussed above, is a simplicial method. Simplicial methods solve a non-linear equilibrium problem by solving a piecewise linear approximation of the problem. All known algorithms that have been shown to converge to a Nash equilibrium for a generic $n$-person game, are simplicial methods. A drawback of these methods is that they do not exploit the differentiable structure that is present in $n$-person games. For an excellent survey of methods to compute Nash equilibria in games, see McKelvey and McLennan (1996).

In this chapter an algorithm is proposed that is shown to converge to a Nash equilibrium for a generic $n$-person game. This algorithm is the first to exploit the differentiability present in games to the full extent. The variables are transformed in the way as proposed in Garcia and Zangwill (1981). This chapter contains the first rigorous proof that after the transformation differentiability results. The algorithm also solves the equilibrium selection problem in that it computes the Nash equilibrium selected by the linear tracing procedure.

The algorithm belongs to the class of homotopy methods. The formulation as a differentiable homotopy makes it possible to apply standard path-following techniques that are available in professionally programmed software. This makes implementation on a computer an easy exercise.

As a by-product of the convergence proof, an elegant and clean proof of the theorem in Harsanyi (1975) that for a generic game the linear tracing procedure yields a path leading to a unique Nash equilibrium and of the theorem in Rosenmüller (1971), Wilson (1971), and Harsanyi (1973) that for a generic game the number of Nash equilibria is odd is obtained.

This chapter is organized as follows. Some notations and the definition of the linear tracing procedure are given in Section 3.2. In Section 3.3, the properties of the linear tracing procedure are studied. It is shown that for almost every game, the linear tracing procedure is formed by a finite union of arcs and loops. Using a well-chosen transformation of variables, the linear tracing procedure is described by the zeros of an everywhere differentiable homotopy function in Section 3.4. Section 3.5 discusses the implementation of the homotopy algorithm and gives some numerical results for randomly generated games. Section 3.6 summarizes. Section 3.7 contains some proofs to theorems arising in the preceding sections.

\subsection{The linear tracing procedure}

A finite $n$-person game in normal form $\Gamma$ is given by a tuple $\left\langle N,\left\{S^{i}\right\}_{i \in N},\left\{u^{i}\right\}_{i \in N}\right\rangle$, with $S^{i}$ and $u^{i}: S \rightarrow \mathbb{R}$ the set of pure strategies and the payoff function of player $i$, respectively. The set of players is denoted by $N=\{1, \ldots, n\}$. The set $S=S^{1} \times \cdots \times S^{n}$ denotes the set of pure strategy combinations. Player $i$ has $m^{i}$ pure strategies. The total number of pure strategies is given by 
$m^{*}=\sum_{i \in N} m^{i}$; the total number of pure strategy combinations is given by $m=\prod_{i \in N} m^{i}$. The strategies of the players are numbered such that pure strategy $j \in\left\{1, \ldots, m^{i}\right\}$ of player $i$ is denoted by $s_{j}^{i}$. The union of the pure strategies over all players is given by $S^{*}=\bigcup_{i \in N} S^{i}$.

A mixed strategy of player $i$ is a probability distribution on $S^{i}$. The set of all probability distributions on $S^{i}$ is identified with $\Sigma^{i}$. For $\sigma^{i} \in \Sigma^{i}$, the probability assigned to pure strategy $s_{j}^{i}$ is given by $\sigma_{j}^{i}$. The strategy space of the game is therefore equal to $\Sigma=\chi_{i \in N} \Sigma^{i}$. Note that the dimension of $\Sigma$ equals $m^{*}-n$. Given a mixed strategy combination $\sigma \in \Sigma$ and a strategy $\bar{\sigma}^{i} \in \Sigma^{i}$, the mixed strategy that results from replacing $\sigma^{i}$ by $\bar{\sigma}^{i}$ is denoted by $\left(\sigma^{-i}, \bar{\sigma}^{i}\right)$. If a mixed strategy combination $\sigma \in \Sigma$ is played, then the probability $\sigma(s)$ that the pure strategy combination $s=\left(s_{j^{1}}^{1}, \ldots, s_{j^{n}}^{n}\right)$ occurs, is given by $\sigma(s)=\prod_{i \in N} \sigma_{j^{i}}^{i}$ and the expected payoff of player $i$ by $u^{i}(\sigma)=\sum_{s \in S} \sigma(s) u^{i}(s)$. The class of all noncooperative games is denoted by $\mathcal{G}$.

A mixed strategy combination $\sigma \in \Sigma$ is said to be a Nash equilibrium of game $\Gamma$ if $\sigma^{i}$ is a best response against $\sigma^{-i}$ for all $i \in N$. The set of Nash equilibria of game $\Gamma$ is denoted by $\operatorname{NE}(\Gamma)$ and is nonempty (Nash (1951)).

The equilibrium selection theory as presented in Harsanyi and Selten (1988) has the nice feature that it selects a unique Nash equilibrium for every game. The Harsanyi-Selten theory is to a large extent based on the linear tracing procedure, a mathematical procedure introduced in Harsanyi (1975) that yields, only dependent on initial beliefs, a unique Nash equilibrium for almost every game. To select the unique Nash equilibrium for the remaining measure zero set of games, Harsanyi and Selten use a construct called the logarithmic tracing procedure. The linear tracing procedure is used repeatedly in their equilibrium selection theory to find a unique solution of so-called basic games and to define risk-dominance relationships between Nash equilibria.

The linear tracing procedure models a process of convergent expectations by which rational players will come to adopt, and expect each other to adopt, a particular Nash equilibrium as a solution for a given game. Before applying the tracing procedure, every player is assumed to have a subjective probability distribution expressing his expectation about the strategic choices of the other players. Each player is assumed to use the same theory to determine his subjective probability distributions. Therefore, all players have the same expectations about the other players. This common subjective probability distribution is called the prior. In the naive Bayesian approach, all players choose best responses to their priors and would in this way reach a strategy-combination that does not constitute a Nash equilibrium in general. In the linear tracing procedure, the information on the best responses is only gradually fed back into the expectations of the players. As the linear tracing procedure proceeds, both the priors and their best responses will gradually change until both converge to some Nash equilibrium of the game.

Each generically convergent algorithm to compute a Nash equilibrium can be interpreted as a procedure that selects an equilibrium, but will in fact be a completely arbitrary selection procedure. Harsanyi-Selten's procedure, on the other hand, is comprehensively studied and finds 
its origin in the search for an equilibrium selection method. Therefore, it is attractive to have an algorithm which computes the equilibrium selected by the Harsanyi-Selten theory.

Consider some $n$-person game $\Gamma$ and some prior $p \in \Sigma$. For every $t \in[0,1]$, the linear tracing procedure generates a Nash equilibrium of the game $\Gamma^{t}=\left\langle N,\left\{S^{i}\right\}_{i \in N},\left\{v^{i}(t)\right\}_{i \in N}\right\rangle$, where the payoff function $v^{i}(t): S \rightarrow \mathbb{R}$ of player $i$ is defined by

$$
v^{i}(t ; s)=t u^{i}(s)+(1-t) u^{i}\left(p^{-i}, s^{i}\right) .
$$

The game $\Gamma^{0}$ corresponds to a trivial game, where all players believe that all their opponents play with probability 1 according to the prior belief. The game $\Gamma^{1}$ coincides with the original game $\Gamma$. A best response against a strategy combination $\sigma \in \Sigma$ in the game $\Gamma^{t}$ corresponds to a best response against the probability distribution $t[\sigma]+(1-t)[p]$ on $S$ in the game $\Gamma$. The latter probability distribution does in general not belong to $\Sigma$, since it may be correlated.

The linear tracing procedure links a Nash equilibrium of the game $\Gamma^{0}$ to a Nash equilibrium of $\Gamma^{1}$. The set of all Nash equilibria related to the games $\Gamma^{t}, t \in[0,1]$, is denoted by

$$
\mathcal{L}(\Gamma, p)=\left\{(t, \sigma) \in[0,1] \times \Sigma \mid \sigma \in \mathrm{NE}\left(\Gamma^{t}\right)\right\} .
$$

The linear tracing procedure is said to be feasible if there exists a path in $\mathcal{L}(\Gamma, p)$ connecting a best response against the prior to a Nash equilibrium of the game $\Gamma$, i.e. there exists a continuous function $\gamma:[0,1] \rightarrow \mathcal{L}(\Gamma, p)$ such that $\gamma(0) \in \mathcal{L}(\Gamma, p) \cap(\{0\} \times \Sigma)$ and $\gamma(1) \in \mathcal{L}(\Gamma, p) \cap(\{1\} \times \Sigma)$. In general there may be many trajectories $\gamma([0,1])$ that link a Nash equilibrium of $\Gamma^{0}$ to a Nash equilibrium of $\Gamma^{1}$. If this trajectory is unique, then the linear tracing procedure is said to be welldefined. If the linear tracing procedure is well-defined, then it selects a unique Nash equilibrium of the game $\Gamma$.

Schanuel, Simon and Zame (1991) have shown that the logarithmic tracing procedure connects the best responses to the prior beliefs to exactly one Nash equilibrium. From this result, the feasibility of the linear tracing procedure follows by an easy limit argument. The proofs related to the logarithmic tracing procedure use heavy mathematical machinery from the field of algebraic geometry. Herings (2000) gives two very short proofs of the feasibility of the linear tracing procedure that do not involve the logarithmic tracing procedure.

The linear tracing procedure is shown to be well-defined for a generic $n$-person game by Harsanyi (1975). His result is that given any prior distribution almost all $n$-person noncooperative games give rise to a well-defined linear tracing procedure. As a by-product of the convergence proof of the algorithm presented, a clean and elegant proof of Harsanyi's result is obtained.

\subsection{A piecewise differentiable approach}

From the previous section it is known that the linear tracing procedure contains a feasible path. The strategy of the algorithm is to end up in a Nash equilibrium of the game by following that 
path. A simplicial method that needs very little structure of the path is implemented in Herings and van den Elzen (2002). Here, path following methods that exploit the differentiability of the path are used. Unfortunately, the feasible path displays serious non-differentiabilities for most games, which makes the computation of Nash equilibria very difficult. This section analyzes the differentiability properties of $\mathcal{L}(\Gamma, p)$ and reveals that, although not necessarily everywhere differentiable, it has a lot of structure.

Let a subset $B^{*}$ of $S^{*}$ be given with the property that for every player $i$ there is at least one pure strategy $s_{j}^{i}$ in $B^{*}$, so $B^{*} \cap S^{i} \neq \emptyset$ for every player $i$. Such a set $B^{*}$ is called admissible. The sets $B^{*}$ are used to decompose $\mathcal{L}(\Gamma, p)$ in subsets $\mathcal{L}\left(\Gamma, p, B^{*}\right)$, each having a differentiable manifold structure. The set $\mathcal{L}\left(\Gamma, p, B^{*}\right)$ contains those elements of $\mathcal{L}(\Gamma, p)$ where only strategies in $B^{*}$ are played with positive probability. It is defined by

$$
\begin{aligned}
\mathcal{L}\left(\Gamma, p, B^{*}\right)=\{(t, \sigma) \in \mathcal{L}(\Gamma, p) \mid & s_{j}^{i} \notin B^{*} \Rightarrow \sigma_{j}^{i}=0 \\
s_{j}^{i} & \left.\in B^{*} \Rightarrow s_{j}^{i} \in \operatorname{argmax}_{s_{\ell}^{i} \in S^{i}} v^{i}\left(t ; \sigma^{-i}, s_{\ell}^{i}\right)\right\} .
\end{aligned}
$$

It follows that

$$
\mathcal{L}(\Gamma, p)=\bigcup_{B^{*}} \mathcal{L}\left(\Gamma, p, B^{*}\right) .
$$

Two sets $\mathcal{L}\left(\Gamma, p, B^{*}\right)$ and $\mathcal{L}\left(\Gamma, p, \bar{B}^{*}\right)$ with $B^{*} \neq \bar{B}^{*}$ can only have a point $(t, \sigma)$ in common if there is a player $i$ and a strategy $s_{j}^{i}$ of player $i$ such that $\sigma_{j}^{i}=0$ and $s_{j}^{i}$ is an element of $\operatorname{argmax}_{s_{\ell}^{i} \in S^{i}} v^{i}\left(t ; \sigma^{-i}, s_{\ell}^{i}\right)$, so strategy $s_{j}^{i}$ is a best response to $\sigma^{-i}$ that is played with probability zero.

To analyze the structure of $\mathcal{L}(\Gamma, p)$ and the sets $\mathcal{L}\left(\Gamma, p, B^{*}\right)$, systems of equalities and inequalities whose solutions characterize these sets are designed. The Kuhn-Tucker first-order conditions, which provide necessary and sufficient conditions for $\sigma^{i}$ to be a best response against $\bar{\sigma}^{-i}$ in the game $\Gamma^{\bar{t}}$, are given by

$$
\begin{aligned}
& v^{i}\left(\bar{t} ; \bar{\sigma}^{-i}, s_{j}^{i}\right)+\lambda_{j}^{i}-\mu^{i}=0, \quad\left(s_{j}^{i} \in S^{i}\right), \\
& \lambda_{j}^{i} \geq 0, \quad \sigma_{j}^{i} \geq 0, \quad \lambda_{j}^{i} \sigma_{j}^{i}=0, \quad\left(s_{j}^{i} \in S^{i}\right), \\
& \sum_{s_{j}^{i} \in S^{i}} \sigma_{j}^{i}-1=0 .
\end{aligned}
$$

Here, $\lambda_{j}^{i}$ is the shadowprice of playing strategy $s_{j}^{i}$, i.e. the disutility of a marginal increase in the probability $\sigma_{j}^{i}$ by which pure strategy $s_{j}^{i}$ is played. If $\sigma_{j}^{i}>0$, then $s_{j}^{i}$ is a best response to $\bar{\sigma}^{-i}$ in game $\Gamma^{\bar{t}}$, which implies $\lambda_{j}^{i}=0$. It follows that $\mu^{i}$ is equal to the payoff of player $i$ in game $\Gamma^{\bar{t}}$. The last equality, $\sum_{s_{j}^{i} \in S^{i}} \sigma_{j}^{i}-1=0$, makes sure that $\sigma^{i}$ is a member of $\Sigma^{i}$.

Given an admissible subset $B^{*}$, we define $\mathcal{O}\left(\Gamma, p, B^{*}\right)$ as the set of solutions $(t, \sigma, \lambda, \mu)$ to the following system of equalities and inequalities:

$$
v^{i}\left(t ; \sigma^{-i}, s_{j}^{i}\right)+\lambda_{j}^{i}-\mu^{i}=0, \quad\left(s_{j}^{i} \in S^{i}, i \in N\right),
$$


(2) $\quad \sigma_{j}^{i}=0, \quad\left(s_{j}^{i} \notin B^{i}, i \in N\right)$,

$$
\begin{aligned}
& \lambda_{j}^{i}=0, \quad\left(s_{j}^{i} \in B^{i}, i \in N\right), \\
& \sum_{s_{j}^{i} \in S^{i}} \sigma_{j}^{i}-1=0, \quad(i \in N), \\
& \sigma_{j}^{i} \geq 0, \quad\left(s_{j}^{i} \in B^{i}, i \in N\right), \\
& \lambda_{j}^{i} \geq 0, \quad\left(s_{j}^{i} \notin B^{i}, i \in N\right), \\
& t \geq 0, \\
& -t+1 \geq 0 .
\end{aligned}
$$

Theorem 3.1 implies that $(t, \sigma) \in \mathcal{L}\left(\Gamma, p, B^{*}\right)$ if and only if there exists $\lambda \in \mathbb{R}^{m^{*}}$ and $\mu \in \mathbb{R}^{n}$ such that the equalities (1)-(4) and the inequalities (5)-(8) are satisfied.

Theorem 3.1 Let a game $\Gamma \in \mathcal{G}$ and a prior $p \in \Sigma$ be given. For all admissible subsets $B^{*}$ of $S^{*}$, the sets $\mathcal{L}\left(\Gamma, p, B^{*}\right)$ and $\mathcal{O}\left(\Gamma, p, B^{*}\right)$ are $C^{\infty}$ diffeomorphic.

Proof Let $B^{*}$ be an admissible subset of $S^{*}$. For every $i \in N$ take an element $s_{j}^{i} \in B^{*}$. Define the function $f:[0,1] \times \Sigma \rightarrow \mathbb{R} \times \mathbb{R}^{m^{*}} \times \mathbb{R}^{m^{*}} \times \mathbb{R}^{n}$ by $f(t, \sigma)=(t, \sigma, \lambda$, $\mu)$, where $\lambda_{\ell}^{i}=v^{i}\left(t ; \sigma^{-i}, s_{j}^{i}\right)-v^{i}\left(t ; \sigma^{-i}, s_{\ell}^{i}\right)$ and $\mu^{i}=v^{i}\left(t ; \sigma^{-i}, s_{j}^{i}\right)$. Then $f(t, \sigma) \in \mathcal{O}\left(\Gamma, p, B^{*}\right)$ if and only if $(t, \sigma) \in \mathcal{L}\left(\Gamma, p, B^{*}\right)$. Note that $f$ defined in this way is a $C^{\infty}$ diffeomorphism.

In the proof of Theorem 3.1 it is shown that for all $(t, \sigma) \in \mathcal{L}\left(\Gamma, p, B^{*}\right)$ there is a unique $\lambda$ and a unique $\mu$ such that $(t, \sigma, \lambda, \mu) \in \mathcal{O}\left(\Gamma, p, B^{*}\right)$. Vice versa, for all $(t, \sigma, \lambda, \mu) \in \mathcal{O}\left(\Gamma, p, B^{*}\right)$ it holds that $(t, \sigma) \in \mathcal{L}\left(\Gamma, p, B^{*}\right)$.

The analysis of the system of equalities and inequalities (1)-(8) provides the following result.

Theorem 3.2 There exists an open set of games and priors $(\Gamma, p) \in \mathcal{G} \times \Sigma$ with full Lebesgue measure ${ }^{1}$ such that for all admissible subsets $B^{*}$ of $S^{*}$, the set $\mathcal{L}\left(\Gamma, p, B^{*}\right)$ is a compact 1dimensional $C^{\infty}$ manifold with boundary. Moreover, $(t, \sigma)$ is a boundary point of $\mathcal{L}\left(\Gamma, p, B^{*}\right)$ if and only if either $\sigma_{j}^{i}=0$ for exactly one $s_{j}^{i} \in B^{*}$, or exactly one $s_{j}^{i} \notin B^{*}$ is a best response to $\sigma^{-i}$, or $t=0$, or $t=1$.

Proof See Section 3.7 .

This theorem implies that, for almost every $\Gamma$ and $p$, for all admissible subsets $B^{*}$ of $S^{*}$ the set $\mathcal{L}\left(\Gamma, p, B^{*}\right)$ consists of a finite number of smooth arcs and loops (see for instance MasColell (1985), H.1.(vi)). The structure of $\mathcal{L}\left(\Gamma, p, B^{*}\right)$ is therefore a simple one; all kinds of complications like bifurcations, spirals, higher dimensional solution sets, diverging behavior, etc. are excluded. Theorem 3.2 does not claim that the set $\mathcal{L}\left(\Gamma, p, B^{*}\right)$ is non-empty. Since the empty set qualifies as a $k$-dimensional manifold for any $k$ by definition, a 1-dimensional manifold may be empty.

\footnotetext{
${ }^{1} \mathcal{G}$ is endowed with a measure in the standard way, for details see for instance van Damme (1987), page 25.
} 
To prove Theorem 3.2, it is first shown that, for generic $\Gamma$ and $p, \mathcal{O}\left(\Gamma, p, B^{*}\right)$ is a compact 1-dimensional $C^{\infty}$ manifold with boundary. By Theorem 3.1, this property carries over to $\mathcal{L}\left(\Gamma, p, B^{*}\right)$. Notice that the set $\mathcal{O}\left(\Gamma, p, B^{*}\right)$ is described in (1)-(8) as a system of equalities and inequalities with $2 m^{*}+n$ equations and $2 m^{*}+n+1$ unknowns. A 1-dimensional solution set is therefore what one expects.

The set $\mathcal{L}\left(\Gamma, p, B^{*}\right)$ consists of a finite number of arcs and loops. If it contains some arcs, it also has a boundary. The next result makes some very precise statements on the properties of boundary points.

Theorem 3.3 There exists an open set of games and priors $(\Gamma, p) \in \mathcal{G} \times \Sigma$ with full Lebesgue measure such that a boundary point $(t, \sigma)$ of $\mathcal{L}\left(\Gamma, p, B^{*}\right)$ is either

(i) not a boundary point of $\mathcal{L}\left(\Gamma, p, \bar{B}^{*}\right)$ for all $\bar{B}^{*} \neq B^{*}$ and lies in $\{0,1\} \times \Sigma$,

(ii) or, is a boundary point of exactly one $\mathcal{L}\left(\Gamma, p, \bar{B}^{*}\right)$ with $\bar{B}^{*} \neq B^{*}$ and belongs to $(0,1) \times \Sigma$. Moreover, $B^{*}$ and $\bar{B}^{*}$ differ in exactly one element, say $s_{j}^{i}$, for which $\sigma_{j}^{i}=0$ and $s_{j}^{i}$ is a best response to $\sigma^{-i}$ in $\Gamma^{t}$.

Proof By Theorem 3.2 it follows that in a boundary point of $\mathcal{L}\left(\Gamma, p, B^{*}\right)$ exactly one of the inequalities (5)-(8) is binding. If the binding inequality is (7) or (8) alternative (i) holds. If one of the inequalities from (5) or (6) is binding, the second alternative holds. More precisely, if an inequality from (5) is binding, it holds for exactly one $s_{j}^{i} \in B^{*}$ that $\sigma_{j}^{i}=0$ and the boundary point of $\mathcal{L}\left(\Gamma, p, B^{*}\right)$ is also a boundary point of $\mathcal{L}\left(\Gamma, p, \bar{B}^{*}\right)$, where $\bar{B}^{*}=B^{*} \backslash\left\{s_{j}^{i}\right\}$; if an inequality from (6) is binding, it holds that $\lambda_{j}^{i}=0$ for exactly one $s_{j}^{i} \notin B^{*}$ and the boundary point of $\mathcal{L}\left(\Gamma, p, B^{*}\right)$ is also a boundary point of $\mathcal{L}\left(\Gamma, p, \bar{B}^{*}\right)$, where $\bar{B}^{*}=B^{*} \cup\left\{s_{j}^{i}\right\}$.

If $\mathcal{L}\left(\Gamma, p, B^{*}\right)$ has a boundary point in $(0,1) \times \Sigma$, then there is a unique admissible subset $\bar{B}^{*}$ such that $\mathcal{L}\left(\Gamma, p, \bar{B}^{*}\right)$ has this boundary point in common with $\mathcal{L}\left(\Gamma, p, B^{*}\right)$. The cardinality of $\bar{B}^{*}$ is one less or one greater than the cardinality of $B^{*}$, depending on whether in the common boundary point a strategy in $B^{*}$ is played with probability zero, or whether a strategy not in $B^{*}$ is a best response. The uniqueness of the set $\bar{B}^{*}$ implies that the sets $\mathcal{L}\left(\Gamma, p, B^{*}\right)$ and $\mathcal{L}\left(\Gamma, p, \bar{B}^{*}\right)$ are nicely linked to each other. Generically, it does not happen that three sets have a common boundary point, nor does it happen that a boundary point of one set intersects the interior of another set. All this implies that $\mathcal{L}(\Gamma, p)$ does not display bifurcations.

If $\mathcal{L}\left(\Gamma, p, B^{*}\right)$ has a boundary point in $\{0,1\} \times \Sigma$, then this point does not belong to any other set $\mathcal{L}\left(\Gamma, p, \bar{B}^{*}\right)$. This implies that such a boundary point is also a boundary point of $\mathcal{L}(\Gamma, p)$.

Formally, the structure of $\mathcal{L}(\Gamma, p)$ is as follows.

Theorem 3.4 There exists an open set of games and priors $(\Gamma, p) \in \mathcal{G} \times \Sigma$ with full Lebesgue measure such that $\mathcal{L}(\Gamma, p)$ is a compact 1-dimensional piecewise $C^{\infty}$ manifold with boundary. 
The boundary of $\mathcal{L}(\Gamma, p)$ is given by the intersection of $\mathcal{L}(\Gamma, p)$ and $\{0,1\} \times \Sigma$. There is a unique boundary point in $\{0\} \times \Sigma$.

\section{Proof See Section 3.7.}

For almost every $\Gamma$ and $p$, the set $\mathcal{L}(\Gamma, p)$ consists of a finite number of arcs and loops. Although it is not necessarily the case that these arcs and loops are smooth, the number of nondifferentiabilities is finite at most. Theorem 3.3 implies that all arcs in $\mathcal{L}(\Gamma, p)$ start and end in $\{0,1\} \times \Sigma$. Each such path consists of a finite sequence of smooth $\operatorname{arcs}$ of the sets $\mathcal{L}\left(\Gamma, p, B^{*}\right)$. A loop in $\mathcal{L}(\Gamma, p)$ consists either of a finite sequence (at least two) of differentiable arcs in the sets $\mathcal{L}\left(\Gamma, p, B^{*}\right)$ or is a loop of one set $\mathcal{L}\left(\Gamma, p, B^{*}\right)$.

Generically, each player $i$ has a unique best response to the prior, so generically there is exactly one point of $\mathcal{L}(\Gamma, p)$ that belongs to $\{0\} \times \Sigma$. This point is both a corner point of $\{0\} \times \Sigma$ and a boundary point of $\mathcal{L}\left(\Gamma, p, B^{*}\right)$, where $B^{*}$ consists of the following $n$ strategies: for each player the best response to the prior. By Theorem 3.4 this point is the starting point of a uniquely defined arc of $\mathcal{L}(\Gamma, p)$. This arc is the unique feasible path of $\mathcal{L}(\Gamma, p)$ that transforms prior beliefs into equilibrium beliefs.

Theorem 3.5 There exists an open set of games and priors $(\Gamma, p) \in \mathcal{G} \times \Sigma$ with full Lebesgue measure such that the linear tracing procedure is well-defined.

By following the feasible path starting in the unique point $\mathcal{L}(\Gamma, p) \cap(\{0\} \cap \Sigma)$ a Nash equilibrium of the game $\Gamma$ is found. The set $\mathcal{L}(\Gamma, p) \cap(\{1\} \times \Sigma)$ consists of all equilibria of the game $\Gamma$. Precisely one of these equilibria is an element of the feasible path of $\mathcal{L}(\Gamma, p)$. Any other equilibrium is a boundary point of $\mathcal{L}(\Gamma, p)$ and is therefore part of some arc of $\mathcal{L}(\Gamma, p)$. A moment of reflection makes clear that the remaining equilibria are pairwise connected by arcs from $\mathcal{L}(\Gamma, p)$, and so the number of Nash equilibria is odd.

Theorem 3.6 There exists open set of games $\Gamma \in \mathcal{G}$ with full Lebesgue measure such that the number of Nash equilibria is odd.

Next an example follows in order to illustrate the theorems. Consider the game $\Gamma$ depicted in Figure 3.1 coming from Harsanyi and Selten (1988). In this game, player 1 is the row player having strategies $s_{1}^{1}$ and $s_{2}^{1}$. Player 2 is the column player having strategies $s_{1}^{2}$ and $s_{2}^{2}$. For each cell, the payoff to player 1 is displayed in the upper-left corner. The payoff to player 2 is displayed in the lower-right corner. So, when player 1 plays his first strategy $s_{1}^{1}$ and player 2 plays his first strategy $s_{1}^{2}$, player 1 obtains a payoff of 2 and player 2 obtains a payoff of 1 .

Take for the prior $p=\left(p^{1}, p^{2}\right)=\left(\left(\frac{1}{2}, \frac{1}{2}\right),\left(\frac{2}{3}, \frac{1}{3}\right)\right)$. Expecting that player 2 plays his first strategy with probability $2 / 3$ and his second strategy with probability $1 / 3$, by playing $s_{1}^{1}$ player 1 expects a payoff of $\frac{2}{3} \cdot 2+\frac{1}{3} \cdot 0=\frac{4}{3}$. By playing $s_{2}^{1}$, player 1 expects a payoff of $\frac{1}{3}$. Expecting 


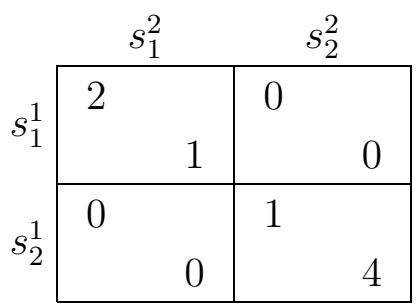

Figure 3.1: Game $\Gamma$.

that player 1 plays according to the prior, player 2 expects $\frac{1}{2}$ by playing $s_{1}^{2}$ and 2 by playing $s_{2}^{2}$. Clearly, this prior yields the game $\Gamma^{0}$ depicted in Figure 3.2 as our starting game.

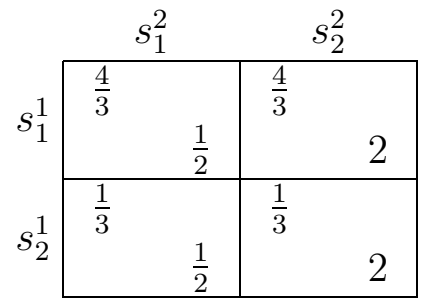

\begin{tabular}{|c|c|c|}
\hline & $s_{1}^{2}$ & $s_{2}^{2}$ \\
\hline & $\frac{4+2 t}{3}$ & $\frac{4-4 t}{3}$ \\
\hline & $\underline{1+t}$ & $2-2 t$ \\
\hline & $\frac{1-t}{3}$ & $\frac{1+2 t}{3}$ \\
\hline & $\frac{1-t}{2}$ & $2+2 t$ \\
\hline
\end{tabular}

Figure 3.2: Games $\Gamma^{0}$ and $\Gamma^{t}$.

The game $\Gamma^{t}$ of Figure 3.2 equals $(1-t) \cdot \Gamma^{0}+t \cdot \Gamma$. In Table 3.1 the set $\mathcal{L}\left(\Gamma, p, B^{*}\right)$ is described for all admissible sets $B^{*}$. Figure 3.3 shows the entire graph of the linear tracing procedure $\mathcal{L}(\Gamma, p)=\bigcup_{B^{*}} \mathcal{L}\left(\Gamma, p, B^{*}\right)$.

\begin{tabular}{ll}
\hline \multicolumn{1}{c}{$B^{*}$} & \multicolumn{1}{c}{$\mathcal{L}\left(\Gamma, p, B^{*}\right)$} \\
\hline$\left\{s_{1}^{1}, s_{1}^{2}\right\}$ & $\left\{\left(t,\left(s_{1}^{1}, s_{1}^{2}\right)\right) \mid t \in\left[\frac{3}{5}, 1\right]\right\}$ \\
$\left\{s_{1}^{1}, s_{2}^{2}\right\}$ & $\left\{\left(t,\left(s_{1}^{1}, s_{2}^{2}\right)\right) \mid t \in\left[0, \frac{1}{2}\right]\right\}$ \\
$\left\{s_{2}^{1}, s_{1}^{2}\right\}$ & $\emptyset$ \\
$\left\{s_{2}^{1}, s_{2}^{2}\right\}$ & $\left\{\left(t,\left(s_{2}^{1}, s_{2}^{2}\right)\right) \mid t \in\left[\frac{1}{2}, 1\right]\right\}$ \\
$\left\{s_{1}^{1}, s_{1}^{2}, s_{2}^{2}\right\}$ & $\left\{\left(\frac{3}{5},\left(s_{1}^{1},(r, 1-r)\right)\right) \mid r \in\left[\frac{1}{9}, 1\right]\right\}$ \\
$\left\{s_{2}^{1}, s_{1}^{2}, s_{2}^{2}\right\}$ & $\emptyset$ \\
$\left\{s_{1}^{1}, s_{2}^{1}, s_{1}^{2}\right\}$ & $\emptyset$ \\
$\left\{s_{1}^{1}, s_{2}^{1}, s_{2}^{2}\right\}$ & $\left\{\left(\frac{1}{2},\left((r, 1-r), s_{2}^{2}\right)\right) \mid r \in[0,1]\right\}$ \\
$\left\{s_{1}^{1}, s_{2}^{1}, s_{1}^{2}, s_{2}^{2}\right\}$ & $\left\{\left(t,\left(\left(\frac{5 t+3}{10 t}, \frac{5 t-3}{10 t}\right),\left(\frac{2 t-1}{3 t}, \frac{t+1}{3 t}\right)\right)\right) \mid t \in\left[\frac{3}{5}, 1\right]\right\}$ \\
\hline
\end{tabular}

Table 3.1: Description of the sets $\mathcal{L}\left(\Gamma, p, B^{*}\right)$. 


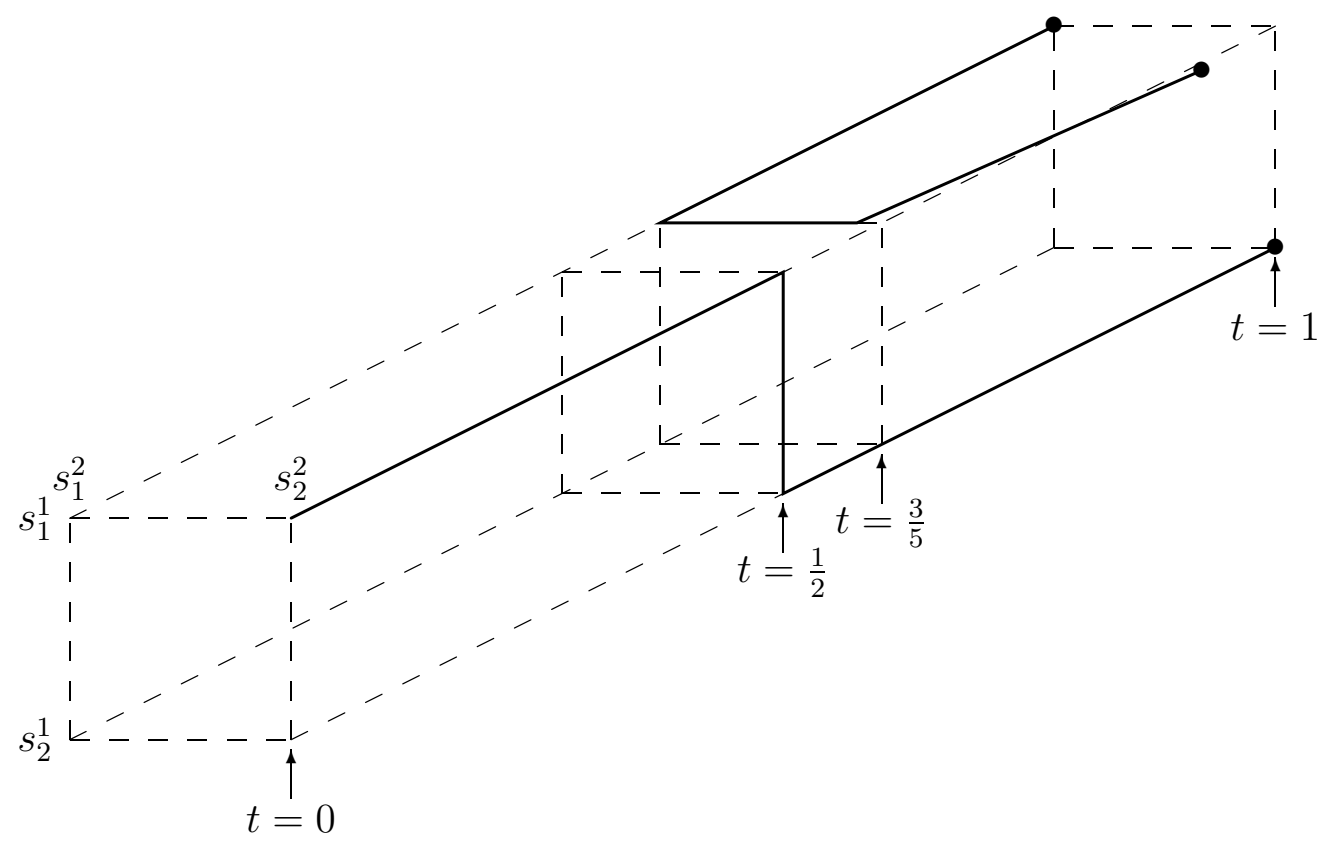

Figure 3.3: The linear tracing procedure.

The feasible path of the linear tracing procedure starts in the point $\left(0,\left(s_{1}^{1}, s_{2}^{2}\right)\right)$ with $B^{*}=$ $\left\{s_{1}^{1}, s_{2}^{2}\right\}$. As long as $t<\frac{1}{2}$, the equalities (1)-(4) determine the feasible path. At $t=\frac{1}{2}$ inequality (6) belonging to strategy $s_{2}^{1}$ is binding, which implies that the second pure strategy of player 1 is a best response to a belief of player 1 that puts probability $1 / 2$ on the prior and probability $1 / 2$ on strategy $s_{2}^{2}$. A further continuation of the path determined by the equalities (1)-(4) without changing the set $B^{*}$ would make the value of $\lambda_{2}^{1}$ negative and thereby violates inequality (6). The set $B^{*}$ has to be expanded by including strategy $s_{2}^{1}$. Our new $B^{*}$ becomes $\left\{s_{1}^{1}, s_{2}^{1}, s_{2}^{2}\right\}$. Continue following the feasible path from $\left(\frac{1}{2},\left(s_{1}^{1}, s_{2}^{2}\right)\right)$, leads to the point $\left(\frac{1}{2},\left(s_{2}^{1}, s_{2}^{2}\right)\right)$. Continuing with the current $B^{*}$ violates inequality (5), since $\sigma_{1}^{1}$ becomes negative. Strategy $s_{1}^{1}$ has to be removed from $B^{*}$ and the new $B^{*}$ becomes $\left\{s_{2}^{1}, s_{2}^{2}\right\}$. The equalities (1)-(4) related to this $B^{*}$ determine the remainder of the path leading to a Nash equilibrium of the game.

The observations made so far suggest the following algorithm for the computation of the Nash equilibrium selected by the tracing procedure in $n$-person games. Define the admissible set $B^{*}$ that contains for each player $i$ the best response to the prior and start with a point $(0, \sigma)$ in $\mathcal{L}\left(\Gamma, p, B^{*}\right)$ such that $\sigma^{i}$ is a best response of player $i$ to the prior. Theorem 3.4 implies that $B^{*}$ and $\sigma$ are uniquely determined. The equalities (1)-(4) belonging to this $B^{*}$ determine at least a part of the feasible path. As long as the inequalities (5)-(8) hold with strict inequality the set $B^{*}$ stays unchanged. As soon as one of the inequalities from (5) or (6) gets binding, the set $B^{*}$ needs to be changed. When the binding inequality belongs to (5), say $\sigma_{j}^{i}=0$ while $s_{j}^{i} \in B^{*}$, 
strategy $s_{j}^{i}$ has to be removed from $B^{*}$. Obviously, this cannot happen for the set $B^{*}$ obtained at the start. If the binding inequality belongs to (6), say $\lambda_{j}^{i}=0$ while $s_{j}^{i} \notin B^{*}$, strategy $s_{j}^{i}$ has to be added to $B^{*}$. In both situations there is a strategy $s_{j}^{i}$ for which $\sigma_{j}^{i}=0$ and $\lambda_{j}^{i}=0$. In general this leads to a kink in the feasible path of the linear tracing procedure. This procedure is repeated with our new $B^{*}$. Note that inequality (7) is only binding in the starting point. When inequality (8) is binding, a Nash equilibrium is found.

In Figure 3.3 it can be seen that the linear tracing procedure determines a whole range of equilibria at $t=\frac{1}{2}$. In general, it is even possible that the linear tracing procedure buckles and moves backwards in $t$ (see Section 4.19 of Harsanyi and Selten (1988) for an explicit example).

\subsection{A differentiable approach}

The previous section presents a method that can be used for the computation of a Nash equilibrium, in particular the Nash equilibrium selected by the Harsanyi-Selten theory. A potential drawback of that method is that one has to check continuously whether the system of equalities used is still appropriate, and if not, one has to switch between different systems of equalities.

The idea of switching homotopies has only been used in the literature recently. In the context of general equilibrium theory with incomplete markets switching homotopies are used by Brown, DeMarzo and Eaves (1996). In the present set-up, switching homotopies can be a serious problem in terms of computing time. There are $\prod_{i \in N}\left(2^{m^{i}}-1\right)$ different sets $B^{*}$, whereas each one of them may be visited several times in the course of the algorithm.

In the remaining of this section it is shown that for the problem of computing Nash equilibria, switching homotopies can be avoided. It is possible to formulate one, everywhere differentiable, homotopy. To obtain differentiability a well-chosen transformation of variables is used, which is also used in Garcia and Zangwill (1981), and Herings and Schmedders (2001). Define for all $\alpha \in \mathbb{R}^{m^{*}}$

$$
\sigma_{j}^{i}(\alpha)=\left[\max \left\{0, \alpha_{j}^{i}\right\}\right]^{2} \quad \text { and } \quad \lambda_{j}^{i}(\alpha)=\left[\max \left\{0,-\alpha_{j}^{i}\right\}\right]^{2}
$$

After this transformation of variables, the Kuhn-Tucker first-order conditions that provide necessary and sufficient conditions for $\sigma^{i}$ to be a best response against $\bar{\sigma}^{-i}$ in the game $\Gamma^{\bar{t}}$ become

$$
\begin{aligned}
& v^{i}\left(\bar{t} ; \bar{\sigma}^{-i}(\alpha), s_{j}^{i}\right)+\lambda_{j}^{i}(\alpha)-\mu^{i}=0, \quad\left(s_{j}^{i} \in S^{i}, i \in N\right), \\
& \lambda_{j}^{i}(\alpha) \geq 0, \quad \sigma_{j}^{i}(\alpha) \geq 0, \quad \lambda_{j}^{i}(\alpha) \sigma_{j}^{i}(\alpha)=0, \quad\left(s^{i} \in S^{i}, i \in N\right) \\
& \sum_{s_{j}^{i} \in S^{i}} \sigma_{j}^{i}(\alpha)-1=0, \quad(i \in N) .
\end{aligned}
$$

Owing to the proposed transformation of variables, the conditions $\lambda_{j}^{i}(\alpha) \geq 0, \sigma_{j}^{i}(\alpha) \geq 0$ and $\lambda_{j}^{i}(\alpha) \sigma_{j}^{i}(\alpha)=0$ are trivially satisfied. The (in)equalities in (1)-(8) that characterize the set 
$\mathcal{O}\left(\Gamma, p, B^{*}\right)$ correspond to solutions $(t, \alpha, \mu) \in \mathbb{R} \times \mathbb{R}^{m^{*}} \times \mathbb{R}^{n}$ with $\alpha_{j}^{i} \geq 0$ if $s_{j}^{i} \in B^{*}$ and $\alpha_{j}^{i} \leq 0$ if $s_{j}^{i} \notin B^{*}$ such that
(a) $\quad v^{i}\left(t ; \sigma^{-i}(\alpha), s_{j}^{i}\right)+\lambda_{j}^{i}(\alpha)-\mu^{i}=0, \quad\left(s^{i} \in S^{i}, i \in N\right)$,
(b) $\quad \sum_{s_{j}^{i} \in S^{i}} \sigma_{j}^{i}(\alpha)-1=0, \quad(i \in N)$,
(c) $\quad t \geq 0$,
(d) $\quad-t+1 \geq 0$.

Looking at the system of equalities and inequalities (a)-(d) it is striking that the set $B^{*}$ has disappeared. The same system of equalities and inequalities can be used, irrespective of the set $B^{*}$. The role of $B^{*}$ is taken over by the vector $\alpha$; more precisely, by the sign-combinations of the components of $\alpha$. Actually, given a point $(t, \alpha, \mu)$ satisfying the (in)equalities (a)-(d), a set $B^{*}$ such that $(t, \sigma(\alpha), \lambda(\alpha), \mu)$ satisfies the (in)equalities (1)-(8) for this particular $B^{*}$ is easily reconstructed. More precisely, such a set $B^{*}$ consists of all strategies $s_{j}^{i}$ for which $\alpha_{j}^{i}$ is positive and does not contain strategies $s_{j}^{i}$ for which $\alpha_{j}^{i}$ is negative. Admissibility is trivially satisfied since by (b) it follows that for each player $i$ there is at least one strategy $s_{j}^{i}$ such that $\alpha_{j}^{i}$ is positive. Figure 3.4 illustrates the relation between the vector $\alpha$ and the set $B^{*}$.
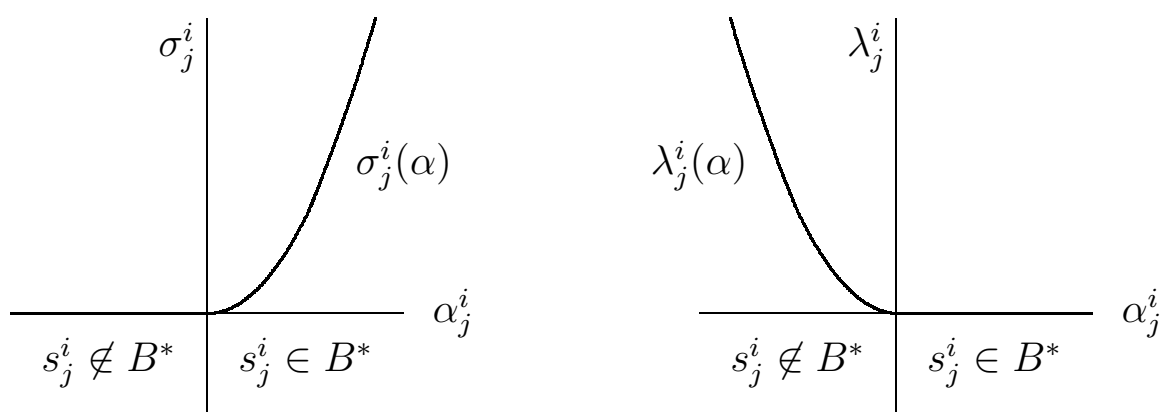

Figure 3.4: Relation between $\sigma_{j}^{i}$ and $\alpha_{j}^{i}$ and between $\lambda_{j}^{i}$ and $\alpha_{j}^{i}$.

Counting equations and unknowns in the system (a)-(d) shows that there is one degree of freedom, and therefore one expects a 1-dimensional solution set. Consider any solution $(t, \alpha, \mu)$ to (a)-(d). When $\alpha_{j}^{i}=0$, then both $\sigma_{j}^{i}(\alpha)$ and $\lambda_{j}^{i}(\alpha)$ are zero. This implies that there are exactly two admissible subsets of $S^{*}$ for which the set of (in)equalities (1)-(8) are satisfied. If along a solution curve $\alpha_{j}^{i}$ is increasing while passing zero, then $\sigma_{j}^{i}(\alpha)$ gets positive and $B_{\text {new }}^{*}=B_{\text {old }}^{*} \cup$ $\left\{s_{j}^{i}\right\}$. If $\alpha_{j}^{i}$ is decreasing while passing zero, then $\lambda_{j}^{i}(\alpha)$ gets positive and $B_{\text {new }}^{*}=B_{\text {old }}^{*} \backslash\left\{s_{j}^{i}\right\}$. Whenever $\alpha_{j}^{i}$ passes zero, a kink occurs in $\mathcal{L}(\Gamma, p)$.

The left-hand sides of the equalities (a)-(b) specify the homotopy $\mathcal{H}:[0,1] \times \mathbb{R}^{m^{*}} \times \mathbb{R}^{n} \rightarrow$ 


$$
\begin{aligned}
& \mathbb{R}^{m^{*}} \times \mathbb{R}^{n}, \\
& \mathcal{H}(t, \alpha, \mu)=\left(\begin{array}{cc}
v^{i}\left(t ; \sigma^{-i}(\alpha), s_{j}^{i}\right)+\lambda_{j}^{i}(\alpha)-\mu^{i} & \left(s_{j}^{i} \in S^{i}, i \in N\right) \\
\sum_{s_{j}^{i} \in S^{i}} \sigma_{j}^{i}(\alpha)-1 & (i \in N)
\end{array}\right) .
\end{aligned}
$$

The homotopy function $\mathcal{H}$ is continuously differentiable. ${ }^{2}$ The inequalities (c) and (d) are satisfied as the homotopy takes $[0,1]$ as the domain for the variable $t$. It has the salient feature that its zeros describe the linear tracing procedure, $(t, \alpha, \mu) \in \mathcal{H}^{-1}(\{0\}) \Leftrightarrow(t, \sigma(\alpha)) \in \mathcal{L}(\Gamma, p)$. Starting at the unique point $\left(0, \alpha^{0}, \mu^{0}\right) \in \mathcal{H}^{-1}(\{0\})$ at $t=0$ and following the path described by the zeros of $\mathcal{H}$, we end up in a point $(1, \tilde{\alpha}, \tilde{\mu}) \in \mathcal{H}^{-1}(\{0\})$. This point generates the Nash equilibrium $\sigma(\tilde{\alpha})$ of $\Gamma$ selected by the Harsanyi-Selten theory.

Theorem 3.7 specifies the structure of the set of solutions to the homotopy, $\mathcal{H}^{-1}(\{0\})$.

Theorem 3.7 There exists an open set of games and priors $(\Gamma, p) \in \mathcal{G} \times \Sigma$ with full Lebesgue measure such that $\mathcal{H}^{-1}(\{0\})$ is a compact 1-dimensional $C^{1}$ manifold with boundary. The boundary of $\mathcal{H}^{-1}(\{0\})$ equals the intersection of $\mathcal{H}^{-1}(\{0\})$ and $\{0,1\} \times \mathbb{R}^{m^{*}} \times \mathbb{R}^{n}$. There is a unique boundary point in $\{0\} \times \mathbb{R}^{m^{*}} \times \mathbb{R}^{n}$.

\section{Proof See Section 3.7 .}

The set $\mathcal{H}^{-1}(\{0\})$ consists of finitely many differentiable arcs and loops. All arcs start and end in $\{0,1\} \times \mathbb{R}^{m^{*}} \times \mathbb{R}^{n}$. Loops have no points in common with $\{0,1\} \times \mathbb{R}^{m^{*}} \times \mathbb{R}^{n}$. There is exactly one arc that starts in $\{0\} \times \mathbb{R}^{m^{*}} \times \mathbb{R}^{n}$ and that ends in $\{1\} \times \mathbb{R}^{m^{*}} \times \mathbb{R}^{n}$ with a point $(1, \tilde{\alpha}, \tilde{\mu})$ that generates the Nash equilibrium selected by the tracing procedure. This arc is a transformation of the feasible path of the tracing procedure. All other arcs start and end in $\{1\} \times \mathbb{R}^{m^{*}} \times \mathbb{R}^{n}$ and connect two points inducing Nash equilibria of $\Gamma$.

The structure of $\mathcal{H}^{-1}(\{0\})$ is even simpler than the one of $\mathcal{L}(\Gamma, p)$. Not only, like for $\mathcal{L}(\Gamma, p)$, are complications like bifurcations, spirals, higher dimensional solutions sets, diverging behavior, etc. excluded. The arcs and loops in $\mathcal{H}^{-1}(\{0\})$ are differentiable everywhere. It is the transformation of variables that smoothes out the kinks. As a direct consequence, it is possible to calculate the derivative at each point of the feasible path, which makes it possible to follow the path by means of differentiable as opposed to simplicial methods.

It is very convenient to describe the variables $t, \sigma, \lambda, \alpha$ and $\mu$ as functions of pathlength $\tau$. By doing so, the feasible path of the linear tracing procedure of Figure 3.3 is as shown in Figure 3.5. The plot in panel (a) of Figure 3.5 shows $t, \sigma$ and $\lambda$ as a function of pathlength. To make the picture more clear, only informative values of the variables are depicted. In particular this means that for each pure strategy $s_{j}^{i}$, either $\sigma_{j}^{i}$ or $-\lambda_{j}^{i}$ is plotted, depending on which one is non-zero.

\footnotetext{
${ }^{2}$ When for the transformation a power of $1+\xi$ would have been used, the homotopy-function would be $r$ times continuously differentiable, where $r=\lfloor\xi\rfloor$, that is, the largest integer smaller than $\xi$.
} 


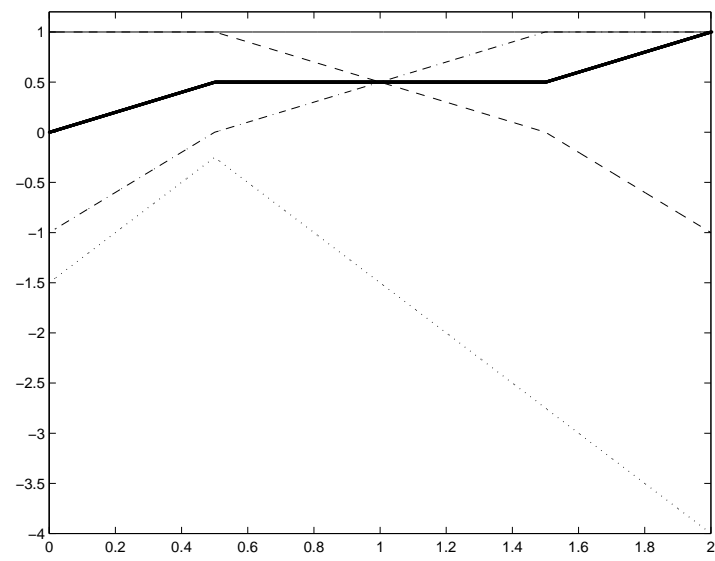

(a) Before transformation.

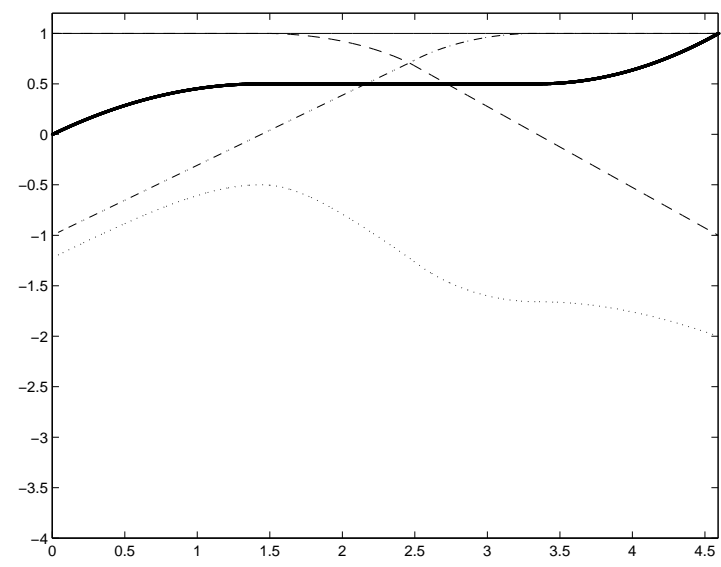

(b) After transformation.
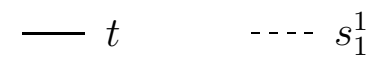

$s_{1}^{2}$

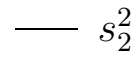

Figure 3.5: Homotopy-variables of the linear tracing procedure as a function of pathlength $\tau$.

The plot in panel (b) of Figure 3.5 shows the values of $t$ and $\alpha$ as a function of pathlength and therefore corresponds to the feasible path of the tracing procedure after the transformation.

The first plot shows that kinks appear for all variables when $s_{1}^{1}$ and $s_{2}^{1}$ are passing zero. In the second plot no kinks appear at all. In that plot, when $s_{1}^{1}$ or $s_{2}^{1}$ passes zero, the derivatives of all variables with respect to pathlength are equal to zero, except the variable passing zero. This is a general phenomenon and follows from the proofs in Section 3.7.

\subsection{Implementation}

This section is devoted to the implementation of the homotopy function. Because of differentiability of the homotopy path, it is possible to apply standard numerical path-following techniques that are available in professionally programmed software.

What is programmed are a number of FORTRAN-subroutines belonging to the softwarepackage HOMPACK, a FORTRAN77 program (see Watson, Billups and Morgan (1987)). ${ }^{3}$ HoMPACK provides three qualitatively different algorithms for tracking the zero curve of the homotopy: ordinary differential equation-based, normal flow, and augmented Jacobian matrix. Separate routines are also provided for dense and sparse matrices. The algorithm used for the implementation is FIXPDF, which is an ordinary differential equation-based algorithm working with dense Jacobian matrices. FIXPDF is chosen because it is more robust than the other algorithms

\footnotetext{
${ }^{3}$ http://Www.netlib.org/hompack/index.html
} 
even though this may come at the cost of higher computing times.

The homotopy path is parameterized by pathlength $\tau$. Thus $t=t(\tau), \alpha=\alpha(\tau)$ and $\mu=\mu(\tau)$ along the homotopy path, and $\mathcal{H}(t(\tau), \alpha(\tau), \mu(\tau))=0$ identically in $\tau$. The differential equation is characterized by

$$
\begin{aligned}
& \frac{\mathrm{d}}{\mathrm{d} \tau} \mathcal{H}(t(\tau), \alpha(\tau), \mu(\tau))=\partial \mathcal{H}(t(\tau), \alpha(\tau), \mu(\tau)) \cdot\left(\begin{array}{c}
\mathrm{d} t / \mathrm{d} \tau \\
\mathrm{d} \alpha / \mathrm{d} \tau \\
\mathrm{d} \mu / \mathrm{d} \tau
\end{array}\right)=0, \\
& \left\|\left(\frac{\mathrm{d} t}{\mathrm{~d} \tau}, \frac{\mathrm{d} \alpha}{\mathrm{d} \tau}, \frac{\mathrm{d} \mu}{\mathrm{d} \tau}\right)\right\|_{2}=1
\end{aligned}
$$

and initial conditions given by

$$
(t(0), \alpha(0), \mu(0))=\mathcal{H}^{-1}(\{0\}) \cap\left(\{0\} \times \mathbb{R}^{m^{*}} \times \mathbb{R}^{n}\right) .
$$

The homotopy path corresponds to the trajectory of the initial value problem. When $t(\bar{\tau})=1$, $(\alpha(\bar{\tau}), \mu(\bar{\tau}))$ is a zeropoint of $\mathcal{H}$ and $\sigma(\alpha(\bar{\tau}))$ is a Nash equilibrium of game $\Gamma$ with equilibrium payoff $\mu(\bar{\tau})$. Details on how to solve the initial value problem can be found in Watson (1979) and Watson and Fenner (1980).

The homotopy function that is implemented is the one as described in Section 3.4. It may be possible to improve on computing times by using a transformation with a power less than 2 , or by rescaling utilities and probabilities. Moreover, HOMPACK provides the possibility to adjust a number of parameters, mainly related to the accuracy by which the homotopy path is followed. An extensive digress on the optimal numerical implementation of the homotopy is beyond the scope of the current analysis.

Among others the algorithm is applied to the example of Harsanyi and Selten from the previous sections. Figure 3.6 shows five plots. The first plot corresponds to the homotopy-variable $t$ and is plotted with respect to pathlength $\tau$. Each of the other four plots correspond to the homotopy-variables $\alpha_{1}^{1}, \alpha_{2}^{1}, \alpha_{1}^{2}$, and $\alpha_{2}^{2}$. These variables are also plotted as a function of pathlength. For all five plots, the dotted lines show the analytical solution path and the solid lines show the numerical solution paths.

For a number of pairs $(n, m)$, where $n$ is the number of players and $m$ the common number of pure strategies they have, five games are generated randomly. All payoffs are chosen independently from one another out of the uniform distribution on $[0,1]$. The prior is chosen such that the players play all their strategies with equal probability. Table 3.2 shows the mean time in seconds (the upper number) and the mean number of function evaluations (the lower number) needed to compute a Nash equilibrium. Furthermore, between brackets, the standard deviations are given. $^{4}$

\footnotetext{
${ }^{4}$ The computations were performed on a PC operating under Windows 98 with an Intel Pentium II $350 \mathrm{MHz}$ processor and $64 \mathrm{Mb}$ workspace.
} 


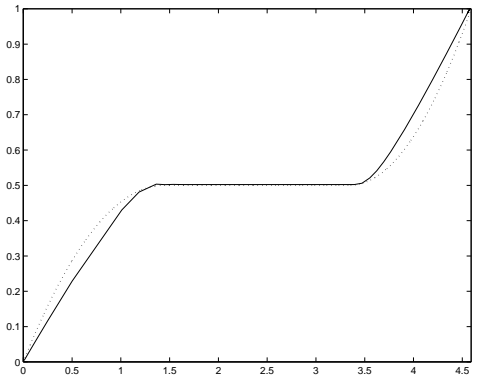

The variable $t$.

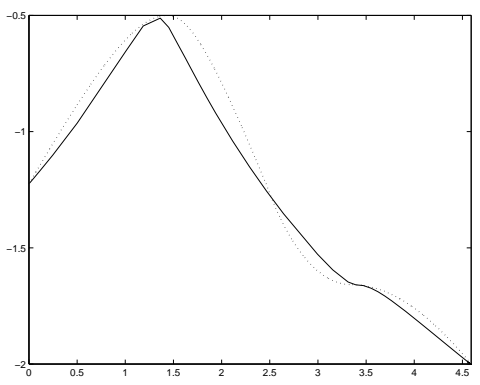

The variable $\alpha_{1}^{2}$.

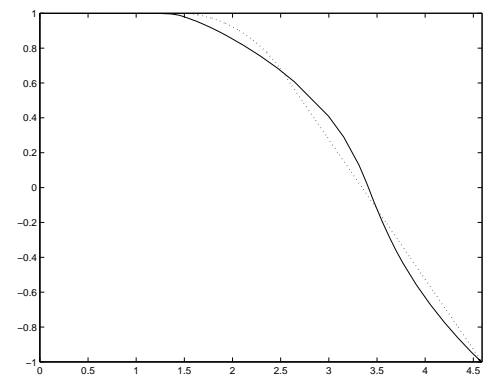

The variable $\alpha_{1}^{1}$.

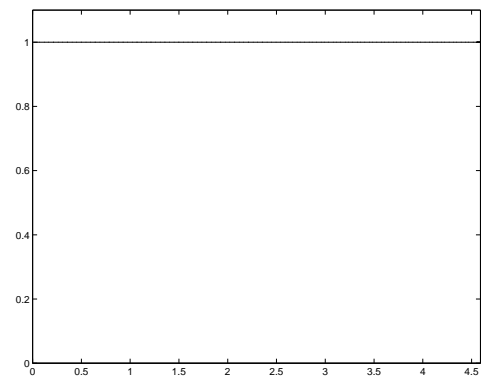

The variable $\alpha_{2}^{2}$.

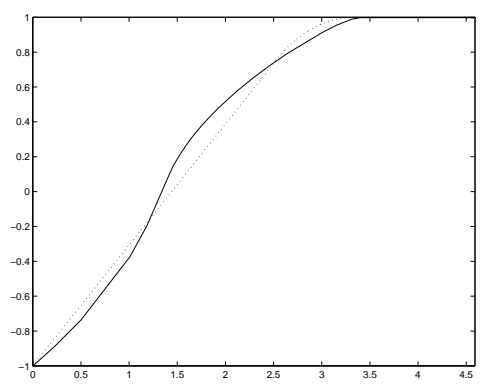

The variable $\alpha_{2}^{1}$.

Figure 3.6: The behavior of the homotopy-variables along the zero curve as a function of pathlength $\tau$.

The table shows that computation times were less than one minute for most cases analyzed. The standard deviation of computation times is fairly large. This can be explained by the fact that sometimes the best response to the prior is a Nash equilibrium, in which case the homotopy path is a straight line from $t=0$ to $t=1$. In other cases, none of the strategies played with positive probability against the prior is played with positive probability in a Nash equilibrium. The resulting homotopy path is curly and takes a long time to be tracked.

The table clearly conveys the impression that the mean computation time and the mean number of function evaluations is greater when a large number of agents each have a small number of pure strategies than when a small number of agents each have many pure strategies (in the table horizontally the numbers increase faster than vertically).

The maximal inaccuracy of our calculation amounts to $10^{-4}$, which means that the 2-norm of the value of the homotopy function is less than $10^{-4}$ in the computed equilibrium. From the specification of the homotopy function, this implies that the gain in payoff from a unilateral deviation by a player is at most $10^{-4}$.

For the same pairs $(n, m)$, Table 3.3 shows an estimation of the mean number of Nash equilibria with between brackets the standard error of the estimation (see Table 9 of McLennan (1999)). 


\begin{tabular}{ccccc}
\hline$m \backslash n$ & 2 & 3 & 4 & 5 \\
\hline 2 & $0.28(0.13)$ & $0.40(0.05)$ & $0.79(0.19)$ & $2.28(1.15)$ \\
& $44.20(23.79)$ & $143.60(20.94)$ & $136.40(40.92)$ & $178.40(111.69)$ \\
3 & $0.33(0.09)$ & $1.57(0.56)$ & $7.48(4.29)$ & $44.87(12.10)$ \\
& $127.40(33.70)$ & $272.60(156.79)$ & $355.40(227.83)$ & $455.20(122.19)$ \\
4 & $0.51(0.32)$ & $2.73(0.61)$ & $104.94(108.22)$ & $519.44(332.60)$ \\
& $151.60(152.50)$ & $197.40(54.04)$ & $1036.80(945.71)$ & $824.40(381.34)$ \\
5 & $0.98(0.31)$ & $16.89(12.02)$ & $360.16(250.91)$ \\
& $233.20(98.51)$ & $589.20(362.91)$ & $1215.40(730.73)$ & \\
6 & $1.14(0.35)$ & $24.84(25.97)$ & $442.47(147.78)$ & \\
& $182.00(64.39)$ & $461.80(481.87)$ & $686.00(201.86)$ & \\
7 & $2.51(1.39)$ & $51.59(43.43)$ & & \\
& $339.60(207.47)$ & $520.60(396.45)$ & & \\
8 & $3.97(1.74)$ & $179.90(162.42)$ & & \\
& $408.60(183.76)$ & $941.40(788.42)$ & & \\
9 & $4.37(3.27)$ & & & \\
5 & $345.20(276.51)$ & & &
\end{tabular}

Table 3.2: Average computation times and average number of function evaluations.

The numbers in the table suggests that multiplicity of Nash equilibria is a serious problem in game theory and that the specific method used for equilibrium selection is of crucial importance.

\begin{tabular}{ccccc}
\hline$m \backslash n$ & 2 & 3 & 4 & 5 \\
\hline 2 & $1.31(0.13)$ & $2.15(0.20)$ & $4.49(0.40)$ & $6.98(0.43)$ \\
3 & $1.52(0.12)$ & $3.76(0.33)$ & $18.01(1.22)$ & $81.82(3.32)$ \\
4 & $1.77(0.18)$ & $12.66(1.13)$ & $82.49(3.87)$ & $879.24(32.94)$ \\
5 & $2.64(0.44)$ & $27.23(2.78)$ & $440.02(18.86)$ & \\
6 & $2.61(0.52)$ & $65.69(4.19)$ & $2036.54(65.98)$ & \\
7 & $4.62(0.41)$ & $151.80(3.88)$ & & \\
8 & $5.52(0.37)$ & $355.63(8.60)$ & & \\
9 & $6.74(0.44)$ & & & \\
10 & $8.55(0.54)$ & & & \\
\hline
\end{tabular}

Table 3.3: Average number of equilibria.

The algorithm also provides the possibility to investigate how sensitive the Nash equilibrium selected by the linear tracing procedure is with respect to the choice of the prior. For the same pairs as before, five such games are randomly generated and for each game a Nash equilibrium 
is computed for five randomly generated priors. Table 3.4 shows the average number of different Nash equilibria found, with the standard deviation given between brackets.

\begin{tabular}{ccccc}
\hline$m \backslash n$ & 2 & 3 & 4 & 5 \\
\hline 2 & $1.00(0.00)$ & $1.20(0.45)$ & $1.40(0.55)$ & $1.40(0.89)$ \\
3 & $1.00(0.00)$ & $2.20(0.84)$ & $2.60(1.14)$ & $3.80(0.84)$ \\
4 & $1.20(0.45)$ & $2.40(0.89)$ & $4.00(0.71)$ & $4.60(0.89)$ \\
5 & $1.40(0.55)$ & $2.20(1.10)$ & $4.00(1.41)$ & \\
6 & $1.20(0.45)$ & $2.40(0.89)$ & $4.80(0.45)$ & \\
7 & $1.80(0.45)$ & $3.40(0.55)$ & & \\
8 & $1.60(0.89)$ & $2.80(1.48)$ & & \\
9 & $1.40(0.89)$ & & & \\
10 & $2.00(1.00)$ & & & \\
\cline { 1 - 2 }
\end{tabular}

Table 3.4: Multiplicity by variation of prior.

Since five different priors are tried, the maximal number of different Nash equilibria that can be found in this set-up is five. Notice that for cases with 4 or 5 players, each having at least 4 pure strategies, the average number found in our computations is above four, so close to the maximum possible. When the number of players is 2 , the average number of different Nash equilibria found is less than two, so close to the minimum possible. This confirms again that games with many players are far more difficult in all respects than games with few players. It also shows that multiplicity of Nash equilibria is a serious problem, and that the equilibrium selection method used is likely to be crucial.

\subsection{Summary}

In this chapter it is shown that generically the linear tracing procedure consists of a finite number of piecewise smooth arcs and loops. From this structure it is concluded that the linear tracing procedure is well-defined for almost all normal form games. An everywhere differentiable homotopy function is defined, whose zeros correspond one-to-one to elements of the linear tracing procedure. From this it is proved that the set of zeros of the homotopy function consists of a finite number of smooth arcs and loops. Finally, this homotopy function is used to compute the equilibrium selected by the tracing procedure numerically. The unique smooth feasible path is followed using a mathematical software package, which is able to follow smooth homotopy-paths numerically. In this way, the first globally convergent algorithm that exploits the differentiability present in normal form games is constructed. The numerical performance of the algorithm is analyzed extensively. Further, it is showed that multiple equilibria can be found by varying the prior expectations. 


\subsection{Proofs}

Fix the number of players, the number of pure strategies for each player, and fix a prior $p \in \Sigma$. For any tuple of utility functions $u$ and any admissible subset $B^{*}$, define the functions $g^{B^{*}, u}$ : $\mathbb{R}^{1+2 m^{*}+n} \rightarrow \mathbb{R}^{2 m^{*}+n}$ and $h^{B^{*}, u}: \mathbb{R}^{1+2 m^{*}+n} \rightarrow \mathbb{R}^{m^{*}+2}$ in such a way that $g^{B^{*}, u}$ equals the lefthand side of the equalities (1)-(4) and $h^{B^{*}, u}$ the left-hand side of the inequalities (5)-(8),

$$
g^{B^{*}, u}(t, \sigma, \lambda, \mu)=\left(\begin{array}{ccc}
v^{i}\left(t ; \sigma^{-i}, s_{j}^{i}\right)+\lambda_{j}^{i}-\mu^{i} & \left(s_{j}^{i} \in S^{i}, i \in N\right) \\
\sigma_{j}^{i} & \left(s_{j}^{i} \notin B^{i}, i \in N\right) \\
\lambda_{j}^{i} & \left(s_{j}^{i} \in B^{i}, i \in N\right) \\
\sum_{s_{j}^{i} \in S^{i}} \sigma_{j}^{i}-1 & (i \in N)
\end{array}\right)
$$

and

$$
h^{B^{*}, u}(t, \sigma, \lambda, \mu)=\left(\begin{array}{cc}
\sigma_{j}^{i} & \left(s_{j}^{i} \in B^{i}, i \in N\right) \\
\lambda_{j}^{i} & \left(s_{j}^{i} \notin B^{i}, i \in N\right) \\
& t \\
& -t+1
\end{array}\right) .
$$

A point $(t, \sigma, \lambda, \mu)$ is a solution of the system of equations and inequalities (1)-(8) if and only if $g^{B^{*}, u}(t, \sigma, \lambda, \mu)=0$ and $h^{B^{*}, u}(t, \sigma, \lambda, \mu) \geq 0$.

Once the number of players and the number of pure strategies of each player are fixed, a normal form game is completely determined by the tuple of utility functions $u$, which can be represented by a vector in $\mathbb{R}^{m n}$. The standard topology and measure on $\mathbb{R}^{m n}$ therefore induce a topology and a measure on games. For every $u \in \mathbb{R}^{m n}$, we define the functions $g^{B^{*}}: \mathbb{R}^{1+2 m^{*}+n} \times$ $\mathbb{R}^{n m} \rightarrow \mathbb{R}^{2 m^{*}+n}$ and $h^{B^{*}}: \mathbb{R}^{1+2 m^{*}+n} \times \mathbb{R}^{n m} \rightarrow \mathbb{R}^{m^{*}+2}$ by $g^{B^{*}}(t, \sigma, \lambda, \mu, u)=g^{B^{*}, u}(t, \sigma, \lambda, \mu)$ and $h^{B^{*}}(t, \sigma, \lambda, \mu, u)=h^{B^{*}, u}(t, \sigma, \lambda, \mu)$. Figure 3.7 presents the matrix of derivatives of the functions $g^{B^{*}}$ and $h^{B^{*}}$ with respect to all variables, where

$$
\begin{aligned}
& E\left(i, s_{j^{\prime}}^{i^{\prime}}\right)= \begin{cases}1 & \text { if } i=i^{\prime} \\
0 & \text { otherwise }\end{cases} \\
& \partial_{u^{k}\left(s^{-k}, s_{\ell}^{k}\right)} v^{i}\left(t ; \sigma^{-i}, s_{j}^{i}\right)=0 \text { if } s_{j}^{i} \neq s_{\ell}^{k} \quad \text { for all } s^{-k} \in S^{-k},
\end{aligned}
$$

and

$$
\sum_{s^{-i} \in S^{-i}} \partial_{u^{i}\left(s^{-i}, s_{j}^{i}\right)} v^{i}\left(t ; \sigma^{-i}, s_{j}^{i}\right)=1 .
$$

Each row in Figure 3.7 corresponds to one of the equalities and inequalities in (1)-(8). To make the figure more clear, derivatives with respect to $\sigma$ are first taken for $s_{j}^{i} \in B^{*}$. The first applies to the derivatives with respect to $\lambda$ and the ordering of the equalities in (1). From the properties of the matrix, it follows that the matrices $\partial_{u} v$ and $E$ have full row rank. 


\begin{tabular}{|c|c|c|c|c|c|c|}
\hline & $\partial_{t}$ & $\begin{array}{c}\partial_{\sigma} \\
B^{*} \quad S^{*} \backslash B^{*}\end{array}$ & $\partial_{B^{*}} \partial_{S^{*} \backslash B^{*}}$ & $\partial_{\mu}$ & $\partial_{u}$ & \\
\hline (1) $m^{*}$ & $\partial_{t} v(\cdot)$ & $-\partial_{\sigma} v(\cdot)-$ & \begin{tabular}{c:c}
$I$ & 0 \\
\hdashline 0 & $I$
\end{tabular} & $-E^{\top}$ & $\partial_{u} v(\cdot)$ & $\begin{array}{l}B^{*} \\
- \\
S^{*} \backslash B^{*}\end{array}$ \\
\hline (2) & 0 & $0: I$ & 0 & 0 & 0 & \\
\hline$(3)^{m}$ & 0 & 0 & $\begin{array}{l:l}I & 0\end{array}$ & 0 & 0 & \\
\hline (4) $n$ & 0 & $E$ & 0 & 0 & 0 & \\
\hline (5) & 0 & $I: 0$ & 0 & 0 & 0 & \\
\hline$(6)^{m}$ & 0 & 0 & $0: I$ & 0 & 0 & \\
\hline (7) 1 & 1 & 0 & 0 & 0 & 0 & \\
\hline (8) 1 & -1 & 0 & 0 & 0 & 0 & \\
\hline
\end{tabular}

Figure 3.7: The matrix of derivatives of $g^{B^{*}}$ and $h^{B^{*}}$.

The structure of the proof of Theorem 3.2 is as follows. First, it is shown that for almost all games $u, M\left[g^{B^{*}, u}, h^{B^{*}, u}\right]$ is a regular constraint set, from which the manifold structure of $\mathcal{L}\left(\Gamma, p, B^{*}\right)$ follows immediately (see Chapter 2.4 for some notations and definitions from the theory of regular constraint sets and see also Herings (1997) for a first application of this theory in economics). Next it is shown that there is an open set of full measure for which the manifold structure holds. The proof of the first part is done when it is shown that for almost all games $u$, for every $\bar{x} \in M\left[g^{B^{*}, u}, h^{B^{*}, u}\right]$,

$$
\left\{\partial_{x} g_{j}^{B^{*}, u}(\bar{x})^{\top} \mid j \in J^{1}\right\} \cup\left\{\partial_{x} h_{j}^{B^{*}, u}(\bar{x})^{\top} \mid j \in J^{0}(\bar{x})\right\}
$$

is a set of independent vectors, where $J^{1}$ represents the set of rows from (1)-(4) and $J^{0}(\bar{x})$ the set of rows from (5)-(8) for which $h_{j}(\bar{x})=0$. To show this we need three lemmas, Lemmas 3.8, 3.9 and 3.10. Lemma 3.8 is used to handle points $\bar{x}$ for which $J^{0}(\bar{x})=\emptyset$, Lemma 3.9 deals with points $\bar{x}$ such that the cardinality of $J^{0}(\bar{x})$ is one, and Lemma 3.10 implies that $J^{0}(\bar{x})$ contains one element at most.

Lemma 3.8 Let a prior $p \in \Sigma$ and an admissible subset $B^{*}$ of $S^{*}$ be given. Then, for almost all $u, g^{B^{*}, u} \pitchfork\{0\}$.

Proof Consider a point $(\bar{t}, \bar{\sigma}, \bar{\lambda}, \bar{\mu}, \bar{u})$ such that $g^{B^{*}}(\bar{t}, \bar{\sigma}, \bar{\lambda}, \bar{\mu}, \bar{u})=0$. The matrix of partial derivatives of $g^{B^{*}}$ at $(\bar{t}, \bar{\sigma}, \bar{\lambda}, \bar{\mu}, \bar{u})$ is given by the rows (1)-(4) in Figure 3.7. We show first that this matrix has full row rank, from which it follows that $g^{B^{*}} \pitchfork\{0\}$.

Since $\partial_{u} v(\cdot)$ has full row rank and the derivative with respect to $u$ in (2)-(4) is zero, it is sufficient to show that the part of the matrix given by (2)-(4) has full row rank. Since the derivative 
with respect to $\lambda$ in (3) has full row rank, whereas the derivative with respect to $\lambda$ in (2) and (4) is zero, it is sufficient to show that the rows in (2) and (4) are independent. The admissibility of $B^{*}$ implies that the derivative with respect to $\sigma_{B^{*}}$ in (4) has full row rank. Since, the derivative with respect to $\sigma_{B^{*}}$ in (2) is zero, the only thing left to prove is that (2) has independent rows, which is obvious from the derivative with respect to $\sigma_{S^{*} \backslash B^{*}}$. Consequently, $g^{B^{*}} \pitchfork\{0\}$.

By the transversality theorem, Theorem 2.26 , and since $g^{B^{*}}$ is a $C^{\infty}$ function, it follows that the complement of $\left\{u \in \mathbb{R}^{n m} \mid g^{B^{*}, u} \pitchfork\{0\}\right\}$ has Lebesgue measure zero.

Lemma 3.9 Let a prior $p \in \Sigma$ and an admissible subset $B^{*}$ of $S^{*}$ be given. Moreover, let an inequality $j^{\prime} \in\left\{1, \ldots, m^{*}+2\right\}$ be given. Then, for almost all $u,\left(g^{B^{*}, u}, h_{j^{\prime}}^{B^{*}, u}\right) 历\{0\}$.

Proof Take $(\bar{t}, \bar{\sigma}, \bar{\lambda}, \bar{\mu}, \bar{u})$ such that $g^{B^{*}}(\bar{t}, \bar{\sigma}, \bar{\lambda}, \bar{\mu}, \bar{u})=0$ and $h_{j^{\prime}}^{B^{*}}(\bar{t}, \bar{\sigma}, \bar{\lambda}, \bar{\mu}, \bar{u})=0$. The matrix of partial derivatives of $\left(g^{B^{*}}, h_{j^{\prime}}^{B^{*}}\right)$ at $(\bar{t}, \bar{\sigma}, \bar{\lambda}, \bar{\mu}, \bar{u})$ is given in Figure 3.7 by the rows (1)-(4) and a single row related to $h_{j^{\prime}}^{B^{*}}$ in (5)-(8). First it is shown that this matrix has full row rank, from which it follows that $\left(g^{B^{*}}, h_{j^{\prime}}^{B^{*}}\right) \pitchfork\{0\}$.

If row $j^{\prime}$ belongs to (6), (7) or (8), then it follows from the derivative with respect to $\lambda_{S^{*} \backslash B^{*}}$ or from the derivative with respect to $t$ that row $j^{\prime}$ has rank 1 . Since all other derivatives in row $j^{\prime}$ are zero, it follows as in the proof of Lemma 3.8 that the rows of (1)-(4) together with row $j^{\prime}$ are independent. Consider the case where row $j^{\prime}$ belongs to (5). Following the first part of the proof of Lemma 3.8, it suffices to prove that (2) and (4) together with row $j^{\prime}$ are independent. Inequality $h_{j^{\prime}}^{B^{*}}$ states that $\sigma_{j^{\prime}}^{i} \geq 0, s_{j^{\prime}}^{i} \in B^{*}$, and this inequality is now required to hold with equality. Since $\sum_{j=1}^{m^{i}} \sigma_{j}^{i}-1=0$, it follows that $\left|B^{*} \cap S^{i}\right|>1$. Then the row related to $s_{j^{\prime}}^{i}$ together with (2) and (4) trivially form an independent set. Consequently, $\left(g^{B^{*}}, h_{j^{\prime}}^{B^{*}}\right) \pitchfork\{0\}$. By the transversality theorem it follows that the complement of $\left\{u \in \mathbb{R}^{n m} \mid\left(g^{B^{*}, u}, h_{j^{\prime}}^{B^{*}, u}\right) 历\{0\}\right\}$ has Lebesgue measure zero.

Lemma 3.10 Let a prior $p \in \Sigma$ and an admissible subset $B^{*}$ of $S^{*}$ be given. Moreover, let inequalities $j^{\prime}, j^{\prime \prime} \in\left\{1, \ldots, m^{*}+2\right\}$ with $j^{\prime} \neq j^{\prime \prime}$ be given. Then, for almost all $u$, $\left(g^{B^{*}, u}, h_{j^{\prime}}^{B^{*}, u}, h_{j^{\prime \prime}}^{B^{*}, u}\right) \pitchfork\{0\}$.

Proof Consider the point $(\bar{t}, \bar{\sigma}, \bar{\lambda}, \bar{\mu}, \bar{u})$ such that $g^{B^{*}}(\bar{t}, \bar{\sigma}, \bar{\lambda}, \bar{\mu}, \bar{u})=0, h_{j^{\prime}}^{B^{*}}(\bar{t}, \bar{\sigma}, \bar{\lambda}, \bar{\mu}, \bar{u})=0$, and $h_{j^{\prime \prime}}^{B^{*}}(\bar{t}, \bar{\sigma}, \bar{\lambda}, \bar{\mu}, \bar{u})=0$. The matrix of partial derivatives of $\left(g^{B^{*}}, h_{j^{\prime}}^{B^{*}}, h_{j^{\prime \prime}}^{B^{*}}\right)$ at $(\bar{t}, \bar{\sigma}, \bar{\lambda}, \bar{\mu}, \bar{u})$ is given in Figure 3.7 by the rows (1)-(4) and two rows related to $h_{j^{\prime}}^{B^{*}}$ and $h_{j^{\prime \prime}}^{B^{*}}$ in (5)-(8). First it is shown that this matrix has full row rank, from which it follows that $\left(g^{B^{*}}, h_{j^{\prime}}^{B^{*}}, h_{j^{\prime \prime}}^{B^{*}}\right) 币\{0\}$.

The case where the two rows are not equal to (7) and (8) is similar to the proof of Lemma 3.9. Rows (7) and (8) are not independent. However, they cannot be binding simultaneously, because then it holds that $t=0$ and $t=1$. Consequently, $\left(g^{B^{*}}, h_{j^{\prime}}^{B^{*}}, h_{j^{\prime \prime}}^{B^{*}}\right) 币\{0\}$. It follows that the complement of the set $\left\{u \in \mathbb{R}^{n m} \mid\left(g^{B^{*}, u}, h_{j^{\prime}}^{B^{*}, u}, h_{j^{\prime \prime}}^{B^{*}, u}\right) \pitchfork\{0\}\right\}$ has Lebesgue measure zero.

Proof of Theorem 3.2 Fix $n$ and $m^{i}, i \in N$. Fix a prior $p \in \Sigma$ and an admissible subset 
$B^{*}$ of $S^{*}$. The set $U$ consisting of $u \in \mathbb{R}^{m n}$ such that $g^{B^{*}, u} \pitchfork\{0\},\left(g^{B^{*}, u}, h_{j^{\prime}}^{B^{*}, u}\right) \pitchfork\{0\}$ for all $j^{\prime} \in\left\{1, \ldots, m^{*}+2\right\}$, and $\left(g^{B^{*}, u}, h_{j^{\prime}}^{B^{*}, u}, h_{j^{\prime \prime}}^{B^{*}, u}\right) \pitchfork\{0\}$ for all $j^{\prime}, j^{\prime \prime} \in\left\{1, \ldots, m^{*}+2\right\}$ with $j^{\prime} \neq j^{\prime \prime}$, has full measure by Lemmas 3.8, 3.9 and 3.10.

Consider any $u \in U$. It has to be shown that $M\left[g^{B^{*}, u}, h^{B^{*}, u}\right]$ is a regular constraint set. Consider any $\bar{x} \in M\left[g^{B^{*}, u}, h^{B^{*}, u}\right]$. Then, it has to be shown that

$$
\left\{\partial_{x} g_{j}^{B^{*}, u}(\bar{x})^{\top} \mid j \in J^{1}\right\} \cup\left\{\partial_{x} h_{j}^{B^{*}, u}(\bar{x})^{\top} \mid j \in J^{0}(\bar{x})\right\}
$$

is a set of independent vectors. When $J^{0}(\bar{x})=\emptyset$, this is a consequence of Lemma 3.8. When $\left|J^{0}(\bar{x})\right|=1$, this is a consequence of Lemma 3.9. Suppose $\left|J^{0}(\bar{x})\right| \geq 2$ and choose two elements, say $j^{\prime}$ and $j^{\prime \prime}$ from $J^{0}(\bar{x})$. It follows from Lemma 3.10 that

$$
\left\{\partial_{x} g_{j}^{B^{*}, u}(\bar{x})^{\top} \mid j \in J^{1}\right\} \cup\left\{\partial_{x} h_{j^{\prime}}^{B^{*}, u}(\bar{x})^{\top}, \partial_{x} h_{j^{\prime \prime}}^{B^{*}, u}(\bar{x})^{\top}\right\}
$$

is a set of independent vectors, which leads to a contradiction as the set contains $2 m^{*}+n+2$ vectors of dimension $2 m^{*}+n+1$. Consequently, $\left|J^{0}(\bar{x})\right| \leq 1$, and $M\left[g^{B^{*}, u}, h^{B^{*}, u}\right]$ is a regular constraint set. It follows that $\mathcal{O}\left(\Gamma, p, B^{*}\right)$ is a 1-dimensional manifold with boundary, and that the boundary of $\mathcal{O}\left(\Gamma, p, B^{*}\right)$ is given by the elements $\bar{x} \in M\left[g^{B^{*}, u}, h^{B^{*}, u}\right]$ for which $\left|J^{0}(\bar{x})\right|=1$. Therefore, a point in $\mathcal{O}\left(\Gamma, p, B^{*}\right)$ is a boundary point if and only if exactly one of the inequalities in (5)-(8) holds with equality. These properties carry over to $\mathcal{L}\left(\Gamma, p, B^{*}\right)$ since it is diffeomorphic to $\mathcal{O}\left(\Gamma, p, B^{*}\right)$ by Theorem 3.1 .

Compactness of $\mathcal{L}\left(\Gamma, p, B^{*}\right)$ is obvious.

It remains to be shown that there is an open set of games and priors with full Lebesgue measure for which the asserted properties hold. Let $\Omega$ be the set of all noncooperative games and priors and let $\Omega^{B^{*}}$ be the subset of $\Omega$ consisting of all pairs $(\Gamma, p)$ for which the transversality statements in Lemma 3.8, Lemma 3.9 and Lemma 3.10 hold. It has already been shown that $\Omega \backslash \Omega^{B^{*}}$ has Lebesgue measure zero. If $(\Gamma, p) \in \Omega \backslash \Omega^{B^{*}}$, then there exist $(t, \sigma, \lambda, \mu) \in \mathbb{R}^{2 m^{*}+n+1}$ such that $(t, \sigma, \lambda, \mu, \Gamma, p)$ belongs to the set $\Xi^{B^{*}}$ defined by

$$
\begin{aligned}
& \Xi^{B^{*}}=\left\{(y, \Gamma, p) \in \mathbb{R}^{2 m^{*}+n+1} \times \Omega \mid\right. \\
& g^{B^{*}, u, p}(y)=0 \quad \text { and } \operatorname{rank}\left(\partial g^{B^{*}, u, p}(y)\right) \leq 2 m^{*}+n-1, \\
& \exists j^{\prime}, g^{B^{*}, u, p}(y)=h_{j^{\prime}}^{B^{*}, u, p}(y)=0 \quad \text { and } \quad \operatorname{rank}\left(\begin{array}{c}
\partial g^{B^{*}, u, p}(y) \\
\partial h_{j^{\prime}}^{B^{*}, u, p}(y)
\end{array}\right) \leq 2 m^{*}+n, \\
& \left.\exists j^{\prime}, j^{\prime \prime}, j^{\prime} \neq j^{\prime \prime}, g^{B^{*}, u, p}(y)=h_{j^{\prime}}^{B^{*}, u, p}(y)=h_{j^{\prime \prime}}^{B^{*}, u, p}(y)=0 \quad\right\} .
\end{aligned}
$$

The notation $g^{B^{*}, u, p}$ and $h^{B^{*}, u, p}$ makes the dependence on the prior $p$ explicit. The set $\Xi^{B^{*}}$ is closed since it is obtained by finite unions and intersections of closed sets. Define the projection $\pi: \Xi^{B^{*}} \rightarrow \Omega$ by

$$
\pi(t, \sigma, \lambda, \mu, \Gamma, p)=(\Gamma, p) \quad \text { for all }(t, \sigma, \lambda, \mu, \Gamma, p) \in \Xi^{B^{*}} .
$$


It holds that $\pi\left(\Xi^{B^{*}}\right)=\Omega \backslash \Omega^{B^{*}}$. Next it is shown that $\pi$ is proper, i.e. that $\pi^{-1}(K)$ is compact if $K$ is a compact subset of $\Omega$. Let $K$ be a compact subset of $\Omega$. Clearly, $\pi^{-1}(K)$ is a closed subset of the set $\Xi^{B^{*}}$, which is easily seen to be compact, and therefore $\pi^{-1}(K)$ is compact. Since the image by a continuous proper mapping of a closed set is closed, it follows that $\pi\left(\Xi^{B^{*}}\right)=\Omega \backslash \Omega^{B^{*}}$ is closed, so $\Omega^{B^{*}}$ is open. Since there is a finite number of admissible subsets $B^{*}$ of $S^{*}$, the intersection of the sets $\Omega^{B^{*}}$ over all admissible subsets $B^{*}$ is an open set of full Lebesgue measure of games and priors with the properties as stated in the theorem.

Proof of Theorem 3.4 Fix any game and any prior in the open set of games $\Gamma \in \mathcal{G}$ and priors $p \in \Sigma$ with full Lebesgue measure of Theorem 3.2. For any $B^{*}$ it holds that $\mathcal{L}\left(\Gamma, p, B^{*}\right)$ is a compact 1-dimensional $C^{\infty}$ manifold with boundary, so it consists of a finite number of arcs and loops. Each arc in $\mathcal{L}\left(\Gamma, p, B^{*}\right)$ has two endpoints. If it has an endpoint as in alternative (i) of Theorem 3.3, then it does not belong to any $\mathcal{L}\left(\Gamma, p, \bar{B}^{*}\right)$ for $\bar{B}^{*} \neq B^{*}$ and lies in $\{0,1\} \times \Sigma$. Therefore, it is a boundary point of $\mathcal{L}(\Gamma, p)$. If an arc has an endpoint as in alternative (ii) of Theorem 3.3, then it belongs to $(0,1) \times \Sigma$ and there exists exactly one $\bar{B}^{*} \neq B^{*}$ such that this endpoint is also an endpoint of an $\operatorname{arc}$ in $\mathcal{L}\left(\Gamma, p, \bar{B}^{*}\right)$. This arc in $\mathcal{L}\left(\Gamma, p, \bar{B}^{*}\right)$ has another endpoint that either satisfies alternative (i) of Theorem 3.3 or alternative (ii) of Theorem 3.3. In the former case an endpoint of $\mathcal{L}(\Gamma, p)$ in $\{0,1\} \times \Sigma$ has been found. In the latter case, the endpoint belongs to $(0,1) \times \Sigma$ and there is exists exactly one $\tilde{B}^{*} \neq \bar{B}^{*}$ such that this endpoint is also an endpoint of an arc in $\mathcal{L}\left(\Gamma, p, \tilde{B}^{*}\right)$, and so on.

Since there is a finite number of admissible subsets $B^{*} \subseteq S^{*}$, and each $\mathcal{L}\left(\Gamma, p, B^{*}\right)$ consists of finitely many arcs and loops, it will either be the case that eventually an arc is generated with an endpoint as in alternative (i) of Theorem 3.3, or an arc is generated that has been generated before. In the latter case, a piecewise $C^{\infty}$ loop of $\mathcal{L}(\Gamma, p)$ is found. In the former case, the finite chain of arcs constitutes a piecewise $C^{\infty}$ arc of $\mathcal{L}(\Gamma, p)$ with endpoints belonging to $\{0,1\} \times \Sigma$. Indeed, starting from the endpoint already found in $\{0,1\} \times \Sigma$ and generating the chain of arcs in $\mathcal{L}\left(\Gamma, p, B^{*}\right)$ for various admissible subsets $B^{*}$, will either lead to another endpoint of $\mathcal{L}(\Gamma, p)$ in $\{0,1\} \times \Sigma$ or to the generation of an endpoint of some $\mathcal{L}\left(\Gamma, p, B^{*}\right)$ in $(0,1) \times \Sigma$ that has been generated before. The latter case is excluded as this point would be an endpoint of three different arcs, which violates Theorem 3.3. It follows that $\mathcal{L}(\Gamma, p)$ is a compact 1-dimensional piecewise $C^{\infty}$ manifold with boundary, where the boundary is given by the intersection of $\mathcal{L}(\Gamma, p)$ and $\{0,1\} \times \Sigma$. The argument to show this is nothing but a nonlinear version of the door-in door-out principle of Lemke and Howson (1964).

It remains to be shown that there is a unique boundary point in $\{0\} \times \Sigma$. Notice that $(0, \sigma) \in$ $\mathcal{L}(\Gamma, p)$ if and only if for all $i, \sigma^{i}$ is a best response against the prior $p$. If there would be more than one best response for some player, then there would be at least two pure best responses for some player when $t=0$, which contradicts Lemma 3.10. 
Lemma 3.11 There exists an open set of games and priors $(\Gamma, p) \in \mathcal{G} \times \Sigma$ with full Lebesgue measure such that $\partial_{(t, \alpha, \mu)} \mathcal{H}(\bar{t}, \bar{\alpha}, \bar{\mu})$ has full rank for all points $(\bar{t}, \bar{\alpha}, \bar{\mu})$ with $\mathcal{H}(\bar{t}, \bar{\alpha}, \bar{\mu})=0$, $\partial_{(\alpha, \mu)} \mathcal{H}(0, \bar{\alpha}, \bar{\mu})$ has full rank for all $(\bar{\alpha}, \bar{\mu})$ with $\mathcal{H}(0, \bar{\alpha}, \bar{\mu})=0$, and $\partial_{(\alpha, \mu)} \mathcal{H}(1, \bar{\alpha}, \bar{\mu})$ has full rank for all $(\bar{\alpha}, \bar{\mu})$ with $\mathcal{H}(1, \bar{\alpha}, \bar{\mu})=0$.

Proof Fix any game and any prior in the open set of games $\Gamma \in \mathcal{G}$ and priors $p \in \Sigma$ with full Lebesgue measure of Theorem 3.2. In Figure 3.8 the Jacobian of $\mathcal{H}$ is shown, where

$$
E\left(i, s_{j^{\prime}}^{i^{\prime}}\right)= \begin{cases}1 & \text { if } i=i^{\prime} \\ 0 & \text { otherwise }\end{cases}
$$

and

$$
E_{\alpha}\left(i, s_{j^{\prime}}^{i^{\prime}}\right)=\left\{\begin{array}{ll}
2 \alpha_{j^{\prime}}^{i^{\prime}} & \text { if } i=i^{\prime} \\
0 & \text { otherwise }
\end{array} .\right.
$$

To simplify the exposition of this Jacobian, the strategies $s_{j}^{i}$ are first ordered according to whether $\alpha_{j}^{i}$ is positive or not.

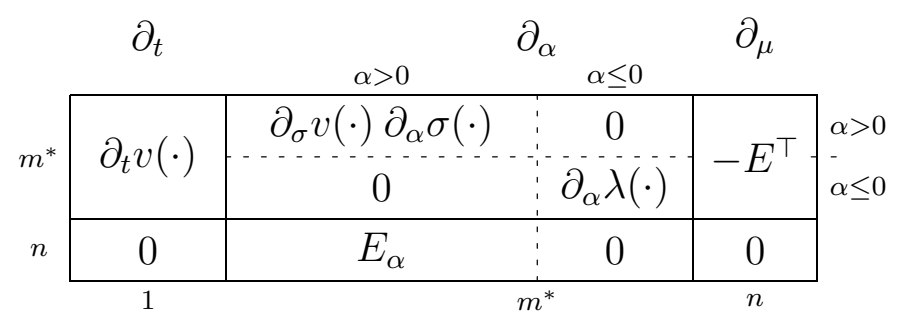

Figure 3.8: The Jacobian of the homotopy function $\mathcal{H}$.

The Jacobian of $\mathcal{H}$ can be decomposed as the product of the two matrices shown in Figure 3.9. The first matrix equals the rows in (1) and (4) of Figure 3.7 when $B^{*}=\left\{s_{j}^{i} \in S^{*} \mid \alpha_{j}^{i}>0\right\}$. Because $g^{B^{*}, u} \pitchfork\{0\}$, this matrix has full rank. In the second matrix, the notation $\Lambda(2 \alpha)$ is used to represent a diagonal matrix with the components of the vector $2 \alpha$ on the diagonal. The second matrix contains at least $m^{*}$ zero rows. Since it cannot be the case that two variables $\alpha_{j}^{i}$ are zero at the same time, the second matrix contains at most $m^{*}+1$ zero rows.

Delete the $m^{*}$ zero rows in the middle part of the second matrix and the corresponding columns in the first matrix. The product of the resulting matrices remains equal to the Jacobian of $\mathcal{H}$. By deleting the zero rows in the second and the corresponding columns in the first matrix both matrices do not drop rank. For the second matrix this is obvious. For the first matrix, note that after deletion of the columns, it is equal to the submatrix of Figure 3.7 that results after applying Gaussian elimination to the rows in (1) and (4) by the rows in (2) and (3), and therefore has full rank. 


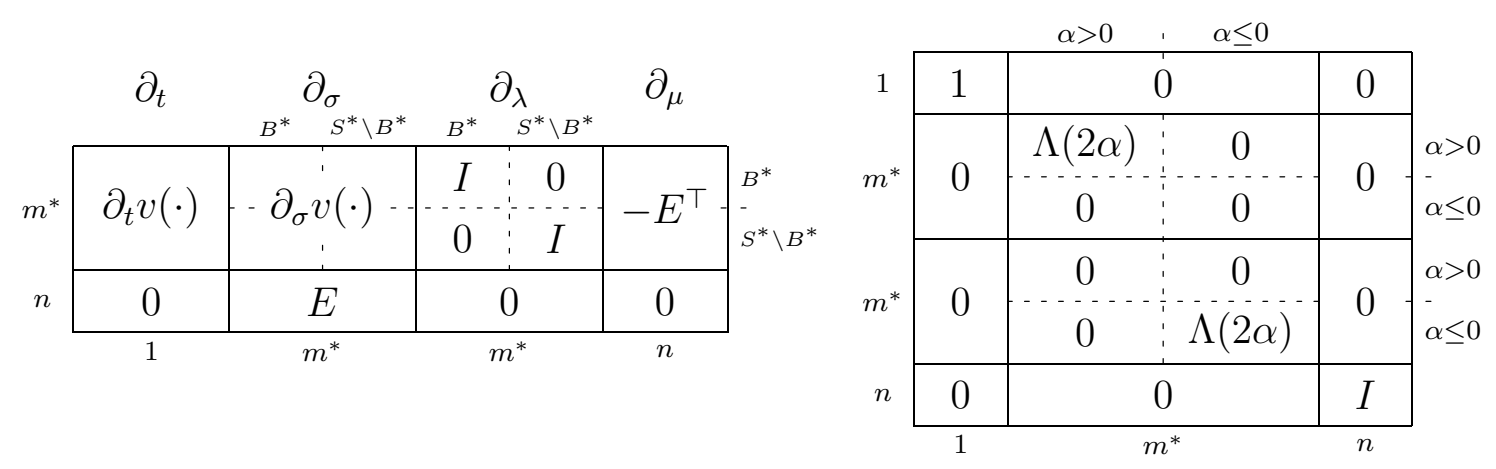

Figure 3.9: Decomposition of the Jacobian of $\mathcal{H}$ into two matrices.

If $\alpha_{j}^{i} \neq 0$ for all strategies $s_{j}^{i}$, then the remaining second matrix is a diagonal matrix with nonzero entries on the diagonal, so it has full rank. As a consequence, the product of the remaining matrices and thereby the Jacobian of $\mathcal{H}$ has full rank.

If $\alpha_{\ell}^{k}=0$ for a certain strategy $s_{\ell}^{k}$, then the remaining second matrix has still one zero row. After deletion of this row, the remaining second matrix still has full rank. After deletion of the corresponding column, the remaining first matrix also has full rank, as it is equal to the submatrix of Figure 3.7 that results after applying Gaussian elimination to the rows in (1) and (4) by the rows in (2) and (3), and the row in (6) corresponding to $s_{\ell}^{k}$. As the remaining first matrix is a square matrix, it follows that the product of the remaining matrices, and thereby the Jacobian of the homotopy function, has full rank. Consequently, $\partial_{(t, \alpha, \mu)} \mathcal{H}(\bar{t}, \bar{\alpha}, \bar{\mu})$ has full rank for all $(\bar{t}, \bar{\alpha}, \bar{\mu})$ with $\mathcal{H}(\bar{t}, \bar{\alpha}, \bar{\mu})=0$.

For proving that $\partial_{(\alpha, \mu)} \mathcal{H}(0, \bar{\alpha}, \bar{\mu})$ has full rank for all $(\bar{\alpha}, \bar{\mu})$ with $\mathcal{H}(0, \bar{\alpha}, \bar{\mu})=0$, and that $\partial_{(\alpha, \mu)} \mathcal{H}(1, \bar{\alpha}, \bar{\mu})$ has full rank for all $(\bar{\alpha}, \bar{\mu})$ with $\mathcal{H}(1, \bar{\alpha}, \bar{\mu})=0$, consider the matrix in Figure 3.8 without the first column. That matrix can be decomposed as the product of the matrices of Figure 3.9 with the first column of the first matrix and both the first row and the first column of the second matrix deleted. The resulting second matrix contains exactly $m^{*}$ zero rows, as none of the variables $\alpha_{j}^{i}$ equals zero when $t=0$ or $t=1$. Delete the $m^{*}$ zero rows of the second matrix and the corresponding columns in the first matrix. The product of the resulting matrices remains equal to the Jacobian of $\mathcal{H}$. The resulting first matrix is equal to the submatrix of Figure 3.7 that is obtained after applying Gaussian elimination to the rows in (1) and (4) by the rows in (2) and (3), and the row in (7) when $t=0$ or the row in (8) when $t=1$. Therefore, both the resulting first matrix and the resulting second matrix are square matrices with full rank, and so is their product. Consequently, $\partial_{(\alpha, \mu)} \mathcal{H}(0, \bar{\alpha}, \bar{\mu})$ has full rank for all $(\bar{\alpha}, \bar{\mu})$ with $\mathcal{H}(0, \bar{\alpha}, \bar{\mu})=0$, and $\partial_{(\alpha, \mu)} \mathcal{H}(1, \bar{\alpha}, \bar{\mu})$ has full rank for all $(\bar{\alpha}, \bar{\mu})$ with $\mathcal{H}(1, \bar{\alpha}, \bar{\mu})=0$.

Proof of Theorem 3.7 Fix any game and any prior in the open set of games $\Gamma \in \mathcal{G}$ and priors $p \in \Sigma$ with full Lebesgue measure of Theorem 3.2. Consider the following system of equalities 
and inequalities,

$$
\begin{aligned}
& \mathcal{H}(t, \alpha, \mu)=0, \\
& t \geq 0, \\
& -t+1 \geq 0 .
\end{aligned}
$$

The set of solutions to this system equals $\mathcal{H}^{-1}(\{0\})$. It is a regular constraint set by Lemma 3.11 and is therefore a 1-dimensional $C^{1}$ manifold with boundary as described in the theorem. The compactness of $\mathcal{H}^{-1}(\{0\})$ follows trivially. The uniqueness of the boundary point in $\{0\} \times \mathbb{R}^{m^{*}} \times$ $\mathbb{R}^{n}$ carries over from Theorem 3.4. 


\section{Chapter 4}

\section{Computation of All Equilibria}

In this chapter an algorithm is presented to compute all Nash equilibria for games in normal form on the only premises that the number of Nash equilibria is finite. The algorithm relies on decomposing the game by means of support-sets. For each support-set, the set of totally mixed equilibria of the subgame that results by restricting the players to strategies in the support-set, can be characterized by a system of polynomial equations and inequalities. By solving those systems for each support-set, all equilibria are found. The algorithm belongs to the class of homotopy-methods and is implementable. Finally, several techniques to speed up computations are proposed.

\subsection{Introduction}

In the previous chapter it was argued that a further application of game theory to research fields in which conflicts between agents arise may stagnate when the tools are found not to be powerful enough. One such tool is an algorithm to compute equilibria and select among equilibria. For many purposes, having an algorithm to compute a single sample equilibrium might be unsatisfactory. Even if the algorithm is able to select within the complete set of equilibria on perfectness or some other refinement criterion, it cannot be neglected that there might exist another equilibrium that is more salient. For some refinements, for example risk dominance, a candidate equilibrium has to be compared with the other equilibria of the game. Even when an equilibrium for a game is found, there might exist multiple equilibria with different implications with regard to the original problem under consideration. All are motivations for having an algorithm to compute all equilibria.

For bimatrix games, efficient and implementable algorithms to compute all equilibria exist. For bimatrix games in which one player has exactly two strategies at its disposal, an algorithm to compute the complete set of Nash equilibria is developed by Borm, Gijsberts, and Tijs (1987). For the general class of bimatrix games, algorithms are developed by Dickhaut and 
Kaplan (1993) and Kostreva and Kinard (1991).

The algorithm which is implemented in Gambit ${ }^{1}$ allows for finding all Nash equilibria of an $n$-person normal form game via the Liapunov function method described in McKelvey (1996). This is a continuously differentiable nonnegative function whose zeros coincide with the set of Nash equilibria of the game. A standard descent algorithm is used to find a constrained local minimum of the function from any given starting location. All global minima, the local minima with function value zero, are Nash equilibria of the game under inspection. But, these functions for computing 'all' equilibria will only find all equilibria (for generic games) in a weak probabilistic sense: Given any number less than one, there is an amount of time such that if the algorithm is run for at least that amount of time it will find all solutions with probability higher than that given number. For a general survey on the computation of equilibria, see McKelvey and McLennan (1996).

The approach enhanced in the present chapter is again the homotopy approach. The set of Nash equilibria can be represented as the set of solutions to a system of polynomial equations and inequalities. When the system is decomposed by means of all possible carrier structures only equations remain for which the solutions have to be computed and subsequently have to be checked whether they really are equilibria. For the computation of the solutions to the systems of polynomial equations, the homotopy approach is chosen. For homotopy continuation algorithms to solve systems of equations and inequalities of multivariate polynomials a large library of literature is present.

As is well-known, see for instance McLennan (1999), the number of Nash equilibria increases exponentially in the size of the game. Therefore, the algorithm that is proposed is by definition exponentially. Exponential algorithms are sometimes loosely described as impractical. If all problems of interest were large in scale, then indeed such algorithms are not very worthwhile.

The algorithm proposed in this chapter has the property that it generates more and more Nash equilibria during its execution, so there is no need to wait until it finally terminates. There is also the flexibility to start searching for particular equilibria, like Nash equilibria in pure strategies, or Nash equilibria in completely mixed strategies, before turning to others. It is also possible to efficiently apply parallel computers to speed up computations.

An alternative approach that is addressed in this chapter is one for which no decomposition is needed. It directly computes the solutions to a set of polynomial equations which again have to be checked for satisfying some inequalities.

This chapter is organized as follows. Some notations, definitions and general results are given in Section 4.2. In Section 4.3 a method to compute all equilibria is proposed. Section 4.4 deals in detail with the implementation of the proposed algorithm. In Section 4.5 the algorithm we

\footnotetext{
${ }^{1}$ Gambit is a library of game theory software and tools for the construction and analysis of finite extensive and normal form games. See http://www.hss.caltech.edu/ gambit/Gambit.html.
} 
propose is described explicitly. In Section 4.6 an alternative method is mentioned. Section 4.7 concludes.

\subsection{Number of equilibria}

Consider an $n$-person noncooperative game in normal form $\Gamma=\left\langle N,\left\{S^{i}\right\}_{i \in N},\left\{u^{i}\right\}_{i \in N}\right\rangle$ as defined in Chapter 3. All notations used are similar to that of the previous chapter except when mentioned otherwise.

From the existence of a Nash equilibrium, it is clear that for any game the number of equilibria is larger than or equal to one. It can also be shown that the number of Nash equilibria is generically odd, see for instance Harsanyi (1973). The exact number of equilibria is not known and may vary among games. However, some results are known on the number of equilibria of a normal form game with given size. For generic games, McKelvey and McLennan (1997) have determined the maximal number of totally mixed Nash equilibria and in McLennan (1997) the maximal number of pure Nash equilibria is determined. McLennan (1999) presents a formula for the expected number of Nash equilibria for a random normal form game for given (finite and nonempty) sets of players and pure strategies. In Table 4.1, for some numbers of players ( $n$ ) and some common number of strategies $(m)$, an estimation of the mean number of Nash equilibria is displayed with between brackets the standard error of the estimation. ${ }^{2}$

\begin{tabular}{cccccc}
\hline$n \backslash m$ & 2 & 3 & 4 & 5 & 6 \\
\hline 2 & $1.31(0.13)$ & $1.52(0.12)$ & $1.77(0.18)$ & $2.64(0.44)$ & $2.61(0.52)$ \\
3 & $2.15(0.20)$ & $3.76(0.33)$ & $12.66(1.13)$ & $27.23(2.78)$ & $65.69(4.19)$ \\
4 & $4.49(0.40)$ & $18.01(1.22)$ & $82.49(3.87)$ & $440.02(18.86)$ & \\
5 & $6.98(0.43)$ & $81.82(3.32)$ & $879.24(32.94)$ & & \\
6 & $15.75(0.96)$ & $401.61(10.77)$ & & & \\
\hline
\end{tabular}

Table 4.1: Mean number of equilibria.

McLennan (1999) concludes from these numbers that the average number of Nash equilibria grows more rapidly than the size of the game, if the size of the game is measured by the number of pure strategy tuples times the number of players (which equals the number of payoffs in the game) and that the table clearly conveys the impression that the mean number of equilibria is greater when a large number of players each have a small number of pure strategies than the other way around.

\footnotetext{
${ }^{2}$ Although Table 4.1 looks quite similar to Table 3.3 of the previous chapter, the presentation is transposed and other pairs $(n, m)$ are chosen.
} 


\subsection{Equilibria as solutions to systems of equations}

In this section a method is presented to reformulate the problem of finding all Nash equilibria of a normal form game to finding all (finite nonnegative real) zeros of systems of multivariate polynomials. First a normal form game is decomposed by means of all possible carriers and secondly all totally mixed equilibria of the games that result by restricting the players to choose strategies within the predescribed carriers are computed. The 'subgames' resulting from carriers where only two players have more than one strategy are equivalent to bimatrix games. For these subgames existing algorithms developed for bimatrix games, such as for instance the algorithms proposed in Dickhaut and Kaplan (1993) and in Kostreva and Kinard (1991), can be applied and might improve (decrease) computation time.

Let a subset $D^{*}$ of $S^{*}$ be given with the property that for every player $i$ there is at least one pure strategy $s_{j}^{i}$ in $D^{*}$, i.e. $D^{i}=D^{*} \cap S^{i} \neq \emptyset$ for every player $i .^{3}$ Such a set $D^{*}$ is called admissible. Admissible subsets $D^{*}$ are used to decompose $\mathrm{NE}(\Gamma)$ in subsets $\operatorname{NE}\left(\Gamma, D^{*}\right)$, where $\operatorname{NE}\left(\Gamma, D^{*}\right)$ contains those elements of $\mathrm{NE}(\Gamma)$ where only strategies in $D^{*}$ are played with positive probability and all strategies in $D^{*}$ are best responses, i.e.

$$
\begin{aligned}
\mathrm{NE}\left(\Gamma, D^{*}\right)=\{\sigma \in \mathrm{NE}(\Gamma) \mid & s_{j}^{i} \notin D^{*} \Rightarrow \sigma_{j}^{i}=0 \\
s_{j}^{i} & \left.\in D^{*} \Rightarrow s_{j}^{i} \in \operatorname{argmax}_{s_{\ell}^{i} \in S^{i}} u^{i}\left(\sigma^{-i}, s_{\ell}^{i}\right)\right\} .
\end{aligned}
$$

The situation where a Nash equilibrium $\sigma$ is an element of two different sets $\operatorname{NE}\left(\Gamma, D^{*}\right)$ is a knife-edge case. It can only occur if there is for some player $i$ an optimal strategy which is played with zero probability. It is easily seen that

$$
\mathrm{NE}(\Gamma)=\bigcup_{D^{*}} \mathrm{NE}\left(\Gamma, D^{*}\right)
$$

An admissible subset $D^{*}$ of $S^{*}$ determines a subgame $\Gamma_{\mid D^{*}}=\left\langle N,\left\{D^{i}\right\}_{i \in N},\left\{u^{i}\right\}_{i \in N}\right\rangle$ with $D^{i}=$ $\left\{d_{1}^{i}, \ldots, d_{\left|D^{i}\right|}^{i}\right\}$ the set of pure strategies of player $i$ and the payoff functions restricted to the set $D=\chi_{i \in N} D^{i}$. A mixed strategy of player $i$ is a probability distribution on $D^{i}$. The set of mixed strategies for player $i$ will be denoted by $\Delta^{i}$, with generic element $\delta^{i}$, and we define $\Delta=\chi_{i \in N} \Delta^{i}$

Given an admissible subset $D^{*}$, define

$$
\mathcal{E}\left(\Gamma_{\mid D^{*}}\right)=\left\{\delta \in \Delta \mid D^{i}=\operatorname{argmax}_{d_{\ell}^{i} \in D^{i}} u^{i}\left(\delta^{-i}, d_{\ell}^{i}\right) \quad \text { for all } i \in N\right\}
$$

as the set of all Nash equilibria $\delta$ of subgame $\Gamma_{\mid D^{*}}$ with the property that for all players $i \in N$ it holds that all strategies from $D^{i}$ are best responses to $\delta^{-i}$. Note that for all $\delta \in \operatorname{NE}\left(\Gamma, D^{*}\right)$ it

\footnotetext{
${ }^{3}$ Note that this chapter uses the ' $D$ ' to denote admissible subsets rather than the ' $B$ ' which was used in the previous chapter.
} 
holds that $\delta \in \mathcal{E}\left(\Gamma_{\mid D^{*}}\right)$, i.e. $\mathrm{NE}\left(\Gamma, D^{*}\right) \subseteq \mathcal{E}\left(\Gamma_{\mid D^{*}}\right){ }^{4}$ Elements of $\mathcal{E}\left(\Gamma_{\mid D^{*}}\right)$ are not necessarily elements of $\operatorname{NE}\left(\Gamma, D^{*}\right)$, since there may exist an $s^{i} \in S^{*} \backslash D^{*}$ with $u^{i}\left(\delta^{-i}, s^{i}\right)>u^{i}(\delta)$. Therefore it holds that $\mathrm{NE}\left(\Gamma, D^{*}\right)=\mathcal{E}\left(\Gamma_{\mid D^{*}}\right) \cap \mathrm{NE}(\Gamma)$.

If $\delta \in \mathcal{E}\left(\Gamma_{\mid D^{*}}\right)$, then

$$
\begin{aligned}
& u^{i}\left(\delta^{-i}, d_{j}^{i}\right)-u^{i}\left(\delta^{-i}, d_{\ell}^{i}\right)=0, \quad\left(d_{j}^{i}, d_{\ell}^{i} \in D^{i}, i \in N\right), \\
& \sum_{d_{j}^{i} \in D^{i}} \delta_{j}^{i}-1=0, \quad(i \in N) .
\end{aligned}
$$

Fix one element $\tilde{d}^{i} \in D^{i}$ for each player $i$. This is possible because of the admissibility of the set $D^{*}$. Then the set of solutions to the set of equations above is equivalent to the set of solutions of the following system of multilinear equations:

$$
\begin{aligned}
& u^{i}\left(\delta^{-i}, \tilde{d}^{i}\right)-u^{i}\left(\delta^{-i}, d^{i}\right)=0, \quad\left(d^{i} \in D^{i} \backslash\left\{\tilde{d}^{i}\right\}, i \in N\right), \\
& \sum_{d_{j}^{i} \in D^{i}} \delta_{j}^{i}-1=0, \quad(i \in N) .
\end{aligned}
$$

In total, this system has $\sum_{i \in N}\left(\left|D^{i}\right|-1\right)+n=\left|D^{*}\right|$ equations and $\left|D^{*}\right|$ unknows. What one expects is a zero-dimensional solution set. To state this differently, one expects that the set of solutions to the system (1)-(2) consists only of a finite number of isolated points.

As already mentioned in the previous chapter, a normal form game can be parameterized by its payoffs; any game $\Gamma$ is determined by the set of players, number of actions per player, and some vector $u$ containing the payoffs of the game. When it is said that a property holds for (almost) every game $\Gamma$, it means that for any specification of the set of players, and the number of pure strategies per player, the property holds for (almost) every vector $u$ that parameterizes this game $\Gamma$.

For every vector $u \in \mathbb{R}^{n|S|}$ and admissible subset $D^{*}$, define the function $F^{D^{*}, u}: \mathbb{R}^{\left|D^{*}\right|} \rightarrow$ $\mathbb{R}^{\left|D^{*}\right|}$ by the left-hand side of (1)-(2), i.e.

$$
F^{D^{*}, u}(\delta)=\left(\begin{array}{cc}
u^{i}\left(\delta^{-i}, \tilde{d}^{i}\right)-u^{i}\left(\delta^{-i}, d^{i}\right) & \left(d^{i} \in D^{i} \backslash\left\{\tilde{d}^{i}\right\}, i \in N\right) \\
\sum_{d_{j}^{i} \in D^{i}} \delta_{j}^{i}-1 & (i \in N)
\end{array}\right) .
$$

The set of solutions $\mathcal{E}\left(\Gamma_{\mid D^{*}}\right)$ to the system (1)-(2) is a subset of the set of solutions to $F^{D^{*}, u}(\delta)=$ 0 . In fact, if $\delta \in \mathcal{E}\left(\Gamma_{\mid D^{*}}\right)$, then $\delta$ is a nonnegative real solution of $F^{D^{*}, u}(\delta)=0$. An element $\delta \in \mathbb{C}^{\left|D^{*}\right|}$ is an element of $\mathcal{E}\left(\Gamma_{\mid D^{*}}\right)$ if it solves (1)-(2), and

$$
\text { (a) } \quad \delta_{j}^{i} \in \mathbb{R}_{+}, \quad\left(d_{j}^{i} \in D^{i}, i \in N\right) .
$$

It follows that $\delta \in \mathbb{C}^{\left|D^{*}\right|}$ is an element of $\mathrm{NE}\left(\Gamma, D^{*}\right)$, and therefore a Nash equilibrium of the game $\Gamma$, if and only if it solves (1)-(2), (a), and

$$
u^{i}(\delta)-u^{i}\left(\delta^{-i}, s^{i}\right) \geq 0, \quad\left(s^{i} \in S^{i} \backslash D^{i}, i \in N\right) .
$$

\footnotetext{
${ }^{4}$ In fact, $\delta$ is not an element of $\Sigma$, but from $\Delta$. When we use a $\delta$ from $\Sigma$ we actually mean $\sigma(\delta)$ from $\Sigma$ where $\sigma(\delta)$ is the trivial extension of $\delta$ in $\Delta: \sigma_{j}^{i}(\delta)=\delta_{j}^{i}$ if $s_{j}^{i} \in D^{*}$ and $\sigma_{j}^{i}(\delta)=0$ otherwise.
} 
Since (1) consists of $\left|D^{*}\right|-n$ polynomials of degree $n-1$ and (2) consists of $n$ polynomials of degree 1 , the total degree of the system $(1)-(2)$ is $(n-1)^{\left|D^{*}\right|-n}$ as being the product of the degrees of the individual equations. The theorem of Bezout says that the number of solutions and solutions at infinity, counting multiplicities, is equal to the total degree of the system (see Subsection 4.4.1).

Theorem 4.1 When the number of solutions in $\mathbb{C}^{\left|D^{*}\right|}$ to $F^{D^{*}, u}=0$ is finite, it equals $(n-1)^{\left|D^{*}\right|-n}$ if solutions are counted by multiplicity and infinite solutions are counted.

The reason that the analysis is done in the complex space is because the number of complex solutions to $F^{D^{*}, u}(\delta)=0$ is known when the solution set consists only of a finite number of isolated points, and so it is easily checked if all solutions have been found. While in practice the interest goes out to finding all solutions to the system $(1)-(2)$ that satisfy conditions $(a)-(b)$, by finding all solutions with their multiplicities, one can be sure that all solutions to $F^{D^{*}, u}(\delta)=0$, or to (1)-(2), with the desired properties (Nash equilibria) have been found.

The number of zeros we calculate in total is the sum of the zeros to $F^{D^{*}, u}$ over all admissible subsets $D^{*}$ and is equal to

$$
\sum_{k=n}^{\left|S^{*}\right|} A(k)(n-1)^{(k-n)} .
$$

Here $A(k)$ is the number of admissible subsets with cardinality $k$, i.e. ${ }^{5}$

$$
A(k)=\sum_{k^{1}=\underline{\mu}^{1}}^{\bar{\mu}^{1}} \sum_{k^{2}=\underline{\mu}^{2}}^{\bar{\mu}^{2}} \cdots \sum_{k^{n-1}=\underline{\mu}^{n-1}}^{\bar{\mu}^{n-1}} \prod_{i=1}^{n}\left(\begin{array}{c}
\left|S^{i}\right| \\
k^{i}
\end{array}\right),
$$

where

$$
\begin{aligned}
& \underline{\mu}^{i}=\underline{\mu}^{i}\left(k^{1}, \ldots, k^{i-1}\right)=\max \left\{1 ; k-\sum_{j=1}^{i-1} k^{j}-\sum_{j=i+1}^{n}\left|S^{j}\right|\right\}, \\
& \bar{\mu}^{i}=\bar{\mu}^{i}\left(k^{1}, \ldots, k^{i-1}\right)=\min \left\{\left|S^{i}\right| ; k-\sum_{j=1}^{i-1} k^{j}-(n-i)\right\}, \quad \text { and } \\
& k^{n}=k^{n}\left(k^{1}, \ldots, k^{n-1}\right)=k-\sum_{j=1}^{n-1} k^{j} .
\end{aligned}
$$

Table 4.2 shows how the number of zeros increases when games become larger. For the same sizes, Table 4.1 showed an estimation of the mean number of Nash equilibria (see Table 9 of McLennan (1999)). Comparison of the two tables reveals that only a small fraction of the computed points are Nash equilibria.

Although it is known that for an open set of games with full Lebesgue measure the number of Nash equilibria is finite (see Harsanyi (1973)), it cannot be guaranteed that for the same set of games the set of (complex) solutions to $F^{D^{*}, u}(\delta)=0$ is finite for all admissible subsets $D^{*}$. In fact, up to now, there is no proof present that such a property holds. However, when the vector $u$ is allowed to be chosen from the complex space, the following holds true.

\footnotetext{
${ }^{5}$ Suppose we have $n$ vases where vase $i$ contains $\left|S^{i}\right|$ balls. The number $A(k)$ equals the number of possible outcomes when we pick $k$ balls with the condition that from each vase at least one ball must be picked.
} 


\begin{tabular}{cccccc}
\hline$n \backslash m$ & 2 & 3 & 4 & 5 & 6 \\
\hline 2 & 9 & 49 & 225 & 961 & 3969 \\
3 & 64 & 2197 & 64000 & 1771561 & 48228544 \\
4 & 625 & 194481 & 52200625 & $1.3521 \mathrm{e}+10$ & \\
5 & 7776 & 28629151 & $9.2390 \mathrm{e}+10$ & & \\
6 & 117649 & $6.3214 \mathrm{e}+09$ & & & \\
\hline
\end{tabular}

Table 4.2: Total number of solutions.

Theorem 4.2 For all admissible subsets $D^{*}$, there is an open set of vectors $u \in \mathbb{C}^{n|D|}$ with full Lebesgue measure such that the set of solutions to $F^{D^{*}, u}(\delta)=0$ is a compact zero-dimensional manifold.

Proof See Section 4.8 .

The set of vectors $u$ for which the property of the theorem above holds for all admissible subsets equals the intersection of the separate sets over the admissible subsets. Since the number of admissible subsets is finite, it concerns a finite intersection. More precisely, it is a finite intersection of open sets with full Lebesgue measure.

Theorem 4.3 There is an open set of vectors $u \in \mathbb{C}^{n|S|}$ with full Lebesgue measure such that for all admissible subsets $D^{*}$ the set of solutions to $F^{D^{*}, u}(\delta)=0$ is a compact zero-dimensional manifold.

Proof For an admissible subset $D^{*}$, let $\mathcal{U}\left(D^{*}\right)$ denote the open set of full Lebesgue measure from Theorem 4.2. Define $\mathcal{U}=\bigcap_{D^{*}} \overline{\mathcal{U}}\left(D^{*}\right)$, where $\overline{\mathcal{U}}\left(D^{*}\right)$ is the class of vectors $u \in \mathbb{C}^{n|S|}$ for which the natural restriction to $\mathbb{C}^{n|D|}, u_{\mid D^{*}}$, is in $\mathcal{U}\left(D^{*}\right)$. That the set $\mathcal{U}$ is an open set with full Lebesgue measure is obvious, as being a finite intersection of open sets with full Lebesgue measure.

This latter result seems rather weak since payoffs are commonly in real numbers, and the set $\mathbb{R}^{n|S|}$ is a set of measure zero in $\mathbb{C}^{n|S|}$. However, when a game with payoff-vector $u$ in real numbers is considered which is not in the generic set of the theorem, a small perturbation of the payoff-vector (if necessary, complex) suffices to obtain a payoff-vector which is. For this newly obtained payoff-vector it is possible to compute all candidate equilibria. Since the equilibrium correspondence is upper hemi-continuous, the candidate equilibria found are close to - and therefore good approximations of - the equilibria for the original game defined by the payoff-vector $u$. Because games are considered for which the number of equilibria is finite and for which the transversality conditions hold, locally (in the area of interest) the correspondence is 
also lower hemi-continuous, such that each equilibrium can be approximated by using complex perturbations.

\subsection{Implementation}

This section presents an algorithm to solve the systems of the previous section. The algorithm belongs to the class of homotopy-based algorithms. First, a general treatment of homotopy continuation methods to locate the zeros of a polynomial mapping is given. Next, one specific algorithm is discussed in detail, i.e. the algorithm used in the HOMPACK-routine POLSYS. Finally, the Gröbner basis is introduced as a method to transform the problem of solving a system of multivariate polynomials to the problem of (subsequently) solving single polynomial equations with one unknown.

\subsubsection{Homotopy continuation}

Many papers have been devoted on finding all solutions to a system $P$ of $n$ polynomial equations in $n$ unknowns using homotopy continuation ${ }^{6}$ methods on the only premises that the set of zeros is finite; see Chow, Mallet-Paret and Yorke (1979), Drexler (1977, 1978), Garcia and Li (1980), Garcia and Zangwill (1979a, 1979b, 1980), Kojima and Mizuno (1983), Mizuno (1981), Morgan (1983), Morgan and Sommese (1987), Morgan, Sommese and Watson (1989), Wright (1985), and Zulehner (1988).

A map $P: \mathbb{C}^{n} \rightarrow \mathbb{C}^{n}$ is polynomial if the maps $P_{k}: \mathbb{C}^{n} \rightarrow \mathbb{C}$ are polynomials for all $k=1, \ldots, n$, i.e. $P_{k}(z)$ is a sum of terms each of which has the form $a z_{1}^{b_{1}} z_{2}^{b_{2}} \cdots z_{n}^{b_{n}}$ for some $a \in \mathbb{C}$ and some nonnegative integers $b_{j}(j=1, \ldots, n)$. The sum of all $b_{j}$ s is the degree of the term, and the maximum of the degrees of the terms, $d_{k}$, is the degree of the polynomial $P_{k}$. The degree of $P$ is given by $d=\prod_{k=1}^{n} d_{k}$. Consider the system $P(z)=0$ of $n$ equations in $n$ unknowns. By Bezout's Theorem it follows that there are at most $d$ isolated solutions (if the number of solutions is finite).

Homotopy continuation methods can be used to find all the geometrically isolated solutions of $P(z)=0$. This works as follows. The system $P$ is embedded in a system of $n$ polynomial equations in $n+1$ unknowns where this new system includes the variables of $P$ and a new variable, the homotopy parameter. For one value of the homotopy parameter, the new system can be satisfactorily solved, and for another it is identical to $P$. The continuation process solves $P(z)=0$ by evolving or 'continuing' the full set of known solutions resulting for one value of the homotopy parameter into the full set of solutions to $P(z)=0$.

\footnotetext{
${ }^{6}$ Homotopy continuation is also called imbedding, continuation or incremental loading.
} 
The homotopy system is denoted by $H(t, z)=0$, where $H(1, z)=P(z)$ for all $z$ and the solutions to $H(0, z)=0$ are known. The homotopy parameter $t$ varies between 0 and 1 . The task is to continue the solutions of $H(0, z)=0$ into those of $H(1, z)=0$. Assuming sufficient conditions so that $H^{-1}(\{0\})$ consists of smooth paths, the continuation towards the solutions becomes a process of path tracking.

Many issues arise in attempting to implement this concept into a reliable and fast algorithm for computing all solutions to polynomial systems. Basically, there are two steps:

(1) Define the homotopy $H(t, z)$.

(2) Choose a numerical method for tracking the paths defined by $H(t, z)=0$.

The definition chosen in step (1) has to result in smooth paths in $H^{-1}(\{0\})$ which link the known solutions of $H(0, z)=0$ to the solutions of $P(z)=0$. More precisely, it has to be chosen such that the components of $H^{-1}(\{0\})$ topologically have the following properties:

1. A component may be a closed arc which intersects each slice $\{t\} \times \mathbb{C}^{n}, t \in[0,1]$, once. These components correspond to single roots of the system $P(z)=0$.

2. A component may consist of $m$ arcs which meet in a single point of $\{1\} \times \mathbb{C}^{n}$. This point is a root of the system $P(z)=0$ with multiplicity $m$. Each slice $\{t\} \times \mathbb{C}^{n}, t \in[0,1)$, will intersect such a component in $m$ points.

3. A component may be a half-open arc which intersects each slice $\{t\} \times \mathbb{C}^{n}, t \in[0,1)$, in a single point which tends to infinity as $t \rightarrow 1$. Such a component corresponds to an infinite root.

For the treatment of infinite roots there are two basic solutions: to define the homotopy such that for $t \in[0,1)$ the equations $H_{k}(t, z)=0$ are one degree higher than the equations $P_{k}(z)=0$, or to carry out the continuation in the complex projective space, a compactification of $\mathbb{C}^{n}$ which allows an explicit representation of infinite roots.

For step (2) there are two fundamental methods of numerically tracing those paths: predictorcorrector methods, and simplicial methods. Predictor-corrector methods approximately follow exact solution curves, whereas simplicial methods exactly follow approximate solution curves. For more theory on path-tracking methods the reader is referred to Allgower and Georg (1980, 1983, 1990, 1993) and Garcia and Zangwill (1981).

\subsubsection{Hompack}

HOMPACK (see Watson, Billups and Morgan (1987)) is a suite of codes that is programmed in FORTRAN and developed for following homotopy-paths numerically in order to compute fixed 
points or zeros. HOMPACK contains an algorithm, the so-called POLSYS-routine, which allows to solve completely for systems of polynomial equations on the only premises that the solution set is finite. Separate routines are provided for dense and sparse matrices. In Morgan, Sommese and Watson (1989) it is described how the POLSYS routine of the software package HOMPACK computes all isolated solutions of a polynomial system.

Consider a polynomial map $P: \mathbb{C}^{n} \rightarrow \mathbb{C}^{n}$. Define $Q: \mathbb{C}^{n} \rightarrow \mathbb{C}^{n}$ by

$$
Q_{k}(z)=\beta_{k} z_{k}^{d_{k}}-\alpha_{k}, \quad k=1, \ldots, n
$$

where $\alpha_{k}$ and $\beta_{k}$ are nonzero complex numbers, for $k=1, \ldots, n$. Define the homotopy map $H_{\alpha, \beta}:[0,1] \times \mathbb{C}^{n} \rightarrow \mathbb{C}^{n}$ by

$$
H_{\alpha, \beta}(t, z)=(1-t) Q(z)+t P(z),
$$

where $\alpha=\left(\alpha_{1}, \ldots, \alpha_{n}\right) \in \mathbb{C}^{n}$ and $\beta=\left(\beta_{1}, \ldots, \beta_{n}\right) \in \mathbb{C}^{n}$. The following result of Morgan (1987, p.124) applies.

Theorem 4.4 Given $P$, there are sets of measure zero, $A$ and $B$ in $\mathbb{C}^{n}$ such that, if $\alpha \notin A$ and $\beta \notin B$, then the following holds

1. The solution set $\left\{(t, z) \in[0,1) \times \mathbb{C}^{n} \mid H_{\alpha, \beta}(t, z)=0\right\}$ is a collection of d non-overlapping (smooth) paths;

2. The paths move from $t=0$ to $t=1$ without backtracking in $t$;

3. Each geometrically isolated solution of $P(z)=0$ of multiplicity $m$ has exactly $m$ continuation paths converging to it;

4. A continuation path can diverge to infinity only as $t \rightarrow 1$;

5. If $P(z)=0$ has no solutions at infinity, all the paths remain bounded. If $P(z)=0$ has a solution at infinity, at least one path will diverge to infinity as $t \rightarrow 1$. Each geometrically isolated solution at infinity of $P(z)=0$ of multiplicity $m$ will generate exactly $m$ diverging continuation paths.

For almost all choices of $\alpha$ and $\beta$ in $\mathbb{C}^{n}, H_{\alpha, \beta}^{-1}(\{0\})$ consists of $d$ smooth paths emanating from $\{0\} \times \mathbb{C}^{n}$, which either diverge to infinity as $t$ approaches 1 or converge to a solution to $P(z)=0$ as $t$ approaches 1 . And, each geometrically isolated solution of $P(z)=0$ has a path converging to it.

In HOMPACK, the algebraic context for generating the full solution list of a polynomial system is complex projective space rather than real or complex Euclidean space, thereby immediately providing a treatment for the infinite roots. For HOMPACK therefore the classical approach 
from algebraic geometry of homogenizing $P$ and establishing the continuation process in projective space is followed.

The complex projective space, $\mathbb{C P}^{n}$, consists of the lines through the origin in $\mathbb{C}^{n+1}$, denoted $\left[\left(z_{0}, \ldots, z_{n}\right)\right]$, where $\left(z_{0}, \ldots, z_{n}\right) \in \mathbb{C}^{n+1} \backslash\{0\}$; that is, $\left[\left(z_{0}, \ldots, z_{n}\right)\right]$ is the line through the origin that contains $\left(z_{0}, \ldots, z_{n}\right) . \mathbb{C P}^{n}$ can be seen as the disjoint union of points $\left[\left(z_{0}, \ldots, z_{n}\right)\right]$ with $z_{0} \neq 0$ identified with the Euclidean space via $\left[\left(z_{0}, \ldots, z_{n}\right)\right] \rightarrow\left(z_{1} / z_{0}, \ldots, z_{n} / z_{0}\right)$ and the 'points at infinity', the elements $\left[\left(z_{0}, \ldots, z_{n}\right)\right]$ with $z_{0}=0$.

Given $P_{k}\left(z_{1}, \ldots, z_{n}\right)$, let $P_{k}^{\perp}\left(z_{0}, \ldots, z_{n}\right)$ be defined as follows. Each term of $P_{k}^{\perp}$ is obtained from the corresponding term of $P_{k}$ by multiplying it by the power of $z_{0}$ to bring the degree of the term up to $d_{k}$. Thus, a term of $P_{k}$ of degree $\delta$ is multiplied by $z_{0}^{d_{k}-\delta}$, and consequently each term of $P_{k}^{\perp}$ has degree $d_{k}$. Thus, $P_{k}^{\perp}(\lambda z)=\lambda^{d_{k}} P_{k}^{\perp}(z)$, and $P_{k}^{\perp}$ takes all points of $\left[\left(z_{0}, \ldots, z_{n}\right)\right]$ to the same point. The map $P_{k}^{\perp}: \mathbb{C} \mathbb{P}^{n} \rightarrow \mathbb{C}$ is the homogenization of the map $P_{k}$. Then, $P^{\perp}$ - all $n$ components $P_{k}^{\perp}$ taken together - is a map form $\mathbb{C P}^{n}$ to $\mathbb{C}^{n}$. Note that if $P^{\perp}(z)=0$, then $P^{\perp}(\lambda z)=0$, for any non-zero complex scalar $\lambda$. Therefore, 'solutions' of $P^{\perp}(z)=0$ are (complex) lines through the origin.

The system $P^{\perp}\left(z_{0}, \ldots, z_{n}\right)=0$ reduces to the system $P(z)=0$ under the substitution $z_{0}=1$. Thus, the two systems can be considered to have the same set of roots in $\mathbb{C}^{n}$.

Theorem 4.5 There are no more than $d$ isolated solutions to $P^{\perp}(y)=0$ in $\mathbb{C P}^{n}$. If $P^{\perp}(y)=0$ has only a finite number of solutions in $\mathbb{C P}^{n}$, it has exactly d solutions, counting multiplicities.

To avoid dealing with $\mathbb{C P}^{n}$, a unique point is determined for each solution line. This point is $z \in \mathbb{C}^{n}$ such that either $(1, z)$ is on the solution line, or $(0, z)$ is and the first nonzero component of $z$ is 1 .

\subsubsection{Applying the Gröbner basis}

Let $\mathbb{C}\left[z_{1}, \ldots, z_{n}\right]$ denote the set of all polynomials in $n$ variables with coefficients in $\mathbb{C}$. For $p_{1}, \ldots, p_{s} \in \mathbb{C}\left[z_{1}, \ldots, z_{n}\right]$, the variety $V\left(p_{1}, \ldots, p_{s}\right)$ is defined to be the set of solutions of the system

$$
p_{1}=0, \ldots, p_{s}=0
$$

That is,

$$
V\left(p_{1}, \ldots, p_{s}\right)=\left\{\left(a_{1}, \ldots, a_{n}\right) \in \mathbb{C}^{n} \mid p_{i}\left(a_{1}, \ldots, a_{n}\right)=0, i=1, \ldots, s\right\} .
$$

The set $I=\left\langle p_{1}, \ldots, p_{s}\right\rangle=\left\{\sum_{i=1}^{s} u_{i} p_{i} \mid u_{i} \in \mathbb{C}\left[z_{1}, \ldots, z_{n}\right], i=1, \ldots, s\right\}$ is an ideal in $\mathbb{C}\left[z_{1}, \ldots, z_{n}\right]$; that is, if $p, q \in I$, then so is $p+q$ and if $p \in I$ and $r$ is any polynomial in $\mathbb{C}\left[z_{1}, \ldots, z_{n}\right]$, then $r p \in I$. The set $\left\{p_{1}, \ldots, p_{s}\right\}$ is called a generating set of the ideal $I$. According to Adams and Loustaunau (1994, p.3) the following holds. 
Theorem 4.6 The variety $V(I):=\left\{\left(a_{1}, \ldots, a_{n}\right) \in \mathbb{C}^{n} \mid p\left(a_{1}, \ldots, a_{n}\right)=0, p \in I\right\}$ is equal to the variety $V\left(p_{1}, \ldots, p_{s}\right)$. Or, stated differently, $p=0(p \in I)$ is equivalent to $p_{1}=0, \ldots, p_{s}=0$.

Now, if we have $I=\left\langle p_{1}, \ldots, p_{s}\right\rangle=\left\langle p_{1}^{\prime}, \ldots, p_{t}^{\prime}\right\rangle$, then $V\left(p_{1}, \ldots, p_{s}\right)=V(I)=V\left(p_{1}^{\prime}, \ldots, p_{t}^{\prime}\right)$. This means that the system $p_{1}=0, \ldots, p_{s}=0$ has the same solutions as the system $p_{1}^{\prime}=$ $0, \ldots, p_{t}^{\prime}=0$, and hence a variety is determined by an ideal, not by a particular set of equations. If we have a 'better' generating set for the ideal $I=\left\langle p_{1}, \ldots, p_{s}\right\rangle$, we will have a 'better' representation for the variety $V\left(p_{1}, \ldots, p_{s}\right)$. By 'better' is meant a set of generators that allows us to understand the algebraic structure of $I=\left\langle p_{1}, \ldots, p_{s}\right\rangle$ and the geometric structure of $V\left(p_{1}, \ldots, p_{s}\right)$ better. This 'better' generating set for $I$ is called a Gröbner basis for $I$ (see Gröbner $(1949,1970))$. In the case of linear polynomials this 'better' generating set is the one obtained from the row echelon form of the matrix in the system.

Applying Buchberger's algorithm, see Buchberger (1965), to a zero-dimensional ideal $I,{ }^{7}$ a typical Gröbner basis can be found, namely one in 'triangular' form (this is similar to the row echelon form in the linear case). Thus, in order to solve the system of equations determined by a zero-dimensional ideal $I$, it suffices to have an algorithm to find the roots of polynomials in one variable. That is, first the equation in one variable, $q_{1}\left(z_{1}\right)=0$, is solved. Subsequently, for each solution $\alpha$ of $q_{1}\left(z_{1}\right)=0$, the equation $q_{2}\left(\alpha, z_{2}\right)=0$ is solved. The computation process continues in this manner all the way until $q_{n}\left(\alpha_{1}, \ldots, \alpha_{n-1}, z_{n}\right)=0$ is solved. The solutions obtained in this way are the only possible solutions. Finally the solutions are tested by equations $q_{n+1}=0, \ldots, q_{t}=0$ (in the case when $t>n$ ) in order to obtain the set of solutions of the full system of equations. For a thorough introduction into Gröbner bases the reader is referred to Adams and Loustaunau (1994) and Cox, Little, and O'Shea (1996).

An important advantage of having the triangular structure is that the problem of finding all zeros of a system of polynomials can be reduced to repeatedly finding all zeros of a single polynomial. Each step the zeros that are not finite, nonnegative and real can be filtered out, which increases performance of the algorithm in terms of computation time. A disadvantage of the application of the Gröbner basis is that the degree of the 'new' system may increase enormously, since the number of polynomials as well as the degree of each polynomial separately may increase.

\subsection{Description of algorithm}

In this section the proposed algorithm to compute all equilibria is comprehensively described in a step-wise manner.

\footnotetext{
${ }^{7}$ Since the field of concern is the complex space and given the fact that this space is algebraically closed, saying that the ideal $I$ is zero-dimensional is equivalent to saying that the variety $V(I)$ is finite.
} 
Step 1. In the first step of the algorithm, the game is decomposed by means of support-sets. Each decomposition can be seen as a game for which the strategy set of each player is restricted as only to use strategies that belong to the support-set.

Step 2. When the support-restricted game has a special structure - for instance, when only two players have more than one strategy such that the support-restricted game is equivalent to a bimatrix game - it is possible to use existing algorithms especially developed for the class of games having that structure. In this case we can forward the found candidate equilibria to the final step of the algorithm. Otherwise a set of polynomial equations is formulated for which the set of solutions contain all completely mixed equilibria of the support-restricted game.

Step 3. The third step of the algorithm is optional. Here Buchberger's algorithm can be applied to deform the system of polynomial equations into one with a special structure, i.e. in 'triangular form'.

Step 4. In this step the bulk of the computations are done by using a numerical method to compute all solutions of the system of polynomial equations. The POLSYS-routine of HOMPACK is used to do these computations. When Buchberger's algorithm is applied it suffices to use a numerical algorithm to find all solutions to a single polynomial equation subsequently. In both cases the found candidate equilibria are forwarded to the next step.

Step 5. The candidate equilibria found are checked whether they really are equilibria of the original game. The solutions that are not nonnegative and real are removed as are the solutions for which there is a player that can obtain a better payoff by using a strategy that is outside the support to which it is temporarily restricted (for computational purposes).

\subsection{An alternative method}

In this section an alternative method is presented, one for which no decomposition by means of carriers is needed. Here, the set of Nash equilibria is computed by computing all zeros of only one system of polynomials. From Herings and Peeters (2001a) or from Chapter 3 it follows that a strategy-tuple $\sigma$ is an equilibrium with equilibrium payoffs $\mu$ if and only if it is part of a solution to

$$
\begin{aligned}
& u^{i}\left(\sigma^{-i}, s_{j}^{i}\right)+\lambda_{j}^{i}-\mu^{i}=0, \quad\left(s_{j}^{i} \in S^{i}, i \in N\right), \\
& \sum_{s_{j}^{i} \in S^{i}} \sigma_{j}^{i}-1=0, \quad(i \in N), \\
& \sigma_{j}^{i} \geq 0, \quad \lambda_{j}^{i} \geq 0, \quad \sigma_{j}^{i} \lambda_{j}^{i}=0, \quad\left(s_{j}^{i} \in S^{i}, i \in N\right) .
\end{aligned}
$$

If the set of equations

$$
\begin{aligned}
& u^{i}\left(\sigma^{-i}, s_{j}^{i}\right)+\lambda_{j}^{i}-\mu^{i}=0, \quad\left(s_{j}^{i} \in S^{i}, i \in N\right), \\
& \sum_{s_{j}^{i} \in S^{i}} \sigma_{j}^{i}-1=0, \quad(i \in N),
\end{aligned}
$$




$$
\sigma_{j}^{i} \lambda_{j}^{i}=0, \quad\left(s_{j}^{i} \in S^{i}, i \in N\right),
$$

is solved, and solutions for which some component of $\lambda$ or $\sigma$ is not a finite nonnegative real are filtered out, the set of Nash equilibria is what remains. The previous system of equations is equivalent to

$$
\begin{aligned}
& \sigma_{j}^{i} u^{i}\left(\sigma^{-i}, s_{j}^{i}\right)-\sigma_{j}^{i} \mu^{i}=0, \quad\left(s_{j}^{i} \in S^{i}, i \in N\right), \\
& \sum_{s_{j}^{i} \in S^{i}} \sigma_{j}^{i}-1=0, \quad(i \in N) .
\end{aligned}
$$

Now, filtering for $\sigma_{j}^{i}$ and $\mu^{i}-u^{i}\left(\sigma^{-i}, s_{j}^{i}\right)$ to be finite nonnegative reals for all $s_{j}^{i} \in S^{i}$ and all $i \in N$ is required. All remaining solutions together form the complete set of Nash equilibria.

Like in the original method, it is necessary to go to the complex space for being sure of having found all solutions to the system of polynomial (multilinear) equations. Here, in contrast to the original method, the problem is not decomposed by means of carriers (admissible subsets). So, when using this method, only one system of equations needs to be solved. Although this seems to be an advantage, the system has also the $\mu \mathrm{s}$ as variables and therefore the system is one of higher dimension. It is not clear, therefore, which system is more efficient in terms of computation time.

\subsection{Summary}

In this chapter a method is described to find all Nash equilibria of an $n$-person normal form game. Several techniques are mentioned to speed up the computation times.

First, for bimatrix games existing algorithms can be applied which might be faster. These algorithms can also be applied for certain carrier decompositions. Decomposition allows also for parallel computation, since the problem can be divided into a finite number of small(er) problems.

Secondly, it is possible to apply Buchberger's algorithm in order to transform the problem of finding all solutions to a system of polynomial equations in subsequently finding all solutions to single polynomial equations. When for one polynomial a zero is found, it is substituted in the next polynomial to solve. Therefore, again it is possible to apply parallel computers.

Finally, there is no need to wait until the algorithm has finally converged. It finds more and more Nash equilibria while running, and can therefore also be useful where the sheer size of the problem makes it practically impossible to really find all equilibria.

\subsection{Proofs}

To prove Theorem 4.2, the following lemma is needed. 
Lemma 4.7 For all admissible subsets $D^{*}$, there is an open set of vectors $u \in \mathbb{C}^{n|S|}$ with full Lebesgue measure such that the Jacobian of $F^{D^{*}, u}$ has full rank in its zero points.

Proof Let an admissible subset $D^{*}$ of $S^{*}$ and a vector $u \in \mathbb{C}^{n|S|}$ be given and let the function $F^{D^{*}}: \mathbb{C}^{\left|D^{*}\right|} \times \mathbb{C}^{n|D|} \rightarrow \mathbb{C}^{\left|D^{*}\right|}$ be defined such that $F^{D^{*}}(\delta, u)=F^{D^{*}, u}(\delta)$. If $\partial_{(\delta, u)} F^{D^{*}}(\bar{\delta}, \bar{u})$, the Jacobian of $F^{D^{*}}$ in $(\bar{\delta}, \bar{u})$, has full rank for all $(\bar{\delta}, \bar{u})$ such that $F^{D^{*}}(\bar{\delta}, \bar{u})=0$, then it follows by the transversality theorem, Theorem 2.26, that $\partial_{\delta} F^{D^{*}, u}(\bar{\delta})$, the Jacobian of $F^{D^{*}, u}$ in $\bar{\delta}$, has full rank for all $\bar{\delta}$ such that $F^{D^{*}, u}(\bar{\delta})=0$ except for a set of vectors $u$ with zero Lebesgue measure. So, we have to prove that $\partial_{(\delta, u)} F^{D^{*}}$ has full rank in points $(\bar{\delta}, \bar{u})$ such that $F^{D^{*}}(\bar{\delta}, \bar{u})=0$.

It is easily seen that

$$
\begin{aligned}
& \partial_{\delta_{\ell}^{k}}\left(\sum_{d_{j}^{i} \in D^{i}} \delta_{j}^{i}-1\right)=\mathbb{1}_{i=k}, \\
& \partial_{u^{k}\left(\underline{d}^{-k}, d_{\ell}^{k}\right)}\left(\sum_{d_{j}^{i} \in D^{i}} \delta_{j}^{i}-1\right)=0,
\end{aligned}
$$

where 11 represents the identity function that assigns value one if the condition in the subscript is satisfied and zero otherwise, and that

$$
\begin{aligned}
& \partial_{\delta_{\ell}^{k}}\left(u^{i}\left(\delta^{-i}, \tilde{d}^{i}\right)-u^{i}\left(\delta^{-i}, d_{j}^{i}\right)\right)=\left(u^{i}\left(\delta^{-i, k}, d_{\ell}^{k}, \tilde{d}^{i}\right)-u^{i}\left(\delta^{-i, k}, d_{\ell}^{k}, d_{j}^{i}\right)\right) \cdot \mathbb{1}_{i \neq k}, \\
& \partial_{u^{k}\left(\underline{d}^{-k}, d_{\ell}^{k}\right)}\left(u^{i}\left(\delta^{-i}, \tilde{d}^{i}\right)-u^{i}\left(\delta^{-i}, d_{j}^{i}\right)\right)=\delta\left(\underline{d}^{-i}\right) \cdot\left(\mathbb{1}_{d_{\ell}^{k}=\tilde{d}^{i}}-\mathbb{1}_{d_{\ell}^{k}=d_{j}^{i}}\right) .
\end{aligned}
$$

Further we know that

$$
\sum_{d^{-i} \in D^{-i}} \delta\left(d^{-i}\right)=1,
$$

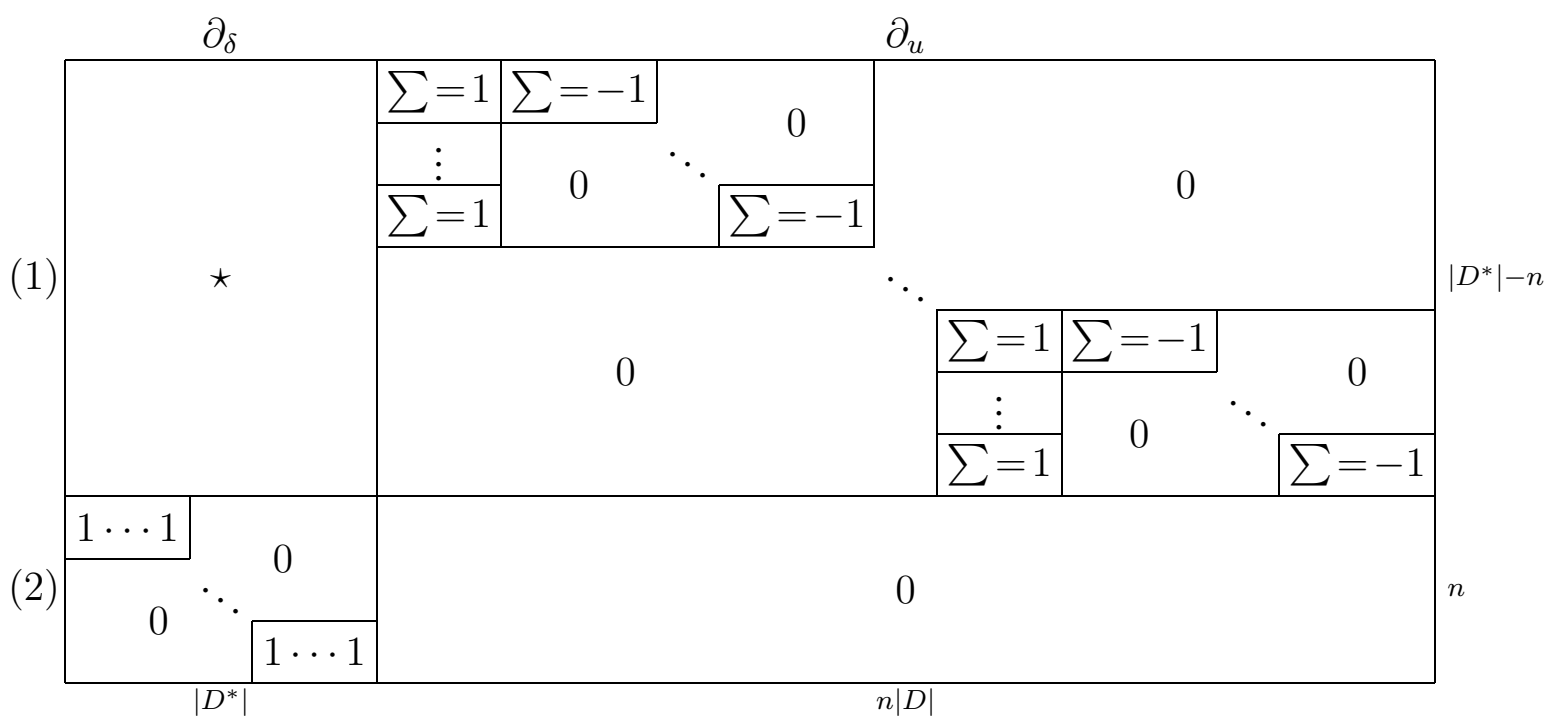

Figure 4.1: Jacobian of $F^{D^{*}}$. 
and therefore

$$
\sum_{d^{-i} \in D^{-i}} \partial_{u^{k}\left(d^{-k}, d_{\ell}^{k}\right)}\left(u^{i}\left(\delta^{-i}, \tilde{d}^{i}\right)-u^{i}\left(\delta^{-i}, d_{j}^{i}\right)\right)=\mathbb{1}_{d_{\ell}^{k}=\tilde{d}^{i}}-\mathbb{1}_{d_{\ell}^{k}=d_{j}^{i}} .
$$

So, the Jacobian of $F^{D^{*}}$ in points $(\bar{\delta}, \bar{u})$ for which it holds that $F^{D^{*}}(\bar{\delta}, \bar{u})=0$ has the form as depicted in Figure 4.1 (for certain ordering of variables and equations). In this figure the box containing the star is not specified because it is not necessary to know that part of the matrix to see that the matrix has full rank.

Proof of Theorem 4.2 From Lemma 4.7, it follows that for any admissible subset $D^{*}$ and for almost every vector $u$, the set $\left\{\delta \in \mathbb{C}^{\left|D^{*}\right|} \mid F^{D^{*}, u}(\delta)=0\right\}$ is a regular constraint set (see Section 2.4). By counting the number of equations and variables it follows that $\left\{\delta \in \mathbb{C}^{\left|D^{*}\right|} \mid F^{D^{*}, u}(\delta)=0\right\}$ is a zero-dimensional manifold. Compactness is obvious. 


\section{Part II}

\section{Stochastic Games}





\section{Chapter 5}

\section{Equilibrium Selection}

In this chapter, which is based on Herings and Peeters (2001b), a selection theory for stochastic games is developed. The theory itself is based on the ideas of Harsanyi and Selten to select equilibria for games in standard form. First several possible definitions for the stochastic tracing procedure, an extension of the linear tracing procedure to the class of stochastic games, are introduced. Then the properties of these alternative definitions are analyzed. Finally, it is shown that exactly one of the proposed extensions is consistent with the formulation of Harsanyi-Selten for games in standard form and captures stationarity.

\subsection{Introduction}

Stochastic games were introduced by Shapley (1953). He considered both finite and infinite horizon two-person zero-sum stochastic games with finite state space and finite action spaces. Shapley proved that such games have a value and that both players have optimal stationary strategies with respect to the discounted payoff criterion. Fink (1964), Takahashi (1964), and Sobel (1971) extended Shapley's model to $n$-person non-zero-sum stochastic games. For the model with finite state space and finite action spaces they showed the existence of a stationary equilibrium.

A stochastic game is played in stages. At each stage, the game is in one of finitely many states and every player observes the current state. In each stage the players have to make a choice (simultaneously and independently) out of the action sets which depend on the state. These choices result not only in a payoff (each state is coupled with a normal form game), but also in an action dependent probability measure on the set of states. Next, according to this probability measure, a chance experiment is carried out to determine the state of the next stage.

For many normal form games there is a vast multiplicity of equilibria, see McLennan (1999). There is no reason to expect that the situation is different for the multiplicity of equilibria in stochastic games. Even though such a game may possess many non-stationary equilibria, there are good reasons to focus on stationary equilibria. But, concerning the multiplicity of stationary 
equilibria there is no reason to expect that this number is small. After all, when the dynamics does not allow to jump to states other than the initial one, for instance, the stochastic game is equivalent to a finite number of repeated games, and the set of stationary equilibria equals the Cartesian product of the Nash equilibria of the separate stage games. It follows that there are many stationary equilibria. Therefore, it is essential to provide a selection theory that selects a particular stationary equilibrium.

In Harsanyi and Selten (1988) the tracing procedure is defined for normal form games and for extensive form games with a perfect recall information structure (in these games at every point every player remembers whatever he knew in the past). For extensive form games, Harsanyi and Selten (1988) first transform the game into one in standard form and subsequently define the tracing procedure for that class of games. Since the expected utility property does not hold in stochastic games, it is not possible to transform a stochastic game into one in standard form. The way to extend the tracing procedure to the class of stochastic games is neither straightforward nor unique.

In this chapter a variant of the tracing procedure which allows selection within the class of stationary equilibria of stochastic games is introduced. Thereto, four ways to extend the linear tracing procedure of Harsanyi and Selten (1988) to the setting of stochastic games in a natural way are presented. The four alternatives originate from two choices of modeling beliefs in stochastic games. First, there is the assumption about beliefs within states. Second, there is the assumption about beliefs across time (stages). Both belief-types might be assumed to be correlated or not. After properly defining the four alternatives, they are analyzed. It is found that the four alternatives are essentially different, in the sense that they may select different equilibria. Only one of the extensions turns out to be consistent with the linear tracing procedure while capturing stationarity. This extension of the linear tracing procedure to the class of stochastic games is labeled as the stochastic tracing procedure.

This chapter is organized as follows. In Section 5.2 the finite discounted stochastic game model in described. Further, the restriction to stationary strategies is made, and the concept of stationary equilibrium is defined. Section 5.3 comprehensively defines the linear tracing procedure and exposes some of its properties. In Section 5.4, the four extensions of the linear tracing procedure to the class of stochastic games are proposed and defined. Section 5.5 analyzes the four alternatives. The last section, Section 5.6, summarizes comprehensively.

\subsection{Finite discounted stochastic games}

In this chapter finite discounted stochastic games are studied. A finite discounted stochastic game is an ordered sextuple

$$
\Gamma=\left\langle N, \Omega,\left\{S_{\omega}^{i}\right\}_{(i, \omega) \in N \times \Omega},\left\{u^{i}\right\}_{i \in N}, \pi, \delta\right\rangle,
$$


where $N, \Omega$ and $S_{\omega}^{i}$ are finite non-empty sets, $S_{\omega}=\chi_{i \in N} S_{\omega}^{i}, u^{i}$ is a real-valued function on the set $H=\left\{\left(\omega, s_{\omega}\right) \mid \omega \in \Omega, s_{\omega} \in S_{\omega}\right\}, \pi$ is a map $\pi: H \rightarrow \Delta(\Omega)$ with $\Delta(\Omega)$ the family of probability distributions on the space $\Omega$, and $\delta$ is a real number. The game parameters have the following meaning.

- $N=\{1, \ldots, n\}$ is the player set.

- $\Omega=\left\{\omega_{1}, \ldots, \omega_{z}\right\}$ is the state space.

- $S_{\omega}^{i}=\left\{s_{\omega 1}^{i}, \ldots, s_{\omega m_{\omega}^{i}}^{i}\right\}$ is the action set of player $i \in N$ in state $\omega \in \Omega$.

- $u^{i}: H \rightarrow \mathbb{R}$ is the payoff function of player $i \in N$; if in state $\omega \in \Omega$ the players action choices are $s_{\omega}=\left(s_{\omega}^{1}, \ldots, s_{\omega}^{n}\right) \in S_{\omega}$, then player $i$ gets an instantaneous payoff of $u^{i}\left(\omega, s_{\omega}\right)$.

- $\pi: H \rightarrow \Delta(\Omega)$ is the transition map. For each $\left(\omega, s_{\omega}\right) \in H, \pi\left(\omega, s_{\omega}\right)$ is identified with the vector $\left(\pi\left(\omega_{1} \mid \omega, s_{\omega}\right), \ldots, \pi\left(\omega_{z} \mid \omega, s_{\omega}\right)\right)$. Here $\pi\left(\bar{\omega} \mid \omega, s_{\omega}\right)$ represents the probability that the system jumps to state $\bar{\omega}$ if in state $\omega$ the strategy-tuple $s_{\omega}$ is played. Hence, $\pi\left(\bar{\omega} \mid \omega, s_{\omega}\right) \geq 0$ and $\sum_{\bar{\omega} \in \Omega} \pi\left(\bar{\omega} \mid \omega, s_{\omega}\right)=1$.

- $\delta \in(0,1)$ is the discount factor and is used to discount future payoffs.

Such a stochastic game corresponds to a dynamic system which can be in different states and where at certain stages the players can influence the course of the play. Here, the infinite horizon model is considered and the set of stages is represented by the set $\mathbb{Z}_{+}=\{0,1, \ldots\}$. Players know the game itself and this knowledge is common knowledge among all the players. Moreover, the initial state $\omega^{0} \in \Omega$ at stage $k=0$ is common knowledge to the players.

The game proceeds as follows. All players $i$ select at the initial state $\omega^{0}$, simultaneously and independently of each other (possibly by a chance experiment), an action $s_{\omega^{0}}^{i} \in S_{\omega^{0}}^{i}$. Now two things happen, both depending on the current state $\omega^{0}$ and the action choices $s_{\omega^{0}}^{1}, \ldots, s_{\omega^{0}}^{n}$.

(a) Player $i$ earns $u^{i}\left(\omega^{0}, s_{\omega^{0}}\right)$.

(b) The system jumps to the next state $\omega^{1}$ according to the outcome of a chance experiment. The probability that the next state will be $\bar{\omega}$ equals $\pi\left(\bar{\omega} \mid \omega^{0}, s_{\omega^{0}}\right)$.

Subsequently, prior to the next stage $k=1$, all players are informed about the previous actions chosen by the players, and of the new state $\omega^{1}$. At stage $k=1$, the above procedure is repeated, starting from the state $\omega^{1}$. This procedure goes on and on ad infinitum.

The game is assumed to be of perfect recall, i.e. at each stage each player remembers all past actions chosen by all players and all past states that have occurred. Note that for finite stochastic games, each stage game resembles a normal form game. However, contrary to the situation 
with normal form games, the game does not consist of a single play, but jumps according to the probability measure $\pi\left(\cdot \mid \omega, s_{\omega}\right)$ to the next state and continues dynamically. In choosing an action in a certain state, a player not only takes into account the immediate payoff, but also his opportunities in future states.

Like in normal form games, the players are allowed to randomize their pure actions. A mixed strategy of player $i$ in state $\omega$ is a probability distribution on $S_{\omega}^{i}$. The set of all probability distributions on $S_{\omega}^{i}$ is identified with $\Sigma_{\omega}^{i}$. For $\sigma_{\omega}^{i} \in \Sigma_{\omega}^{i}$, the probability assigned to pure strategy $s_{\omega j}^{i}$ is given by $\sigma_{\omega j}^{i}$. The strategy space of the normal form game in state $\omega$ is therefore equal to $\Sigma_{\omega}=X_{i \in N} \Sigma_{\omega}^{i}$. Given a mixed strategy combination $\sigma_{\omega} \in \Sigma_{\omega}$ and a strategy $\bar{\sigma}_{\omega}^{i} \in \Sigma_{\omega}^{i},\left(\sigma_{\omega}^{-i}, \bar{\sigma}_{\omega}^{i}\right)$ denotes the mixed strategy that results from replacing $\sigma_{\omega}^{i}$ by $\bar{\sigma}_{\omega}^{i}$. If a mixed strategy combination $\sigma_{\omega} \in \Sigma_{\omega}$ is played, then the instantaneous expected payoff of player $i$ is denoted by $u^{i}\left(\omega, \sigma_{\omega}\right)$ and the expected transition to state $\bar{\omega}$ by $\pi\left(\bar{\omega} \mid \omega, \sigma_{\omega}\right)$.

At the different stages this randomization may depend on the history of the game up to that stage. A history up to a stage $k$ is a sequence $h^{k}=\left(\omega^{0}, s_{\omega^{0}}, \omega^{1}, s_{\omega^{1}}, \ldots, \omega^{k-1}, s_{\omega^{k-1}}\right)$ that could have occurred up to time $k, k \geq 1$. Here, $\omega^{\kappa}$ represents the state and $s_{\omega^{\kappa}}$ the actions of the players at stage $\kappa, \kappa=0, \ldots, k-1$. Obviously, the set of histories up to time $k$ equals $H^{k}=X_{\kappa=0}^{k-1} H$, i.e. the $k$-fold Cartesian product of $H$.

A behavior strategy $\sigma^{i k}$ of player $i$ specifies for each stage $k$, each state $\omega^{k}$ at time $k$, and each history $h^{k}$ a probability distribution $\sigma^{i k}\left(h^{k}, \omega^{k}\right)$ on the action space $S_{\omega^{k}}^{i}$ of player $i$ in state $\omega^{k}$. Then $\sigma^{i k}\left(s_{\omega^{k}}^{i} \mid h^{k}, \omega^{k}\right)$ is the probability with which player $i$ chooses action $s_{\omega^{k}}^{i} \in S_{\omega^{k}}^{i}$ at time $k$ if state $\omega^{k}$ and history $h^{k}$ have occurred. So, a behavior strategy $\sigma^{i}$ for player $i$ is a sequence $\left(\sigma^{i 0}, \sigma^{i 1}, \ldots\right)$ where $\sigma^{i 0} \in \Sigma^{i}:=\chi_{\omega \in \Omega} \Sigma_{\omega}^{i}$ and $\sigma^{i k}: H^{k} \rightarrow \Sigma^{i}$ for all $k \geq 1$.

Given initial state $\omega \in \Omega$ and strategy-tuple $\sigma=\left(\sigma^{1}, \ldots, \sigma^{n}\right)$, player $i$ evaluates the stream of expected payoffs by

$$
U^{i}(\omega, \sigma):=\sum_{k=0}^{\infty} \delta^{k} \cdot U^{i k}(\omega, \sigma)
$$

where $U^{i k}(\omega, \sigma)$ denotes the expected instantaneous utility at stage $k$. Here, $U^{i}(\omega, \sigma)$ equals the total discounted expected payoff of player $i$ when the discount factor equals $\delta$, the starting state is $\omega$ and the strategy-tuple $\sigma$ is played. Since the state and action spaces are assumed to be finite, $U^{i}(\omega, \sigma)$ exists.

A strategy-tuple $\sigma$ is an equilibrium if and only if $\sigma^{i}$ is a best response to $\sigma^{-i}$ for all $i \in N$. If $n=2$ and the payoffs are zero-sum, then the definition of an equilibrium characterizes a minimax solution (see Shapley (1953)).

A stationary strategy for player $i$ is a behavior strategy for which $\sigma^{i k}\left(h^{k}, \omega^{k}\right)$ is of the form $\sigma^{i}\left(\omega^{k}\right)$, i.e. a stationary strategy is a history and time independent strategy which depends on the state only. In the sequel, a stationary strategy for player $i$ will be denoted by the symbol $\rho^{i}$. Define $\rho^{i}=\left(\rho_{\omega}^{i}\right)_{\omega \in \Omega}$, where $\rho_{\omega}^{i}$ is a probability measure on the action space $S_{\omega}^{i}$ for each $\omega \in \Omega$. 
So $\rho_{\omega}^{i} \in \Sigma_{\omega}^{i}$. If player $i$ decides to play the stationary strategy $\rho^{i}$, then every time that the system is in state $\omega$, player $i$ selects his pure action according to $\rho_{\omega}^{i}$. So, a stationary strategy $\rho^{i}$ for player $i$ is an element of $\Sigma^{i}$.

In the sequel of this chapter the analysis is restricted to stationary strategies. ${ }^{1}$ Several motivations for analyzing stationary equilibria can be found in Maskin and Tirole (2001). Stationary strategies prescribe the simplest form of behavior that is consistent with rationality, stationarity captures the notion that 'bygones are bygones' more completely than does the concept of subgame-perfect equilibrium, and it embodies the principle that 'minor causes should have minor effects', that is, only those aspects of the past that are 'significant' should have an appreciable influence on behavior. The pragmatic motivations they give are that in applied theory, the focus on stationary strategies allows for clean, unobstructed analysis of the influence of the state variables, that stationary strategies substantially reduce the number of parameters to be estimated in dynamic (econometric) models, and that stationary models can be simulated.

Suppose that $\omega$ is the initial state and the players decide to play a stationary strategy-tuple $\rho$. The total discounted expected payoff of player $i$ is denoted by $U^{i}(\omega, \rho)$. The instantaneous payoff player $i$ obtains in stage $k=0$ equals $u^{i}\left(\omega, \rho_{\omega}\right)$. The probability that at the next stage the state will be $\bar{\omega}$ equals $\pi\left(\bar{\omega} \mid \omega, \rho_{\omega}\right)$.

When $\rho$ is a stationary strategy-tuple and $\omega$ is the initial state, the expected payoffs are given by the following recursive formula (see e.g. Fink (1964))

$$
U^{i}(\omega, \rho)=u^{i}\left(\omega, \rho_{\omega}\right)+\delta \cdot \sum_{\bar{\omega} \in \Omega} \pi\left(\bar{\omega} \mid \omega, \rho_{\omega}\right) U^{i}(\bar{\omega}, \rho) .
$$

A stationary strategy-combination $\rho \in \Sigma$ is a stationary equilibrium if it is a Nash equilibrium in stationary strategies. It is a very well-known result that there exists a stationary best response to stationary strategies $\rho^{-i}$. Therefore, a stationary strategy-combination $\bar{\rho} \in \Sigma$ is a stationary equilibrium if for all $i$ and all $\omega$

$$
U^{i}(\omega, \bar{\rho}) \geq U^{i}\left(\omega, \bar{\rho}^{-i}, \rho^{i}\right), \quad\left(\rho^{i} \in \Sigma^{i}\right) .
$$

Another famous result, see Fink (1964), Takahashi (1964), or Sobel (1971), is that for every finite discounted stochastic game a stationary equilibrium exists. In Haller and Lagonoff (2000) it is shown that the number of stationary equilibria is generically finite. In Herings and Peeters (2000) this result is sharpened to generic oddness (see also Chapter 6). For $\delta=0$, the number of stationary equilibria is the product of the number of Nash equilibria of the separate stage games. It is shown in McLennan (1999) that the number of Nash equilibria of each stage game might be enormous. So, for $\delta=0$ the number of stationary equilibria is enormous. There is no reason to expect that the number of stationary equilibria will not be huge when $\delta$ is positive.

\footnotetext{
${ }^{1}$ In fact, restrict is made to a class which is even tighter. Namely, the one of perfect stationary strategies, where the strategy chosen is as if each state is reached or might be the initial state.
} 


\subsection{Linear tracing procedure}

In this section, a state $\omega$ is fixed and the linear tracing procedure for the corresponding normal form game $\Gamma_{\omega}=\left\langle N,\left\{S_{\omega}^{i}\right\}_{i \in N},\left\{u^{i}(\omega)\right\}_{i \in N}\right\rangle$ is briefly explained. A more extensive introduction to the linear tracing procedure is provided in Chapter 3.

The linear tracing procedure is a mathematical construct to model a process of convergent expectations, by which rational players come to adopt, and expect each other to adopt, one particular equilibrium point as the outcome for a given game. At the beginning of this outcome-selection process, the players will as yet lack any specific theory predicting the strategies to be used by the other players. Accordingly, each player will express his expectations about the strategy choice of any other player in the form of a subjective probability distribution over the other players' pure strategies. These subjective distributions are called prior probability distributions, or simply priors. An important assumption of this model is that all players other than $i$ will associate the same prior probability distribution $p_{\omega}^{i}$ with any given player $i$. Part of the Harsanyi-Selten theory is the determination of a prior.

The linear tracing procedure is based on a one-parameter family of auxiliary games $\Gamma_{\omega}^{t}$ with $t \in[0,1]$. In any game $\Gamma_{\omega}^{t}$, every player $i$ has the same strategy set $\Sigma_{\omega}^{i}$ as he has in the original game $\Gamma_{\omega}$. But his payoff function $v^{i}(t ; \omega)$ in $\Gamma_{\omega}^{t}$ is

$$
v^{i}\left(t ; \omega, \rho_{\omega}\right)=(1-t) u^{i}\left(\omega, p_{\omega}^{-i}, \rho_{\omega}^{i}\right)+t u^{i}\left(\omega, \rho_{\omega}\right)
$$

where $u^{i}(\omega)$ is his payoff in the original game $\Gamma_{\omega}$. Clearly, $v^{i}\left(1 ; \omega, \rho_{\omega}\right)=u^{i}\left(\omega, \rho_{\omega}\right)$ so that $\Gamma_{\omega}^{1}=\Gamma_{\omega}$. On the other hand, $v^{i}\left(0 ; \omega, \rho_{\omega}\right)=u^{i}\left(\omega, p_{\omega}^{-i}, \rho_{\omega}^{i}\right)$.

Thus $\Gamma_{\omega}^{0}$ is a game of a rather special structure in which the payoff $v^{i}(0 ; \omega)$ of each player $i$ will depend only on his own strategy $\rho_{\omega}^{i}$ and will be independent of the other players' strategycombination $\rho_{\omega}^{-i}$. Consequently, the game $\Gamma_{\omega}^{0}$ naturally decomposes into several mutually independent and separate maximization problems, one for each player. For almost all games $\Gamma_{\omega}$, for almost all choices of the prior vector $p_{\omega}$, the game $\Gamma_{\omega}^{0}$ has exactly one equilibrium point $\rho_{\omega}^{0}$, which is in pure strategies.

For any auxiliary game $\Gamma_{\omega}^{t}$, the set of all equilibrium points in $\Gamma_{\omega}^{t}$ is denoted by $\mathcal{E}\left(\Gamma_{\omega}^{t}\right)$. By Nash's (1951) existence theorem for equilibrium points, all of these sets are nonempty. Let $\mathcal{L}_{\omega}=\mathcal{L}\left(\Gamma_{\omega}, p_{\omega}\right)$ be the graph of the correspondence $t \rightarrow \mathcal{E}\left(\Gamma_{\omega}^{t}\right)$ for $t \in[0,1] . \mathcal{L}_{\omega}$ is typically a collection of pieces of one-dimensional algebraic curves, though in degenerate cases it may also contain isolated points and/or subsets of more than one dimension, see Chapter 3.

Suppose the graph $\mathcal{L}_{\omega}$ contains a path $\gamma_{\omega}$ connecting a point $x_{\omega}^{0}=\left(0, \rho_{\omega}^{0}\right)$, corresponding to an equilibrium point $\rho_{\omega}^{0}$ of the game $\Gamma_{\omega}^{0}$, with a point $x_{\omega}^{1}=\left(1, \rho_{\omega}^{*}\right)$, corresponding to an equilibrium point $\rho_{\omega}^{*}$ of the original game $\Gamma_{\omega}^{1}=\Gamma_{\omega}$. Then $\gamma_{\omega}$ is called a feasible path, whereas $x_{\omega}^{0}$ and $x_{\omega}^{1}$ are called the starting point and the end point of this path $\gamma_{\omega}$, respectively. Moreover, 
the strategy part $\rho_{\omega}^{*}$ of this end point $x_{\omega}^{1}$ is called the outcome selected by the path $\gamma_{\omega}$. This strategy combination $\rho_{\omega}^{*}$ can be rationally selected as the outcome of the game because it always is an equilibrium point of the original game $\Gamma_{\omega}$.

The linear tracing procedure consists of selecting an outcome $\rho_{\omega}^{*}$ for any game $\Gamma_{\omega}$ by tracing a feasible path $\gamma_{\omega}$ from its starting point $x_{\omega}^{0}=\left(0, \rho_{\omega}^{0}\right)$ to its end point $x_{\omega}^{1}=\left(1, \rho_{\omega}^{*}\right)$. For any given pair $\left(\Gamma_{\omega}, p_{\omega}\right)$, the linear tracing procedure is called feasible if the graph $\mathcal{L}_{\omega}=\mathcal{L}\left(\Gamma_{\omega}, p_{\omega}\right)$ contains at least one feasible path $\gamma_{\omega}$, and is called well-defined if $\mathcal{L}_{\omega}$ contains exactly one feasible path $\gamma_{\omega}$.

For any possible pair $\left(\Gamma_{\omega}, p_{\omega}\right)$, the linear tracing procedure is always feasible but is not always well-defined (see Harsanyi (1975)). For any specific vector $p_{\omega}$ of prior probability distributions, almost all games $\Gamma_{\omega}$ will give rise to a well-defined linear tracing procedure (see Chapter 3 ).

\subsection{Stochastic tracing procedure}

For the remainder of this chapter, let a stochastic game $\Gamma$ and a prior $p \in \Sigma$ be given. Because the prior $p$ is an element from $\Sigma$ and there exists a stationary best response to stationary strategies, each player $i$ has a stationary best response to $p^{-i}$.

For every $t \in[0,1]$, the stochastic tracing procedure generates a stationary equilibrium of the stochastic game $\Gamma^{t}=\left\langle N, \Omega,\left\{\sum_{\omega}^{i}\right\}_{\omega \in \Omega, i \in N},\left\{V^{i}(t)\right\}_{i \in N}\right\rangle$, where the total expected discounted payoff function $V^{i}(t)$ of player $i$ is defined such that

$$
V^{i}(0 ; \omega, \rho)=U^{i}\left(\omega, p^{-i}, \rho^{i}\right) \quad \text { and } \quad V^{i}(1 ; \omega, \rho)=U^{i}(\omega, \rho)
$$

The stochastic game $\Gamma^{0}$ corresponds to a trivial stochastic game, where all players believe that all their opponents play with probability 1 according to the prior belief. The stochastic game $\Gamma^{1}$ coincides with the original stochastic game $\Gamma$. Alternative definitions of $V^{i}(t)$ for $t \in(0,1)$ will give rise to alternative stochastic tracing procedures.

Given a proper definition of $V^{i}(t), i \in N$, for $t \in(0,1)$, the stochastic tracing procedure $\mathcal{S}(\Gamma, p)$ is defined as the set of pairs $(t, \rho)$ for which it holds that $\rho$ is a stationary equilibrium of the stochastic game $\Gamma^{t}$, i.e.

$$
\mathcal{S}(\Gamma, p)=\left\{(t, \rho) \in[0,1] \times \Sigma \mid \rho^{i} \text { is a best response to } \rho^{-i} \text { in } \Gamma^{t} \text { for all } i \in N\right\} .
$$

The stochastic tracing procedure is said to be feasible if there exists a path in $\mathcal{S}(\Gamma, p)$ connecting a best response against the prior to a stationary equilibrium of the stochastic game $\Gamma$, i.e. there exists a continuous function $\gamma:[0,1] \rightarrow \mathcal{S}(\Gamma, p)$ such that $\gamma(0) \in \mathcal{S}(\Gamma, p) \cap(\{0\} \times \Sigma)$ and $\gamma(1) \in \mathcal{S}(\Gamma, p) \cap(\{1\} \times \Sigma)$. In general there may be many trajectories $\gamma([0,1])$ that link a stationary equilibrium of $\Gamma^{0}$ to a stationary equilibrium of $\Gamma^{1}$. If this trajectory is unique, then 
$\mathcal{S}(\Gamma, p)$ is said to be well-defined. If the stochastic tracing procedure is well-defined, then it selects a unique stationary equilibrium of the stochastic game $\Gamma$.

The concepts stochastic tracing procedure, feasibility and well-definedness are all based on the definition of $V^{i}(t), i \in N$, for $t \in(0,1)$. There are at least four a priori reasonable ways in which $V^{i}(t)$ can be defined to extend the linear tracing procedure of Harsanyi and Selten to the setting of stochastic games. Choices have to be made whether a player holds correlated beliefs within a state or not, and whether a player holds correlated beliefs across time or not.

Correlation within states, $\mathrm{C}(\mathrm{S})$, means that when a player knows that some opponent plays according to the prior (which he expects with probability $1-t$ ), he expects all opponents to play according to the prior. Alternatively, absence of correlation within states, I(S), implies that even when a player knows that some opponent is playing according to the prior he may not infer that other opponents are playing according to the prior; all opponents are expected to play $(1-t) p^{i}+t \rho^{i}$, independent from one another.

Correlation across time, $\mathrm{C}(\mathrm{T})$, means that when a player knows that some opponent plays according to the prior today, he expects that opponent to play according to the prior in all future stages. Absence of correlation across time, I(T), implies that even when a player knows that some opponent is playing according to the prior today, this opponent might not play according to the prior in future stages. In all future events he faces independent lotteries which assign probability $1-t$ to play against the prior strategies of his opponents.

In the rationalizability literature the issue of "correlated beliefs" versus "uncorrelated beliefs" is often discussed. The second requires players to believe that the opponent players choose their strategies independently, while the first does not. Rationalizability with uncorrelated beliefs has been studied extensively by Bernheim (1984) and Pearce (1984). The correlated rationalizability concept is defined by Brandenburger and Dekel (1987) and is related to the correlated equilibrium concept of Aumann (1974, 1987).

To analyze the four possible extensions, the four alternatives have to be defined properly by specifying the corresponding functions $V^{i}(t)$. Therefore, this section contains four subsections, with each subsection devoted to one of the alternatives.

\subsubsection{Alternative 1: C(S), I(T)}

Suppose beliefs are correlated within states, but not across time. Because here correlation within states is assumed, each player expects that in stochastic game $\Gamma^{t}$ all opponents are playing according to the prior with probability $1-t$ and that all opponents are playing strategically with probability $t$. So, in the case of three or more players it is excluded that one opponent plays according to the prior whereas simultaneously another opponent plays strategically. The additional assumption of absence of correlation across time causes that each player faces this lottery 
at every stage. Therefore, the total discounted payoff to player $i$ in stochastic game $\Gamma^{t}$ when the initial state is $\omega$ and $\rho$ is played, equals

$$
\begin{aligned}
V_{\mathrm{C}(\mathrm{S}), \mathrm{I}(\mathrm{T})}^{i}(t ; \omega, \rho)= & (1-t) u^{i}\left(\omega, p_{\omega}^{-i}, \rho_{\omega}^{i}\right)+t u^{i}\left(\omega, \rho_{\omega}\right) \\
& +\delta \cdot \sum_{\bar{\omega} \in \Omega}\left[(1-t) \pi\left(\bar{\omega} \mid \omega, p_{\omega}^{-i}, \rho_{\omega}^{i}\right)+t \pi\left(\bar{\omega} \mid \omega, \rho_{\omega}\right)\right] V_{\mathrm{C}(\mathrm{S}), \mathrm{I}(\mathrm{T})}^{i}(t ; \bar{\omega}, \rho) .
\end{aligned}
$$

Stationarity implies that this payoff can be written by means of a recurrent relation. This stationarity results from the assumption that the beliefs of the players depend only on the state and not at the stage in which the state is reached.

\subsubsection{Alternative 2: C(S), C(T)}

When correlation as well within states as across time is assumed, again each player expects that in a stochastic game $\Gamma^{t}$ all opponents are playing according to the prior with probability $1-t$ and that all opponents are playing strategically with probability $t$. But now, unlike in the previous case, correlation across time is assumed. This means that when a player knows that some opponent plays according to the prior today, he expects that opponent to play according to the prior in all future stages. Apparently, unlike in the previous case each player faces this lottery only once and this will be at $k=0$. Therefore, the total discounted payoff to player $i$ in stochastic game $\Gamma^{t}$ when the initial state is $\omega$ and $\rho$ is played, is

$$
V_{\mathrm{C}(\mathrm{S}), \mathrm{C}(\mathrm{T})}^{i}(t ; \omega, \rho)=(1-t) U^{i}\left(\omega, p^{-i}, \rho^{i}\right)+t U^{i}(\omega, \rho) .
$$

A solution $(t, \rho)$ for $t \in(0,1)$ in $\mathcal{S}(\Gamma, p)$ is not necessarily a stationary equilibrium of the game $\Gamma^{t}$. A player $i$ might have a non-stationary strategy that is better than $\rho^{i}$ against $\rho^{-i}$ in $\Gamma^{t}$. Namely, at $k=0$ all players make their stationary decisions believing that with probability $1-t$ all opponents play according to the prior and with probability $t$ they play strategically. In fact, in $k=0$ all players are facing a lottery. Once the players are in $k=1$, they know the outcome of the lottery, i.e. in $k=1$ they have information on the strategies played in $k=0$ ( $p^{-i}$ or $\rho^{-i}$ ). It might be better, and most of the time it will be better, to revise their strategy having this knowledge, which would lead to a non-stationary strategy.

\subsubsection{Alternative 3: I(S), I(T)}

In this subsection the situation in which absence of correlation both within states and across time is assumed is considered. Absence of correlation within states means that in the stochastic game $\Gamma^{t}$ a player expects that each opponent is playing independently according to the prior with probability $1-t$ and playing strategically with probability $t$. The additional assumption of absence of correlation across time causes every player to face this lottery at every stage. Clearly, 
these assumptions imply the total discounted payoff to player $i$ in stochastic game $\Gamma^{t}$ when the initial state is $\omega$ and the strategy $\rho$ is played to be equal to

$$
V_{\mathrm{I}(\mathrm{S}) \mathrm{I}(\mathrm{T})}^{i}(t ; \omega, \rho)=U^{i}\left(\omega,(1-t) p^{-i}+t \rho^{-i}, \rho^{i}\right) .
$$

Just like in Subsection 5.4.1 it is possible, because of absence of correlation across time, to rewrite the total discounted payoffs in recurrent form:

$$
\begin{aligned}
V_{\mathrm{I}(\mathrm{S}), \mathrm{I}(\mathrm{T})}^{i}(t ; \omega, \rho)= & u^{i}\left(\omega,(1-t) p_{\omega}^{-i}+t \rho_{\omega}^{-i}, \rho_{\omega}^{i}\right) \\
& +\delta \cdot \sum_{\bar{\omega} \in \Omega} \pi\left(\bar{\omega} \mid \omega,(1-t) p_{\omega}^{-i}+t \rho_{\omega}^{-i}, \rho_{\omega}^{i}\right) V_{\mathrm{I}(\mathrm{S}), \mathrm{I}(\mathrm{T})}^{i}(t ; \bar{\omega}, \rho) .
\end{aligned}
$$

Since the decision problem a player faces depends on the state only, his problem is stationary no matter at which stage of the game the state is realized. Assuming that beliefs are not correlated across time captures, therefore, the presence of stationarity. Stationarity means that a recurrent relation can be given. A direct result is that best stationary responses are best responses in the broader class of behavior strategies as well.

\subsubsection{Alternative 4: I(S), C(T)}

When absence of correlation within states, but correlation across time is assumed, again each player expects that in stochastic game $\Gamma^{t}$ each opponent is playing according to the prior with probability $1-t$ and playing strategically with probability $t$. But this time, in contrast to Subsection 5.4.3, correlation across time is assumed. This means that when a player knows that some opponent plays according to the prior today, he expects that opponent to play according to the prior in all future stages. Therefore, the total discounted payoff to player $i$ in stochastic game $\Gamma^{t}$ when the initial state is $\omega$ and $\rho$ is played, equals

$$
V_{\mathrm{I}(\mathrm{S}), \mathrm{C}(\mathrm{T})}^{i}(t ; \omega, \rho)=\sum_{S \subseteq N \backslash\{i\}}(1-t)^{|S|} t^{|N \backslash S|-1} U^{i}\left(\omega, p^{S}, \rho^{N \backslash S}\right) .
$$

In the formula, $(1-t)^{|S|} t^{|N \backslash S|-1}$ is the probability that the opponents from $S \subseteq N \backslash\{i\}$ are playing according to the prior, whereas the other opponents are playing strategically. When player $i$ 's opponents from $S$ are playing according to $p$ and the others according to $\rho$, his total discounted payoff is $U^{i}\left(\omega, p^{S}, \rho^{N \backslash S}\right)$. Player $i$ 's payoff in the game $\Gamma^{t}$ is therefore the sum over all subsets $S$ of $N \backslash\{i\}$ of the probability that only the players from $S$ play according to $p$ (given $t$ ) times the total expected discounted payoff in that case.

Like in Subsection 5.4.2, a solution $(t, \rho)$ for $t \in(0,1)$ in $\mathcal{S}(\Gamma, p)$ is not necessarily a stationary equilibrium of the game $\Gamma^{t}$. A player $i$ might have a non-stationary strategy that performs better than $\rho^{i}$ against $\rho^{-i}$ in $\Gamma^{t}$. Apparently, a best stationary response is not necessarily a best response in the broader class of behavior strategies. 


\subsubsection{Properties}

In order to be useful as a selection theory, it is important that for almost all stochastic games the stochastic tracing procedure determines a unique stationary equilibrium. When well-definedness holds, one can be sure of selecting a unique stationary equilibrium. The following result is shown in the next chapter, see the proof of Theorem 6.6.

Theorem 5.1 Assume $C(S)$ and $I(T)$. There exists an open set of stochastic games and priors $(\Gamma, p)$ with full Lebesgue measure such that the stochastic tracing procedure is well-defined.

Repeating and modifying the proof of the former theorem it is possible to prove the following result.

Theorem 5.2 Assume $I(S)$ and $I(T)$. There exists an open set of stochastic games and priors $(\Gamma, p)$ with full Lebesgue measure such that the stochastic tracing procedure is well-defined.

For the alternatives where $\mathrm{C}(\mathrm{T})$ is assumed, it is not possible to rewrite the payoffs into a recurrent relation. Therefore, to prove similar theorems with $\mathrm{C}(\mathrm{T})$ assumed, calls for different techniques of proof. Nevertheless, it should be expected that generic well-definedness holds as well when C(T) is assumed. Since the number of variables is one less than the number of constraints imposed by the concept of stationary equilibrium, there is one degree of freedom left. Under suitable transversality conditions, this should be sufficient to show well-definedness.

\subsection{Analysis of alternatives}

The analysis proceeds by analyzing the four alternatives defined so far. The first part of this section is devoted to lay bare that the choice about beliefs within states matter. This is done by a well-chosen example. In the second part of this section the same is done for the assumption with respect to beliefs across time.

\subsubsection{Beliefs within states}

In this subsection an example is created for which it matters whether correlation or absence of correlation within states is assumed, in the sense that different stationary equilibria will be selected starting from the same prior. Before doing so, it is important to know the minimal size of such an example. In case there are two players, assuming absence or presence of correlation within states does not matter since the number of opponents is one.

Proposition 5.3 For $n=2$, the stochastic tracing procedure based on $I(S)$ is identical to the one based on $C(S)$. 
So, in order to construct an example with the desired properties the number of players must be larger than or equal to 3 .

Consider the normal form game $\Gamma$ with three players each having two strategies of Figure 5.1. This is a special case of a stochastic game, namely one with only one state and discount factor $\delta$ equal to 0 . The left element in the first upper-left box is the payoff to player 1 if all players play their first strategy. The advantage of considering a normal form game, is that it disconnects

\begin{tabular}{|c|c|c|c|c|}
\hline \multicolumn{3}{|c|}{$s^{2}$} & \multirow[b]{2}{*}{$0,0,0$} & \multirow[b]{2}{*}{$0,0,0$} \\
\hline$s^{1}$ & $2,2,2$ & $0,0,0$ & & \\
\hline$s^{1^{\prime}}$ & $0,0,0$ & $0,0,0$ & $0,0,0$ & $1,1,1$ \\
\hline
\end{tabular}

Figure 5.1: Normal form game $\Gamma$.

assumptions on beliefs within states and assumptions on beliefs across time. Note that this game possesses three stationary equilibria: the pure stationary equilibria $\left(s^{1} ; s^{2} ; s^{3}\right)$ and $\left(s^{1^{\prime}} ; s^{2^{\prime}} ; s^{3^{\prime}}\right)$, and a mixed stationary equilibrium at which all players play their first strategy with probability $\sqrt{2}-1$ and their second with probability $2-\sqrt{2}$. Because the discount factor is taken equal to 0 , it does not matter whether $\mathrm{I}(\mathrm{T})$ or $\mathrm{C}(\mathrm{T})$ is assumed.

Take the following prior:

$$
p=\left(\left(\frac{1}{6}, \frac{5}{6}\right) ;\left(\frac{1}{2}, \frac{1}{2}\right) ;\left(\frac{2}{3}, \frac{1}{3}\right)\right) .
$$

Note that for the best response against the prior it does not matter whether $\mathrm{C}(\mathrm{S})$ or $\mathrm{I}(\mathrm{S})$ is assumed. Both alternatives lead to the same best response to all possible priors.

Here, the prior is chosen such that the starting point is $\left(0,\left(s^{1} ; s^{2^{\prime}} ; s^{3^{\prime}}\right)\right)$. Player 1 starts playing the equilibrium strategy of the first pure stationary equilibrium and players 2 and 3 start playing the equilibrium strategy of the second pure stationary equilibrium. To obtain a stationary equilibrium, at least one player has to switch to another pure strategy. It turns out that in the case of $\mathrm{I}(\mathrm{S})$, player 2 or 3 is switching his strategy before player 1, whereas in the other case, $\mathrm{C}(\mathrm{S})$, player 1 is the first player willing to switch his strategy.

\section{When $\mathbf{I}(\mathbf{S})$ is assumed}

When absence of correlation within states is assumed, $\Gamma^{t}$ has the form as depicted in Figure 5.2. The upper-left element of the most upper-left box, $\frac{1}{3}(1+t)(2+t)$, is the payoff to player 1 if he plays $s^{1}$ and he expects player 2 to play according to the prior with probability $1-t$ and $s^{2}$ 

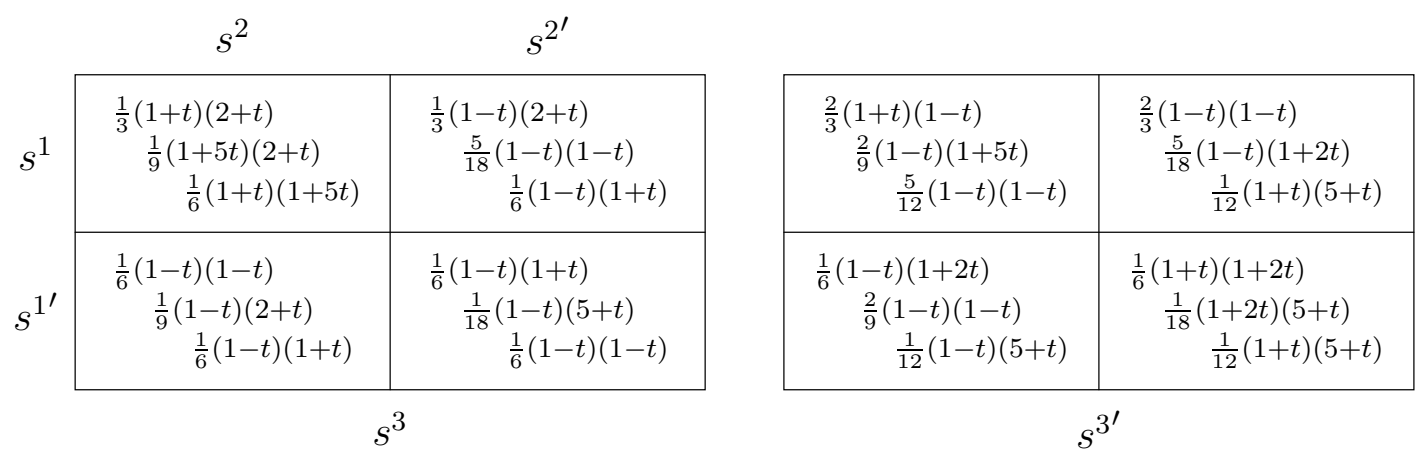

Figure 5.2: Game $\Gamma^{t}$ when $\mathrm{I}(\mathrm{S})$ is assumed.

with probability $t$ and expects player 3 to play according to the prior with probability $1-t$ and $s^{3}$ with probability $t$. So, player 1 expects player 2 to play $\left(\frac{1}{2}(1-t)+t, \frac{1}{2}(1-t)\right)$ and player 3 to play $\left(\frac{2}{3}(1-t)+t, \frac{1}{3}(1-t)\right)$ and the expected payoff when playing $s^{1}$ is therefore equal to $\left(\frac{1}{2}(1-t)+t\right)\left(\frac{2}{3}(1-t)+t\right) 2=\frac{1}{3}(1+t)(2+t)$, and so on for the other entries.

For all $t \in[0,1]$ it is possible to compute all stationary equilibria of $\Gamma^{t}$. Since the only matter of interest is the stationary equilibrium selected, only the path starting at $t=0$ and terminating in $t=1$ is determined. Obviously, by the way the prior is constructed, the path starts in the point $\left(s^{1} ; s^{2^{\prime}} ; s^{3^{\prime}}\right)$. This point is a stationary equilibrium of $\Gamma^{t}$ as long as $t \leq 1 / 10$. When $t=1 / 10$, player 2 is indifferent between his two pure strategies. So, at $t=1 / 10$ the path jumps from $\left(s^{1} ; s^{2^{\prime}} ; s^{3^{\prime}}\right)$ to $\left(s^{1} ; s^{2} ; s^{3^{\prime}}\right)$. Next, the point $\left(s^{1} ; s^{2} ; s^{3^{\prime}}\right)$ is a stationary equilibrium of $\Gamma^{t}$ until $t=1 / 5(29-2 \sqrt{189}) \approx 0.3009$, when player 3 is indifferent between his two pure strategies. Here, in $t=1 / 5(29-2 \sqrt{189})$, the path jumps from $\left(s^{1} ; s^{2} ; s^{3^{\prime}}\right)$ to $\left(s^{1} ; s^{2} ; s^{3}\right)$ which is a stationary equilibrium of $\Gamma^{t}$ for all $t \in[1 / 5(29-2 \sqrt{189}), 1]$. The point $\left(s^{1} ; s^{2} ; s^{3}\right)$ is therefore the stationary equilibrium selected by the stochastic tracing procedure when $\mathrm{I}(\mathrm{S})$ is assumed. The path is displayed graphically in Figure 5.3.

This figure shows a cube moving from $t=0$ to $t=1$. The cube itself displays the set of stationary strategy-combinations. The strategic possibilities of player 1, player 2 and player 3 are displayed in the horizontal, diagonal, respectively vertical direction. The point marked with a circle (o) is the point where all players play their first pure strategy. The two bullets $(\bullet)$ which are not endpoints of the feasible path of the stochastic tracing procedure display the remaining two equilibria. In the figure it looks like if the feasible path moves backwards in the homotopyparameter $t$. This is actually not the case as the path moves along the edge of the cube in $t=0.1$.

\section{When $\mathrm{C}(\mathrm{S})$ is assumed}

When correlation within states is assumed, $\Gamma^{t}$ has the form as depicted in Figure 5.4. The first element in the first box is the payoff to player 1 if he plays $s^{1}$ and he expects players 2 and 3 


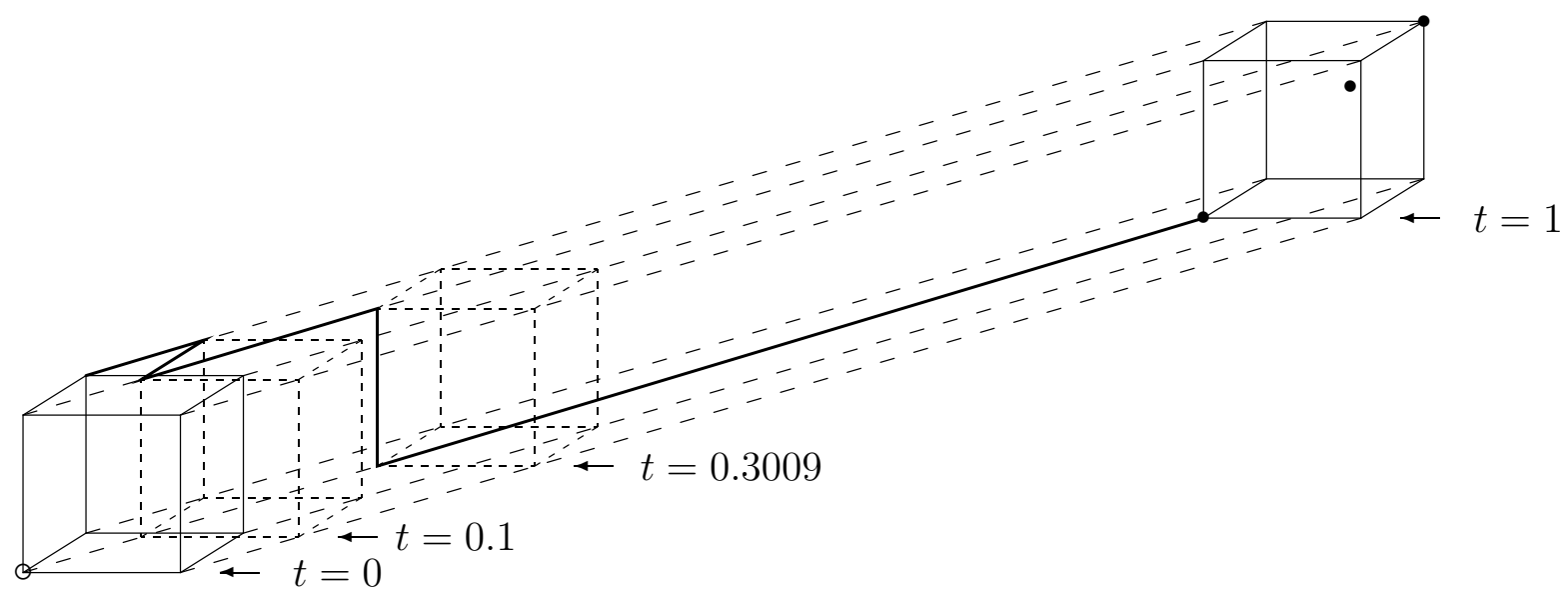

Figure 5.3: Feasible path when $\mathrm{I}(\mathrm{S})$ is assumed.

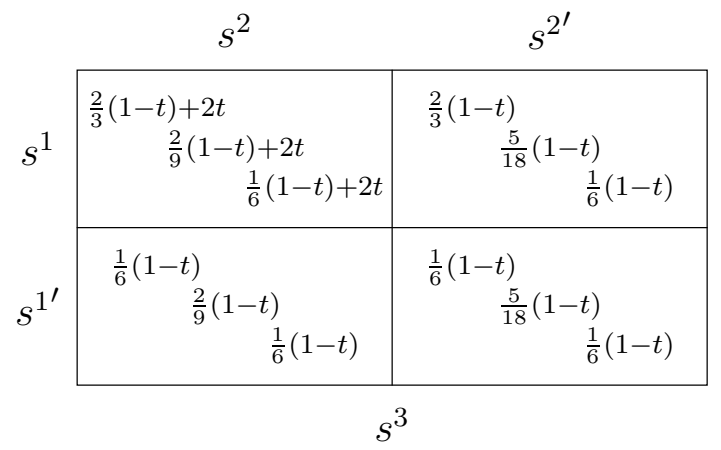

\begin{tabular}{|c|c|}
\hline $\begin{array}{l}\frac{2}{3}(1-t) \\
\quad \frac{2}{9}(1-t) \\
\quad \frac{5}{12}(1-t)\end{array}$ & $\begin{array}{l}\frac{2}{3}(1-t) \\
\quad \frac{5}{18}(1-t) \\
\quad \frac{5}{12}(1-t)\end{array}$ \\
\hline $\begin{array}{l}\frac{1}{6}(1-t) \\
\quad \frac{2}{9}(1-t) \\
\quad \frac{5}{12}(1-t)\end{array}$ & $\begin{array}{l}\frac{1}{6}(1-t)+t \\
\quad \frac{5}{18}(1-t)+t \\
\quad \frac{5}{12}(1-t)+t\end{array}$ \\
\hline
\end{tabular}

Figure 5.4: Game $\Gamma^{t}$ when $\mathrm{C}(\mathrm{S})$ is assumed.

to play according to the prior with probability $1-t$ and their first pure strategy with probability $t$. So, player 1 expects with probability $1-t$ that players 2 and 3 are playing $\left(\frac{1}{2}, \frac{1}{2}\right)$ and $\left(\frac{2}{3}, \frac{1}{3}\right)$, respectively, and with probability $t$ that players 2 and 3 are playing $s^{2}$ and $s^{3}$, respectively. The expected payoff to player 1 when playing $s^{1}$ will therefore be equal to $\left((1-t) \frac{1}{2} \frac{2}{3}+t\right) 2=$ $\frac{2}{3}(1-t)+2 t$.

Again, it is possible to compute all stationary equilibria of $\Gamma^{t}$, for $t \in[0,1]$. And, again, restriction to determine the path starting at $t=0$ and terminating in $t=1$ is made. Just as before, the path starts in the point $\left(s^{1} ; s^{2^{\prime}} ; s^{3^{\prime}}\right)$. This point is a stationary equilibrium point as long as $t \leq 1 / 3$. When $t=1 / 3$, player 1 is indifferent between both his pure strategies and will be the first player to switch to another pure strategy unlike in the previous case. At $t=1 / 3$, the path jumps from $\left(s^{1} ; s^{2^{\prime}} ; s^{3^{\prime}}\right)$ to $\left(s^{1^{\prime}} ; s^{2^{\prime}} ; s^{3^{\prime}}\right)$. The point $\left(s^{1^{\prime}} ; s^{2^{\prime}} ; s^{3^{\prime}}\right)$ is a stationary equilibrium of $\Gamma^{t}$ up to $t=1$ and this point is therefore the stationary equilibrium selected by the stochastic tracing procedure when $\mathrm{C}(\mathrm{S})$ is assumed. The path is displayed graphically in Figure 5.5. 


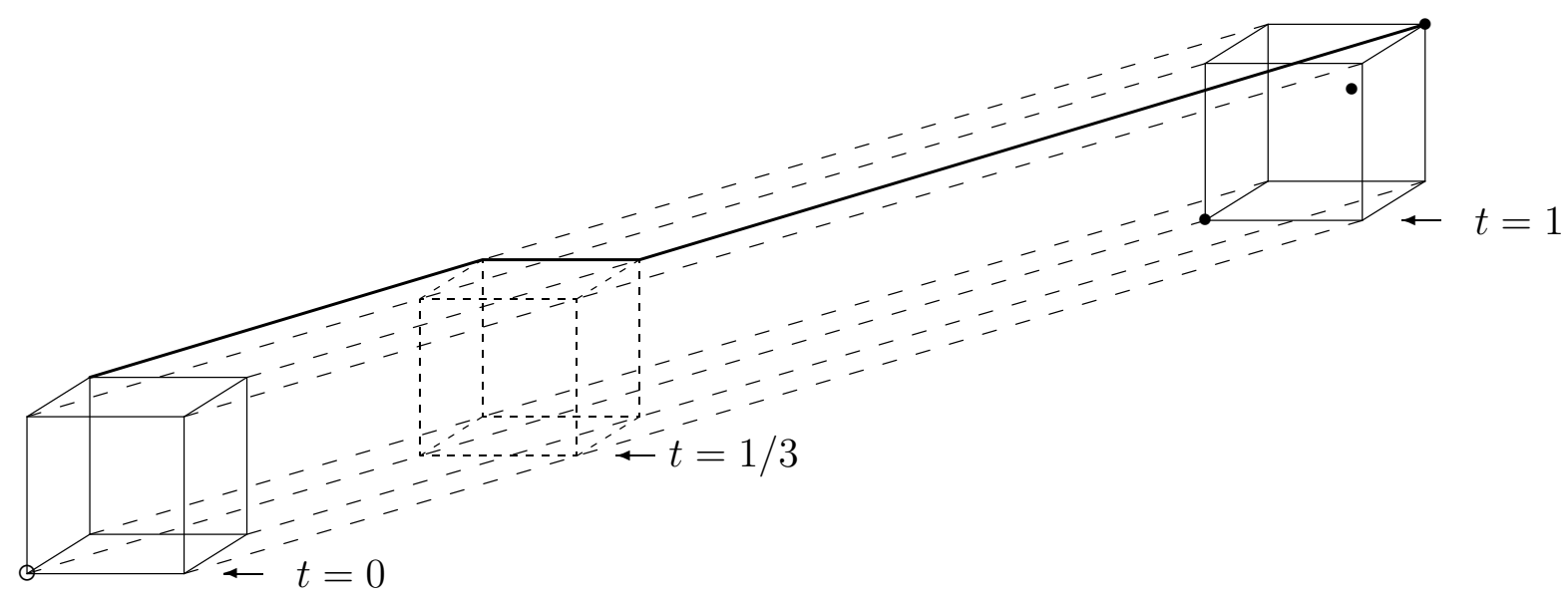

Figure 5.5: Feasible path when $\mathrm{C}(\mathrm{S})$ is assumed.

\subsubsection{Beliefs across time}

Again, it is important to know the minimal size of an example for which it matters whether correlation or absence of correlation across time is assumed. In the next proposition it is claimed that $\mathrm{C}(\mathrm{T})$ and $\mathrm{I}(\mathrm{T})$ select the same stationary equilibrium for stochastic games with one state (repeated game), when the process never leaves a state (finite number of independent repeated games), or when future payoffs are ignored (finite number of normal games).

Proposition 5.4 When $\pi=\mathbb{1}_{\Omega}$ or $\delta=0$, the stochastic tracing procedure based on $I(T)$ is identical to the one based on $C(T)$.

Proof Consider a repeated game, i.e. $|\Omega|=1$ and therefore trivially $\pi=\mathbb{1}_{\Omega}$. In this case, the total discounted payoffs for the four alternatives are:

$$
\begin{aligned}
& V_{\mathrm{C}(\mathrm{S}), \mathrm{I}(\mathrm{T})}^{i}(t ; \rho)=(1-t) u^{i}\left(p^{-i}, \rho^{i}\right)+t u^{i}(\rho)+\delta V_{\mathrm{C}(\mathrm{S}), \mathrm{I}(\mathrm{T})}^{i}(t ; \rho), \\
& V_{\mathrm{C}(\mathrm{S}), \mathrm{C}(\mathrm{T})}^{i}(t ; \rho)=(1-t) U^{i}\left(p^{-i}, \rho^{i}\right)+t U^{i}(\rho), \\
& V_{\mathrm{I}(\mathrm{S}), \mathrm{I}(\mathrm{T})}^{i}(t ; \rho)=u^{i}\left((1-t) p^{-i}+t \rho^{-i}, \rho^{i}\right)+\delta V_{\mathrm{I}(\mathrm{S}), \mathrm{I}(\mathrm{T})}^{i}(t ; \rho),
\end{aligned}
$$

and

$$
V_{\mathrm{I}(\mathrm{S}), \mathrm{C}(\mathrm{T})}^{i}(t ; \rho)=\sum_{S \subseteq N \backslash\{i\}}(1-t)^{|S|} t^{|N \backslash S|-1} U^{i}\left(p^{S}, \rho^{N \backslash S}\right) .
$$

First, note that

$$
\begin{aligned}
V_{\mathrm{C}(\mathrm{S}), \mathrm{C}(\mathrm{T})}^{i}(t ; \rho) & =(1-t) U^{i}\left(p^{-i}, \rho^{i}\right)+t U^{i}(\rho) \\
& =(1-t)\left[u^{i}\left(p^{-i}, \rho^{i}\right)+\delta U^{i}\left(p^{-i}, \rho^{i}\right)\right]+t\left[u^{i}(\rho)+\delta U^{i}\left(\rho^{i}\right)\right] \\
& =(1-t) u^{i}\left(p^{-i}, \rho^{i}\right)+t u^{i}(\rho)+\delta \cdot\left[(1-t) U^{i}\left(p^{-i}, \rho^{i}\right)+t U^{i}(\rho)\right] \\
& =(1-t) u^{i}\left(p^{-i}, \rho^{i}\right)+t u^{i}(\rho)+\delta V_{\mathrm{C}(\mathrm{S}), \mathrm{C}(\mathrm{T})}^{i}(t ; \rho) .
\end{aligned}
$$


And secondly, note that

$$
\begin{aligned}
V_{\mathrm{I}(\mathrm{S}), \mathrm{C}(\mathrm{T})}^{i}(t ; \rho)= & \sum_{S \subseteq N \backslash\{i\}}(1-t)^{|S|} t^{|N \backslash S|-1} U^{i}\left(p^{S}, \rho^{N \backslash S}\right) \\
= & \sum_{S \subseteq N \backslash\{i\}}(1-t)^{|S|} t^{|N \backslash S|-1}\left[u^{i}\left(p^{S}, \rho^{N \backslash S}\right)+\delta U^{i}\left(p^{S}, \rho^{N \backslash S}\right)\right] \\
= & \sum_{S \subseteq N \backslash\{i\}}(1-t)^{|S|} t^{|N \backslash S|-1} u^{i}\left(p^{S}, \rho^{N \backslash S}\right) \\
& \quad+\delta \cdot \sum_{S \subseteq N \backslash\{i\}}(1-t)^{|S|} t^{|N \backslash S|-1} U^{i}\left(p^{S}, \rho^{N \backslash S}\right) \\
= & u^{i}\left((1-t) p^{-i}+t \rho^{-i}, \rho^{i}\right)+\delta V_{\mathrm{I}(\mathrm{S}), \mathrm{C}(\mathrm{T})}^{i}(t ; \rho) .
\end{aligned}
$$

Finally, observe that

$$
V_{\mathrm{C}(\mathrm{S}), \mathrm{I}(\mathrm{T})}^{i}(t ; \rho)=V_{\mathrm{C}(\mathrm{S}), \mathrm{C}(\mathrm{T})}^{i}(t ; \rho),
$$

and that

$$
V_{\mathrm{I}(\mathrm{S}), \mathrm{I}(\mathrm{T})}^{i}(t ; \rho)=V_{\mathrm{I}(\mathrm{S}), \mathrm{C}(\mathrm{T})}^{i}(t ; \rho)
$$

for all $(t ; \rho) \in[0,1] \times \Sigma$. So, for repeated games $\mathrm{C}(\mathrm{T})$ and $\mathrm{I}(\mathrm{T})$ are equivalent.

From this, the equivalence between $\mathrm{C}(\mathrm{T})$ and $\mathrm{I}(\mathrm{T})$ follows trivially when $\pi=1_{\Omega}$ as being a finite number of independent repeated games.

When $\delta=0$, the stochastic game is equivalent to a one shot game in which time plays no role when stationary strategies are assumed.

Consider the stochastic game $\Gamma$ of Figure 5.6. The stochastic game displayed in that figure is a
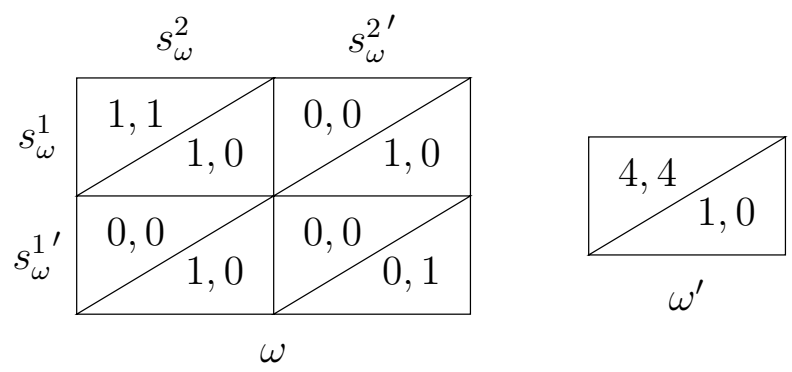

$$
\delta=1 / 3
$$

Figure 5.6: Stochastic game $\Gamma$.

stochastic game with two players, two states and discount factor $\delta$ equal to $1 / 3$. In the first state both players have two pure strategies, whereas in the second state both players have one strategy. The upper-left corner of each box contains the payoffs. The transition probabilities are displayed in the lower-right corner, where the first element is the probability that in the next stage the game will be in the first state, the second element is the probability that the game will be in the second state at the next stage. 
When both players play their second strategy in state $\omega$, they both expect to earn 0 at the current stage, go to state $\omega^{\prime}$, earn 4 at the next stage and then return to state $\omega$. Notice that the game at $\omega^{\prime}$ is completely degenerate. There is no need for players to make a choice. They simply collect a payoff of 4 . When $\omega$ is the initial state, both players expect to earn $\frac{4}{3}\left(1+\frac{1}{9}+\frac{1}{81}+\ldots\right)=\frac{3}{2}$ in total when both always play their second pure strategy. Suppose the box that belongs to strategy-combination $\left(s_{\omega}^{1} ; s_{\omega}^{2}\right)$ had 1,1 in the upper-left corner and 1,0 in the lower-right corner. Then the game is always in state $\omega$ when $\omega$ is the initial state, so coincides with a repeated game. When again both players play their second pure strategy at all times, they both expect again to earn $1+\frac{1}{3}+\frac{1}{9}+\ldots=\frac{3}{2}$. Nevertheless, the two resulting stochastic games are not strategically equivalent when mixed strategies are played. It turns out that $\left(\left(\frac{1}{2}, \frac{1}{2}\right) ;\left(\frac{1}{2}, \frac{1}{2}\right)\right)$ is not a stationary equilibrium in the stochastic game of Figure 5.6 unlike in the adapted stochastic game (the repeated game). Although the stochastic game of Figure 5.6 is close to a repeated game, for which $\mathrm{C}(\mathrm{T})$ and $\mathrm{I}(\mathrm{T})$ are equivalent, it is certainly not.

The stochastic game of Figure 5.6 possesses three stationary equilibria: the pure stationary equilibria $\left(s_{\omega}^{1} ; s_{\omega}^{2}\right)$ and $\left(s_{\omega}^{1} ; s_{\omega}^{2}\right)$, and the symmetric mixed stationary equilibrium where both players play their first strategy with probability $4-2 \sqrt{3} \approx 0.5359$ and the other strategy with the rest of the probability mass.

Just like in the previous example, the prior is chosen such that the starting point of the stochastic tracing procedure is not a stationary equilibrium strategy-combination of the original game. This can be arranged by taking for one player the prior play of the first strategy smaller than $4-2 \sqrt{3}$ and the reverse for the other. Without loss of generality, let the first player mentioned be player 1 . Given such a prior, player 1 will prefer strategy $s_{\omega}^{1}$ and player 2 will prefer strategy $s_{\omega}^{2}$. Consider the prior

$$
p=\left(\left(\frac{1}{2}, \frac{1}{2}\right) ;\left(\frac{2}{3}, \frac{1}{3}\right)\right)
$$

which satisfies this property. Note that the starting point, in this case $\left(s_{\omega}^{1} ; s_{\omega}^{2}\right)$, is independent of the choice made between the two assumptions $\mathrm{I}(\mathrm{T})$ and $\mathrm{C}(\mathrm{T})$. Because the stochastic game depicted is a game with two players, it does not matter whether $C(S)$ or $I(S)$ is assumed.

\section{When $I(T)$ is assumed}

In Figure 5.7 the stage game for $t=0$ is given. The figure is almost similar to Figure 5.6. The difference is found in the lower-right corner of the boxes, which is divided into two sectors. The lower-left sector displays the transition probabilities as player 1 thinks they are, the upper-right sector the transition probabilities as player 2 thinks they are. For example, when player 1 plays $s_{\omega}^{1 \prime}$ he expects to receive 0 and expects that in the next stage the state will be $\omega$ with probability $2 / 3$ and $\omega^{\prime}$ with probability $1 / 3$. 

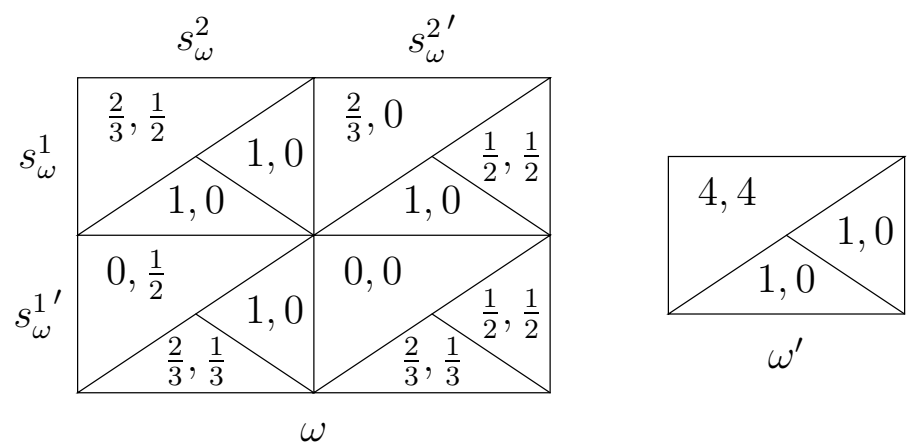

$\delta=1 / 3$

Figure 5.7: Game $\Gamma^{0}$ when $\mathrm{I}(\mathrm{T})$ is assumed.

The stage games $\Gamma_{\omega}^{t}$ can now be defined as $\Gamma_{\omega}^{t}=(1-t) \Gamma_{\omega}^{0}+t \Gamma_{\omega}^{1}$ for all $t \in(0,1)$. For all $t \in[0,1]$, it is possible to compute all stationary equilibria of $\Gamma^{t}$, but since only the stationary equilibrium selected is of interest, only the path starting at $t=0$ and terminating in $t=1$ is determined. As mentioned before, the path starts in the point $\left(s_{\omega}^{1} ; s_{\omega}^{2}{ }^{\prime}\right)$. This point is a stationary equilibrium point as long as $t \leq \frac{1}{2}(23-\sqrt{513}) \approx 0.1752$, the moment player 1 is indifferent between both his pure strategies. Player 2 is not willing to switch his strategy as long as $t \leq$ $\frac{1}{6}(11-\sqrt{97}) \approx 0.1919$. So, player 1 is the first to change his strategy and at $t=\frac{1}{2}(23-\sqrt{513})$ the path jumps from $\left(s_{\omega}^{1} ; s_{\omega}^{2 \prime}\right)$ to $\left(s_{\omega}^{1^{\prime}} ; s_{\omega}^{2 \prime}\right)$. This point is a stationary equilibrium point for all $t \in\left[\frac{1}{2}(23-\sqrt{513}), 1\right]$. The point $\left(s_{\omega}^{1 \prime} ; s_{\omega}^{2 \prime}\right)$ is therefore the stationary equilibrium selected by the stochastic tracing procedure when $\mathrm{I}(\mathrm{T})$ is assumed. The path is displayed graphically in Figure 5.8.

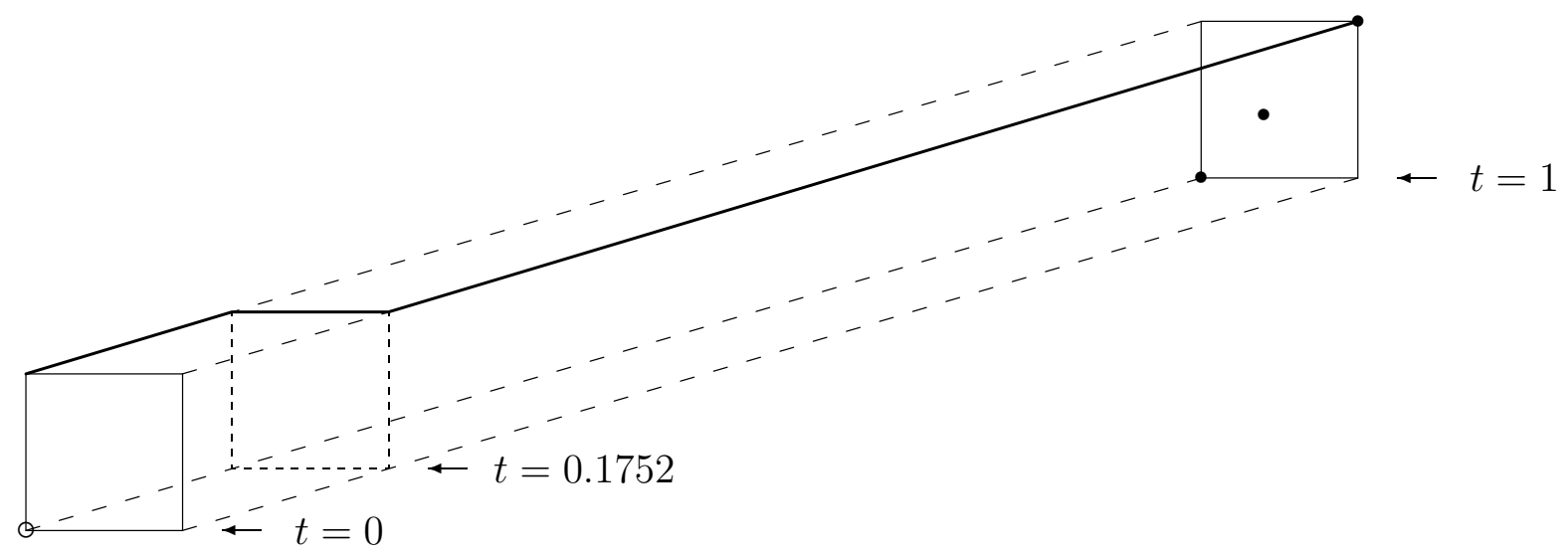

Figure 5.8: Feasible path when $\mathrm{I}(\mathrm{T})$ is assumed.

This figure shows the Cartesian product of the strategy space (a square) and the interval [0,1]. The strategic possibilities of player 1 and player 2 are displayed in the horizontal and vertical 
direction, respectively. The point marked with a circle corresponds to both players playing their first pure strategy.

\section{When $C(T)$ is assumed}

When $\mathrm{C}(\mathrm{T})$, correlation across time, is assumed, $\Gamma^{t}$ cannot be represented easily in a figure. Again, the path starts in the point $\left(s_{\omega}^{1} ; s_{\omega}^{2 \prime}\right)$. Given that player 2 plays $s_{\omega}^{2 \prime}$ during the whole play with probability $t$ and according to the prior during the whole play with probability $1-t$, the best stationary response of player 1 is playing $s_{\omega}^{1}$ as long as $t$ is less than $4 / 19$. When $t$ is larger than $4 / 19$ his other pure stationary strategy, that is $s_{\omega}^{1}$, will be his best stationary response. Vice versa, when player 2 expects player 1 to play according to the prior the whole stochastic game with probability $1-t$ and $s_{\omega}^{1}$ the whole play with probability $t$, his best stationary response is playing strategy $s_{\omega}^{2 \prime}$ when $t \leq 1 / 15$ and $s_{\omega}^{2}$ otherwise. So, player 2 will be the first player to switch strategy, since $1 / 15<4 / 19$. Therefore, at $t=1 / 15$, the path will jump from $\left(s_{\omega}^{1} ; s_{\omega}^{2}{ }^{\prime}\right)$ to $\left(s_{\omega}^{1} ; s_{\omega}^{2}\right)$. From $t=1 / 15$ up to $t=1$ the point $\left(s_{\omega}^{1} ; s_{\omega}^{2}\right)$ is the stationary equilibrium and is also the stationary equilibrium selected by the stochastic tracing procedure when $\mathrm{C}(\mathrm{T})$ is assumed. The path is plotted in Figure 5.9 and is displayed in the same style as Figure 5.8.

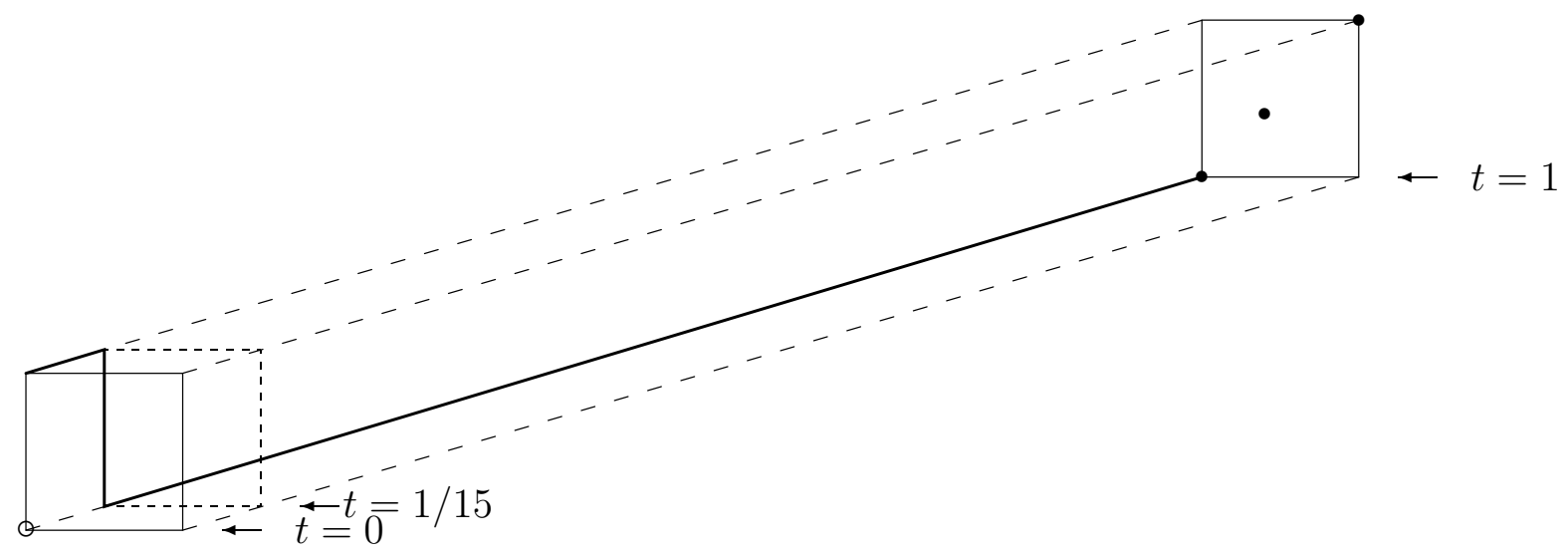

Figure 5.9: Feasible path when $\mathrm{C}(\mathrm{T})$ is assumed.

\subsubsection{Extending the linear tracing procedure}

The first subsection showed that the choice between assuming absence of correlation or assuming presence of correlation within states can cause different stationary equilibria to be selected. Although this was shown by means of a normal form game, the same result would have been found if it was made a repeated game by taking a positive discount factor, i.e. $\delta \in(0,1)$.

In the selection theory of Harsanyi and Selten (1988), the linear tracing procedure of Harsanyi (1975) is used to select a Nash equilibrium of a normal form game. The structure of the linear 
tracing procedure there assumed beliefs to be correlated. The most natural extension of the linear tracing procedure is therefore the one that assumes correlation within states. For instance, this choice implies that for repeated games, the stationary equilibrium which is selected is the repeated Nash equilibrium that is selected by the linear tracing procedure for the stage game using the same prior.

The second subsection showed that the choice between assuming absence of correlation or assuming presence of correlation across time can cause different stationary equilibria to be selected. When $\mathrm{C}(\mathrm{T})$ is assumed, a point $(t, \rho)$ on the feasible path is not necessarily a stationary equilibrium of $\Gamma^{t}$. For some player $i$ there might be a better behavior strategy than $\rho^{i}$ against $\rho^{-i}$ in $\Gamma^{t}$ which is non-stationary, i.e. a best stationary response is not necessarily a best response in the broader class of behavior strategies. However, assuming that beliefs are not correlated across time, that is assuming I(T), captures the assumption of stationarity. The beliefs of a player will depend only on the state reached and not on the stage at which it is reached. Therefore the assumption of $\mathrm{C}(\mathrm{S})$ and $\mathrm{I}(\mathrm{T})$ leads to a stochastic tracing procedure that is the most natural extension of the linear tracing procedure of Harsanyi and Selten to the environment of stochastic games.

\subsection{Summary}

In this chapter the linear tracing procedure of Harsanyi (1975) is extended in order to select on an equilibrium for stochastic games, more precisely, to select on a stationary equilibrium for stochastic games. There are four reasonable extensions. After having defined all four properly, it is shown by examples that these extensions are independent. The most natural extension assumes that players hold correlated beliefs within states, but do not hold correlated beliefs across time, since this is the only possible extension consistent with the formulation of Harsanyi for normal form games that captures stationarity. This extension is defined as the stochastic tracing procedure. In the next chapter it is proved that the stochastic tracing procedure is well-defined for almost all stochastic games. 


\section{Chapter 6}

\section{Computation of Stationary Equilibria}

This chapter is based on Herings and Peeters (2000) which is the first paper to introduce an algorithm to compute stationary equilibria in stochastic games, and shows convergence of the algorithm for almost all such games. Moreover, since in general the number of stationary equilibria is overwhelming, attention is paid to the issue of equilibrium selection. The stationary equilibrium computed is the one selected by the stochastic tracing procedure, an extension of the linear tracing procedure to the class of stochastic games that has been defined in the previous chapter. The algorithm belongs to the class of homotopy methods and is easily and robustly implemented on a computer using existing software routines to follow paths numerically. As a by-product, a recent result on the generic finiteness of stationary equilibria in stochastic games is extended to oddness of equilibria.

\subsection{Introduction}

Many economic situations of interest can be modeled as a stochastic game. Recent work of for instance Olley and Pakes (1996), Pakes and Ericson (1998), Pakes and McGuire (1996), and Bergemann and Välimäki (1996) is devoted to the application of stochastic games to problems emerging in the industrial organization literature. Another application within this stream of literature is presented in Chapter 7. Further progress in this research program can be made by developing methods to solve stochastic games numerically. Numerical solution methods allow researchers to go back and forth between the implications of economic theory and the characteristics of alternative datasets. See also Judd (1997) and McKelvey and McLennan (1996) for an expression of the important role of computational methods in the further development of economic theory.

In Breton, Filar, Haurie, and Schultz (1986), Schultz (1986), Filar, Schultz, Thuijsman, and Vrieze (1991), and Breton (1991), the problem of finding discounted stationary equilibria in the general $n$-person stochastic game is reduced to that of finding a global minimum in a nonlinear 
program with linear constraints. Solving this nonlinear program is equivalent to solving a certain nonlinear system for which it is known that the objective value in the global minimum is zero. But, as is noted in Breton (1991), the convergence of an optimization algorithm to the global optimum is not guaranteed.

In this chapter, an algorithm is proposed that is shown to converge to a stationary equilibrium for a generic $n$-person stochastic game. This algorithm is the first globally convergent algorithm to solve for an equilibrium in an arbitrary $n$-person stochastic game. The algorithm also deals with the problem of equilibrium selection in that it computes the stationary equilibrium selected by the extension of the linear tracing procedure of Harsanyi and Selten (1988) - the stochastic tracing procedure - as defined in the previous chapter.

In Harsanyi and Selten (1988) the tracing procedure is defined for normal form games and for extensive form games with a perfect recall information structure. Algorithms to compute the Nash equilibrium selected by the tracing procedure in normal form games are proposed in van den Elzen and Talman (1999), Herings and van den Elzen (2002), and in Chapter 3. For extensive form games, Harsanyi and Selten (1988) first transform the game into one in standard form and subsequently define the tracing procedure for that class of games. Computation of the Nash equilibrium selected by the tracing procedure in extensive form games is the topic of von Stengel, van den Elzen, and Talman (2001), who invoke the sequence form to calculate such equilibria efficiently.

The algorithm belongs to the class of homotopy methods. The formulation as a differentiable homotopy makes it possible to apply standard path-following techniques that are available in professionally programmed software. This makes implementation on a computer an easy exercise. As a by-product of the proof that the algorithm converges for a generic stochastic game, the result that for a generic stochastic game the stochastic tracing procedure yields a path leading to a unique stationary equilibrium is obtained.

As another by-product of our convergence proof, an extension of a recent result of Haller and Lagunoff (2000) is obtained. Their main result is that the set of stationary equilibria in a stochastic game is generically finite. Here it is found that this number is generically odd.

In Section 6.2 the set of stationary equilibrium strategies is characterized. The definition of the stochastic tracing procedure as proposed in Chapter 5 is given in Section 6.3. In Section 6.4, the properties of the stochastic tracing procedure are studied. It is shown that for almost every stochastic game, the stochastic tracing procedure is formed by a finite union of arcs and loops. Using a well-chosen transformation of variables, the stochastic tracing procedure is described by the zeros of an everywhere differentiable homotopy function in Section 6.5. Section 6.6 discusses the implementation of the homotopy algorithm and provides some numerical results. Section 6.7 summarizes. Section 6.8 contains some lemmas used in the proof to a theorem arising in Section 6.4. 


\subsection{Characterization of stationary equilibria}

Consider a finite discounted stochastic game

$$
\Gamma=\left\langle N, \Omega,\left\{S_{\omega}^{i}\right\}_{(i, \omega) \in N \times \Omega},\left\{u^{i}\right\}_{i \in N}, \pi, \delta\right\rangle,
$$

as defined in Chapter 5. Here, $N=\{1, \ldots, n\}$ is the finite set of players, $\Omega=\left\{\omega_{1}, \ldots, \omega_{z}\right\}$ is the state space containing a finite number of states, and the set $S_{\omega}^{i}=\left\{s_{\omega 1}^{i}, \ldots, s_{\omega m_{\omega}^{i}}^{i}\right\}$ is the finite action set of player $i \in N$ in state $\omega \in \Omega$. Further, $u^{i}: H \rightarrow \mathbb{R}$ is the payoff function of player $i$ and $\pi$ is the transition map $\pi: H \rightarrow \Delta(\Omega)$, where $H=\left\{\left(\omega, s_{\omega}\right) \mid \omega \in \Omega, s_{\omega} \in S_{\omega}\right\}$ and $S_{\omega}=\chi_{i \in N} S_{\omega}^{i}$. If in state $\omega \in \Omega$ the players action choices are $s_{\omega} \in S_{\omega}$, then player $i$ gets an instantaneous payoff of $u^{i}\left(\omega, s_{\omega}\right)$ and the probability that the system jumps to state $\bar{\omega}$ is $\pi\left(\bar{\omega} \mid \omega, s_{\omega}\right)$. Finally, $\delta \in[0,1)$ is a discount factor that is used to discount future payoffs.

Players are allowed to randomize their strategy in each stage of the game. Therefore, let $\Sigma_{\omega}^{i}=\Delta\left(S_{\omega}^{i}\right)$, i.e. the set of all probability distributions over the set $S_{\omega}^{i}$, for all states $\omega \in \Omega$ and all players $i \in N$. The utilities and transition probabilities are extended in the natural von Neumann-Morgenstern way. For further analysis the following notations are introduced: $S^{*}=\bigcup_{(i, \omega) \in N \times \Omega} S_{\omega}^{i}, S=\chi_{(i, \omega) \in N \times \Omega} S_{\omega}^{i}, m^{*}=\left|S^{*}\right|=\sum_{(i, \omega) \in N \times \Omega} m_{\omega}^{i}$, and $m=|S|=$ $\prod_{(i, \omega) \in N \times \Omega} m_{\omega}^{i}$.

Like in the previous chapter, the analysis is focused to stationary strategies, in particular stationary equilibria. Given that the other players play $\rho^{-i}$ and the initial state is $\bar{\omega}$, player $i$ faces the Markov decision problem of maximizing

$$
U^{i}\left(\bar{\omega}, \rho^{-i}, \rho^{i}\right)
$$

over $\rho^{i}{ }^{1}$ subject to

$$
\begin{aligned}
& \rho_{\omega j}^{i} \geq 0 \quad\left(s_{\omega j}^{i} \in S_{\omega}^{i}, \omega \in \Omega\right), \\
& \sum_{s_{\omega j}^{i} \in S_{\omega}^{i}} \rho_{\omega j}^{i}-1=0 \quad(\omega \in \Omega),
\end{aligned}
$$

for all possible initial states $\bar{\omega} \in \Omega$. If the present value of the total expected payoff for a system in state $\omega$ with $k$ transitions remaining is denoted by $\mu_{\omega}^{i}(k)$, the following basic recurrence relation is obtained:

$$
\mu_{\omega}^{i}(k+1)=u^{i}\left(\omega, \rho_{\omega}\right)+\delta \cdot \sum_{\bar{\omega} \in \Omega} \pi\left(\bar{\omega} \mid \omega, \rho_{\omega}\right) \mu_{\bar{\omega}}^{i}(k)
$$

or in vector form

$$
\mu^{i}(k+1)=u^{i}(\rho)+\delta \Pi(\rho) \mu^{i}(k) .
$$

\footnotetext{
${ }^{1}$ Here, the player maximizes over his stationary strategies rather than over his behavioral strategies. But, as already mentioned in Section 5.2 there always exists a best response in stationary strategies when the opponents are playing stationary strategies. Therefore, the maximizing stationary strategy is also a maximizing behavioral strategy. So, it is not the case that non-stationary strategies are not allowed, they only are not subject of selection.
} 
Note that ${ }^{2}$

$$
\begin{aligned}
\mu^{i} & =\lim _{k \rightarrow \infty} \mu^{i}(k)=\lim _{k \rightarrow \infty}\left(\sum_{\ell=0}^{k-1}(\delta \Pi(\rho))^{\ell} u^{i}(\rho)+(\delta \Pi(\rho))^{k} \mu^{i}(0)\right) \\
& =\sum_{\ell=0}^{\infty}(\delta \Pi(\rho))^{\ell} u^{i}(\rho)=[I-\delta \Pi(\rho)]^{-1} u^{i}(\rho)
\end{aligned}
$$

The vector $\mu^{i}$ may be called the vector of present values of player $i$, because each of its elements $\mu_{\omega}^{i}$ is the present value of an infinite number of future expected payoffs discounted by the discount factor $\delta$ with $\omega$ the initial state.

Because interest goes out to the sequential decision process for large $k$ (in fact for $k$ equal to infinity), the present values $\mu_{\omega}^{i}=\lim _{k \rightarrow \infty} \mu_{\omega}^{i}(k)$ are substituted for the quantities $\mu_{\omega}^{i}(k)$ to obtain the recursive relation

$$
\mu_{\omega}^{i}=u^{i}\left(\omega, \rho_{\omega}\right)+\delta \cdot \sum_{\bar{\omega} \in \Omega} \pi\left(\bar{\omega} \mid \omega, \rho_{\omega}\right) \mu_{\bar{\omega}}^{i}
$$

Given that the other players play $\rho^{-i}$, player $i$ maximizes $\mu_{\omega}^{i}$ subject to $\rho_{\omega}^{i} \in \Sigma_{\omega}^{i}$ for all $\omega \in \Omega$.

Because player $i$ maximizes each $\mu_{\omega}^{i}$ for $\omega \in \Omega$, he also maximizes $\sum_{\omega \in \Omega} \mu_{\omega}^{i}$. Given that the other players play $\rho^{-i}$, player $i$ maximizes

$$
\sum_{\omega \in \Omega} \mu_{\omega}^{i}
$$

subject to

$$
\begin{aligned}
& u^{i}\left(\omega, \rho_{\omega}^{-i}, \rho_{\omega}^{i}\right)+\delta \cdot \sum_{\bar{\omega} \in \Omega} \pi\left(\bar{\omega} \mid \omega, \rho_{\omega}^{-i}, \rho_{\omega}^{i}\right) \mu_{\bar{\omega}}^{i}-\mu_{\omega}^{i}=0, \quad(\omega \in \Omega), \\
& \rho_{\omega j}^{i} \geq 0, \quad\left(s_{\omega j}^{i} \in S_{\omega}^{i}, \omega \in \Omega\right), \\
& \sum_{s_{\omega j}^{i} \in S_{\omega}^{i}} \rho_{\omega j}^{i}-1=0, \quad(\omega \in \Omega) .
\end{aligned}
$$

The necessary and sufficient first-order conditions for this maximization problem are

$$
\begin{aligned}
& \alpha_{\omega}^{i}\left\{u^{i}\left(\omega, \rho_{\omega}^{-i}, s_{\omega j}^{i}\right)+\delta \cdot \sum_{\bar{\omega} \in \Omega} \pi\left(\bar{\omega} \mid \omega, \rho_{\omega}^{-i}, s_{\omega j}^{i}\right) \mu_{\bar{\omega}}^{i}\right\}+\beta_{\omega j}^{i}-\gamma_{\omega}^{i}=0, \\
& \beta_{\omega j}^{i} \geq 0, \quad \rho_{\omega j}^{i} \geq 0, \quad \beta_{\omega j}^{i} \rho_{\omega j}^{i}=0, \quad\left(s_{\omega j}^{i} \in S_{\omega}^{i}, \omega \in \Omega\right), \\
& \sum_{s_{\omega j}^{i} \in S_{\omega}^{i}}^{i} \rho_{\omega j}^{i}-1=0, \quad(\omega \in \Omega), \\
& \left.u^{i}\left(\omega, \rho_{\omega}^{-i}, \rho_{\omega}^{i}\right)+\delta \cdot \sum_{\omega}^{i}, \omega \in \Omega\right), \\
& \alpha^{i}\left[I-\delta \Pi\left(\rho^{-i}, \rho^{i}\right)\right]=\mathbb{1} .
\end{aligned}
$$

\footnotetext{
${ }^{2}$ By Hadamard's theorem all eigenvalues of the matrix between the squared brackets have absolute value larger than 0 . Thus zero is not an eigenvalue of that matrix and the inverse exists. Furthermore, the spectral radius of $\delta \Pi(\rho)$ is less than one. Therefore it holds that $[I-\delta \Pi(\rho)]^{-1}=\sum_{k=0}^{\infty} \delta^{k} \Pi(\rho)^{k}$. From this it is easily seen that the value of element $(i, j)$ of the matrix $[I-\delta \Pi(\rho)]^{-1}$ gives the discounted expected number of times that the state is $j$ when $i$ is the initial state and $\rho$ is played.
} 
Here, $\alpha, \beta$ and $\gamma$ are the Lagrange multipliers of the first, second and third set of constraints, and $\mathbb{1 l}$ denotes the vector containing ones only. Then, by multiplying by $\rho_{\omega j}^{i}$ and summing over $j$, for $\rho^{i}$ a best response to $\rho^{-i}$,

$$
\begin{aligned}
& 0=\alpha_{\omega}^{i}\left\{u^{i}\left(\omega, \rho_{\omega}^{-i}, \rho_{\omega}^{i}\right)+\delta \cdot \sum_{\bar{\omega} \in \Omega} \pi\left(\bar{\omega} \mid \omega, \rho_{\omega}^{-i}, \rho_{\omega}^{i}\right) \mu_{\bar{\omega}}^{i}\right\} \\
& +\sum_{s_{\omega j}^{i} \in S_{\omega}^{i}} \beta_{\omega j}^{i} \rho_{\omega j}^{i}-\sum_{s_{\omega j}^{i} \in S_{\omega}^{i}} \gamma_{\omega}^{i} \rho_{\omega j}^{i} \\
& =\alpha_{\omega}^{i} \mu_{\omega}^{i}-\gamma_{\omega}^{i}
\end{aligned}
$$

for all $\omega \in \Omega$. Therefore, since $\alpha^{i}=\left[I-\delta \Pi\left(\rho^{-i}, \rho^{i}\right)\right]^{-1} \mathbb{1}=\frac{1}{1-\delta} \mathbb{1} \gg 0,{ }^{3}$

$$
\frac{\gamma_{\omega}^{i}}{\alpha_{\omega}^{i}}=\mu_{\omega}^{i}
$$

After division by $\alpha_{\omega}^{i}$, the following necessary and sufficient conditions remain, where $\lambda_{\omega j}^{i}$ is defined as the ratio of $\beta_{\omega j}^{i}$ and $\alpha_{\omega}^{i}$

$$
\begin{aligned}
& u^{i}\left(\omega, \rho_{\omega}^{-i}, s_{\omega j}^{i}\right)+\delta \cdot \sum_{\bar{\omega} \in \Omega} \pi\left(\bar{\omega} \mid \omega, \rho_{\omega}^{-i}, s_{\omega j}^{i}\right) \mu_{\bar{\omega}}^{i}+\lambda_{\omega j}^{i}-\mu_{\omega}^{i}=0, \quad\left(s_{\omega j}^{i} \in S_{\omega}^{i}, \omega \in \Omega\right), \\
& \lambda_{\omega j}^{i} \geq 0, \quad \rho_{\omega j}^{i} \geq 0, \quad \lambda_{\omega j}^{i} \rho_{\omega j}^{i}=0, \quad\left(s_{\omega j}^{i} \in S_{\omega}^{i}, \omega \in \Omega\right), \\
& \sum_{s_{\omega j}^{i} \in S_{\omega}^{i}} \rho_{\omega j}^{i}-1=0, \quad(\omega \in \Omega) .
\end{aligned}
$$

Here, $\lambda_{\omega j}^{i}$ is the shadowprice of playing strategy $s_{\omega j}^{i}$, i.e. the disutility from a one-shot deviation at $t=0$ of a marginal increase in the probability $\rho_{\omega j}^{i}$ by which pure strategy $s_{\omega j}^{i}$ is played, and $\mu_{\omega}^{i}$ is the expected payoff of player $i$ when the initial state is $\omega, \rho^{-i}$ is played by his opponents, and player $i$ chooses a best response. The last equality, $\sum_{s_{\omega j}^{i} \in S_{\omega}^{i}} \rho_{\omega j}^{i}-1=0$, makes sure that $\rho_{\omega}^{i}$ is a member of $\Sigma_{\omega}^{i}$ for all $\omega \in \Omega$.

Since for a stationary equilibrium it holds that a strategy-tuple constitutes mutually best responses, it is found that the set of stationary equilibria can be fully characterized by the system of equalities and inequalities in Theorem 6.1 .

Theorem 6.1 A strategy $\rho \in \Sigma$ is a stationary equilibrium if and only if it is part of a solution to

$$
\begin{aligned}
& u^{i}\left(\omega, \rho_{\omega}^{-i}, s_{\omega j}^{i}\right)+\delta \cdot \sum_{\bar{\omega} \in \Omega} \pi\left(\bar{\omega} \mid \omega, \rho_{\omega}^{-i}, s_{\omega j}^{i}\right) \mu_{\bar{\omega}}^{i}+\lambda_{\omega j}^{i}-\mu_{\omega}^{i}=0, \\
& \lambda_{\omega j}^{i} \geq 0, \quad \rho_{\omega j}^{i} \geq 0, \quad \lambda_{\omega j}^{i} \rho_{\omega j}^{i}=0, \quad\left(s_{\omega j}^{i} \in S_{\omega}^{i}, \omega \in \Omega, i \in N\right), \\
& \sum_{s_{\omega j}^{i} \in S_{\omega}^{i}}^{i} \rho_{\omega j}^{i}-1=0, \quad(\omega \in \Omega, i \in N) .
\end{aligned}
$$

\footnotetext{
${ }^{3}$ Since the value of element $(i, j)$ of the matrix $[I-\delta \Pi(\rho)]^{-1}$ gives the discounted expected number of times that the state is $j$ when $i$ is the initial state and $\rho$ is played, the rows sum up to $1 /(1-\delta)$, which is larger than 0 (even larger than 1).
} 
The system above suggests that only one-shot deviations have to be considered. In the next the reason of this surprising phenomenon is intuitively explained.

Suppose the other players play stationary strategies $\rho^{-i}$ and suppose that $\rho^{i}$ is the best stationary response of player $i$. If player $i$ is not able to improve his utility by a deviation to his strategy $\rho^{i}$ in one stage only, then it follows by a backward induction argument that neither finitely many deviations to his strategy will make player $i$ better off. Suppose now that player $i$ can improve his utility by infinitely many changes. Then, by a profit-to-go argument, player $i$ can also increase his payoff by finitely many changes, which is not possible. ${ }^{4}$

This section ends with an example in which it is shown that expected utility in stationary strategies does not hold for the class of stochastic games. This causes a number of technical difficulties for the convergence proof of the algorithm presented later on in the chapter.

Example 6.2 The stochastic game of Figure 6.1 is a game in which there are two states $p_{H}$ and $p_{L}$ and one player. In each state the player can choose between actions $q_{H}$ and $q_{L}$. In the upperleft corner of each square the immediate payoff of the player is displayed. In the lower-right corner the transition probabilities are given. So, if the player chooses $q_{H}$ when the state is $p_{H}$, then he earns 1 today and the next state will be $p_{L}$ with probability 1 . If the player chooses $q_{L}$ when the state is $p_{L}$, then he earns 0 today and the next state will be $p_{H}$ for sure. Future payoffs are discounted by a factor $1 / 2$.
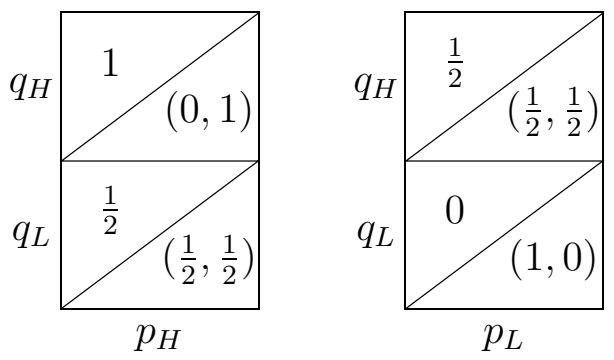

$$
\delta=\frac{1}{2}
$$

Figure 6.1: Example.

Consider the stationary strategies $\rho^{-}=\left(q_{L}, q_{H}\right)$ and $\rho^{+}=\left(q_{H}, q_{L}\right){ }^{5}$ Then $U\left(p_{L}, \rho^{-}\right)=$ $U\left(p_{H}, \rho^{-}\right)=1, U\left(p_{L}, \rho^{+}\right)=2 / 3$ and $U\left(p_{H}, \rho^{+}\right)=4 / 3$. However,

$$
\begin{aligned}
& U\left(p_{H}, \frac{1}{2} \rho^{-}+\frac{1}{2} \rho^{+}\right)=\frac{6}{5} \quad \neq \quad \frac{7}{6}=\frac{1}{2} \cdot 1+\frac{1}{2} \cdot \frac{4}{3}=\frac{1}{2} U\left(p_{H}, \rho^{-}\right)+\frac{1}{2} U\left(p_{H}, \rho^{+}\right), \\
& U\left(p_{L}, \frac{1}{2} \rho^{-}+\frac{1}{2} \rho^{+}\right)=\frac{4}{5} \quad \neq \quad \frac{5}{6}=\frac{1}{2} \cdot 1+\frac{1}{2} \cdot \frac{2}{3}=\frac{1}{2} U\left(p_{L}, \rho^{-}\right)+\frac{1}{2} U\left(p_{L}, \rho^{+}\right) .
\end{aligned}
$$

\footnotetext{
${ }^{4}$ Define $M=\max _{\left(i, \omega, s_{\omega}\right)}\left|u^{i}\left(\omega, s_{\omega}\right)\right|$. Then the maximum payoff a player can earn from time $k$ on is bounded from above by $\delta^{k}\left(1+\delta+\delta^{2}+\ldots\right) M=\delta^{k} /(1-\delta) M$, the so-called maximum 'profit-to-go' value. Suppose player $i$ is able to improve his utility by $\varepsilon$ by means of infinitely many changes. When $k$ grows large the profit-to-go value is at a certain point less then $\varepsilon$ (this is when $k>\log (\varepsilon(1-\delta) / M) / \log (\delta)$ ). This means that the utility improvement by changes until time $k$ (finitely many changes) was positive.

${ }^{5}$ The first argument is the strategy when the state is $p_{H}$ and the second when the state is $p_{L}$.
} 
So, expected utility does not hold.

In the literature on the computation of Nash equilibria in normal form games, a distinction is made between two-player games on the one hand and three or more players on the other hand. For the class of two-player games exact algorithms are possible, because of the bilinear structure of such games (see, for instance the algorithm of Lemke and Howson (1964)). For stochastic games this distinction disappears. The system of equations of Theorem 6.1 is not bilinear, even for two-player games. The paper of Parthasarathy and Raghavan (1981) presents an example of a two-player stochastic game with only rational numbers of payoffs and transition probabilities. The unique Nash equilibrium involves strategies with irrational probabilities. This means that the ordered field property does not hold. A straightforward application of a Lemke-Howson type algorithm to two-player stochastic games is therefore not possible. This shows one more time that the class of stochastic games is considerably more difficult than the class of normal form games.

\subsection{The stochastic tracing procedure}

The linear tracing procedure as presented in Harsanyi and Selten (1988) models a process of convergent expectations by which rational players will come to adopt, and expect each other to adopt, a particular equilibrium as the solution for a given game. Before applying the tracing procedure, every player is assumed to have a subjective probability distribution expressing his expectation about the strategic choices of the other players. Each player is assumed to use the same theory to determine his subjective probability distributions, which makes that all players have the same expectations about the other players. This common subjective probability distribution is called the prior. In the naive Bayesian approach, all players choose best responses to their prior beliefs and would in this way reach a strategy-combination that does not constitute an equilibrium in general. In the linear tracing procedure, the information on the best responses is only gradually fed back into the expectations of the players. As the linear tracing procedure proceeds, both the priors and their best responses will gradually change until both converge to some equilibrium of the game.

In Harsanyi and Selten (1988) the linear tracing procedure is defined for normal form games and for extensive form games with a perfect recall information structure. For a normal form game $\Gamma=\left\langle N,\left\{S^{i}\right\}_{i \in N},\left\{u^{i}\right\}_{i \in N}\right\rangle$ and a prior $p \in \Sigma$, the linear tracing procedure is defined by tracing a curve in the set of Nash equilibria of the games $\Gamma^{t}=\left\langle N,\left\{S^{i}\right\}_{i \in N},\left\{v^{i}(t)\right\}_{i \in N}\right\rangle$ for $t \in[0,1]$, where $v^{i}(t, s)=(1-t) u^{i}\left(p^{-i}, s^{i}\right)+t u^{i}(s)$. For extensive form games, they first transform the game into one in standard form and subsequently define the tracing procedure for that class of games. They did not define the tracing procedure for stochastic games, which are games with instantaneous payoffs and infinite time horizon. Since expected utility in stationary strategies 
does not hold in stochastic games (see Example 6.2) it is not possible to transform this game into one in standard form. The extension of the tracing procedure to the class of stochastic games is far from obvious.

In Chapter 5 four ways to extend the tracing procedure of Harsanyi and Selten to the setting of stochastic games were mentioned. Choices had to be made whether a player holds correlated beliefs within a state or not, and whether a player holds correlated beliefs across time or not. The conclusion in that chapter was that the most natural extension of the linear tracing procedure to stochastic games assumes that beliefs are correlated within states and that they are not correlated across time.

Consider some stochastic game $\Gamma=\left\langle N, \Omega,\left\{S_{\omega}^{i}\right\}_{\omega \in \Omega, i \in N},\left\{u^{i}\right\}_{i \in N}, \pi, \delta\right\rangle$ and some prior $p \in$ $\Sigma$. For every $t \in[0,1]$, the stochastic tracing procedure generates a stationary equilibrium of the stochastic game $\Gamma^{t}=\left\langle N, \Omega,\left\{S_{\omega}^{i}\right\}_{\omega \in \Omega, i \in N},\left\{v^{i}(t)\right\}_{i \in N},\left\{\tilde{\pi}^{i}(t)\right\}_{i \in N}, \delta\right\rangle$, where the instantaneous payoff function $v^{i}(t): \Omega \times S \rightarrow \mathbb{R}$ of player $i$ is defined by

$$
v^{i}\left(t ; \omega, s_{\omega}\right)=(1-t) u^{i}\left(\omega, p_{\omega}^{-i}, s_{\omega}^{i}\right)+t u^{i}\left(\omega, s_{\omega}\right)
$$

and where the transition mapping $\tilde{\pi}^{i}(t)$ is defined by

$$
\tilde{\pi}^{i}\left(t ; \bar{\omega} \mid \omega, s_{\omega}\right)=(1-t) \pi\left(\bar{\omega} \mid \omega, p_{\omega}^{-i}, s_{\omega}^{i}\right)+t \pi\left(\bar{\omega} \mid \omega, s_{\omega}\right)
$$

Note that $\tilde{\pi}^{i}(t)$ is equal for all players when $t=1$. This is consistent with the fact that the tracing procedure should be thought of as a reasoning process. The mapping $\tilde{\pi}^{i}(t)$ should be thought of as what the players think that the transition probabilities are in the stochastic game $\Gamma^{t}$. The expected payoff of player $i$ is easily shown to satisfy the recursive relation

$$
V^{i}(t ; \omega, \rho)=v^{i}\left(t ; \omega, \rho_{\omega}\right)+\delta \cdot \sum_{\bar{\omega} \in \Omega} \tilde{\pi}^{i}\left(t ; \bar{\omega} \mid \omega, \rho_{\omega}\right) V^{i}(t ; \bar{\omega}, \rho) .
$$

The stochastic game $\Gamma^{0}$ corresponds to a trivial stochastic game, where all players believe that all their opponents play with probability 1 according to the prior belief. The stochastic game $\Gamma^{1}$ coincides with the original stochastic game $\Gamma$. A best response against a strategy combination $\rho^{-i} \in \Sigma^{-i}$ in the stochastic game $\Gamma^{t}$ corresponds to a best response against the stationary probability distribution $(1-t)\left[p^{-i}\right]+t\left[\rho^{-i}\right]$ on $S^{-i}$ in the stochastic game $\Gamma$.

The stochastic tracing procedure $\mathcal{S}(\Gamma, p)$ is defined as the set of pairs $(t, \rho)$ for which it holds that $\rho$ is a stationary equilibrium of the stochastic game $\Gamma^{t}$, i.e.

$$
\mathcal{S}(\Gamma, p)=\left\{(t, \rho) \in[0,1] \times \Sigma \mid \rho \text { is a stationary equilibrium of } \Gamma^{t}\right\} .
$$

For a simple proof of the feasibility of the linear tracing procedure for normal form games see Herings (2000) and for the generic well-definedness see Chapter 3. The derivation of such properties for the stochastic tracing procedure is part of present chapter. 


\subsection{Structure of the stochastic tracing procedure}

The size of any stochastic game $\Gamma$ can be characterized by a vector $\eta=\left(n, z,\left(m_{\omega}^{i}\right)_{\omega=1, \ldots, z}^{i=1, \ldots, n}\right)$, specifying the number of players, the number of states, and the number of pure strategies available to a player in a state. The vector $\eta$ is called the size vector of a stochastic game $\Gamma$. For any possible size vector $\eta$, transition map $\pi$ consistent with $\eta$ and discount factor $\delta$, the set $\mathcal{G}(\eta, \pi, \delta)$ of all stochastic games $\Gamma$ possessing $\eta$ as their size vector, having $\pi$ as their transition map, and $\delta$ as discount factor, is called the size class generated by $(\eta, \pi, \delta)$.

Every stochastic game $\Gamma$ in the size class $\mathcal{G}(\eta, \pi, \delta)$ is characterized by a vector $u(\Gamma)$ that contains exactly $n m$ payoffs. A stochastic game $\Gamma$ is identified with the vector $u(\Gamma)$, and the size class $\mathcal{G}(\eta, \pi, \delta)$ is identified with the set of all possible real vectors of size $n m$, that is, with an $n m$-dimensional Euclidean space. It is now possible to define the distance $\varrho\left(\Gamma, \Gamma^{\prime}\right)$ between two stochastic games and the Lebesgue measure of a set of stochastic games.

A given mathematical statement $\mathcal{Z}$ is said to be true for an open set of (almost all, set of generic) stochastic games if, for every possible size class $\mathcal{G}(\eta, \pi, \delta)$, the set $\tilde{\mathcal{G}}(\mathcal{Z})$ of all stochastic games $\Gamma$ in $\mathcal{G}(\eta, \pi, \delta)$ for which the statement $\mathcal{Z}$ is true is open (has full Lebesgue measure, is open and of full Lebesgue measure) with respect to $\mathcal{G}(\eta, \pi, \delta)$.

Fix $\eta, \pi$ and $\delta .{ }^{6}$ Let a subset $B^{*}$ of $S^{*}$ be given with the property that for every player $i$ and for every state $\omega$ there is at least one pure strategy $s_{\omega j}^{i}$ in $B^{*}$, so $B_{\omega}^{i}=B^{*} \cap S_{\omega}^{i} \neq \emptyset$, for every player $i$, for every state $\omega$. Such a set $B^{*}$ is called admissible. The sets $B^{*}$ are used to decompose $\mathcal{S}(\Gamma, p)$ in subsets $\mathcal{S}\left(\Gamma, p, B^{*}\right)$, each having a differentiable manifold structure. The set $\mathcal{S}\left(\Gamma, p, B^{*}\right)$ contains those elements of $\mathcal{S}(\Gamma, p)$ where only strategies in $B^{*}$ are played with positive probability. It is defined by

$$
\begin{aligned}
& \mathcal{S}\left(\Gamma, p, B^{*}\right)=\{(t, \rho) \in \mathcal{S}(\Gamma, p) \\
& s_{\omega j}^{i} \notin B^{*} \Rightarrow \rho_{\omega j}^{i}=0 \\
&\left.s_{\omega j}^{i} \in B^{*} \Rightarrow s_{\omega j}^{i} \in \operatorname{argmax}_{s_{\omega \ell}^{i} \in S_{\omega}^{i}} V^{i}\left(t ; \omega, \rho^{-i}, \rho_{-\omega}^{i}, s_{\omega \ell}^{i}\right)\right\} .
\end{aligned}
$$

It follows that

$$
\mathcal{S}(\Gamma, p)=\bigcup_{B^{*}} \mathcal{S}\left(\Gamma, p, B^{*}\right)
$$

Two sets $\mathcal{S}\left(\Gamma, p, B^{*}\right)$ and $\mathcal{S}\left(\Gamma, p, \bar{B}^{*}\right)$ can only have a point $(t, \rho)$ in common if there is a player $i$ and a strategy $s_{\omega j}^{i}$ such that $\rho_{\omega j}^{i}=0$ and $s_{\omega j}^{i} \in \operatorname{argmax}_{s_{\omega \ell}^{i} \in S_{\omega}^{i}} V^{i}\left(t ; \omega, \rho^{-i}, \rho_{-\omega}^{i}, s_{\omega \ell}^{i}\right)$, so $s_{\omega j}^{i}$ is a best response to $\left(\rho^{-i}, \rho_{-\omega}^{i}\right)$ that is played with probability zero.

\footnotetext{
${ }^{6}$ It is possible to fix only $\eta$, and to define open sets of (almost all, generic sets of) games if these properties hold for every possible size class $\mathcal{G}(\eta)$. All forthcoming proofs can be readily adapted to get results corresponding to these definitions.
} 
To analyze the structure of $\mathcal{S}(\Gamma, p)$ and the sets $\mathcal{S}\left(\Gamma, p, B^{*}\right)$, systems of equalities and inequalities are designed whose solutions characterize these sets. By Theorem 6.1, an element $(t, \rho)$ belongs to $\mathcal{S}(\Gamma, p)$ if and only if it is part of a solution to

$$
\begin{aligned}
& v^{i}\left(t ; \omega, \rho_{\omega}^{-i}, s_{\omega j}^{i}\right)+\delta \cdot \sum_{\bar{\omega} \in \Omega} \tilde{\pi}^{i}\left(t ; \bar{\omega} \mid \omega, \rho_{\omega}^{-i}, s_{\omega j}^{i}\right) \mu_{\bar{\omega}}^{i}+\lambda_{\omega j}^{i}-\mu_{\omega}^{i}=0, \\
& \left(s_{\omega j}^{i} \in S_{\omega}^{i}, \omega \in \Omega, i \in N\right), \\
& \lambda_{\omega j}^{i} \geq 0, \quad \rho_{\omega j}^{i} \geq 0, \quad \lambda_{\omega j}^{i} \rho_{\omega j}^{i}=0, \quad\left(s_{\omega j}^{i} \in S_{\omega}^{i}, \omega \in \Omega, i \in N\right), \\
& \sum_{s_{\omega j}^{i} \in S_{\omega}^{i}} \rho_{\omega j}^{i}-1=0, \quad(\omega \in \Omega, i \in N) \text {. }
\end{aligned}
$$

Given an admissible subset $B^{*}$, define the set $\mathcal{O}\left(\Gamma, p, B^{*}\right)$ as the set of solutions $(t, \rho, \lambda, \mu)$ to the following system of equalities and inequalities:

$$
\begin{aligned}
& v^{i}\left(t ; \omega, \rho_{\omega}^{-i}, s_{\omega j}^{i}\right)+\delta \cdot \sum_{\bar{\omega} \in \Omega} \tilde{\pi}^{i}\left(t ; \bar{\omega} \mid \omega, \rho_{\omega}^{-i}, s_{\omega j}^{i}\right) \mu_{\bar{\omega}}^{i}+\lambda_{\omega j}^{i}-\mu_{\omega}^{i}=0, \\
& \rho_{\omega j}^{i}=0, \quad\left(s_{\omega j}^{i} \in S_{\omega}^{i}, \omega \in \Omega, i \in N\right), \\
& \lambda_{\omega j}^{i}=0, \quad\left(s_{\omega j}^{i} \notin B_{\omega}^{i}, \omega \in \Omega, i \in N\right), \\
& \sum_{s_{\omega j}^{i} \in S_{\omega}^{i}}^{i} \rho_{\omega j}^{i}-1=0, \quad(\omega \in \Omega, i \in N), \\
& \rho_{\omega j}^{i} \geq 0, \quad\left(s_{\omega j}^{i} \in B_{\omega}^{i}, \omega \in \Omega, i \in N\right), \\
& \lambda_{\omega j}^{i} \geq 0, \quad\left(s_{\omega j}^{i} \notin B_{\omega}^{i}, \omega \in \Omega, i \in N\right), \\
& t \geq 0, \\
& 1-t \geq 0 .
\end{aligned}
$$

The fact that for stochastic games the system of equalities and inequalities differs from the case of normal form games, is not the only difficulty. Since expected utility does not hold, properties that are derived from it cannot be used. In particular, it is not even obvious that there is a unique best response to the prior in pure stationary strategies.

Theorem 6.3 implies that $(t, \rho) \in \mathcal{S}\left(\Gamma, p, B^{*}\right)$ if and only if there exists $\lambda \in \mathbb{R}^{m^{*}}$ and $\mu \in \mathbb{R}^{n z}$ such that the equalities (1)-(4) and the inequalities (5)-(8) are satisfied.

Theorem 6.3 Let a stochastic game $\Gamma \in \mathcal{G}(\eta, \pi, \delta)$ and a prior $p \in \Sigma$ be given. For all admissible subsets $B^{*}$ of $S^{*}$, the sets $\mathcal{S}\left(\Gamma, p, B^{*}\right)$ and $\mathcal{O}\left(\Gamma, p, B^{*}\right)$ are $C^{\infty}$ diffeomorphic.

Proof Let $B^{*}$ be an admissible subset of $S^{*}$. For every $i \in N$ and $\omega \in \Omega$ take an element $\bar{s}_{\omega}^{i} \in B^{*}$. Define the function $f:[0,1] \times \Sigma \rightarrow \mathbb{R} \times \mathbb{R}^{m^{*}} \times \mathbb{R}^{m^{*}} \times \mathbb{R}^{n z}$ by $f(t, \rho)=(t, \rho, \lambda, \mu)$, where (in vector form with a slight abuse of notation) $\mu^{i}=\left[I-\delta \cdot \tilde{\Pi}^{i}\left(t ; \rho^{-i}, \bar{s}^{i}\right)\right]^{-1} v^{i}\left(t ; \rho^{-i}, \bar{s}^{i}\right)$ and $\lambda_{\omega \ell}^{i}=\mu_{\omega}^{i}-v^{i}\left(t ; \omega, \rho_{\omega}^{-i}, s_{\omega \ell}^{i}\right)-\delta \cdot \sum_{\bar{\omega} \in \Omega} \tilde{\pi}^{i}\left(t ; \bar{\omega} \mid \omega, \rho_{\omega}^{-i}, s_{\omega \ell}^{i}\right) \mu_{\bar{\omega}}^{i}$. Then $f(t, \rho) \in \mathcal{O}\left(\Gamma, p, B^{*}\right)$ if and only if $(t, \rho) \in \mathcal{S}\left(\Gamma, p, B^{*}\right)$. Note that $f$ defined in this way is a $C^{\infty}$ diffeomorphism.

From Theorem 6.3 it follows that for all $(t, \rho) \in \mathcal{S}\left(\Gamma, p, B^{*}\right)$ there is a unique $\lambda$ and a unique 
$\mu$ such that $(t, \rho, \lambda, \mu) \in \mathcal{O}\left(\Gamma, p, B^{*}\right)$. Vice versa, for all $(t, \rho, \lambda, \mu) \in \mathcal{O}\left(\Gamma, p, B^{*}\right)$ it holds that $(t, \rho) \in \mathcal{S}\left(\Gamma, p, B^{*}\right)$.

The analysis of the system of equalities and inequalities (1)-(8) provides the following result.

Theorem 6.4 There exists an open set of stochastic games and priors $(\Gamma, p) \in \mathcal{G}(\eta, \pi, \delta) \times \Sigma$ with full Lebesgue measure such that for all admissible subsets $B^{*}$ of $S^{*}, \mathcal{S}\left(\Gamma, p, B^{*}\right)$ is a compact 1-dimensional $C^{\infty}$ manifold with boundary. Moreover, a boundary point $(t, \rho)$ of $\mathcal{S}\left(\Gamma, p, B^{*}\right)$ is either

(i) not a boundary point of $\mathcal{S}\left(\Gamma, p, \bar{B}^{*}\right)$ for all $\bar{B}^{*} \neq B^{*}$ and lies in $\{0,1\} \times \Sigma$,

(ii) or, is a boundary point of exactly one $\mathcal{S}\left(\Gamma, p, \bar{B}^{*}\right)$ with $\bar{B}^{*} \neq B^{*}$ and belongs to $(0,1) \times \Sigma$. Moreover, $B^{*}$ and $\bar{B}^{*}$ differ in exactly one element, say $s_{\omega j}^{i}$, for which $\rho_{\omega j}^{i}=0$ and $s_{\omega j}^{i}$ is a best response to $\rho$ in $\Gamma^{t}$.

Proof The proof of this theorem is analogous to the proofs to Theorem 3.2 and 3.3 of Chapter 3 and involves three lemmas. The only thing left to do is to prove those lemmas for the stochastic game situation, which is done in Section 6.8. The inference is then analogously to the proofs of Chapter 3, since it only uses the structural manifold properties of the lemmas.

Lemma 6.8 claims that the set of solutions to (1)-(8) is one-dimensional if the inequalities are strict. Then in Lemma 6.9, it is claimed that the set of solutions to (1)-(8) is zero-dimensional if exactly one of the inequalities is binding. Finally, it is proved in Lemma 6.10 that there is no solution to (1)-(8) with more than one binding inequality.

It follows that $\mathcal{O}\left(\Gamma, p, B^{*}\right)$ is a one-dimensional manifold with boundary, and a point in $\mathcal{O}\left(\Gamma, p, B^{*}\right)$ is a boundary point if and only if exactly one of the inequalities in (5)-(8) holds with equality. By Theorem 6.3 these properties carry over to $\mathcal{S}\left(\Gamma, p, B^{*}\right)$.

It is easily seen that for a boundary point alternative (i) holds when the binding inequality comes from (7) or (8) and that alternative (ii) holds when the binding inequality comes from (5) or (6).

This theorem implies that, for almost every $\Gamma$ and $p$, for all admissible sets $B^{*}$ of $S^{*}$ the set $\mathcal{S}\left(\Gamma, p, B^{*}\right)$ consists of a finite number of smooth arcs and loops. Each arc has two boundary points. The structure of $\mathcal{S}\left(\Gamma, p, B^{*}\right)$ is therefore a simple one; all kinds of complications like bifurcations, spirals, higher dimensional solution sets, diverging behavior, etc. are excluded.

If $\mathcal{S}\left(\Gamma, p, B^{*}\right)$ has a boundary point in $(0,1) \times \Sigma$, then there is a unique admissible subset $\bar{B}^{*} \neq B^{*}$ such that $\mathcal{S}\left(\Gamma, p, \bar{B}^{*}\right)$ has this boundary point in common with $\mathcal{S}\left(\Gamma, p, B^{*}\right)$. The cardinality of $\bar{B}^{*}$ is one less or one greater than the cardinality of $B^{*}$, depending on whether in the common boundary point a strategy in $B^{*}$ is played with probability zero, or whether a strategy not in $B^{*}$ is a best response. The uniqueness of the set $\bar{B}^{*}$ implies that the sets $\mathcal{S}\left(\Gamma, p, B^{*}\right)$ and $\mathcal{S}\left(\Gamma, p, \bar{B}^{*}\right)$ are nicely linked to each other. 
If $\mathcal{S}\left(\Gamma, p, B^{*}\right)$ has a boundary point in $\{0,1\} \times \Sigma$, then this point does not belong to any other set $\mathcal{S}\left(\Gamma, p, \bar{B}^{*}\right)$. This implies that such a boundary point is also a boundary point of $\mathcal{S}(\Gamma, p)$.

Formally, the structure of $\mathcal{S}(\Gamma, p)$ is as follows.

Theorem 6.5 There exists an open set of stochastic games and priors $(\Gamma, p) \in \mathcal{G}(\eta, \pi, \delta) \times \Sigma$ with full Lebesgue measure such that $\mathcal{S}(\Gamma, p)$ is a compact 1-dimensional piecewise $C^{\infty}$ manifold with boundary. The boundary of $\mathcal{S}(\Gamma, p)$ is given by the intersection of $\mathcal{S}(\Gamma, p)$ and $\{0,1\} \times \Sigma$. There is a unique boundary point in $\{0\} \times \Sigma$ which corresponds to a strategy-combination in pure strategies.

Proof The intuition of the proof of the first part of this theorem is given in the text above the theorem. A rigorous proof can be given using the Lemke-Howson argument. A rigorous development of this argument can be found in Chapter 3.

The second part of the theorem, that there is generically a unique boundary point in $\{0\} \times \Sigma$ and that this boundary point is in pure strategies, remains to be shown. Suppose for some player $i$ there is a best response $\bar{\rho}^{i}$ to the prior in mixed strategies. Then for some state $\omega$ player $i$ plays under $\bar{\rho}^{i}$ at least two pure strategies with positive probability mass, say $s_{\omega 1}^{i}$ and $s_{\omega 2}^{i}$. Since $\bar{\rho}^{i}$ is an optimal strategy it is part of a solution to the necessary and sufficient conditions above Theorem 6.1. So,

$$
\lambda_{\omega 1}^{i}=\lambda_{\omega 2}^{i}=0
$$

and

$$
\begin{aligned}
u^{i}\left(\omega, p_{\omega}^{-i}, s_{\omega 1}^{i}\right)+\delta \cdot \sum_{\bar{\omega} \in \Omega} \pi & \left(\bar{\omega} \mid \omega, p_{\omega}^{-i}, s_{\omega 1}^{i}\right) \mu_{\bar{\omega}}^{i}=\mu_{\omega}^{i}= \\
& =u^{i}\left(\omega, p_{\omega}^{-i}, s_{\omega 2}^{i}\right)+\delta \cdot \sum_{\bar{\omega} \in \Omega} \pi\left(\bar{\omega} \mid \omega, p_{\omega}^{-i}, s_{\omega 2}^{i}\right) \mu_{\bar{\omega}}^{i} .
\end{aligned}
$$

It follows that every strategy $\rho^{i}$ with $\rho_{\omega 1}^{i} \geq 0, \rho_{\omega 2}^{i} \geq 0$ and $\rho_{-\{\omega 1, \omega 2\}}^{i}=\bar{\rho}_{-\{\omega 1, \omega 2\}}^{i}$ is a best response to the prior. This contradicts the local finiteness of the number of solutions at $t=0$. Therefore, generically, all best responses are in pure strategies.

Suppose there are two pure best responses $\bar{\rho}^{i}$ and $\hat{\rho}^{i}$ against the prior. Then $\bar{\mu}_{\omega}^{i}=\hat{\mu}_{\omega}^{i}$ for all $\omega \in \Omega$. Take $\rho^{i}=\frac{1}{2} \bar{\rho}^{i}+\frac{1}{2} \hat{\rho}^{i}, \mu^{i}=\bar{\mu}^{i}, \lambda_{\omega j}^{i}=0$ if $\rho_{\omega j}^{i}>0$ and $\lambda_{\omega j}^{i}=\bar{\lambda}_{\omega j}^{i}$ otherwise. Now indeed the complementarity conditions are satisfied and $\rho^{i}$ is a member of $\Sigma^{i}$.

Suppose $\rho_{\omega j}^{i}>0$. Then it holds that $\bar{\rho}_{\omega j}^{i}>0$ or $\hat{\rho}_{\omega j}^{i}>0$; without loss of generality, let $\bar{\rho}_{\omega j}^{i}>0$. Then

$$
\begin{aligned}
0 & =u^{i}\left(\omega, p_{\omega}^{-i}, s_{\omega j}^{i}\right)+\delta \cdot \sum_{\bar{\omega} \in \Omega} \pi\left(\bar{\omega} \mid \omega, p_{\omega}^{-i}, s_{\omega j}^{i}\right) \bar{\mu}_{\bar{\omega}}^{i}-\bar{\mu}_{\omega}^{i}= \\
& =u^{i}\left(\omega, p_{\omega}^{-i}, s_{\omega j}^{i}\right)+\delta \cdot \sum_{\bar{\omega} \in \Omega} \pi\left(\bar{\omega} \mid \omega, p_{\omega}^{-i}, s_{\omega j}^{i}\right) \mu_{\bar{\omega}}^{i}+\lambda_{\omega j}^{i}-\mu_{\omega}^{i} .
\end{aligned}
$$

Suppose $\rho_{\omega j}^{i}=0$. Then it holds that $\bar{\rho}_{\omega j}^{i}=0$ and therefore $\lambda_{\omega j}^{i}=\bar{\lambda}_{\omega j}^{i}$. The equalities above hold again. 
It follows that $\rho^{i}$ is a best response to the prior. This contradicts that, generically, best responses are pure.

For almost every $\Gamma$ and $p$, the set $\mathcal{S}(\Gamma, p)$ consists of a finite number of arcs and loops. Although it is not necessarily the case that these arcs and loops are smooth, the number of nondifferentiabilities is finite at most. Theorem 6.4 implies that all arcs in $\mathcal{S}(\Gamma, p)$ start and end in $\{0,1\} \times \Sigma$. Each such path consists of a finite sequence of smooth $\operatorname{arcs}$ of the sets $\mathcal{S}\left(\Gamma, p, B^{*}\right)$. A loop in $\mathcal{S}(\Gamma, p)$ consists either of a finite sequence (at least two) of differentiable arcs of the sets $\mathcal{S}\left(\Gamma, p, B^{*}\right)$ or is a loop of a set $\mathcal{S}\left(\Gamma, p, B^{*}\right)$. See Figure 6.2 for an impression of the structure of $\mathcal{S}(\Gamma, p)$.

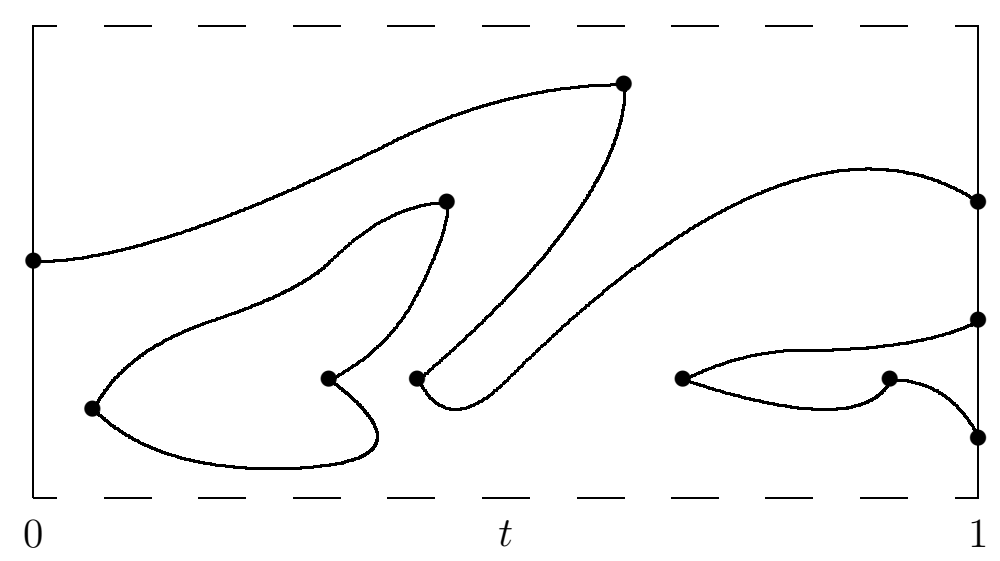

Figure 6.2: The structure of $\mathcal{S}(\Gamma, p)$.

Generically, each player $i$ has a unique best response to the prior for all possible initial states, so generically there is exactly one point of $\mathcal{S}(\Gamma, p)$ that belongs to $\{0\} \times \Sigma$. This point is both a corner point of $\{0\} \times \Sigma$ and a boundary point of $\mathcal{S}\left(\Gamma, p, B^{*}\right)$, where $B^{*}$ consists of the following $n z$ strategies: for each possible initial state and for each player the best response to the prior. Given some initial state $\omega$, the determination of the best response against the prior in state $\omega$ involves the determination of the behavior in all other states $\bar{\omega}$ as well. So the $n z$ independent strategies are found by solving $n$ optimization problems, as opposed to $n z$ independent optimization problems. By Theorem 6.5 this point is the starting point of a uniquely defined arc of $\mathcal{S}(\Gamma, p)$. This arc is the unique feasible path of $\mathcal{S}(\Gamma, p)$ that transforms prior beliefs into (stationary) equilibrium beliefs.

Theorem 6.6 There exists an open set of stochastic games and priors $(\Gamma, p) \in \mathcal{G}(\eta, \pi, \delta) \times \Sigma$ with full Lebesgue measure such that the stochastic tracing procedure is well-defined.

By following the feasible path starting in the unique point $\mathcal{S}(\Gamma, p) \cap(\{0\} \cap \Sigma)$ a stationary equilibrium of the stochastic game $\Gamma$ is found. The set $\mathcal{S}(\Gamma, p) \cap(\{1\} \times \Sigma)$ consists of all 
stationary equilibria of the stochastic game $\Gamma$. Precisely one of these stationary equilibria is an element of the feasible path of $\mathcal{S}(\Gamma, p)$. Any other stationary equilibrium is a boundary point of $\mathcal{S}(\Gamma, p)$ and is therefore part of some arc of $\mathcal{S}(\Gamma, p)$. A moment of reflection makes clear that the remaining stationary equilibria are pairwise connected by arcs from $\mathcal{S}(\Gamma, p)$, and so the number of stationary equilibria is odd.

Theorem 6.7 There exists an open set of stochastic games $\Gamma \in \mathcal{G}(\eta, \pi, \delta)$ with full Lebesgue measure such that the number of stationary equilibria is odd.

The generic oddness of the number of Nash equilibria in normal form games is a well-known result of Rosenmüller (1971), Wilson (1971), and Harsanyi (1973), an alternative proof of which can be found in Chapter 3. The generic finiteness of the number of stationary equilibria in stochastic games is a recent result of Haller and Lagunoff (2000). Theorem 6.7 shows that Haller and Lagunoff's result can be sharpened to oddness.

The observations made so far suggest the following algorithm for the computation of the stationary equilibrium selected by the stochastic tracing procedure in $n$-person stochastic games. Define the admissible set $B^{*}$ that contains for each player $i$ the best response to the prior for all possible initial states, and start with a point $(0, \rho)$ in $\mathcal{S}\left(\Gamma, p, B^{*}\right)$ such that $\rho_{\omega}^{i}$ is a best response of player $i$ to the prior when $\omega$ is the initial state. Theorem 6.5 implies that $B^{*}$ and $\rho$ are uniquely determined. The equalities (1)-(4) belonging to this $B^{*}$ determine at least a part of the feasible path. As long as the inequalities (5)-(8) hold with strict inequality, the set $B^{*}$ remains unchanged. As soon as one of the inequalities from (5) or (6) gets binding, $B^{*}$ has to be changed. When the binding inequality belongs to (5), say $\rho_{\omega j}^{i}=0$ while $s_{\omega j}^{i} \in B^{*}$, strategy $s_{\omega j}^{i}$ has to be removed from $B^{*}$. Obviously, this cannot happen for the starting $B^{*}$. If the binding inequality belongs to (6), say $\lambda_{\omega j}^{i}=0$ while $s_{\omega j}^{i} \notin B^{*}$, strategy $s_{\omega j}^{i}$ has to be added to $B^{*}$. In both situations there is a strategy $s_{\omega j}^{i}$ for which $\rho_{\omega j}^{i}=0$ and $\lambda_{\omega j}^{i}=0$. In general this leads to a kink in the feasible path of the stochastic tracing procedure. This procedure is repeated over and over again, until the inequality (8) is binding and a stationary equilibrium is found. Note that inequality (7) is only binding in the starting point.

\subsection{Smoothing the stochastic tracing procedure}

The previous section presents a method that can be used for the computation of a stationary equilibrium. A potential drawback of that method is that one has to check all the time whether the system of equations used is still appropriate by consulting the inequalities, and if not, one has to switch to a different system of equations. This switching can be a serious problem in terms of computing time. There are $\prod_{(i, \omega) \in N \times \Omega}\left(2^{m_{\omega}^{i}}-1\right)$ different sets $B^{*}$, whereas each one of them may be generated several times in the course of the algorithm. 
The suggestion in Garcia and Zangwill (1981), that is followed in Chapter 3, to formulate one, everywhere differentiable, homotopy by using a well-chosen transformation of variables is followed again. Define, for $\alpha \in \mathbb{R}^{m^{*}}$,

$$
\rho_{\omega j}^{i}(\alpha)=\left[\max \left\{0, \alpha_{\omega j}^{i}\right\}\right]^{2} \quad \text { and } \quad \lambda_{\omega j}^{i}(\alpha)=\left[\max \left\{0,-\alpha_{\omega j}^{i}\right\}\right]^{2} .
$$

After this transformation of variables, the system of equalities and inequalities of Theorem 6.1 becomes

$$
\begin{array}{ll}
v^{i}\left(t ; \omega, \rho_{\omega}^{-i}(\alpha), s_{\omega j}^{i}\right)+\delta \cdot \sum_{\bar{\omega} \in \Omega} \tilde{\pi}^{i}\left(t ; \bar{\omega} \mid \omega, \rho_{\omega}^{-i}(\alpha), s_{\omega j}^{i}\right) \mu_{\bar{\omega}}^{i}+\lambda_{\omega j}^{i}(\alpha)-\mu_{\omega}^{i}=0, & \left(s_{\omega j}^{i} \in S_{\omega}^{i}, \omega \in \Omega, i \in N\right), \\
\lambda_{\omega j}^{i}(\alpha) \geq 0, \quad \rho_{\omega j}^{i}(\alpha) \geq 0, \quad \lambda_{\omega j}^{i}(\alpha) \rho_{\omega j}^{i}(\alpha)=0, \quad\left(s_{\omega j}^{i} \in S_{\omega}^{i}, \omega \in \Omega, i \in N\right), \\
\sum_{s_{\omega j}^{i} \in S_{\omega}^{i}} \rho_{\omega j}^{i}(\alpha)-1=0, \quad(\omega \in \Omega, i \in N) . &
\end{array}
$$

Owing to the proposed transformation of variables, the conditions $\lambda_{\omega j}^{i}(\alpha) \geq 0, \rho_{\omega j}^{i}(\alpha) \geq 0$ and $\lambda_{\omega j}^{i}(\alpha) \rho_{\omega j}^{i}(\alpha)=0$ are trivially satisfied. The set of solutions to (1)-(8) that characterizes the set $\mathcal{O}\left(\Gamma, p, B^{*}\right)$ is up to the transformation of variable equivalent to the set of solutions $(t, \alpha, \mu) \in$ $\mathbb{R} \times \mathbb{R}^{m^{*}} \times \mathbb{R}^{n z}$, with $\alpha_{\omega j}^{i} \geq 0$ if $s_{\omega j}^{i} \in B^{*}$ and $\alpha_{\omega j}^{i} \leq 0$ if $s_{\omega j}^{i} \notin B^{*}$, to

$$
\begin{array}{r}
v^{i}\left(t ; \omega, \rho_{\omega}^{-i}(\alpha), s_{\omega j}^{i}\right)+\delta \cdot \sum_{\bar{\omega} \in \Omega} \tilde{\pi}^{i}\left(t ; \bar{\omega} \mid \omega, \rho_{\omega}^{-i}(\alpha), s_{\omega j}^{i}\right) \mu_{\bar{\omega}}^{i}+\lambda_{\omega j}^{i}(\alpha)-\mu_{\omega}^{i}=0, \\
\left(s_{\omega j}^{i} \in S_{\omega}^{i}, \omega \in \Omega, i \in N\right),
\end{array}
$$

(b) $\quad \sum_{s_{\omega j}^{i} \in S_{\omega}^{i}} \rho_{\omega j}^{i}(\alpha)-1=0, \quad(\omega \in \Omega, i \in N)$,

(c) $\quad t \geq 0$,

(d) $\quad-t+1 \geq 0$.

Notice that the same system of equalities and inequalities can be used, irrespective of the set $B^{*}$. The role of $B^{*}$ is taken over by the sign-combinations of the components of the vector $\alpha$.

Counting equations and unknowns in the system (a)-(d) shows that there is one degree of freedom, and therefore one expects a 1-dimensional solution set. Consider any solution $(t, \alpha, \mu)$ to (a)-(d). When $\alpha_{\omega j}^{i}=0$, then both $\rho_{\omega j}^{i}(\alpha)$ and $\lambda_{\omega j}^{i}(\alpha)$ are zero. This implies that there are exactly two admissible subsets of $S^{*}$ for which the set of (in)equalities (1)-(8) are satisfied. If along a solution curve $\alpha_{\omega j}^{i}$ is increasing while passing zero, then $\rho_{\omega j}^{i}(\alpha)$ gets positive and $B_{\text {new }}^{*}=$ $B_{\text {old }}^{*} \cup\left\{s_{\omega j}^{i}\right\}$. If $\alpha_{\omega j}^{i}$ is decreasing while passing zero, then $\lambda_{\omega j}^{i}(\alpha)$ gets positive and $B_{\text {new }}^{*}=$ $B_{\text {old }}^{*} \backslash\left\{s_{\omega j}^{i}\right\}$. When $\alpha_{\omega j}^{i}$ passes zero, a kink appears in the method proposed in Section 6.4.

The left-hand sides of the equalities (a)-(b) specify the homotopy $\mathcal{H}:[0,1] \times \mathbb{R}^{m^{*}} \times \mathbb{R}^{n z} \rightarrow$ $\mathbb{R}^{m^{*}} \times \mathbb{R}^{n z}$,

$$
\begin{aligned}
& \mathcal{H}(t, \alpha, \mu)= \\
& \left(\begin{array}{cc}
v^{i}\left(t ; \omega, \rho_{\omega}^{-i}(\alpha), s_{\omega j}^{i}\right)+\delta \cdot \sum_{\bar{\omega} \in \Omega} \tilde{\pi}^{i}\left(t ; \bar{\omega} \mid \omega, \rho_{\omega}^{-i}(\alpha), s_{\omega j}^{i}\right) \mu_{\bar{\omega}}^{i}+\lambda_{\omega j}^{i}(\alpha)-\mu_{\omega}^{i}=0 \\
\left(s_{\omega j}^{i} \in S_{\omega}^{i}, \omega \in \Omega, i \in N\right) \\
\sum_{s_{\omega j}^{i} \in S_{\omega}^{i}} \rho_{\omega j}^{i}(\alpha)-1=0 & (\omega \in \Omega, i \in N)
\end{array}\right) .
\end{aligned}
$$


The homotopy function $\mathcal{H}$ is continuously differentiable. The inequalities (c) and (d) are satisfied as the homotopy takes $[0,1]$ as the domain for the variable $t$. Further, $\mathcal{H}$ has the salient feature that its zeros describe the stochastic tracing procedure, $(t, \alpha, \mu) \in \mathcal{H}^{-1}(\{0\})$ if and only if $(t, \rho(\alpha)) \in \mathcal{S}(\Gamma, p)$.

The set $\mathcal{H}^{-1}(\{0\})$ consists of finitely many differentiable arcs and loops. All arcs start and end in $\{0,1\} \times \mathbb{R}^{m^{*}} \times \mathbb{R}^{n z}$. Loops have no points in common with $\{0,1\} \times \mathbb{R}^{m^{*}} \times \mathbb{R}^{n z}$. There is exactly one arc that starts in $\{0\} \times \mathbb{R}^{m^{*}} \times \mathbb{R}^{n z}$ and that ends in $\{1\} \times \mathbb{R}^{m^{*}} \times \mathbb{R}^{n z}$. All other arcs start and end in $\{1\} \times \mathbb{R}^{m^{*}} \times \mathbb{R}^{n z}$ and connect two points inducing stationary equilibria of the stochastic game $\Gamma$. Starting at the unique point $\left(0, \alpha^{0}, \mu^{0}\right) \in \mathcal{H}^{-1}(\{0\})$ at $t=0$ and following the path described by the zeros of $\mathcal{H}$, a point $(1, \tilde{\alpha}, \tilde{\mu}) \in \mathcal{H}^{-1}(\{0\})$ is where the path ends. This point generates the stationary equilibrium $\rho(\tilde{\alpha})$ of $\Gamma$ selected by the stochastic tracing procedure. See Figure 6.3 for an impression of the structure of $\mathcal{H}^{-1}(\{0\})$.

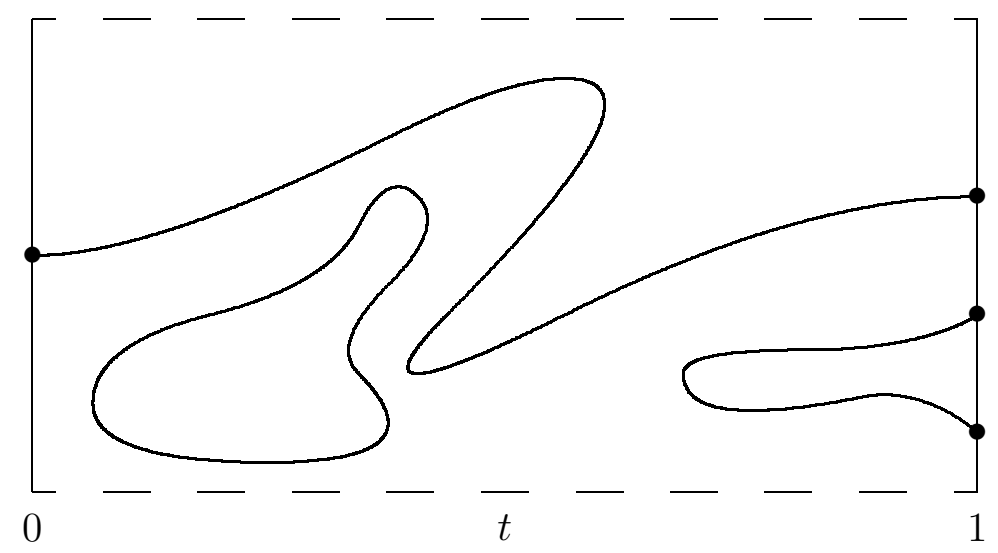

Figure 6.3: The structure of $\mathcal{H}^{-1}(\{0\})$.

The structure of $\mathcal{H}^{-1}(\{0\})$ is even simpler than the one of $\mathcal{S}(\Gamma, p)$. Not only are complications like bifurcations, spirals, higher dimensional solutions sets, diverging behavior, etc., excluded. The arcs and loops in $\mathcal{H}^{-1}(\{0\})$ are differentiable everywhere. It is the transformation of variables that smoothes out the kinks. As a direct consequence, it is possible to calculate the derivative at each point of the feasible path, which makes it possible to follow the path by means of many easily implementable numerical methods, including methods to solve ordinary differential equations.

\subsection{Implementation}

The stochastic game $\Gamma^{0}$ naturally decomposes into $n$ mutually independent and separate Markov decision problems, one for each player. As is shown, generically a Markov decision problem yields a unique optimal pure stationary strategy. The combination of all optimal strategies (for 
each player one) induces the starting point of the algorithm. This point can be determined analytically since there are finitely many pure stationary strategies in each decision problem. For each player only the total discounted payoffs for each of his pure stationary strategies have to be computed and it has to be checked which strategy generates the highest payoff.

Once the starting point is determined, the numerical process starts to follow the homotopypath from that point on. Like in Chapter 3 a number of FORTRAN-subroutines belonging to the software-package HOMPACK are programmed. Again, some subroutines are added to the FIXPDF-subroutines, among which the homotopy function itself as well its Jacobian.

The homotopy function that is implemented is the one as described in Section 6.5 for stochastic games with 2 states, 3 players, with in each state 2 strategies for each player. It may be possible to improve on computing times by rescaling utilities and probabilities, or by using an $\alpha$-transformation with a power different from 2 (but larger than 1, to keep differentiability) or multiplied by a positive scalar different from 1 . An extensive digress on the optimal numerical implementation is beyond the aim of the current analysis.

Within the class of stochastic games with 2 states, 3 players and for each player 2 strategies in each state, five stochastic games and five priors have been generated randomly. All payoffs, transition probabilities, and priors are chosen independently from one another out of the uniform distribution on $[0,1]$. The transition probabilities and the priors are of course normalized to sum up to 1 . The discount factor is fixed at 0.95 . The maximal inaccuracy of our calculation amounts to $10^{-8}$, which means that the 2 -norm of the value of the homotopy function is less than $10^{-8}$ in the computed equilibrium.

\begin{tabular}{lccccc}
\hline & prior 1 & prior 2 & prior 3 & prior 4 & prior 5 \\
\hline game 1 & 1.31 & 1.09 & 1.21 & 1.38 & 1.32 \\
game 2 & 0.82 & 1.37 & 1.27 & 1.43 & 1.10 \\
game 3 & 1.59 & 1.21 & 1.43 & 1.31 & 1.42 \\
game 4 & 1.27 & 1.15 & 1.27 & 1.65 & 0.93 \\
game 5 & 1.54 & 0.77 & 0.88 & 1.26 & 1.48 \\
\hline
\end{tabular}

Table 6.1: Average computation times in seconds.

In Table 6.1 the computation times to compute a stationary equilibrium for each game-prior pair are displayed. ${ }^{7}$ The mean time to compute a stationary equilibrium is 1.26 seconds with a standard deviation of 0.23 seconds. For the games we found on average 1.60 different equilibria with a standard deviation of 0.89 when 5 different priors where used. So, the algorithm is quite fast and multiple equilibria can be found.

\footnotetext{
${ }^{7}$ The computations were performed on a PC operating under Windows 98 with an Intel Pentium II $350 \mathrm{MHz}$ processor and $64 \mathrm{Mb}$ workspace.
} 


\subsection{Summary}

In this chapter it is shown that generically the stochastic tracing procedure consists of a finite number of piecewise smooth arcs and loops, and that the stochastic tracing procedure is welldefined for almost all stochastic games. An everywhere differentiable homotopy function is defined, whose zeros correspond one-to-one to elements of the stochastic tracing procedure, but this set of zeros consists of a finite number of smooth arcs and loops. The unique smooth feasible path from the set of zeros of this homotopy function is followed numerically in order to end up in the stationary equilibrium selected by the stochastic tracing procedure. The resulting algorithm is globally convergent. The numerical performance of the algorithm is presented.

\subsection{Proofs}

For the proofs in this section some notations and definitions from the theory of regular constraint sets are needed (see Chapter 2.4). The notations and definitions used here are similar to those of Section 3.7.

Let a size vector $\eta$, a transition mapping $\pi$, a discount factor $\delta$, and a prior $p \in \Sigma$ be fixed. For any tuple of utility functions $u$ and any admissible subset $B^{*}$, define the functions $g^{B^{*}, u}$ : $\mathbb{R}^{1+2 m^{*}+n z} \rightarrow \mathbb{R}^{2 m^{*}+n z}$ and $h^{B^{*}, u}: \mathbb{R}^{1+2 m^{*}+n z} \rightarrow \mathbb{R}^{m^{*}+2}$ in such a way that $g^{B^{*}, u}$ equals the left-hand side of the equalities (1)-(4) and $h^{B^{*}, u}$ the left-hand side of the inequalities (5)-(8),

$$
g^{B^{*}, u}(t, \rho, \lambda, \mu)=\left(\begin{array}{c}
v^{i}\left(t ; \omega, \rho_{\omega}^{-i}, s_{\omega j}^{i}\right)+\delta \cdot \sum_{\bar{\omega} \in \Omega} \tilde{\pi}^{i}\left(t ; \bar{\omega} \mid \omega, \rho_{\omega}^{-i}, s_{\omega j}^{i}\right) \mu_{\bar{\omega}}^{i} \\
+\lambda_{\omega j}^{i}-\mu_{\omega}^{i} \quad\left(s_{\omega j}^{i} \in S_{\omega}^{i}, \omega \in \Omega, i \in N\right) \\
\rho_{\omega j}^{i} \quad\left(s_{\omega j}^{i} \notin B_{\omega}^{i}, \omega \in \Omega, i \in N\right) \\
\lambda_{\omega j}^{i} \quad\left(s_{\omega j}^{i} \in B_{\omega}^{i}, \omega \in \Omega, i \in N\right) \\
\sum_{s_{\omega j}^{i} \in S_{\omega}^{i}} \rho_{\omega j}^{i}-1 \quad(\omega \in \Omega, i \in N)
\end{array}\right)
$$

and

$$
h^{B^{*}, u}(t, \rho, \lambda, \mu)=\left(\begin{array}{cc}
\rho_{\omega j}^{i} & \left(s_{\omega j}^{i} \in B_{\omega}^{i}, \omega \in \Omega, i \in N\right) \\
\lambda_{\omega j}^{i} & \left(s_{\omega j}^{i} \notin B_{\omega}^{i}, \omega \in \Omega, i \in N\right) \\
t \\
-t+1
\end{array}\right) .
$$

A point $(t, \rho, \lambda, \mu) \in \mathcal{O}\left(\Gamma, p, B^{*}\right)$ is a solution of (1)-(8) if and only if $g^{B^{*}, u}(t, \rho, \lambda, \mu)=0$ and $h^{B^{*}, u}(t, \rho, \lambda, \mu) \geq 0$.

Let the functions $g^{B^{*}}: \mathbb{R}^{1+2 m^{*}+n z} \times \mathbb{R}^{m n} \rightarrow \mathbb{R}^{2 m^{*}+n z}$ and $h^{B^{*}}: \mathbb{R}^{1+2 m^{*}+n z} \times \mathbb{R}^{m n} \rightarrow \mathbb{R}^{m^{*}+2}$ be defined such that $g^{B^{*}}(t, \rho, \lambda, \mu, u)=g^{B^{*}, u}(t, \rho, \lambda, \mu)$ and $h^{B^{*}}(t, \rho, \lambda, \mu, u)=h^{B^{*}, u}(t, \rho, \lambda, \mu)$ 
for all $u \in \mathbb{R}^{m n}$. Figure 6.4 presents the matrix of derivatives of the functions $g^{B^{*}}$ and $h^{B^{*}}$ with respect to all variables, where

$$
\begin{aligned}
& E\left(\left(i^{\prime}, \omega^{\prime}\right), s_{\omega j}^{i}\right)= \begin{cases}1 & \text { if }\left(i^{\prime}, \omega^{\prime}\right)=(i, \omega) \\
0 & \text { otherwise }\end{cases} \\
& \partial_{u^{i^{\prime}}\left(\omega^{\prime}, s_{\omega^{\prime}}^{-i^{\prime}}, s_{\omega^{\prime} j^{\prime}}^{i^{\prime}}\right)} v^{i}\left(t ; \omega, \rho_{\omega}^{-i}, s_{\omega j}^{i}\right)=0 \quad \text { if } s_{\omega j}^{i} \neq s_{\omega^{\prime} j^{\prime}}^{i^{\prime}} \quad \text { for all } s_{\omega^{\prime}}^{-i^{\prime}} \in S_{\omega^{\prime}}^{-i^{\prime}},
\end{aligned}
$$

and

$$
\sum_{s_{\omega}^{-i} \in S_{\omega}^{-i}} \partial_{u^{i}\left(\omega, s_{\omega}^{-i}, s_{\omega j}^{i}\right)} v^{i}\left(t ; \omega, \rho_{\omega}^{-i}, s_{\omega j}^{i}\right)=1 .
$$

The stars $(\star)$ in the matrix need not to be specified for our analysis.

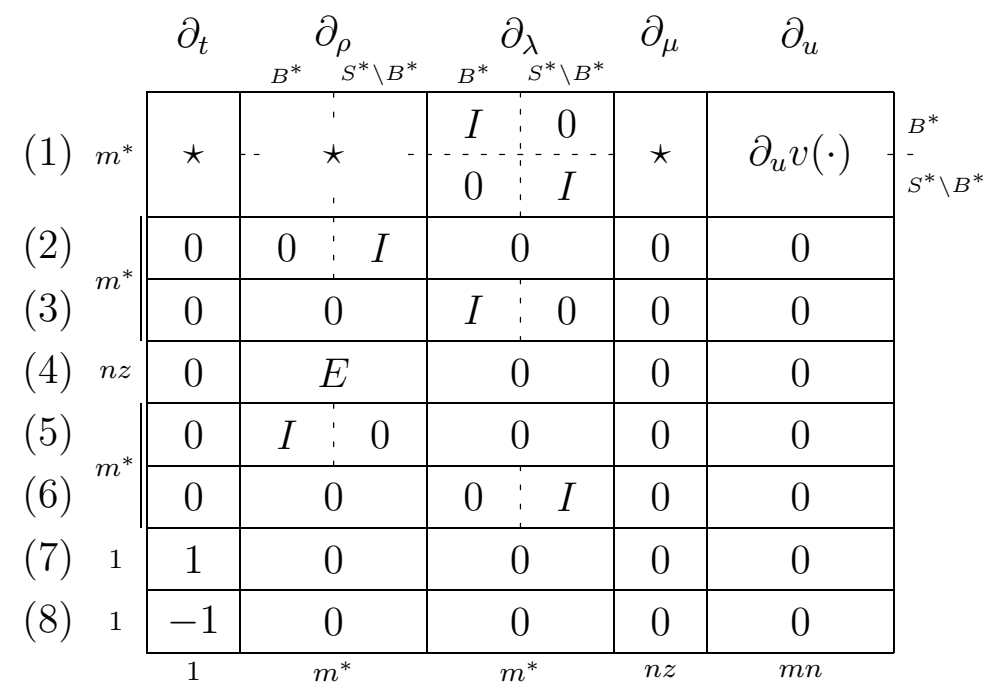

Figure 6.4: The matrix of partial derivatives of $g^{B^{*}}$ and $h^{B^{*}}$.

Each row in Figure 6.4 corresponds to one of the equalities and inequalities in (1)-(8). To make the figure more clear, derivatives with respect to $\rho$ are first taken for $s_{\omega j}^{i} \in B^{*}$. The same applies to the derivatives with respect to $\lambda$ and the ordering of the equalities in (1). From the properties of the matrix, it follows immediately that the matrices $\partial_{u} v$ and $E$ have full row rank.

Lemma 6.8 Let a prior $p \in \Sigma$ and an admissible subset $B^{*}$ of $S^{*}$ be given. Then, for almost all $u, g^{B^{*}, u} \pitchfork\{0\}$.

Proof Consider a point $(\bar{t}, \bar{\rho}, \bar{\lambda}, \bar{\mu}, \bar{u})$ such that $g^{B^{*}}(\bar{t}, \bar{\rho}, \bar{\lambda}, \bar{\mu}, \bar{u})=0$. The matrix of partial derivatives of $g^{B^{*}}$ at $(\bar{t}, \bar{\rho}, \bar{\lambda}, \bar{\mu}, \bar{u})$ is given by the rows (1)-(4) in Figure 6.4. First it is shown that this matrix has full row rank, from which it follows that $g^{B^{*}} \pitchfork\{0\}$.

Since $\partial_{u} v(\cdot)$ has full row rank and the derivative with respect to $u$ in (2)-(4) is zero, it is sufficient to show that the part of the matrix given by (2)-(4) has full row rank. Since the derivative 
with respect to $\lambda$ in (3) has full row rank, whereas the derivative with respect to $\lambda$ in (2) and (4) is zero, it is sufficient to show that the rows in (2) and (4) are independent. The admissibility of $B^{*}$ implies that the derivative with respect to $\rho_{B^{*}}$ in (4) has full row rank. Since, the derivative with respect to $\rho_{B^{*}}$ in (2) is zero, the only thing left to prove is that (2) has independent rows, which is obvious from the derivative with respect to $\rho_{S^{*} \backslash B^{*}}$. Consequently, $g^{B^{*}} \pitchfork\{0\}$.

By the transversality theorem, Theorem 2.26, and since $g^{B^{*}}$ is a $C^{\infty}$ function, it follows that the complement of the set $\left\{u \in \mathbb{R}^{m n} \mid g^{B^{*}, u} \pitchfork\{0\}\right\}$ has Lebesgue measure zero.

Lemma 6.9 Let a prior $p \in \Sigma$ and an admissible subset $B^{*}$ of $S^{*}$ be given. Moreover, let an inequality $j^{\prime} \in\left\{1, \ldots, m^{*}+2\right\}$ be given. Then, for almost all $u,\left(g^{B^{*}, u}, h_{j^{\prime}}^{B^{*}, u}\right) 币\{0\}$.

Proof Consider a point $(\bar{t}, \bar{\rho}, \bar{\lambda}, \bar{\mu}, \bar{u})$ such that $g^{B^{*}}(\bar{t}, \bar{\rho}, \bar{\lambda}, \bar{\mu}, \bar{u})=0$ and $h_{j^{\prime}}^{B^{*}}(\bar{t}, \bar{\rho}, \bar{\lambda}, \bar{\mu}, \bar{u})=0$. The matrix of partial derivatives of $\left(g^{B^{*}}, h_{j^{\prime}}^{B^{*}}\right)$ at $(\bar{t}, \bar{\rho}, \bar{\lambda}, \bar{\mu}, \bar{u})$ is given in Figure 6.4 by the rows (1)-(4) and a single row related to $h_{j^{\prime}}^{B^{*}}$ in (5)-(8). First it is shown that this matrix has full row rank, from which it follows that $\left(g^{B^{*}}, h_{j^{\prime}}^{B^{*}}\right) \pitchfork\{0\}$.

If row $j^{\prime}$ belongs to (6), (7) or (8), then it follows from the derivative with respect to $\lambda_{S^{*} \backslash B^{*}}$ or from the derivative with respect to $t$ that row $j^{\prime}$ has rank 1 . Since all other derivatives in row $j^{\prime}$ are zero, it follows as in the proof of Lemma 6.8 that the rows of (1)-(4) together with row $j^{\prime}$ are independent. Consider the case where row $j^{\prime}$ belongs to (5). Following the first part of the proof of Lemma 6.8, it suffices to prove that (2) and (4) together with row $j^{\prime}$ are independent. Inequality $h_{j^{\prime}}^{B^{*}}$ states that $\rho_{\omega j^{\prime}}^{i} \geq 0, s_{\omega j^{\prime}}^{i} \in B^{*}$, and this inequality is now required to hold with equality. Since $\sum_{s_{\omega j}^{i} \in S_{\omega}^{i}} \rho_{\omega j}^{i}-1=0$, it follows that $\left|B_{\omega}^{i}\right|=\left|B^{*} \cap S_{\omega}^{i}\right|>1$. Then the row related to $s_{\omega j^{\prime}}^{i}$ together with (2) and (4) trivially form an independent set. Consequently, $\left(g^{B^{*}}, h_{j^{\prime}}^{B^{*}}\right) 币\{0\}$. By the transversality theorem it follows that the complement of $\left\{u \in \mathbb{R}^{m n} \mid\left(g^{B^{*}, u}, h_{j^{\prime}}^{B^{*}, u}\right) 历\{0\}\right\}$ has Lebesgue measure zero.

Lemma 6.10 Let a prior $p \in \Sigma$ and an admissible subset $B^{*}$ of $S^{*}$ be given. Moreover, let inequalities $j^{\prime}, j^{\prime \prime} \in\left\{1, \ldots, m^{*}+2\right\}$ with $j^{\prime} \neq j^{\prime \prime}$ be given. Then, for almost all $u$, $\left(g^{B^{*}, u}, h_{j^{\prime}}^{B^{*}, u}, h_{j^{\prime \prime}}^{B^{*}, u}\right) 历\{0\}$.

Proof Consider a point $(\bar{t}, \bar{\rho}, \bar{\lambda}, \bar{\mu}, \bar{u})$ such that $g^{B^{*}}(\bar{t}, \bar{\rho}, \bar{\lambda}, \bar{\mu}, \bar{u})=0, h_{j^{\prime}}^{B^{*}}(\bar{t}, \bar{\rho}, \bar{\lambda}, \bar{\mu}, \bar{u})=0$, and $h_{j^{\prime \prime}}^{B^{*}}(\bar{t}, \bar{\rho}, \bar{\lambda}, \bar{\mu}, \bar{u})=0$. The matrix of partial derivatives of $\left(g^{B^{*}}, h_{j^{\prime}}^{B^{*}}, h_{j^{\prime \prime}}^{B^{*}}\right)$ at $(\bar{t}, \bar{\rho}, \bar{\lambda}, \bar{\mu}, \bar{u})$ is given in Figure 6.4 by the rows (1)-(4) and two rows related to $h_{j^{\prime}}^{B^{*}}$ and $h_{j^{\prime \prime}}^{B^{*}}$ in (5)-(8). First it is shown that this matrix has full row rank, from which it follows that $\left(g^{B^{*}}, h_{j^{\prime}}^{B^{*}}, h_{j^{\prime \prime}}^{B^{*}}\right) 币\{0\}$.

The case where the two rows are not equal to (7) and (8) is similar to the proof of Lemma 6.9. Rows $(7)$ and (8) are not independent. However, they cannot be binding simultaneously, because then it holds that $t=0$ and $t=1$. Consequently, $\left(g^{B^{*}}, h_{j^{\prime}}^{B^{*}}, h_{j^{\prime \prime}}^{B^{*}}\right) 币\{0\}$. It follows that the complement of the set $\left\{u \in \mathbb{R}^{m n} \mid\left(g^{B^{*}, u}, h_{j^{\prime}}^{B^{*}, u}, h_{j^{\prime \prime}}^{B^{*}, u}\right) \pitchfork\{0\}\right\}$ has Lebesgue measure zero. 


\section{Part III}

Application 



\section{Chapter 7}

\section{Dynamic Competition}

In this chapter, which is partly based on Herings, Peeters and Schinkel (2001), the algorithm developed in Chapter 6 is applied to a dynamic entry-and-exit model. For such models, common understanding is that potential entrants will enter into the market up to the point where all excess profits are eroded. Dominant incumbent positions are possible only under specific circumstances, such as the presence of substantial barriers to entry, or when incumbents can credibly threaten to punish rivals with losses upon entry. In this chapter, an equilibrium with market dominance that exists in a simple two-firm model that features neither entry barriers nor punishment strategies is reported on. This equilibrium induces an alternating monopoly - despite the fact that the model also sustains a Cournot duopoly. Even when initially both firms are active in the market, an alternating monopoly reveals itself rather quickly. Moreover, the alternating monopoly equilibrium Pareto dominates the Cournot equilibrium.

\subsection{Introduction}

It is generally claimed that the possibility of rival firms entering into a market served by one or a few large suppliers disciplines the behavior of the incumbent firm(s), provided that the threat of such potential competition is serious enough. The logic is that should the incumbent firm (or firms) make more than a normal profit through the exploitation of its (their) dominant position in the market, entry will take place and intensify actual competition. Incumbent firms, therefore, take into consideration when formulating their market strategy a trade-off between current and future profits. Generally, the higher the former, the lower the latter, and vice versa.

Crucial in the analysis of potential competition and its influence on market performance is the presence of sunk costs. Those are fixed costs that, once laid-out, cannot be recovered again. Examples of sunk costs are costs of schooling of personnel, advertising costs, or the depreciation of assets. To a firm that considers entering a market those entry costs are variable. That is, potential entrants need not make them if they refrain from entry. To a firm in the market, however, 
these costs are truly foregone. This asymmetry creates a barrier to entry to the potential entrant. The higher the sunk costs, the more a firm that considers entry will scratch its head again, since the larger the profit on variable costs needs to be to make up for the lay-out. This, in turn, creates a level of protection from entry to the incumbent firm, that can enjoy modest profits behind its barrier, without triggering an entry process.

The benchmark model used in this type of reasoning is the so-called contestable market model which developed in the 1970s - the condensed reference to which is Baumol, Panzar and Willig (1982). When sunk costs are absent, the model predicts that even when only one firm actually produces something, that firm will be forced to put a quantity on the market that clears at minimum average (i.e. marginal) costs. By the ever present threat of 'hit-and-run' competition, the market performance of a dominant firm is disciplined to the level of a market with perfect competition, which is a situation known to be socially desirable.

The assumption that sunk costs are absent is both crucial to the model and hard to accept. Generally, market entry will require a certain portion of fixed lay-out that is sunk. Consequently, some barriers to entry do exist. This being the case, it may be expected that the contestability of a market is related to the number of potential entrants. Just like the competitiveness of a market increases in the number of actual suppliers, the discipline of the incumbent may well increase in the number of potential competitors that threaten his position.

In the seminal Sherman and Willett (1967), that spawned the debate on the relationship between potential and actual competition, it was shown that this conjecture is not necessarily true. When in a static limit price model potential entrants all behave identically, and simultaneously decide whether to enter the market or not and how much to produce when they do enter, a larger number of them may well convince all of them to stay out of the market. Quite surprisingly then, it may well be that the larger the number of potential entrants, the stronger the position of the incumbent firm. That is, the stronger potential competition, the weaker actual competition, and consequently the lower welfare.

This striking result soon came under attack by adherents to the Chicago School. In Goldberg and Moirao (1973), the Sherman-Willett result was shown to disappear when potential entrants, instead of symmetrically playing a pure strategy 'enter' or 'stay out', would mix between the two. This has the natural interpretation of some firms entering, while others stay out. The mixed strategy Nash equilibrium is characterized by a constant number of expected entrants, simply because an increase of potential entrants leads to a proportional decrease in the probability of each one of them entering. With the expected number of actual entrants as the crucial determinant of the strength of the incumbent's position, no longer does that strength increase in the number of potential entrants. Even though Stiglitz (1981) reproduced and fortified the Sherman-Willett insight, the formalizations of the Chicago view in Grossmann (1981) and the before mentioned Baumol, Panzar and Willig (1982) got the idea stuck that potential competition can serve as a 
substitute for actual competition.

These early contributions to the contestability debate applied quite simple static entry models of the Bain-Sylos-Modigliani-type. ${ }^{1}$ To counter the Chicago consensus, Bernheim (1984) and Dixit and Shapiro (1986) reproduced the argument that potential competition is not necessarily a good for more dynamic models with sequential entry. Several 'Harvardish' surveys of the debate in Dasgupta and Stiglitz (1988), Nti (1989, 2000) and Gilbert (1990) could not compensate the common conception that indeed increased potential competition disciplines the incumbent firms, further fortified by Vives (1988) and Waldman (1991). In Vives (1988), for example, it is shown that in case of sequential entry and quantity setting the number of potential entrants has an influence on the strategy of the incumbent firm to accommodate or not, but never negatively influences social welfare. This common conception shows, for example, clearly from Audretsch, Baumol and Bruke (2001), where the libertarian Austrian ideas on competition policy is favorably revisited as early intuition on current understanding.

In this chapter a dynamic model of entry and exit is considered where a finite number of incumbent firms are pressured by the threat of possible entrance by a finite number of potential entrants. Each period the incumbent firms are in Cournot competition. Additionally to the decision on production quantity, they have to decide whether or not to continue production. Simultaneously and independently, the potential entrants have to make the choice whether or not to enter the market. Since restriction to stationary behavior is made, there is no possibility to use punishments strategies.

Among the questions of interest there are the following. How do the long-run industry dynamics look like? How many firms will be present in the long-run? Is it possible that more firms are present in the long-run when the process starts with less firms? How are prices related to marginal costs and what are the implications for welfare? In order to answer these questions, the analysis is based on the numerical computation of equilibria, as analytical characterizations cannot be obtained.

The dynamic entry-and-exit model is introduced formally in Section 7.2 and strategy and equilibrium concepts are defined. Section 7.3 starts with observing that in each period, given the current market structure, incumbent firms produce the static Cournot quantity. From this observation it follows that the model belongs to the class of finite discounted stochastic games, for which some known results are formulated briefly. In Section 7.4 the model is analyzed for the case when there are two firms in total, there are no sunk costs, and no fixed costs. Where common understanding is that this model will quickly display a long-run Cournot duopoly, a

\footnotetext{
${ }^{1}$ Bain (1949), Sylos-Labini (1962), and Modigliani (1958) formulated the classical theory of limit-pricing. They all posit that the incumbent firm commits to a 'limit quantity', such that the residual demand is insufficient for the entrant - which takes the incumbents quantity as given - to break even. The incumbent can charge the 'limit price' corresponding to the limit quantity and earn profits. In contrast to the classical limit-pricing model, in the model of this chapter firms will act simultaneously and are allowed to play mixed strategies.
} 
different type of equilibrium is found, in which the market is intertemporally shared between the two firms. This alternating monopoly equilibrium Pareto dominates the Cournot equilibrium and is robust in the sense that it remains under positive entry costs and fixed costs. Section 7.5 concludes.

\subsection{The dynamic entry-and-exit model}

This section outlines the model of industry dynamics that is object of study. In each time period, the firms in the model are either an incumbent or a potential entrant. Incumbents produce one homogeneous good with identical cost structures. The market structure at a certain period is given by the information about which of the firms is active in production at that particular period. The instantaneous profits to an incumbent firm in any period depends on the current market structure, the firm's own production quantity and its competitors' production quantities. For a potential entrant the instantaneous profit depends on the entry decision only. Tomorrow's market structure depends on the number of active firms today and all firms' entry and exit decisions today.

There are $n$ firms. The set of firms is denoted by $N=\{1, \ldots, n\}$. The set of market structures is given by $\Omega=\{0,1\}^{n}$, the set of all possible binary arrays of length $n$. For a market structure $\omega \in \Omega$, firm $i$ is a potential entrant when $\omega_{i}=0$ and an incumbent when $\omega_{i}=1$.

In a period, an incumbent firm makes first a decision on production quantity, and second the decision whether to stay active or to exit the industry. Of course, its decisions depend on the market structure, since its instantaneous profit does not only depend on its own production quantity, but also on its competitors' production quantities. When in state $\omega$ a firm $j$ produces quantity $q_{\omega}^{j}$, where $q_{\omega}^{j}=0$ when $\omega_{j}=0$, the instantaneous profit to an incumbent firm $i$ is determined by $u^{i}\left(\omega, q_{\omega}^{i}, q_{\omega}^{-i}\right)=\left(a-b \sum_{j \in N} q_{\omega}^{j}\right) q_{\omega}^{i}-c q_{\omega}^{i}-c_{f}$, where it is assumed that the (inverse) demand curve is linear, that marginal costs $c$ are constant, and that fixed costs are $c_{f}$. The strategy space of an incumbent firm $i$ is denoted by $S_{\omega}^{i}=\mathbb{R}_{+} \times\{$'exit', 'stay' $\}$.

A potential entrant has to decide whether to stay out or to enter the industry conditional on the market structure. When a potential entrant decides to enter it pays entry costs (building the factory) of $c_{e}$ and, consequently, the next period it will be an incumbent. The strategy space of a potential entrant $i$ is denoted by $S_{\omega}^{i}=\{$ 'stay', 'enter' $\}$. At each period, all decisions are made simultaneously and independently.

The transition probabilities $\pi$, which assign to every market structure and decisions taken a new market structure, are deterministic. In the new market situation, again, decisions have to be made, profits are earned and a new market situation will result. Since an infinite horizon model is considered, this procedure goes on and on. Firms maximize total expected profits, where future profits are discounted by a factor $\delta$. 
The firms know the problem data and this knowledge is common knowledge among all firms. Moreover, the initial market structure $\omega^{0}$ at period $k=0$ is common knowledge to the firms. Further, the information structure is of perfect recall which means that each firm remembers all past decisions made by all firms and all past market structures that have occurred.

Note that for this model, in each period a static Cournot game is played. However, contrary to the static Cournot game, the model does not consist of a single decision, but jumps to the next market structure and continues dynamically. In its decisions, a firm does not only take into account the instantaneous profits, but also its opportunities in future stages. Shortly, the main differences to the normal static Cournot conflict situation is that, here, a situation with repeated interaction and the possibility of entry and exit is considered.

The model just described is a problem within the class of stochastic games. Just like in the stochastic game literature we allow firms to randomize their decisions within each time period, so the class of decision possibilities is enlarged from $S_{\omega}^{i}$ to $\Sigma_{\omega}^{i}=\Delta\left(S_{\omega}^{i}\right)$. The instantaneous profits/losses and the transition mapping are extended in the natural Von Neumann-Morgenstern way.

A stationary strategy for firm $i$ is a history and time independent policy which depends on the market structure only. In the sequel, a stationary strategy for firm $i$ will be denoted by $\rho^{i}=\left(\rho_{\omega}^{i}\right)_{\omega \in \Omega}$. If firm $i$ decides on stationary strategy $\rho^{i}$, every time that the system has market structure $\omega$, firm $i$ selects its decision according to $\rho_{\omega}^{i}$.

The Bellman equation which determines the expected discounted value of future net cash flows, when the initial market structure is given by $\omega$ and the firms have stationary strategies $\rho=\left(\rho^{i}\right)_{i \in N}$, is given by

$$
U^{i}(\omega, \rho)=u^{i}\left(\omega, \rho_{\omega}\right)+\delta \cdot \sum_{\bar{\omega} \in \Omega} \pi\left(\bar{\omega} \mid \omega, \rho_{\omega}\right) U^{i}(\bar{\omega}, \rho) .
$$

A stationary strategy-combination is a stationary equilibrium if it is a Nash equilibrium in stationary strategies.

From here on, the analysis is restricted to the class of stationary strategies and thereby to the class of stationary equilibria. In addition to the motivations for restricting attention to stationary strategies given by Maskin and Tirole (2001), which were mentioned in Chapter 5, stationarity of the behavior strategies of the firms, coupled with our state space, excludes the possibility to punish the rival firm. Consequently, none of the results obtained below relies on any form of punishment strategies by construction.

\subsection{Finite stochastic game model}

Due to the stationarity assumed and thereby the absence of the possibility to use punishment strategies, in equilibrium, all active firms produce the static Cournot quantity. Hence, when there 
are $m_{\omega}=\#\left\{i \in N \mid \omega_{i}=1\right\}$ firms active in the market $\left(m_{\omega} \in\{0, \ldots, n\}\right)$, each incumbent produces the static Cournot quantity given by

$$
q_{m_{\omega}}^{C}=\frac{(a-c)}{\left(m_{\omega}+1\right) b},
$$

and the instantaneous profit to each of the $m_{\omega}$ active firms is

$$
u_{m_{\omega}}^{C}=\frac{(a-c)^{2}}{\left(m_{\omega}+1\right)^{2} b}-c_{f} .
$$

For this reason we can restrict the strategy space of incumbent firms to their dynamic decision whether to stay active or to exit, i.e. $S_{\omega}^{i}=\{$ 'exit', 'stay' $\}$. The model fits in the class of finite discounted stochastic games as firstly defined in Shapley (1953) and studied in Chapter 5 and Chapter 6, since all firms have now a finite number of pure strategies in all possible market structures. For the finite discounted stochastic game stationary equilibria exist, as follows from Fink (1964), Takahashi (1964), and Sobel (1971). Moreover, their number is generically finite, as is shown in Haller and Lagunoff (2000), and odd as is shown in Chapter 6. From Chapter 6 it also follows that the set of stationary equilibria can be characterized by a system of polynomial equations and inequalities, as stated in Theorem 6.1 .

Theorem 7.1 A stationary strategy-combination $\rho=\left(\rho^{i}\right)_{i \in N}$ is a stationary equilibrium for all possible initial market structures $\omega \in \Omega$ if and only if there are $\lambda=\left(\lambda^{i}\right)_{i \in N}$ and $\mu=\left(\mu^{i}\right)_{i \in N}$ such that

$$
\begin{aligned}
& u^{i}\left(\omega, s_{\omega j}^{i}, \rho_{\omega}^{-i}\right)+\delta \cdot \sum_{\bar{\omega} \in \Omega} \pi\left(\bar{\omega} \mid \omega, s_{\omega j}^{i}, \rho_{\omega}^{-i}\right) \mu_{\bar{\omega}}^{i}+\lambda_{\omega j}^{i}-\mu_{\omega}^{i}=0, \\
& \left(s_{\omega j}^{i} \in S_{\omega}^{i}, \omega \in \Omega, i \in N\right), \\
& \sum_{s_{\omega j}^{i} \in S_{\omega}^{i}} \rho_{\omega j}^{i}-1=0, \quad(\omega \in \Omega, i \in N), \\
& \lambda_{\omega j}^{i} \geq 0, \quad \rho_{\omega j}^{i} \geq 0, \quad \lambda_{\omega j}^{i} \rho_{\omega j}^{i}=0, \quad\left(s_{\omega j}^{i} \in S_{\omega}^{i}, \omega \in \Omega, i \in N\right) .
\end{aligned}
$$

As for each firm its opponents are identical - except perhaps for their position in the market the analysis is further restricted to the class of anonymous stationary strategies.

Definition 7.2 A stationary strategy $\rho^{i}=\left(\rho_{\omega}^{i}\right)_{\omega \in \Omega}$ is anonymous if $m_{\omega}=m_{\bar{\omega}}$ and $\omega_{i}=\bar{\omega}_{i}$ implies $\rho_{\omega}^{i}=\rho_{\bar{\omega}}^{i}$.

In words, anonymity yields that firms do only base their decision on the number of incumbents present rather than on which firms are present. Therefore the state space $\Omega=\{0,1\}^{n}$ can be reduced to $\Omega^{a}=\{0,1\} \times\{0, \ldots, n-1\}$. A generic element of $\Omega^{a}$ will be noted by $(b, m)$. The first element $b$ is a boolean which tells to each firm whether it is present $(b=1)$ or not $(b=0)$. The second element $m$ gives the number of opponents present $(m \in\{0, \ldots, n-1\}$ ). 
When some firm observes market structure $(1, m)$, it knows that it is an incumbent and that exactly $m$ of its $n-1$ opponents are also incumbents that also observe market structure $(1, m)$; the remaining $(n-1)-m$ firms are potential entrants that observe market structure $(0, m+1)$. Similarly, when some firm observes market structure $(0, m)$, it knows that it is a potential entrant just like $(n-1)-m$ other firms; the remaining $m$ firms are incumbents that observe market structure $(1, m-1)$.

Definition 7.3 A stationary equilibrium that satisfies the anonymity property is an anonymous stationary equilibrium.

As all firms are identical - again, except for their position in the market - symmetric anonymous stationary strategies are considered.

Definition 7.4 An anonymous stationary strategy $\rho^{i}=\left(\rho_{(b, m)}^{i}\right)_{(b, m) \in \Omega^{a}}$ is symmetric when $\rho_{(b, m)}^{i}=$ $\rho_{(b, m)}^{k}$ for all $i, k \in N$.

Intuitively, symmetry in addition to anonymity yields that incumbent firms behave the same when the same number of active opponents is observed, and that potential entrants behave the same when the same observation about the market structure is made.

Definition 7.5 An anonymous stationary equilibrium that satisfies the symmetry property is a symmetric anonymous stationary equilibrium.

Trivially, a symmetric anonymous stationary equilibrium is determined by one single symmetric anonymous stationary strategy which is a best response to itself. Using the knowledge on the instantaneous profits, the following theorem gives a characterization of the set of symmetric anonymous stationary equilibria.

Theorem 7.6 A symmetric anonymous stationary strategy $\rho$ constitutes a symmetric stationary equilibrium for all initial market structures $(b, m) \in \Omega^{a}$ if and only if there are $\lambda$ and $\mu$ such that

$$
\begin{aligned}
& u_{m+1}^{C}+\delta \cdot \sum_{\bar{m}} \pi\left((1, \bar{m}) \mid(1, m), \rho_{(1, m)}, \rho_{(0, m+1)}, s_{(1, m)}^{\text {stay }}\right) \mu_{(1, \bar{m})}+\lambda_{(1, m)}^{\text {stay }}-\mu_{(1, m)}=0, \\
& u_{m+1}^{C}+\delta \cdot \sum_{\bar{m}} \pi\left((0, \bar{m}) \mid(1, m), \rho_{(1, m)}, \rho_{(0, m+1)}, s_{(1, m)}^{\text {exit }}\right) \mu_{(0, \bar{m})}+\lambda_{(1, m)}^{\text {exit }}-\mu_{(1, m)}=0, \\
& \lambda_{(1, m)}^{\text {stay }} \geq 0, \quad \rho_{(1, m)}^{\text {stay }} \geq 0, \quad \lambda_{(1, m)}^{\text {stay }} \rho_{(1, m)}^{\text {stay }}=0, \\
& \lambda_{(1, m)}^{\text {exit }} \geq 0, \quad \rho_{(1, m)}^{\text {exit }} \geq 0, \quad \lambda_{(1, m)}^{\text {exit }} \rho_{(1, m)}^{\text {exit }}=0, \\
& \rho_{(1, m)}^{\text {stay }}+\rho_{(1, m)}^{\text {exit }}-1=0,
\end{aligned}
$$

for all $(1, m) \in \Omega^{a}$, and

$$
\begin{aligned}
& 0+\delta \cdot \sum_{\bar{m}} \pi\left((0, \bar{m}) \mid(0, m), \rho_{(1, m-1)}, \rho_{(0, m)}, s_{(0, m)}^{\text {stay }}\right) \mu_{(0, \bar{m})}+\lambda_{(0, m)}^{\text {stay }}-\mu_{(0, m)}=0, \\
& -c_{e}+\delta \cdot \sum_{\bar{m}} \pi\left((1, \bar{m}) \mid(0, m), \rho_{(1, m-1)}, \rho_{(0, m)}, s_{(1, m)}^{\text {enter }}\right) \mu_{(1, \bar{m})}+\lambda_{(0, m)}^{\text {enter }}-\mu_{(0, m)}=0,
\end{aligned}
$$




$$
\begin{array}{ll}
\lambda_{(0, m)}^{\text {stay }} \geq 0, \quad \rho_{(0, m)}^{\text {stay }} \geq 0, & \lambda_{(0, m)}^{\text {stay }} \rho_{(0, m)}^{\text {stay }}=0, \\
\lambda_{(0, m)}^{\text {enter }} \geq 0, \quad \rho_{(0, m)}^{\text {enter }} \geq 0, & \lambda_{(0, m)}^{\text {enter }} \rho_{(0, m)}^{\text {enter }}=0 \\
\rho_{(0, m)}^{\text {stay }}+\rho_{(0, m)}^{\text {enter }}-1=0, &
\end{array}
$$

for all $(0, m) \in \Omega^{a}$.

Theorem 7.7 A symmetric anonymous stationary equilibrium exists.

Proof Sketch of proof: (1) Let $\sigma: \Sigma^{i} \rightarrow \Sigma^{i}$ be the best response correspondence. Apply Kakutani's fixed point theorem. (2) Prove by contradiction that there is a best response that only depends on $\Omega^{a}$, i.e. there is no improvement in $\Omega$.

Since in each market structure the instantaneous payoff to a firm is positive when $u_{n}^{C} \geq 0$ and entry costs can be earned back when $c_{e} \leq \frac{\delta}{1-\delta} u_{n}^{C}$ the following theorem is quite trivial.

Theorem 7.8 If $u_{n}^{C} \geq 0$ and $c_{e} \leq \frac{\delta}{1-\delta} u_{n}^{C}$, then always 'enter' when absent and always 'stay' when present is a symmetric anonymous stationary equilibrium.

Although the equilibrium in last theorem obviously exists, it is not necessarily the unique one. In the next section we will see that there might exist other equilibria, in fact even other symmetric anonymous stationary equilibria.

\subsection{When there are two firms}

This section reports on a surprising result in the simplest possible dynamic model of competition with entry and exit. There are no sunk costs, hence no barriers to entry, incumbents are in Cournot competition, entry and exit decisions take the form of mixed strategies, and there is perfect and complete information. Moreover, the analysis is limited to symmetric anonymous stationary strategies, thereby ruling out punishment strategies. Whereas one would expect that the model will quickly display a long-run Cournot duopoly, a different type of equilibrium is found, namely one in which the market is intertemporally shared between the two firms. When the firms coordinate on this equilibrium, the probability that this industry structure of alternating monopoly is observed converges quickly to one, for all reasonable discount factors. Moreover, this equilibrium Pareto-dominates the Cournot equilibrium. Both firms are able to obtain higher profits by dividing the market intertemporally between them. This provides an interesting alternative to geographical market division. It has so far been overlooked in the literature, yet it potentially poses a threat to competitive markets, and as such it possibly is a subject of antitrust policy concern. 


\subsubsection{The model}

Suppose there are only two firms, no fixed costs, no entrance costs and discount factor 0.95. So, $n=2, c_{f}=0, c_{e}=0$, and $\delta=0.95$. In Figure 7.1 the stochastic game corresponding to this specific situation is depicted, where it is assumed, without loss of generality, that the common factor in the instantaneous profits for all $m$ (here, $m$ equals 1 or 2 ) is unity. That is, $\frac{(a-c)^{2}}{b}=1$. This can simply be justified by the choice of units. A firm choosing the strategy 'in' will produce
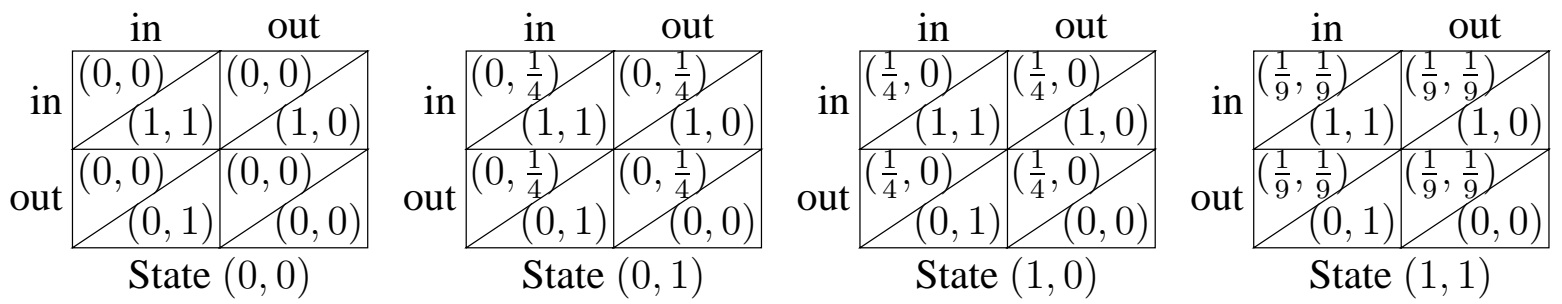

Figure 7.1: The dynamic entry-and-exit model as a stochastic game.

in the next period, and a firm choosing strategy 'out' will be out of production in the next period. The instantaneous profits of the firms are given by the tuple in the upper-left part of the cells. The lower-right part of the cell indicates the next state, reached with probability one - $(1,1)$ means for example both firms in, and (0,1) firm 1 out, firm 2 in.

The set of symmetric anonymous stationary equilibria can be characterized by a system of polynomial equalities and inequalities as is shown in Theorem 7.6. Since analytically solving these kind of polynomial systems is often not possible, resort to numerical methods is needed. Recently, some progress towards this end has been made for finite discounted stochastic games in general in Herings and Peeters (2000) (see also Chapter 6), in which an algorithm to compute stationary equilibria for this class of games is developed and is shown to converge almost surely. The algorithm belongs to the class of homotopy methods and is implemented by including a number of subroutines into HOMPACK, a software-package written in FORTRAN to do homotopycontinuation calculations. All equilibria of this model arising in the sequel can be easily checked to be equilibria by substituting the relevant variables into the system of polynomial (in)equalities of Theorem 7.6.

\subsubsection{Alternating monopoly}

Conventional wisdom quickly leads to the claim that the market dynamics modeled above will typically result in the two firms sharing the market forever. After all, in the Cournot duopoly situation, there is a substantial profit over and above the entry costs - here assumed to be zero - for each firm. Moreover, there is no dynamic limit pricing, which would be fruitless in the absence of barriers to entry, nor do firms deploy punishment strategies, as they cannot by construction. 
And indeed it is the case that 'always stay when active' and 'always enter when not active' or, alternatively, 'produce in the next period no matter what the current market structure is', is a symmetric anonymous stationary equilibrium, as is claimed in Theorem 7.8.

Table 7.1 displays, these equilibrium strategies for each firm in each state, as well as the expected total discounted profits to the firms by coordinating on this equilibrium for each possible initial state. Irrespective of whether initially neither firm, both firms, or one of the two firms is in

\begin{tabular}{c||c|c|c|c||c|c}
\multicolumn{1}{c||}{} & \multicolumn{4}{c||}{ equilibrium strategies } & \multicolumn{2}{c}{$\begin{array}{c}\text { equilibrium } \\
\text { payoffs }\end{array}$} \\
\cline { 2 - 6 } $\begin{array}{c}\text { (initial) } \\
\text { state }\end{array}$ & in & out & in & out & firm 1 & firm 2 \\
\cline { 2 - 6 }$(0,0)$ & 1.0000 & 0.0000 & 1.0000 & 0.0000 & 2.1111 & 2.1111 \\
$(0,1)$ & 1.0000 & 0.0000 & 1.0000 & 0.0000 & 2.1111 & 2.3611 \\
$(1,0)$ & 1.0000 & 0.0000 & 1.0000 & 0.0000 & 2.3611 & 2.1111 \\
$(1,1)$ & 1.0000 & 0.0000 & 1.0000 & 0.0000 & 2.2222 & 2.2222
\end{tabular}

Table 7.1: Stationary Cournot equilibrium with two active firms.

the market, each will produce in the next period with probability 1 . There is only a slightly higher payoff for the firm that happened to be first incumbent, enjoying an instantaneous monopoly profit for a single period, when the initial market structure featured a monopoly.

Apart from this known equilibrium, however, a different stationary equilibrium turns out to exist, with a fundamentally different nature. Table 7.2 displays for this equilibrium its diagnostics. In this equilibrium, when none of the firms produces today, each will produce tomorrow

\begin{tabular}{c||c|c|c|c||c|c}
\multicolumn{1}{c||}{\multirow{2}{*}{$\begin{array}{c}\text { (initial) } \\
\text { state }\end{array}$}} & \multicolumn{3}{c||}{ equilibrium strategies } & \multicolumn{2}{c}{$\begin{array}{c}\text { equilibrium } \\
\text { payoffs }\end{array}$} \\
\cline { 2 - 6 } & in & out & in & out & firm 1 & firm 2 \\
\hline$(0,0)$ & 0.9306 & 0.0694 & 0.9306 & 0.0694 & 2.3055 & 2.3055 \\
$(0,1)$ & 1.0000 & 0.0000 & 0.0000 & 1.0000 & 2.4359 & 2.5641 \\
$(1,0)$ & 0.0000 & 1.0000 & 1.0000 & 0.0000 & 2.5641 & 2.4359 \\
$(1,1)$ & 0.9306 & 0.0694 & 0.9306 & 0.0694 & 2.4166 & 2.4166
\end{tabular}

Table 7.2: Stationary equilibrium with alternating monopoly.

with probability 0.9306 . This means that with probability $(1-0.9306)^{2}=0.0048$ no firm will enter, with probability 0.1292 exactly one - the probabilities are equally split over the two possible situations - and with probability 0.8660 two firms will be active tomorrow. Similarly, when in a certain period both firms are active, the next period all market structures can be reached with positive probability. However, once a firm is the only firm in the market today it will with certainty stop producing tomorrow, whereas the opponent firm that is not in the market today will be for sure one period later. The market will, in other words, display an alternating monopoly. 
This dynamic stationary market equilibrium of alternating monopoly is, to our knowledge, nowhere mentioned in the literature. ${ }^{2}$ Yet, it is not difficult to come up with examples of markets that seem indeed to display the type of production planning of the alternating monopolists, and that have the characteristics of our simple model. Television stations, for example, often alternate their prime shows, such as news services and sit-coms, intertemporally, so that they do not compete for viewers in the same time slot. Summer events, such as open air pop festivals, or local fancy fairs, are planned in different weeks of the season. And similarly, major sports games, particularly important matches such as finals, are planned not to overlap - both within one and the same sport, but particularly also over different sports. Likewise, it has been observed that large companies of relatively homogeneous commodities that are only substantially differentiated by application of marketing methods, such as cola, tend to alternate their advertising campaigns. None of these alternations in production involve any major entry or exit costs, if any at all. Next to geographical market division, therefore, intertemporal market division can well be a form of economic market division that reduces competition, and thus welfare.

\subsubsection{Speed of convergence to and robustness of alternating monopoly}

Having established its existence, the natural question to ask is how fast the dynamics converges to the alternating monopoly and how robust the alternating monopoly equilibrium is. One approach to this question is to consider how long it takes before an alternating monopoly reveals itself, when both firms coordinate on that equilibrium. A first observation towards this is on the role of the discount factor $\delta$. For values of $\delta$ large enough, the alternating monopoly equilibrium exists and can be calculated. However, when firms become sufficiently impatient, it no longer pays to divide the market intertemporally, and the equilibrium disappears. This critical value of $\delta$ is equal to 0.80 , which corresponds to an interest rate of $25 \%$ per period. For all higher discount factors, an equilibrium industry structure characterized by an alternating monopoly can be found.

The likelihood of indeed observing an alternating monopoly can subsequently be studied as follows. Once the industry is in an alternating monopoly situation in a certain period, it will stay in that equilibrium forever, where at even periods the one firm is active and at odd periods the other. When the market structure is not a monopoly in a certain period, there is a positive probability that it will end up in a monopoly next period. Consequently, the cumulative probability of an alternating monopoly increases in time.

To see the speed with which this likelihood converges to one, consider the Markov process induced by the alternating monopoly equilibrium. Its transition probabilities are illustrated graph-

\footnotetext{
${ }^{2}$ Note that equilibrium strategies with alternating actions that exist in repeated games - such as the infinitely repeated prisoners' dilemma, where the asymmetrically alternating strategy 'defect-cooperate-defect-cooperate-etc.' is a Nash equilibrium - all rely on the Folk Theorem in that they require punishment strategies, which are here absent by construction. $C f$. Chapter 5 of Fudenberg and Tirole (1995).
} 


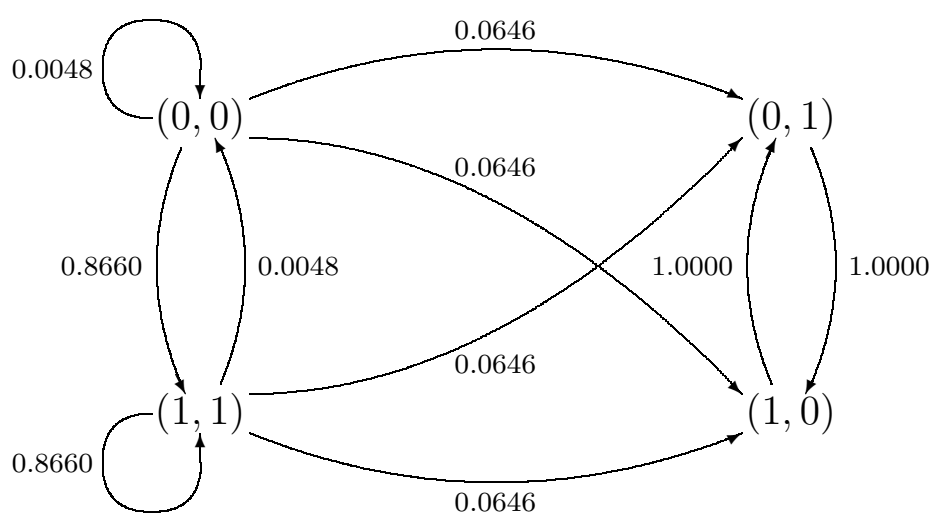

Figure 7.2: Transition-probabilities in market structure.

ically in Figure 7.2 for $\delta=0.95$. These state-transitions can be caught in the transition-matrix $P$ defined as

$$
P=\left[\begin{array}{llll}
0.0048 & 0.0646 & 0.0646 & 0.8660 \\
0.0000 & 0.0000 & 1.0000 & 0.0000 \\
0.0000 & 1.0000 & 0.0000 & 0.0000 \\
0.0048 & 0.0646 & 0.0646 & 0.8660
\end{array}\right] .
$$

The probability of having a certain market structure after two periods knowing the current market structure is given in the matrix $P^{2}$. When the current market structure is $\omega$, the probability that the market structure is $\bar{\omega}$ after $k$ periods is given by the value of the matrix cell in row $\omega$ and column $\bar{\omega}$ of the matrix $P^{k}$. Regardless of what market structure is initially prevailing, therefore, the probability that an alternating monopoly is found after $k$ periods is at least equal to $P^{k}(1,2)+P^{k}(1,3) .^{3}$

For all periods up to 50 these probabilities have been computed. The resulting data is displayed in Figure 7.3, for various values of $\delta$. Clearly, except for values of $\delta$ close to 0.80 , within some 50 periods the probability of an alternating monopoly rapidly converges to one. In fact, for $\delta=0.90$ the cumulative probability of an alternating monopoly is larger than 0.99 after exactly 50 periods. For $\delta=0.95$ this is the case after 34 periods, for $\delta=0.99$ after 27 periods, but even when $\delta=0.85$, it takes less than 100 periods for the cumulative probability of observing alternating monopoly to reach 0.99 .

There remains the question how reasonable it is to assume that coordination on the alternating monopoly equilibrium will happen. Towards answering this, it should be noted that in any game with multiple equilibria a similar question arises for each particular equilibrium. Hence, for that matter, the alternating monopoly case is no less likely than the Cournot equilibrium. However, if

\footnotetext{
${ }^{3}$ Naturally, it is also at least equal to $P^{k}(4,2)+P^{k}(4,3)$. Moreover, computations showed that, in absence of entry costs, $P(1,2)=P(1,3)=P(4,2)=P(4,3)$.
} 


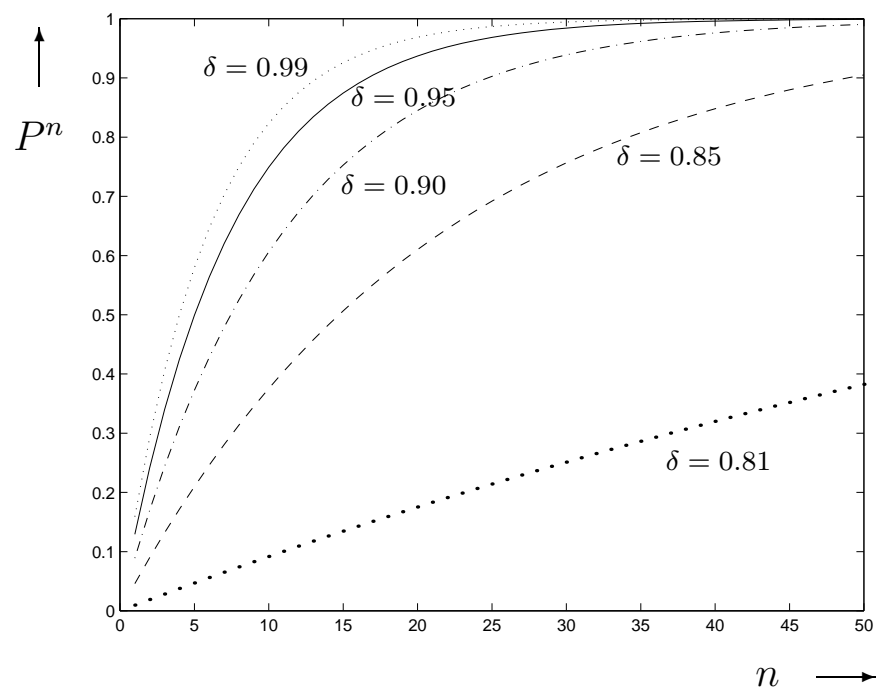

Figure 7.3: Speed of convergence to alternating monopoly for different discount factors.

anything, the alternating monopoly equilibrium is strictly more appealing to both firms, as they each make more profits, irrespective of the initial market structure. In fact, firms are able to extract close to the cartel profits, whereas a cartel is not sustainable as a stationary equilibrium. In the case reported on above, where $\delta=0.95$, the total discounted profits of each firm in the alternating monopoly equilibrium is at least $8.5 \%$ higher than that had in the Cournot equilibrium. This is when both firms are initially in the market. Compare to this end Table 7.1 and Table 7.2. The firm that is first in the market in the initial states with one firm raises its payoff $15.5 \%$ over the Cournot profit. On average, the alternating monopoly equilibrium generates a discounted profit that is almost $12 \%$ higher than Cournot profits. For lower values of $\delta$ this difference becomes smaller, and it eventually converges to zero when $\delta$ gets very close to 0.80 . So, for all values for which the alternating monopoly equilibrium exists, it is strictly payoff dominant.

\subsection{Concluding thoughts}

The previous section reported on the possibility, so far unknown, that in the simplest of dynamic models of entry and exit, where none of the traditional assumptions used to show sustainable dominant positions, such as barriers to entry, simultaneous entry and punishment strategies are made, it is possible - and in fact reasonably likely - that an alternating monopoly arises, with payoffs that approach the cartel outcome, despite the fact that the market allows two firms to make profit when active, and does not sustain a cartel. Consequently also, consumer welfare is importantly harmed by the ability of firms to tacitly collude on this intertemporal market division. Production is decreased below, and prices increased above their duopoly levels, resulting in an 
increase in dead-weight loss. ${ }^{4}$ Hence, antitrust authorities should be wary of this possible tacit collusion - that, on top of being tacit, only unambiguously manifests itself in the long-run.

The simplicity of the model strengthens the surprise of our result. Yet, in some directions it also calls for further inspection. To that end, calculations for a version of the model in which firms do face (re-)entry costs are performed. As these costs are to be incurred time and again in the alternating monopoly equilibrium, they are expected to rule out the equilibrium as a possibility when sufficiently high. It turns out that, with $\delta=0.95$, modest levels of entry costs of almost $8.5 \%$ of the instantaneous monopoly profits still sustain the alternating monopoly equilibrium as a profitable opportunity. ${ }^{5}$ With higher (re-)entry costs the alternating monopoly equilibrium no longer exists, leaving the Cournot equilibrium. This leads to the counterintuitive conclusion that slight barriers to entry can, in fact, support a relatively competitive environment. For all entry costs for which the alternating monopoly equilibrium exists, the latter is strictly payoff dominant.

The introduction of fixed costs, made in every period when producing, does not jeopardize the results either. That is, the alternating monopoly equilibrium exists for various non-specifically chosen parameter values. In fact, as fixed costs are incurred in either type of equilibrium, alternating monopoly or Cournot, they have no specific bearing on the issue at hand. Fixed costs do, however, open possibilities for interesting further research. For example, specifications for which the Cournot equilibrium involves losses, whereas the alternating monopoly equilibrium is still sustainable, are found. This is the case when discount factors and fixed costs are sufficiently high. The reason is that monopolists bear the fixed costs only every second period. The theory of natural monopoly seems, in other words, compatible with firms that serve the market intertemporally.

Finally, of crucial importance to the result does seem to be the number of potential entrants. The two firm model studied here generates a two-periodic cycle within the set of states. Yet, an $n$-firm extension would accordingly stretch this. When the number of firms increases, an alternating monopoly equilibrium might on average imply $n-1$ periods of being inactive for each firm, awaiting its turn to dominate the market every $n$th time. Obviously, this puts the patience of firms to the test, thereby narrowing the window of parameter values for which the alternating monopoly equilibrium exists - in the limit, when the number of firms goes to infinity, eventually closing it. It is, however, very well possible that a different type of equilibrium exists in an $n$-firm version of our model, namely one in which clusters of firms tacitly collude on sharing the market each period with only a restricted number of them. Over time, these clusters then alternate, again increasing profits at the expense of welfare. The existence-question of these conjectured stationary equilibria can be answered by solving the general system of Theorem 7.6.

\footnotetext{
${ }^{4}$ In fact, since in the linear model under consideration dead-weight loss is half of the total profits between the market parties, the discounted welfare loss left in alternating monopoly is also almost $12 \%$ higher on average than that in Cournot equilibrium.

${ }^{5}$ That is, entry costs up to 0.0208 which is $8.33 \%$ of normalized profits, which are equal to 0.25 .
} 


\section{References}

Adams, W.W., And P. Loustaunau (1994), An Introduction to Gröbner Bases, Graduate Studies in Mathematics, Volume 3, American Mathematical Society, Providence.

Allgower, E.L., AND K. Georg (1980), "Simplicial and Continuation Methods for Approximating Fixed Points and Solutions to Systems of Equations," SIAM Review, 22, 28-85.

Allgower, E.L., AND K. Georg (1983), "Predictor-Corrector and Simplicial Methods for Approximating Fixed Points and Zero Points of Nonlinear Mappings," in A. Bachem, M. Grötschel, and B. Korte (eds.), Mathematical Programming: The State of the Art, Springer-Verlag, Berlin, Heidelberg, New York, pp. 15-56.

Allgower, E.L., AND K. Georg (1990), Numerical Continuation Methods: An Introduction, Springer-Verlag, Berlin, Heidelberg.

Allgower, E.L., And K. Georg (1993), “Continuation and Path Following," Acta Numerica, $1-64$.

Audretsch, D.B., W.J. Baumol, And A.E. Burke (2001), "Competition Policy in Dynamic Markets," International Journal of Industrial Organization, 19, 613-634.

Aumann, R.J. (1974), "Subjectivity and Correlation in Randomized Strategies," Journal of Mathematical Economics, 1, 67-96.

Aumann, R.J. (1987), “Correlated Equilibrium as an Expression of Bayesian Rationality," Econometrica, 55, 1-18.

BAin, J. (1949), “A Note on Pricing in Monopoly and Oligopoly," American Economic Review, $39,448-464$.

Baumol, W.J., J.C. PAnzar, And R.D. Willig (1982), Contestable Markets and the Theory of Industry Structure, Harcourt Brace Jovanich, New York.

Bergemann, D., AND J. VÄLIMÄKi (1996), “Learning and Strategic Pricing," Econometrica, 64, 1125-1149.

Bernheim, B.D. (1984), "Strategic Deterence of Sequential Entry into an Industry," The Rand Journal of Economics, 15, 1-11. 
BERnHEIM, D. (1984), “Rationalizable Strategic Behavior,” Econometrica, 52, 1007-1028.

Border, K.C. (1985), Fixed Point Theorems with Applications to Economics and Game Theory, Cambridge University Press, Cambridge.

Borm, P., A. GiJsberts, And S.H. TiJs (1989), "A Geometric-Combinatorial Approach to Bimatrix Games," Methods of Operations Research, 59, 199-209.

Brandenbuger, A., And E. DeKel (1987), "Rationalizability and Correlated Equilibria," Econometrica, 55, 1391-1402.

Breton, M. (1991), “Algorithms for Stochastic Games,” in T.E.S. Raghavan, T.S. Ferguson, T. Parthasarathy, and O.J. Vrieze (eds.), Stochastic Games and Related Topics: In Honor of Professor L.S. Shapley, Theory and Decision Library C: Game Theory, Mathematical Programming and Operations Research: Volume 7, Kluwer Academic Publishers, Dordrecht, pp. 45-57.

Breton, M., J.A. Filar, A. Haurie, And T.A. Schultz (1986), “On the Computation of Equilibria in Discounted Games," in T. Basar (ed.), Dynamic Games and Applications in Economics, Lecture Notes on Economics and Mathematical Systems No. 205, Springer-Verlag, Berlin.

BrouWER, L.E.J. (1912), “Über Abbildung van Mannigfaltigkeiten,” Mathematische Annalen, 71, 97-115.

Browder, F.E. (1960), "On Continuity of Fixed Points under Deformations of Continuous Mappings," Summa Brasiliensis Mathematicae, 4, 183-191.

Brown, D.J., DeMarzo, P.M., And Eaves, B.C. (1996), “Computing Equilibria when Asset Markets are Incomplete," Econometrica, 64, 1-27.

Buchberger, B. (1965), Ein Algorithmus zum Auffinden der Basiselemente des Restklassenringes nach einem nulldimensionalen Polynomideal, Ph.D. Thesis, University of Innsbruck, Innsbruck, Austria.

Chow, S.N., J. Mallet-Paret, And J.A. Yorke (1979), “A Homotopy Method for Locating All Zeros of a System of Polynomials," in H.O. Peitgen, and H.O. Walther (eds.), Functional Differential Equations and Approximation of Fixed Points, 730, Springer-Verlag, Berlin, Heidelberg, New York, pp. 228-237.

Cox, D.A., J.B. LitTle, And D. O’SheA (1996), Ideals, Varieties, and Algorithms: An Introduction to Computational Algebraic Geometry and Commutative Algebra, Undergraduate Texts in Mathematics, Springer-Verlag, New York.

Damme, E.E.C. VAn (1987), Stability and Perfection of Nash Equilibria, Springer-Verlag, New York. 
Damme, E.E.C. VAn (1995), "Game Theory: The Next Stage," in A.P. Kirman, and L.A. Gérard-Varet (eds.), Economics Beyond the Millenium, Oxford University Press, Oxford, pp. 184-214.

Dasgupta, P., And J. Stiglitz (1988), "Potential Competition, Actual Competition, and Economic Welfare," European Economic Review, 32, 569-577.

Debreu, G. (1959), Theory of Value, Yale University Press, New Haven.

Dickhaut, J., And T. Kaplan (1993), “A Program for Finding Nash Equilibria,” in H.R. Varian (ed.), Economic and Financial Modeling with Mathematica, Springer-Verlag, New York.

Dixit, A., AND C. Shapiro (1986), "Entry Dynamics and Mixed Strategies," in L.G. Thomas (ed.), Strategic Planning, Lexington Books, Lexington, Massachusetts, pp. 63-79.

Doup, T.M., G. VAn Der LAAn, AND A.J.J. TAlmAn (1987), “The $\left(2^{(n+1)}-2\right)$-Ray Algorithm: A New Simplicial Algorithm to Compute Economic Equilibria," Mathematical Programming, 39, 241-252.

DreXler, F.J. (1977), "Eine Methode zur Berechnung sämtlicher Lösungen van Polynomgleichungssystemen," Numerisch Mathematik, 29, 45-58.

DreXler, F.J. (1978), "A Homotopy-Method for the Calculation of All Zero-Dimensional Polynomial Ideals," in H.G. Wacker (ed.), Continuation Methods, Academic Press, New York, pp. 69-93.

EAves, B.C. (1971), “The Linear Complementarity Problem,” Management Science, 17, 612634.

EAves, B.C., And K. Schmedders (1999), "General Equilibrium Models and Homotopy Methods," Journal of Economic Dynamics and Control, 23, 1249-1279.

Elzen, A.H. VAn DEN, AND A.J.J. TALMAN (1991), "A Procedure for Finding Nash Equilibria in Bi-Matrix Games," ZOR-Methods and Models of Operation Resarch, 35, 27-43.

Elzen, A.H. VAn DEn, And A.J.J. Talman (1999), "An Algorithmic Approach towards the Tracing Procedure for Bi-matrix Games," Games and Economic Behavior, 28, 130-145.

Filar, J.A., T.A. Schultz, F. ThuiJsman, And O.J. VRieze (1991), "Nonlinear Programming and Stationary Equilibria in Stochastic Games," Mathematical Programming, 50, 227-237. FIlar, J.A., AND O.J. VRIEZE (1997), Competitive Markov Decision Processes, SpringerVerlag, New York.

FINK, A.M. (1964), "Equilibrium in a Stochastic n-Person Game," Journal of Science of Hiroshima University Series A-I, 28, 89-93. 
FudenberG, D., And J. Tirole (1995), Game Theory, MIT Press, Cambridge, Massachusetts. GALILEI, G. (1623), Il Saggiatore (The Assayer), Rome.

Garcia, C.B., C.E. LemKe, AND H.J. Lüthi (1973), "Simplicial Approximation of an Equilibrium Point of Noncooperative N-Person Games," in T.C. Hu, and S.M. Robinson (eds.), Mathematical Programming, Academic Press, New York, pp. 227-260.

Garcia, C.B., AND T.Y. Li (1980), "On the Number of Solutions to Polynomial Systems of Equations," SIAM Journal on Numerical Analysis, 17, 540-546.

Garcia, C.B., AND W.I. Zangwill (1979a), "Finding All Solutions to Polynomial Systems and Other Systems of Equations," Mathematical Programming, 16, 159-176.

Garcia, C.B., AND W.I. Zangwill (1979b), "Determining All Solution to Certain Systems of Nonlinear Equations," Mathematics of Operations Research, 4, 1-14.

Garcia, C.B., AND W.I. Zangwill (1980), “Global Continuation Methods for Finding All Solutions to Polynomial Systems of Equations in $N$ Variables," in A.V. Fiacco, and K.O. Kortanek (eds.), Extremal Methods and Systems Analysis, Springer-Verlag, Berlin, Heidelberg, New York, pp. 481-497.

Garcia, C.B., And W.I. Zangwill (1981), Pathways to Solutions, Fixed Points, and Equilibria, Pentice-Hall Inc., Englewood Cliffs, New Jersey.

Gilbert, R. (1989), “The Role of Potential Competition in Industrial Organization,” Journal of Economic Perspectives, 3, 107-127.

Goldberg, V., and S. Moirao (1973), “Limit Pricing and Potential Competition,” Journal of Political Economics, 81, 1460-1466.

Golubitsky, M., And V. Guillemin (1973), Stable Mappings and Their Singularities, Springer-Verlag, New York.

GRÖBNER, W. (1949), Moderne Algebraische Geometrie, Die Idealtheoretischen Grundlagen, Springer-Verlag, Wien, Innsbruck.

GrÖBNER, W. (1970), Algebraische Geometrie II, Bibliografisches Institut, Mannheim.

Grossman, S.J. (1981), "Nash Equilibrium and the Organization of Markets with Large Fixed Costs," Econometrica, 49, 1149-1172.

Guillemin, V., And A. Pollack (1974), Differential Topology, Prentice-Hall, Inc., Englewood Cliffs, New Jersey.

Haller, H., And R. LagunofF (2000), "Genericity and Markovian Behavior in Stochastic Games," Econometrica, 68, 1231-1248. 
HARSANYI, J.C. (1973), “Oddness of the Number of Equilibrium Points,” International Journal of Game Theory, 2, 235-250.

Harsanyi, J.C. (1975), “The Tracing Procedure: A Bayesian Approach to Defining a Solution for $n$-Person Noncooperative Games," International Journal of Game Theory, 4, 61-94.

HARSANyi, J.C., AND R. SElten (1988), A General Theory of Equilibrium Selection in Games, MIT Press, Cambridge, Massachusetts.

Herings, P.J.J. (1996), Static and Dynamic Aspects of General Disequilibrium Theory, in Theory and Decision Library Series C: Game Theory, Mathematical Programming and Operations Research, Kluwer Academic Publishers, Dordrecht.

Herings, P.J.J. (1997), “A Globally and Universally Stable Price Adjustment Process,” Journal of Mathematical Economics, 27, 163-193.

Herings, P.J.J. (2000), “Two Simple Proofs of the Feasibility of the Linear Tracing Procedure," Economic Theory, 15, 485-490.

Herings, P.J.J., AND A.H. VAn DEn Elzen (2002), "Computation of the Nash Equilibrium Selected by the Tracing Procedure in N-Person Games," Games and Economic Behavior, 38, 89-117.

Herings, P.J.J., And R.J.A.P. PeEters (2000), "Stationary Equilibria in Stochastic Games: Structure, Selection, and Computation," Meteor Research Memorandum 00/031, Universiteit Maastricht, Maastricht.

Herings, P.J.J., And R.J.A.P. Peeters (2001a), “A Differentiable Homotopy to Compute Nash Equilibria of $n$-Person Games," Economic Theory, 18, 159-185.

Herings, P.J.J., And R.J.A.P. PeEters (2001b), "Equilibrium Selection in Stochastic Games,” METEOR Research Memorandum 01/019, Universiteit Maastricht, Maastricht.

Herings, P.J.J., R.J.A.P. Peeters, And M.P. Schinkel (2001), "Intertemporal Market Division: A Case of Alternating Monopoly," METEOR Research Memorandum 01/025, Universiteit Maastricht, Maastricht.

Herings, P.J.J., AND K. Schmedders (2001), "Computing Equilibria in Finance Economies with Incomplete Markets and Transactions Costs," Center for Mathematical Studies in Economics and Management Science, Working Paper 1318, Northwestern University, Evanston, Illinois.

Hildenbrand, W. (1974), Core and Equilibria of a Large Economy, Princeton University Press, Princeton, New Jersey. 
Jongen, H.T., P. JonkeR, And F. Twilt (1983), Nonlinear Optimization in $\mathbb{R}^{n}$, I. Morse Theory, Chebyshev Approximation, Methoden und Verfahren der mathematische Physik, 29, Peter Lang, Frankfurt.

Jongen, H.T., P. Jonker, And F. Twilt (1986), Nonlinear Optimization in $\mathbb{R}^{n}$, II. Transversality, Flows, Parametric Aspects, Methoden und Verfahren der Mathematische Physik, 32, Peter Lang, Frankfurt.

JUDD, K.L. (1997), “Computational Economics and Economic Theory: Substitutes and Complements?," Journal of Economic Dynamics and Control, 21, 907-942.

JudD, K.L. (1998), Numerical Methods in Economics, MIT Press, Cambridge, Massachusetts.

Kakutani, S. (1941), “A Generalization of Brouwer's Fixed Point Theorem,” Duke Mathematical Journal, 8, 457-459.

Kojima, M., And S. Mizuno (1983), "Computation of All Solutions to a System of Polynomial Equations," Mathematical Programming, 25, 131-157.

Kostreva, M.M., And L.A. KinArd (1991), "A Differential Homotopy Approach for Solving Polynomial Optimization Problems and Noncooperative Games," Computers and Mathematics with Applications, 21, 135-143.

LAAN, G. VAN DER, AND A.J.J. TAlman (1982), “On the Computation of Fixed Points in the Product Space of Unit Simplices and an Application to Noncooperative $n$-Person Games,' Mathematics of Operations Research, 7, 1-13.

LAAn, G. VAn Der, A.J.J. TAlman, And L. VAn Der Heyden (1987), “Simplicial Variable Dimension Algorithms for Solving the Nonlinear Complementarity Problem on a Product of Unit Simplices Using a General Labelling," Mathematics of Operation Research, 12, 377-397.

LEMKE, C.E., AND J.T. Howson JR. (1964), "Equilibrium Points of Bimatrix Games," SIAM Journal on Applied Mathematics, 12, 413-423.

Mas-Colell, A. (1974), "A Note on a Theorem of F. Browder," Mathematical Programming, 6, 229-233.

Mas-Colell, A. (1985), The Theory of General Economic Equilibrium: A Differentiable Approach, Cambridge University Press, Cambridge.

Maskin, E., And J. Tirole (2001), "Markov Perfect Equilibrium, I: Observable Actions," Journal of Economic Theory, 100, 191-219.

MCKelvey, R.D. (1996), “A Liapunov Function for Nash Equilibria,” California Institute of Technology, Social Science Working Paper \#953. 
McKelvey, R., And A. MCLennan (1996), "Computation of Equilibria in Finite Games," in H.M. Amman, D.A. Kendrick, and J. Rust (eds.), Handbook of Computational Economics, Volume I, Elsevier Science, Amsterdam, pp. 87-142.

McKelvey, R.D., And A. MCLennan (1997), “The Maximal Number of Regular Totally Mixed Nash Euilibria," Journal of Economic Theory, 72, 411-425.

McLennan, A. (1997), “The Maximal Generic Number of Pure Nash Equilibria,” Journal of Economic Theory, 72, 408-410.

McLennan, A. (1999), “The Expected Number of Nash Equilibria of a Normal Form Game," mimeo.

MilnoR, J.W. (1965), Topology from the Differentiable Viewpoint, The University Press of Virginia, Charlottesville.

Mizuno, S. (1981), "A Simplicial Algorithm for Finding All Solutions to Polynomial Systems of Equations," Research Reports B-97, Department of Information Sciences, Tokyo Institute of Technology, Tokyo.

Modigliani, F. (1958), "New Developments on the Oligopoly Front," Journal of Political Economy, 66, 215-232.

Morgan, A.P. (1983), "A Method for Computing All Solutions to Systems of Polynomial Equations," ACM Transactions on Mathematical Software, 9, 1-17.

Morgan, A.P. (1987), Solving Polynomial Systems Using Continuation for Engineering and Scientific Problems, Prentice-Hall Inc., Upper Saddle River, New Jersey.

Morgan, A.P., And A.J. Sommese (1987), "Computing All Solutions to Polynomial Systems Using Homotopy Continuation," Applied Mathematics and Computation, 24, 115-138.

Morgan, A.P., A.J. Sommese, And L.T. WAtson (1989), "Finding All Isolated Solutions to Polynomial Systems Using HoMPACK," ACM Transactions on Mathematical Software, 15, 93-122.

Myerson, R.B. (1978), "Refinements of the Nash Equilibrium Concept,” International Journal of Game Theory, 7, 73-80.

NASH, J.F. (1950), "Equilibrium Points in N-Person Games," Proceedings of the National Academy of Sciences of the Unites States of America, 36, 48-49.

NASH, J.F. (1951), “Non-Cooperative Games,” Annals of Mathematics, 54, 286-295.

Neumann, J. von (1928), "Zur Theorie der Geselshaftsspiele," Mathematische Annalen, 100, 295-320. 
Neumann, J. von, And O. Morgenstern (1944), Theory of Games and Economic Behavior, Princeton University Press, Princeton.

NTI, K.O. (1989), "More Potential Entrants May Lead to Less Competition,” Journal of Economics, 49, 47-70.

NTI, K.O. (2000), "Potential Competition and Coordination in Market Entry Game," Journal of Economics, 71, 149-165.

Olley, G.S., AND A. PAKes (1996) “The Dynamics of Productivity in the Telecommunications Equipment Industry," Econometrica, 64, 1263-1298.

PAKES, A., AND R. ERICSON (1998), "Empirical Implications of Alternative Models of Firm Dynamics," Journal of Economic Theory, 79, 1-45.

PAKes, A., AND P. MCGuire (2001), "Stochastic Algorithms, Symmetric Markov Perfect Equilibrium, and the 'Curse' of Dimensionality,' Econometrica, 69, 1261-1282.

Parthasarathy, T., And T.E.S. Raghavan (1981), "An Orderfield Property for Stochastic Games when One Player Controls Transition Probabilities,' Journal of Optimization Theory and Applications, 33, 375-392.

PEARCE, D. (1984), "Rationalizable Strategic Behavior and the Problem of Perfection,” Econometrica, 52, 1029-1050.

Rosenmüller, J. (1971), "On a Generalization of the Lemke-Howson Algorithm to Noncooperative N-Person Games," SIAM Journal on Applied Mathematics, 21, 73-79.

Russell, B. (1931), The Scientific Outlook, Allen and Unwin, London.

SCARF, H. (1967), “The Approximation of Fixed Points of a Continuous Mapping," SIAM Journal of Applied Mathematics, 15, 1328-1343.

SCARF, H. (1973), The Computation of Economic Equilibria, Yale University Press, New Haven, Connecticut.

SCHAnUEL, S.H., L.K. Simon, AND W.R. ZAmE (1991), "The Algebraic Geometry of Games and the Tracing Procedure," in R. Selten (ed.), Game Equilibrium Models II: Methods, Morals and Markets, Springer-Verlag, Berlin, pp. 9-43.

Schultz, T.A. (1986), Mathematical Programming and Stochastic Games, Ph.D. Thesis, The John Hopkins University, Baltimore, Maryland.

Selten, R. (1975), "Reexamination of the Perfectness Concept for Equilibrium Points in Extensive Games," International Journal of Game Theory, 4, 25-55.

SElten, R. (1978), “The Chain Store Paradox,” Theory and Decision, 9, 127-159. 
Shapley, L.S. (1953), "Stochastic Games," Proceedings of the National Academy of Sciences of the United States of America, 39, 1095-1100.

Sherman, R., And T. Willett (1967), "Potential Entrants Discourage Entry," Journal of Political Economics, 75, 400-403.

SiER KSMA, G. (2001), "Een Kleine Stap naar het Oneindig Grote," Nieuw Archief voor Wiskunde, 5/2, 210-216.

Sobel, M.J. (1971), “Non-Cooperative Stochastic Games,” The Annals of Mathematical Statistics, 42, 1930-1935.

Stengel, B. von, A.H. van den Elzen, And A.J.J. TAlman (2001), “Computing Normal Form Perfect Equilibria for Extensive Two-Person Games", mimeo. Forthcoming in Econometrica.

Stiglitz, J. (1981), "Potential Competition May Lower Welfare," American Economic Review, 71, 184-189.

Sylos-LABini, P. (1962), Oligopoly and Technical Progress, Harvard University Press, Cambridge, Massachusetts.

TAKahashi, M. (1964), "Equilibrium Points of Stochastic, Noncooperative $n$-Person Games," Journal of Science of Hiroshima University Series A-I, 28, 95-99.

TAlman, A.J.J., And Z. YAng (1998), “Computable Sensible Nash Equilibria,” Yokohama Business Review, 19, 1-15.

Vives, X. (1988), “Sequential Entry, Industry Structure and Welfare,” European Economic Review, 32, 1671-1687.

Waldman, M. (1991), "The Role of Multiple Potential Entrants/Sequential Entry in Noncooperative Entry Deterrence," Rand Journal of Economics, 22, 446-453.

Watson, L.T. (1979), "A Globally Convergent Algorithm for Computing Fixed Points of $C^{2}$ Maps," Applied Mathematics and Computation, 5, 297-311.

Watson, L.T., S.C. Billups, And A.P. Morgan (1987), "HompaCK: A Suite of Codes for Globally Convergent Homotopy Algorithms," ACM Transactions on Mathematical Software, 13, 281-310.

WAtson, L.T., AND D. FEnNER (1980), "Chow-Yorke Algorithm for Fixed Points or Zeros of $C^{2}$ Maps," ACM Transactions on Mathematical Software, 6, 252-260.

WiLson, R. (1971), “Computing Equilibria of n-Person Games,” SIAM Journal of Applied Mathematics, 21, 80-87. 
Wilson, R. (1992), “Computing Simply Stable Equilibria,” Econometrica, 60, 1039-1070.

Wright, A.H. (1985), "Finding All Solutions to a System of Polynomial Equations," Mathematics of Computation, 44, 125-133.

Yamamoto, Y. (1993), "A Path-Following Procedure to Find a Proper Equilibrium of Finite Games," International Journal of Game Theory, 22, 49-59.

Zulehner, W. (1988), "A Simple Homotopy Method for Determining All Isolated Solutions to Polynomial Systems," Mathematics of Computation, 50, 167-177. 


\section{Samenvatting}

Speltheorie houdt zich bezig met het modelleren en oplossen van conflictsituaties. Van oudsher wordt onderscheid gemaakt tussen twee takken binnen de speltheorie: de coöperatieve speltheorie en de niet-coöperatieve speltheorie. De coöperatieve speltheorie bestudeert situaties waarin de betrokken spelers tot bindende afspraken kunnen komen. De niet-coöperatieve speltheorie bestudeert situaties waarin dit niet het geval is. Dit proefschrift richt zich enkel op de tweede tak.

In de niet-coöperatieve speltheorie, die de strategische interactie tussen verschillende spelers bestudeert, gaat de aandacht veelal uit naar het evenwichtsconcept van John Nash. In een Nash evenwichtssituatie gedragen alle spelers zich optimaal, gegeven het gedrag van de overige spelers en gegeven dat het gedrag van alle individuele spelers simultaan uitvoerbaar is. Geen enkele speler zal dus zijn situatie kunnen verbeteren door af te wijken van de actie die hij speelt in de evenwichtssituatie.

In niet-coöperatieve speltheoretische modellen wordt expliciet gemodelleerd dat spelers in hun overwegingen meenemen hoe andere spelers op hun gedrag zullen reageren. In veel van dergelijke modellen zijn meerdere evenwichten mogelijk, die zeer verschillende eigenschappen kunnen hebben. Dit wordt het probleem van multipliciteit van evenwichten genoemd. Ter oplossing van het probleem van multipliciteit zijn er allerlei verfijningen van het Nash evenwichtsconcept geïntroduceerd. Een verfijning legt doorgaans additionele eisen op aan een evenwicht en sluit op deze manier bepaalde evenwichten uit.

De niet-coöperatieve speltheorie kent zowel statische spelen als dynamische spelen. Een statisch spel is een spel dat zich op één tijdsmoment afspeelt: alle spelers maken een keuze en afhankelijk van de gemaakte keuzes vindt een uitbetaling aan iedere speler plaats. In een dynamisch spel moet er op meerdere tijdsmomenten een beslissing genomen worden. Belangrijk in zulke spelen zijn de informatie die een speler heeft over eerder genomen acties, de duur van het spel, en de wijze waarop de uitbetalingen gedefinieerd zijn.

Een succesvolle toepassing van de speltheorie op problemen waarin conflictsituaties voorkomen, wordt vaak belemmerd doordat aanwezige dynamiek de problemen al snel te complex maakt om analytisch (met de hand) te benaderen. Zelfs wanneer er geen dynamische aspecten aanwezig zijn, zullen problemen al snel te complex zijn, wanneer het aantal spelers dan wel het aantal actiemogelijkheden van de spelers toeneemt. Een oplossing hiervoor wordt gevonden 
door nieuwe algoritmen ter berekening van speltheoretische evenwichten te ontwikkelen die expliciet rekening houden met de factor tijd en die goed implementeerbaar zijn op de computer. Bovendien dienen dergelijke algoritmen, in het gebruikelijke geval dat er meerdere evenwichten bestaan, een evenwicht met aantrekkelijke speltheoretische eigenschappen te selecteren.

De eerste klasse van spelen die in dit proefschrift behandeld wordt, is de klasse van statische spelen, de zogenaamde spelen in normale vorm. Voor deze klasse van spelen is een algoritme ontwikkeld dat in staat is een Nash evenwicht te berekenen. Ter oplossing van het probleem van multipliciteit is ervoor gekozen om gebruik te maken van de 'linear tracing procedure'. De linear tracing procedure is geïntroduceerd door John Harsanyi en Reinhard Selten en wordt veelvuldig gebruikt in de door hen geopperde selectietheorie. Het is een wiskundige constructie die subjectieve, a priori verwachtingen transformeert in evenwichtsverwachtingen. Dit proefschrift bewijst dat de linear tracing procedure voor bijna alle spelen in normale vorm goed gedefinieerd is, een resultaat dat weliswaar eerder geformuleerd was, maar waaraan sommige experts twijfelden. Dit resultaat betekent dat het algoritme voor bijna alle spelen een evenwicht kan berekenen.

Voorts wordt er voor deze klasse van spelen een methode beschreven waarmee alle evenwichten berekend kunnen worden. Wederom berust de methode op een aanpassingsproces dat triviale oplossingen via paden verbindt met evenwichts-oplossingen. Het berekenen van alle evenwichten lijkt praktisch onuitvoerbaar door de forse toename van het aantal evenwichten bij toename van het aantal spelers dan wel het aantal actiemogelijkheden per speler. Daarvoor is het algoritme zodanig ontwikkeld dat tijdens zijn uitvoering steeds nieuwe evenwichten gevonden worden. Verder kan de rekentijd aanzienlijk worden verkort door gebruik te maken van parallelle computers.

Dynamische spelen met volledige informatie die van eindige duur zijn en waar spelers hun uitbetalingen ontvangen wanneer alle beslissingen genomen zijn, worden spelen in extensieve vorm genoemd. Voor deze klasse van spelen is het mogelijk het algoritme dat ontworpen is voor de spelen in normale vorm te gebruiken. Dit kan op twee manieren. De eerste manier is door het spel in extensieve vorm te herschrijven als een spel in normale vorm door de verschillende tijdsmomenten waarop een speler een actie moet nemen als afzonderlijke spelers te beschouwen, maar met dezelfde uitbetaling. De tweede manier is om achteraan te beginnen en voor alle deelspelen de evenwichten te bepalen. Met behulp van achterwaartse inductie zal een evenwicht gevonden worden. Dit houdt wel in dat het algoritme herhaaldelijk gebruikt zal moeten worden.

Als laatste type niet-coöperatieve spelen komen de stochastische spelen aan bod, of preciezer, verdisconteerde stochastische spelen met oneindige tijdshorizon. In een stochastisch spel moeten spelers in iedere periode een beslissing nemen. Afhankelijk van deze beslissingen en van het toeval, komt een toestand in de volgende periode tot stand. Deze toestand is van invloed op de mogelijk te nemen beslissingen en de uitbetalingen in die periode. Om dit probleem met oneindige horizon nog analyseerbaar te houden, zullen kansen op toestandsovergangen condi- 
tioneel op de huidige toestand en de in de huidige periode genomen acties, tijdsonafhankelijk verondersteld worden. Dit heeft tot gevolg dat strategieën tijdonafhankelijk (stationair) gekozen kunnen worden. De bedoeling is om zogenaamde stationaire evenwichten te berekenen.

Aangezien we hier wederom met het probleem van multipliciteit te kampen hebben, wordt de selectie aangescherpt. Naast selectie op stationaire evenwichten wordt een uitbreiding op de linear tracing procedure gedefinieerd, de 'stochastic tracing procedure'. Aangezien er meerdere natuurlijke uitbreidingen mogelijk zijn, moet er een weloverwogen keuze gemaakt worden. Gegeven zekere subjectieve a priori stationaire verwachtingen, transformeert de stochastic tracing procedure deze verwachtingen in stationaire evenwichtsverwachtingen. Opnieuw wordt aangetoond dat er een pad gevormd wordt. Een bijkomstig resultaat is dat voor bijna ieder stochastisch spel het aantal stationaire evenwichten oneven is.

Alle onderzochte problemen hebben als overeenkomst dat er paden ontstaan. En wel: stuksgewijs gladde paden, beginnend bij een triviale oplossing en eindigend bij een evenwicht. In alle gevallen is het startpunt van zo'n pad vast te stellen. Indien we een pad vanuit het startpunt volgen komen we uit bij een evenwicht. Voor het volgen van paden zijn verschillende padvolgende methoden voorhanden. In dit proefschrift is voor de implementatie van de algoritmes een padvolgende methode gebruikt die professioneel geprogrammeerd is in FORTRAN. Het betreft hier het softwarepakket genaamd HомРАCK. Voor gebruik van dit pakket is het vereist dat het te volgen pad volledig glad is. Met behulp van een eenvoudige transformatie van variabelen is dit te bereiken. De numerieke prestaties van de aan numeriek onderzoek onderworpen algoritmen zijn uitvoerig beschreven.

Als proef op de som is het algoritme ter berekening van een stationair evenwicht voor stochastische spelen toegepast op een concreet probleem uit de economie. Het betreft een 'market entry-and-exit' model. In zo'n model is er op ieder moment een zeker aantal zittende bedrijven. De zittende bedrijven moeten een beslissing nemen over de hoeveelheid te produceren goederen en of ze het volgende tijdsmoment actief blijven of uittreden. Simultaan moeten potentiële toetreders beslissen of ze toetreden tot de markt of uit de markt blijven. Afhankelijk van de gekozen productiehoeveelheden krijgt ieder zittend bedrijf zijn uitbetaling. Potentiële toetreders betalen toetredingskosten in geval zij er voor kiezen toe te treden. De nieuwe marktsituatie, gegeven door het aantal zittende bedrijven, hangt af van het huidig aantal zittende bedrijven, de beslissingen omtrent uittreding genomen door de zittende bedrijven en de beslissingen omtrent toetreding genomen door de potentiële toetreders. Gegeven de nieuwe marktstructuur, die dan bij alle bedrijven bekend is, worden opnieuw beslissingen genomen. Van dit market entry-and-exit model worden stationaire evenwichten berekend voor verschillende uitbetalingsfuncties. Op basis hiervan is het mogelijk te onderzoeken hoe marktstructuren zich in de loop van de tijd ontwikkelen, alsmede wat de effecten zijn van potentiële concurrentie op prijzen en welvaart.

De situatie met twee bedrijven, afwezigheid van vaste kosten en toetredingskosten is in de- 
tail onderzocht. Indien de vraagcurve linear is en de markt groot genoeg is om door de twee bedrijven bediend te worden, valt het te verwachten dat beide bedrijven permanent op de markt aanwezig zullen zijn. Het herhaalde Cournot evenwicht - resulterende in een situatie waar beide bedrijven altijd productief zijn en blijven - blijkt inderdaad te bestaan. Echter, na toepassing van het ontwikkelde algoritme ter berekening van een stationair evenwicht voor stochastische spelen, blijkt dat er nog een evenwicht bestaat; een evenwicht dat een alternerend monopolie induceert. Op even perioden zal het ene bedrijf de markt dienen, op oneven perioden het andere. Het alternerende monopolie staat bedrijven toe bi-periodiek de volle monopoliewinst te grijpen. Opmerkelijk is dat het alternerende monopolie evenwicht voor beide bedrijven lucratiever is dan het herhaalde Cournot evenwicht. Dit impliceert dat de bedrijven sterke prikkels hebben om op dit evenwicht te coördineren. Dit opmerkelijke resultaat reflecteert nogmaals de potentie van numerieke methoden, namelijk dat ze nieuwe economische inzichten kunnen verschaffen. 


\section{Curriculum Vitae}

Ronald Peeters was born on June 29, 1974 in Beers, The Netherlands. He attended the HAVO at the Merletcollege in Cuijk between 1986 and 1991. The academic year 1991/1992, he studied Mathematics at the Educatieve Faculteit of Hogeschool Gelderland in Nijmegen. Between 1992 and 1997, he studied Mathematics at Katholieke Universiteit Nijmegen. During these studies he worked as a teaching assistent. In 1997 he graduated in Mathematics with specialization in Game Theory. Between 1997 and 1999, Ronald Peeters was Ph.D.-student at the SamenwerkingsOrgaan Brabantse Universiteiten, a cooperation body between Technische Universiteit Eindhoven and Katholieke Universiteit Brabant. In Tilburg he was located at the Department of Econometrics and CentER and in Eindhoven he was located at the Department of Mathematics. Between 1999 and 2001, he continued his Ph.D.-studies at the Department of Economics of Universiteit Maastricht. Since December 2001 Ronald Peeters is assistant professor of economics at Universiteit Maastricht.

Ronald Peeters' research and teaching interests include, apart from game theory and mathematics: microeconomic theory, computational economics, industrial organization, network economics, and the economics of uncertainty and information. 
